\title{
Emergence of Highly Enantioselective Catalytic Activity in a Helical Polymer Mediated by Deracemization of Racemic Pendants
}

\author{
Tomoyuki Ikai, ${ }^{*, \dagger}$ Mitsuka Ando, ${ }^{\dagger}$ Masaki Ito, ${ }^{\dagger}$ Ryoma Ishidate, ${ }^{\dagger}$ Nozomu Suzuki,${ }^{\dagger}$ \\ Katsuhiro Maeda, ${ }^{\S, \|}$ Eiji Yashima*,†,
}

${ }^{\dagger}$ Department of Molecular and Macromolecular Chemistry, Graduate School of Engineering, Nagoya University, Chikusa-ku, Nagoya 464-8603, Japan

${ }^{\ddagger}$ Department of Molecular Design and Engineering, Graduate School of Engineering, Nagoya University, Chikusa-ku, Nagoya 464-8603, Japan

$\S$ Graduate School of Natural Science and Technology, Kanazawa University, Kakuma-machi, Kanazawa 920-1192, Japan

" Nano Life Science Institute (WPI-NanoLSI), Kanazawa University, Kakuma-machi, Kanazawa 920-1192, Japan

E-mail: ikai@chembio.nagoya-u.ac.jp; yashima@chembio.nagoya-u.ac.jp 


\section{Table of Contents}

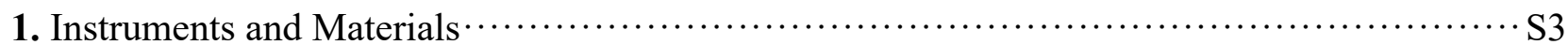

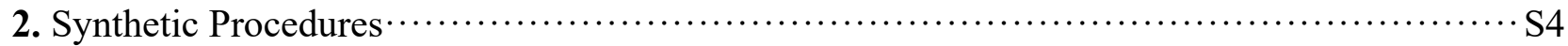

3. Theoretical Studies on the Structures of $(S)-\mathbf{2} \mathbf{a}^{\mathrm{NO}}-\mathrm{OMe}$ and $(S)-\mathbf{2} \mathbf{b}^{\mathrm{NO}}-\mathrm{OMe}$, and Their

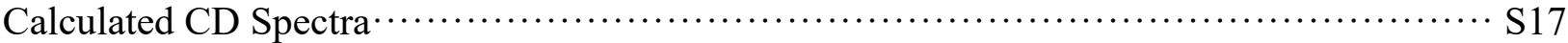

4. Molecular Modeling of One-Handed Helical Cis-Transoidal Poly-(S)-2a ${ }^{\text {NO }}$ and Poly- $(R)-\mathbf{2} \mathbf{b}^{\mathrm{NO}}$

5. Macromolecular Helicity and Axial Chirality Induction in Optically Inactive Poly-rac-2a $\mathbf{a}^{\text {NO }}$ with Enantiomeric Alcohols and Subsequent Dual Static Memory Mediated by Deracemization of the Axially Chiral Biaryl Pendants

6. Asymmetric Allylation of Benzaldehyde with Allyltrichlorosilane Catalyzed by Helical Polymers

7. Supporting Data

8. Supporting References

9. ${ }^{1} \mathrm{H}$ and ${ }^{13} \mathrm{C}$ NMR Spectral Data 


\section{Instruments and Materials}

Instruments. The melting points were measured on a Yanako melting point apparatus (Yanako, Kyoto, Japan) and were uncorrected. The NMR spectra were measured using a Varian 500AS (Agilent Technologies, Santa Clara, CA) or a Bruker Ascend 500 (Bruker Biospin, Billerica, MA) spectrometer operating at $500 \mathrm{MHz}$ for ${ }^{1} \mathrm{H}$ and $126 \mathrm{MHz}$ for ${ }^{13} \mathrm{C}$ using tetramethylsilane as the internal standard. The IR spectra were recorded on a JASCO FT/IR-680 spectrophotometer (JASCO, Tokyo, Japan). The Raman spectra were measured on a confocal Raman microscope (Renishaw inVia Raman system, Renishaw, Inc., Gloucestershire, UK) with a 532-nm excitation laser. The absorption and circular dichroism (CD) spectra were obtained in a 1.0-mm quartz cell using a JASCO V-750 spectrophotometer and a JASCO J-820 spectropolarimeter, respectively. The concentrations of the polymers were calculated based on the monomer units. The temperature was controlled with JASCO ETCS-900 and JASCO PLC-423L apparatuses for absorption and CD measurements, respectively. The size exclusion chromatography (SEC) measurements were performed with a JASCO PU-4580 liquid chromatograph equipped with a JASCO CO-4060 column oven and a JASCO MD-2018 multiwavelength UV/VIS detector. The number-average molar mass $\left(M_{\mathrm{n}}\right)$ and its distribution $\left(M_{\mathrm{w}} / M_{\mathrm{n}}\right)$ were determined at $40{ }^{\circ} \mathrm{C}$ using a Tosoh TSKgel Multipore $\mathrm{H}_{\mathrm{XL}}-\mathrm{M}(30 \mathrm{~cm} \times 7.8 \mathrm{~cm}$ (i.d.)) SEC column (Tosoh, Tokyo, Japan), and tetrahydrofuran (THF) containing $0.5 \mathrm{wt} \%$ tetrabutylammonium bromide (TBAB) was used as the eluent at a flow rate of $1.0 \mathrm{~mL} / \mathrm{min}$. The molar mass calibration curve was obtained with polystyrene standards (Tosoh). Recycling preparative high-performance liquid chromatography (HPLC) was performed with a JAI LC-7080 liquid chromatograph (JAI, Tokyo, Japan) equipped with a JAI UV-800LA UV detector at room temperature. JAIGEL-1HR and JAIGEL-2HR $(60 \mathrm{~cm} \times 2.0 \mathrm{~cm}$ (i.d. $))$ connected in series were used as columns (JAI), and chloroform was used as the eluent at a flow rate of $9.0 \mathrm{~mL} / \mathrm{min}$. The chromatographic separations of enantiomers were performed using HPLC equipped with a JASCO PU-4185 Binary HPLC pump, a JASCO AS4050 autosampler, JASCO MD-2018 multi-wavelength UV/VIS and JASCO CD-4095 dual detectors, and a CHIRALCEL OD-H or a CHIRALPAK IB (DAICEL, Osaka, Japan) was used as a chiral column. The high-resolution mass spectra (HRMS) were recorded on a JEOL JMS-T100CS spectrometer with electrospray ionization (ESI) (JEOL, Tokyo, Japan). Elemental analyses were performed by the laboratory of elemental analyses in the Department of Agriculture, Nagoya University.

Materials. All starting materials and anhydrous solvents were purchased from Sigma-Aldrich (St. Louis, MO), Wako Pure Chemical Industries (Osaka, Japan), Tokyo Kasei (TCI, Tokyo, Japan), Nacalai Tesque (Kyoto, Japan), or Kanto Kagaku (Tokyo, Japan) and were used as received, except for triethylamine $\left(\mathrm{Et}_{3} \mathrm{~N}\right)$ and $\mathrm{THF}$. $\mathrm{NEt}_{3}$ was dried over $\mathrm{KOH}$ pellets and distilled onto $\mathrm{KOH}$ under 
nitrogen. THF was dried and deoxygenized by passing through purification columns (Glass Contour Solvent System, Nikko Hansen, Osaka, Japan), further dried over $\mathrm{LiAlH}_{4}$ under nitrogen, and distilled under high vacuum just before use. Butyl 4-bromo-3-methoxymethoxybenzoate was synthesized according to the previously reported method. ${ }^{\mathrm{S} 1}$

\section{Synthetic Procedures}

Monomers rac-2 $\mathbf{a}^{\mathbf{N O}}$ and $r a c-2 \mathbf{b}^{\mathbf{N O}}$ were prepared according to Schemes S1 and S2, respectively.

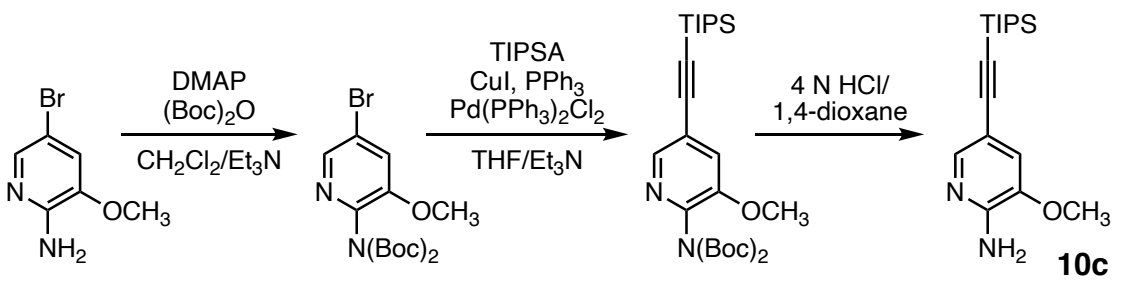

$10 a$

10b

$$
\downarrow \begin{gathered}
\mathrm{CuBr}_{2} \\
\text { amyl nitrite } \\
\mathrm{CH}_{2} \mathrm{Br}_{2}
\end{gathered}
$$

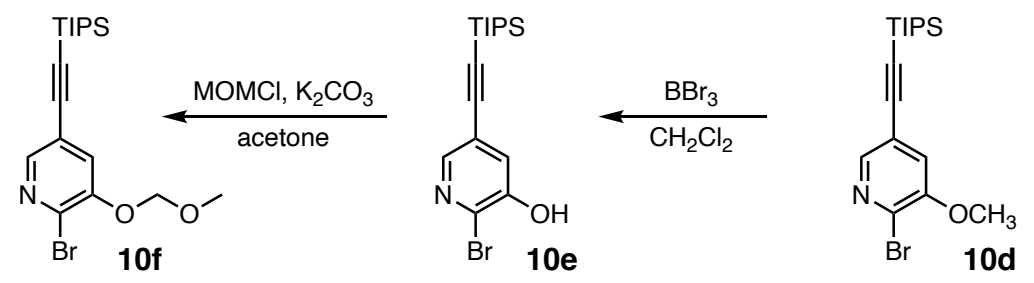
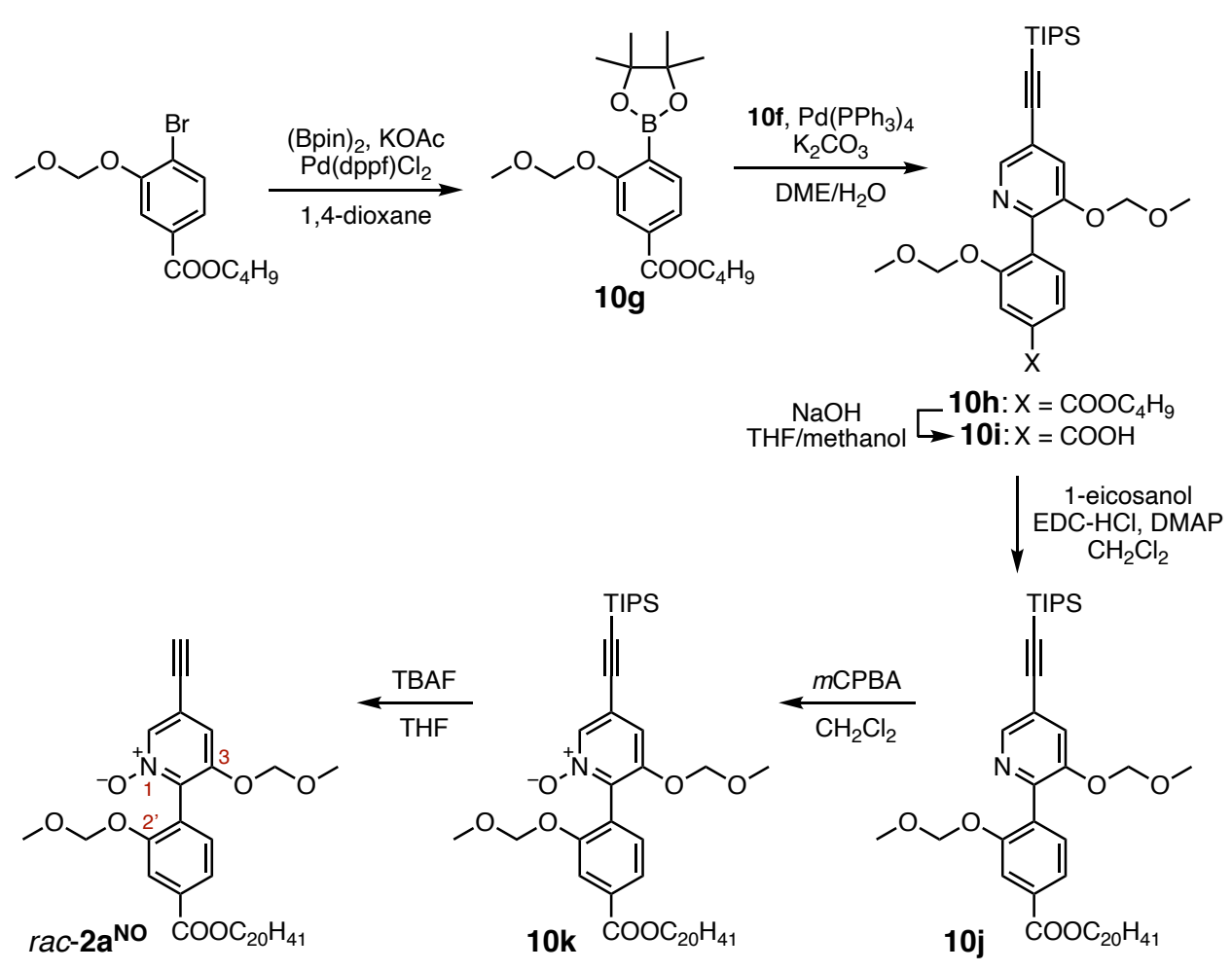

Scheme S1. Synthesis of $r a c-2 a^{\text {NO }}$. 
Synthesis of 5-bromo-2-(bis(tert-butoxycarbonyl)amino)-3-methoxypyridine (10a). To a

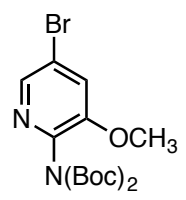

10a mixture of 2-amino-5-bromo-3-methoxypyridine (4.00 g, $19.7 \mathrm{mmol}), N, N$-dimethyl4-aminopyridine (DMAP) (0.11 g, $0.89 \mathrm{mmol})$, and di-tert-butyl dicarbonate $\left((\mathrm{Boc})_{2} \mathrm{O}\right)(10.8 \mathrm{~g}, 49.4 \mathrm{mmol})$ in anhydrous dichloromethane $(100 \mathrm{~mL})$ was added $\mathrm{Et}_{3} \mathrm{~N}(4.2 \mathrm{~mL}, 30 \mathrm{mmol})$. The mixture was stirred at room temperature for $24 \mathrm{~h}$. After evaporating the solvent, the residue was diluted with chloroform and the solution was washed with aqueous $1 \mathrm{~N} \mathrm{HCl}$ and brine, and then dried over $\mathrm{Na}_{2} \mathrm{SO}_{4}$. The solvent was removed under reduced pressure and the crude product was purified by silica gel chromatography using chloroform as the eluent to give the desired product as a white solid (7.04 g, 89\% yield). Mp: $77.6-78.3{ }^{\circ} \mathrm{C}$. IR ( $\mathrm{KBr}$, $\left.\mathrm{cm}^{-1}\right): 1754(\mathrm{C}=\mathrm{O}) .{ }^{1} \mathrm{H}$ NMR $\left(500 \mathrm{MHz}, \mathrm{CDCl}_{3}, 25^{\circ} \mathrm{C}\right): \delta 8.15(\mathrm{~d}, J=1.5 \mathrm{~Hz}, 1 \mathrm{H}, \mathrm{Ar}-\mathrm{H}), 7.37(\mathrm{~d}, J$ $=1.5 \mathrm{~Hz}, 1 \mathrm{H}, \mathrm{Ar}-\mathrm{H}), 3.86\left(\mathrm{~s}, 3 \mathrm{H}, \mathrm{OCH}_{3}\right), 1.41\left(\mathrm{~s}, 18 \mathrm{H}, \mathrm{CH}_{3}\right) \cdot{ }^{13} \mathrm{C} \mathrm{NMR}\left(126 \mathrm{MHz}, \mathrm{CDCl}_{3}, 25{ }^{\circ} \mathrm{C}\right)$ : $\delta 151.10,150.54,141.26,140.48,122.08,119.79,82.95,55.93,27.87$. HRMS (ESI +$): m / z$ calcd for $\mathrm{C}_{16} \mathrm{H}_{23} \mathrm{BrN}_{2} \mathrm{NaO}_{5}\left(\mathrm{M}+\mathrm{Na}^{+}\right), 425.0683$; found 425.0687 .

\section{Synthesis}

of 2-(bis(tert-butoxycarbonyl)amino)-3-methoxy-5((triisopropylsilyl)ethynyl)pyridine (10b). To a mixture of $\mathbf{1 0 a}(7.00 \mathrm{~g}, 17.4 \mathrm{mmol})$,

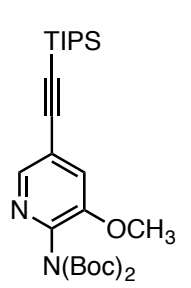

$10 \mathrm{~b}$ triphenylphosphine (0.18 g, $0.69 \mathrm{mmol})$, and bis(triphenylphosphine)palladium(II) dichloride $\left(\mathrm{Pd}\left(\mathrm{PPh}_{3}\right)_{2} \mathrm{Cl}_{2}\right)(0.12 \mathrm{~g}, 0.17 \mathrm{mmol})$ in anhydrous THF/Et $3 \mathrm{~N}(3 / 1, \mathrm{v} / \mathrm{v} ; 68$ $\mathrm{mL})$ was added (triisopropylsilyl)acetylene (TIPSA) $(4.0 \mathrm{~mL}, 18 \mathrm{mmol})$ and copper(I) iodide (CuI) $(66 \mathrm{mg}, 0.35 \mathrm{mmol})$. The mixture was stirred at $60{ }^{\circ} \mathrm{C}$ for 5 h. After evaporating the solvent, the residue was diluted with ethyl acetate and the solution was washed with water and brine, and then dried over $\mathrm{Na}_{2} \mathrm{SO}_{4}$. The solvent was removed under reduced pressure and the crude product was purified by silica gel chromatography using $n$-hexane/ethyl acetate $(8 / 1, \mathrm{v} / \mathrm{v})$ as the eluent to give the desired product as a yellow oil $(8.76$ g, 99\% yield). IR ( $\left.\mathrm{KBr}, \mathrm{cm}^{-1}\right): 2155(\mathrm{C} \equiv \mathrm{C}), 1757(\mathrm{C}=\mathrm{O}) .{ }^{1} \mathrm{H}$ NMR $\left(500 \mathrm{MHz}, \mathrm{CDCl}_{3}, 25{ }^{\circ} \mathrm{C}\right): \delta 8.16$ $(\mathrm{d}, J=1.8 \mathrm{~Hz}, 1 \mathrm{H}, \mathrm{Ar}-\mathrm{H}), 7.23(\mathrm{~d}, J=1.8 \mathrm{~Hz}, 1 \mathrm{H}, \mathrm{Ar}-\mathrm{H}), 3.86\left(\mathrm{~s}, 3 \mathrm{H}, \mathrm{OCH}_{3}\right), 1.40\left(\mathrm{~s}, 18 \mathrm{H}, \mathrm{CH}_{3}\right)$, 1.14-1.15 (m, 21H, TIPS). ${ }^{13} \mathrm{C}$ NMR (126 MHz, $\left.\mathrm{CDCl}_{3}, 25{ }^{\circ} \mathrm{C}\right): \delta 150.61,150.01,143.00,141.82$, 121.45, 120.91, 103.04, 95.03, 82.75, 55.67, 27.89, 18.65, 11.26. HRMS (ESI+): $\mathrm{m} / z$ calcd for $\mathrm{C}_{27} \mathrm{H}_{44} \mathrm{~N}_{2} \mathrm{NaO}_{5} \mathrm{Si}\left(\mathrm{M}+\mathrm{Na}^{+}\right)$, 527.2912; found 527.2920. 
Synthesis of 2-amino-3-methoxy-5-((triisopropylsilyl)ethynyl)pyridine (10c). 10b (8.70 g,

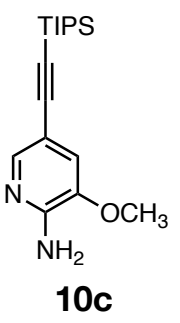

$17.2 \mathrm{mmol}$ ) was dissolved in $4 \mathrm{~N} \mathrm{HCl}$ in 1,4-dioxane $(300 \mathrm{~mL})$ and the solution was stirred at room temperature for $3 \mathrm{~h}$. After evaporating the solvent, the residue was diluted with chloroform and the solution was washed with saturated aqueous $\mathrm{NaHCO}_{3}$, and then dried over $\mathrm{Na}_{2} \mathrm{SO}_{4}$. After removing the solvent by evaporation, the crude $2 \mathrm{c}$ (5.24 g) was obtained as a pale yellow solid and was used for the next step without further purification.

Synthesis of 2-bromo-3-methoxy-5-((triisopropylsilyl)ethynyl)pyridine (10d). To a mixture of

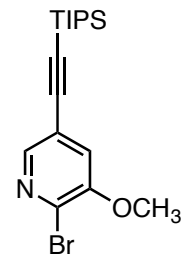

10d the crude 10c (5.20 g) and copper(II) bromide $\left(\mathrm{CuBr}_{2}\right)(1.91 \mathrm{~g}, 8.55 \mathrm{mmol})$ in dibromomethane $(102 \mathrm{~mL})$ was slowly added amyl nitrite $(2.7 \mathrm{~mL}, 20 \mathrm{mmol})$. The mixture was stirred at room temperature for $16 \mathrm{~h}$. After the addition of aqueous $\mathrm{NaOH}$ $(1.0 \mathrm{M}, 10 \mathrm{~mL})$ to quench the reaction, the mixture was diluted with chloroform and the solution was washed with water and brine, and then dried over $\mathrm{Na}_{2} \mathrm{SO}_{4}$. The solvent was removed under reduced pressure and the crude product was purified by silica gel chromatography using $n$-hexane/ethyl acetate $(20 / 1, \mathrm{v} / \mathrm{v})$ as the eluent to give the desired product as a pale yellow oil (3.11 g, 49\% yield over 2 steps). IR ( $\left.\mathrm{KBr}, \mathrm{cm}^{-1}\right)$ : $2158(\mathrm{C} \equiv \mathrm{C}) .{ }^{1} \mathrm{H}$ NMR $\left(500 \mathrm{MHz}, \mathrm{CDCl}_{3}, 25{ }^{\circ} \mathrm{C}\right)$ : $\delta 8.06(\mathrm{~d}, J=1.8 \mathrm{~Hz}, 1 \mathrm{H}, \mathrm{Ar}-\mathrm{H}), 7.13(\mathrm{~d}, J=1.8 \mathrm{~Hz}, 1 \mathrm{H}, \mathrm{Ar}-\mathrm{H}), 3.93\left(\mathrm{~s}, 3 \mathrm{H}, \mathrm{OCH}_{3}\right), 1.13-1.15(\mathrm{~m}$, 21H, TIPS). $\left.{ }^{13} \mathrm{C} \mathrm{NMR} \mathrm{(126} \mathrm{MHz,} \mathrm{CDCl}_{3}, 25{ }^{\circ} \mathrm{C}\right): \delta 152.22,144.08,132.18,120.80,120.51,102.21$, 96.05, 56.30, 18.63, 11.22. HRMS (ESI+): $m / z$ calcd for $\mathrm{C}_{17} \mathrm{H}_{27} \mathrm{BrNOSi}\left(\mathrm{M}+\mathrm{H}^{+}\right), 368.1040$; found 368.1030 .

Synthesis of 2-bromo-3-hydroxy-5-((triisopropylsilyl)ethynyl)pyridine (10e). To a solution of

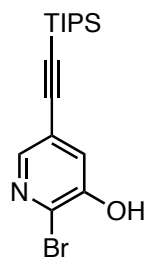

$10 \mathrm{e}$ $\operatorname{10d}(1.62 \mathrm{~g}, 4.40 \mathrm{mmol})$ in anhydrous dichloromethane $(22 \mathrm{~mL})$ was slowly added boron tribromide (1.0 $\mathrm{M}$ in dichloromethane, $18 \mathrm{~mL}, 18 \mathrm{mmol})$. The mixture was stirred at room temperature for $24 \mathrm{~h}$. After quenching the reaction with water and evaporating the solvent, the residue was diluted with ethyl acetate and the solution was washed with water and brine, and then dried over $\mathrm{Na}_{2} \mathrm{SO}_{4}$. The solvent was removed under reduced pressure and the crude product was purified by silica gel chromatography using $n$-hexane/ethyl acetate $(4 / 1, \mathrm{v} / \mathrm{v})$ as the eluent to give the desired product as a white solid (1.37 $\mathrm{g}, 88 \%$ yield). Mp: 132.1-133.0 ${ }^{\circ} \mathrm{C}$. IR ( $\left.\mathrm{KBr}, \mathrm{cm}^{-1}\right): 2147(\mathrm{C} \equiv \mathrm{C}) .{ }^{1} \mathrm{H}$ NMR $\left(500 \mathrm{MHz}, \mathrm{CDCl}_{3}, 25^{\circ} \mathrm{C}\right): \delta 8.04(\mathrm{~d}, J=1.8$ $\mathrm{Hz}, 1 \mathrm{H}, \mathrm{Ar}-\mathrm{H}), 7.34$ (d, $J=2.1 \mathrm{~Hz}, 1 \mathrm{H}, \mathrm{Ar}-\mathrm{H}), 5.54$ (s, 1H, OH), 1.11-1.13 (m, 21H, TIPS). ${ }^{13} \mathrm{C}$ NMR $\left(126 \mathrm{MHz}, \mathrm{CDCl}_{3}, 25^{\circ} \mathrm{C}\right): \delta 148.93,144.49,130.25,125.65,121.50,101.71,96.39,18.60$, 11.18. HRMS (ESI+): $m / z$ calcd for $\mathrm{C}_{16} \mathrm{H}_{25} \operatorname{BrNOSi}\left(\mathrm{M}+\mathrm{H}^{+}\right)$, 354.0883; found 354.0896. 
Synthesis of 2-bromo-3-methoxymethoxy-5-((triisopropylsilyl)ethynyl)pyridine (10f). To a

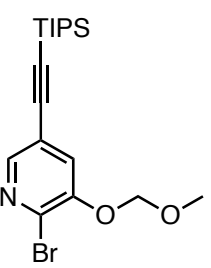

$10 f$

mixture of $10 \mathrm{e}(1.37 \mathrm{~g}, 3.87 \mathrm{mmol})$ and potassium carbonate $(1.23 \mathrm{~g}, 8.90 \mathrm{mmol})$ in anhydrous acetone $(14 \mathrm{~mL})$ was added chloromethyl methyl ether $(0.32 \mathrm{~mL}, 4.2$ $\mathrm{mmol}$ ). The mixture was stirred at $70{ }^{\circ} \mathrm{C}$ for $5 \mathrm{~h}$. After evaporating the solvent, the residue was diluted with ethyl acetate and the solution was washed with water and brine, and then dried over $\mathrm{Na}_{2} \mathrm{SO}_{4}$. The solvent was removed under reduced pressure and the crude product was purified by silica gel chromatography using $n$-hexane/ethyl acetate (15/1, $\mathrm{v} / \mathrm{v})$ as the eluent to give the desired product as a white solid (1.16 g, 75\% yield). Mp: $56.5-57.1{ }^{\circ} \mathrm{C}$. IR $\left(\mathrm{KBr}, \mathrm{cm}^{-1}\right): 2154(\mathrm{C} \equiv \mathrm{C}) .{ }^{1} \mathrm{H}$ NMR $\left(500 \mathrm{MHz}, \mathrm{CDCl}_{3}, 25^{\circ} \mathrm{C}\right): \delta 8.11(\mathrm{~d}, J=1.8 \mathrm{~Hz}, 1 \mathrm{H}, \mathrm{Ar}-\mathrm{H})$, $7.41(\mathrm{~d}, J=2.1 \mathrm{~Hz}, 1 \mathrm{H}, \mathrm{Ar}-\mathrm{H}), 5.28\left(\mathrm{~s}, 2 \mathrm{H}, \mathrm{OCH}_{2} \mathrm{O}\right), 3.54$ (s, 3H, $\left.\mathrm{OCH}_{3}\right), 1.12-1.15$ (m, 21H, TIPS). ${ }^{13} \mathrm{C} \mathrm{NMR}\left(126 \mathrm{MHz}, \mathrm{CDCl}_{3}, 25{ }^{\circ} \mathrm{C}\right): \delta 150.10,145.49,132.91,124.91,120.56,102.02,96.13,95.05$, 56.73, 18.63, 11.22. HRMS (ESI+): $m / z$ calcd for $\mathrm{C}_{18} \mathrm{H}_{29} \mathrm{BrNO}_{2} \mathrm{Si}\left(\mathrm{M}+\mathrm{Na}^{+}\right), 398.1145$; found 398.1169.

Synthesis of 2-methoxymethoxy-4-(butoxycarbonyl)phenylboronic acid pinacol ester (10g).

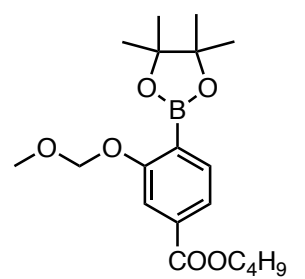

$10 \mathrm{~g}$

To a mixture of butyl 4-bromo-3-methoxymethoxybenzoate (1.52 g, $4.79 \mathrm{mmol})$, potassium acetate $(1.90 \mathrm{~g}, 19.3 \mathrm{mmol})$, and bis(pinacolato)diboron (1.44 g, 5.66 $\mathrm{mmol})$ in anhydrous 1,4-dioxane $(8 \mathrm{~mL})$ was added [1,1'bis(diphenylphosphino)ferrocene]palladium(II) dichloride $\left(\mathrm{Pd}(\mathrm{dppf}) \mathrm{Cl}_{2}\right)(0.20 \mathrm{~g}$, $0.27 \mathrm{mmol}$ ). The mixture was stirred at $100{ }^{\circ} \mathrm{C}$ for $20 \mathrm{~h}$. After evaporating the solvent, the residue was passed through a short pad of silica gel using $n$-hexane/ethyl acetate (5/1, $\mathrm{v} / \mathrm{v})$ as the eluent. After removing the solvent by evaporation, the crude $10 \mathrm{~g}(2.24 \mathrm{~g})$ was obtained as a yellow oil and was used for the next step without further purification.

Synthesis of 10h. To a mixture of $10 f(0.83 \mathrm{~g}, 2.3 \mathrm{mmol})$, the crude $10 \mathrm{~g}(0.71 \mathrm{~g})$, and potassium<smiles>COCOc1cc(C(=O)O[Na])ccc1-c1ncc(C#CC(F)(F)F)cc1OCOC</smiles>
carbonate $(0.73 \mathrm{~g}, 5.3 \mathrm{mmol})$ in a degassed 1,2-dimethoxyethane (DME)/water mixture $(3 / 1, \mathrm{v} / \mathrm{v} ; 60 \mathrm{~mL})$ was added tetrakis(triphenylphosphine)palladium(0) $\left(\mathrm{Pd}\left(\mathrm{PPh}_{3}\right)_{4}\right)(0.20 \mathrm{~g}, 0.18 \mathrm{mmol})$. The mixture was stirred at $80{ }^{\circ} \mathrm{C}$ for $5 \mathrm{~h}$. After evaporating the solvent, the residue was diluted with ethyl acetate and the solution was washed with water and brine, and then dried over $\mathrm{Na}_{2} \mathrm{SO}_{4}$. The solvent was removed under reduced pressure and the crude product was purified by silica gel chromatography using $n$-hexane/ethyl acetate $(3 / 1, \mathrm{v} / \mathrm{v})$ as the eluent to give the desired product as a pale yellow oil $\left(0.89 \mathrm{~g}, 90 \%\right.$ yield). IR $\left(\mathrm{KBr}, \mathrm{cm}^{-1}\right): 2147(\mathrm{C} \equiv \mathrm{C}), 1719(\mathrm{C}=\mathrm{O})$. ${ }^{1} \mathrm{H}$ NMR $\left(500 \mathrm{MHz}, \mathrm{CDCl}_{3}, 25^{\circ} \mathrm{C}\right): \delta 8.44(\mathrm{~d}, J=1.8 \mathrm{~Hz}, 1 \mathrm{H}, \mathrm{Ar}-\mathrm{H}), 7.87(\mathrm{~d}, J=1.5 \mathrm{~Hz}, 1 \mathrm{H}, \mathrm{Ar}-$ H), 7.79 (dd, $J=7.9,1.5 \mathrm{~Hz}, 1 \mathrm{H}, \mathrm{Ar}-\mathrm{H}), 7.58$ (d, $J=1.8 \mathrm{~Hz}, 1 \mathrm{H}, \mathrm{Ar}-\mathrm{H}), 7.39$ (d, $J=7.9 \mathrm{~Hz}, 1 \mathrm{H}$, 
Ar-H), $5.13\left(\mathrm{~s}, 2 \mathrm{H}, \mathrm{OCH}_{2} \mathrm{O}\right), 5.12\left(\mathrm{~s}, 2 \mathrm{H}, \mathrm{OCH}_{2} \mathrm{O}\right), 4.34\left(\mathrm{t}, \mathrm{J}=6.8 \mathrm{~Hz}, 2 \mathrm{H}, \mathrm{OCH}_{2} \mathrm{CH}_{2}\right), 3.38(\mathrm{~s}, 3 \mathrm{H}$, $\mathrm{OCH}_{3}$ ), 3.35 (s, $3 \mathrm{H}, \mathrm{OCH}_{3}$ ), 1.77 (quint, $\left.J=8.0 \mathrm{~Hz}, 2 \mathrm{H}, \mathrm{OCH}_{2} \mathrm{CH}_{2}\right), 1.45-1.52\left(\mathrm{~m}, 2 \mathrm{H}, \mathrm{CH}_{2} \mathrm{CH}_{3}\right.$ ), 1.14-1.17 (m, 21H, TIPS), 0.99 (t, $\left.J=7.4 \mathrm{~Hz}, 3 \mathrm{H}, \mathrm{CH}_{2} \mathrm{CH}_{3}\right) \cdot{ }^{13} \mathrm{C} \mathrm{NMR}\left(126 \mathrm{MHz}, \mathrm{CDCl}_{3}, 25{ }^{\circ} \mathrm{C}\right): \delta$ $166.30,154.99$, 151.13, 147.02, 145.75, 132.74, 132.00, 130.73, 124.72, 123.13, 120.50, 116.02, 103.29, 95.13, 95.07, 94.96, 65.04, 56.24, 56.10, 30.77, 19.28, 18.67, 13.80, 11.27. HRMS (ESI+): $m / z$ calcd for $\mathrm{C}_{31} \mathrm{H}_{45} \mathrm{NNaO}_{6} \mathrm{Si}\left(\mathrm{M}+\mathrm{Na}^{+}\right)$, 578.2908; found 578.2911.

Synthesis of 10i. To a solution of $\mathbf{1 0 h}(0.89 \mathrm{~g}, 1.6 \mathrm{mmol})$ in a THF/methanol mixture $(2 / 1, \mathrm{v} / \mathrm{v}$;

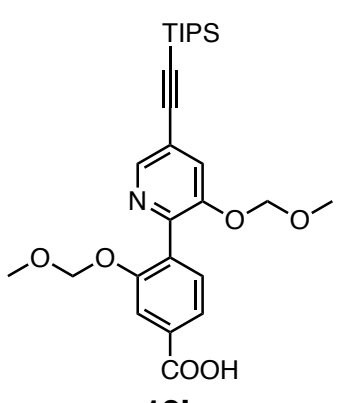

$10 i$ $0.60 \mathrm{~mL})$ was added $\mathrm{NaOH}(96 \mathrm{mg}, 2.4 \mathrm{mmol})$ at $0{ }^{\circ} \mathrm{C}$. The mixture was stirred at room temperature for $12 \mathrm{~h}$. After evaporating the solvent, the residue was diluted with diethyl ether and the solution was washed with aqueous $1 \mathrm{~N} \mathrm{HCl}$ and water, and then dried over $\mathrm{Na}_{2} \mathrm{SO}_{4}$. After removing the solvent by evaporation, the crude $10 \mathbf{i}(0.76 \mathrm{~g})$ was obtained as a white solid and was used for the next step without further purification.

Synthesis of 10j. To a mixture of the crude 10i $(0.76 \mathrm{~g})$, DMAP $(0.22 \mathrm{~g}, 1.8 \mathrm{mmol})$, and 1-<smiles>COCOc1cc(C(=O)OC)ccc1-c1ncc(C#CC(=O)OC)cc1OCOC</smileseicosanol $(0.53 \mathrm{~g}, 1.8 \mathrm{mmol})$ in anhydrous dichloromethane $(6 \mathrm{~mL})$ was added 1-ethyl-3-(3-dimethylaminopropyl)carbodiimide hydrochloride (EDC-HCl) $(0.35 \mathrm{~g}, 1.8 \mathrm{mmol})$ at $0{ }^{\circ} \mathrm{C}$. The mixture was stirred at room temperature for $12 \mathrm{~h}$. After evaporating the solvent, the residue was diluted with chloroform and the solution was washed with aqueous $1 \mathrm{~N} \mathrm{HCl}$, aqueous $1 \mathrm{~N} \mathrm{NaOH}$, and brine, and then dried over $\mathrm{Na}_{2} \mathrm{SO}_{4}$. The solvent was removed under reduced pressure and the crude product was purified by silica gel chromatography using $n$-hexane/ethyl acetate $(8 / 1, \mathrm{v} / \mathrm{v})$ as the eluent to give the desired product as a white solid $(0.90 \mathrm{~g}, 76 \%$ yield over 2 steps). Mp: $38.0-38.8^{\circ} \mathrm{C}$. IR ( $\left.\mathrm{KBr}, \mathrm{cm}^{-1}\right): 2155(\mathrm{C} \equiv \mathrm{C}), 1717(\mathrm{C}=\mathrm{O}) .{ }^{1} \mathrm{H}$ NMR $\left(500 \mathrm{MHz}, \mathrm{CDCl}_{3}\right.$, $\left.25^{\circ} \mathrm{C}\right): \delta 8.44(\mathrm{~d}, J=1.5 \mathrm{~Hz}, 1 \mathrm{H}, \mathrm{Ar}-\mathrm{H}), 7.86(\mathrm{~d}, J=1.5 \mathrm{~Hz}, 1 \mathrm{H}, \mathrm{Ar}-\mathrm{H}), 7.78(\mathrm{dd}, J=7.6,1.5 \mathrm{~Hz}$, $1 \mathrm{H}, \mathrm{Ar}-\mathrm{H}), 7.58(\mathrm{~d}, J=1.8 \mathrm{~Hz}, 1 \mathrm{H}, \mathrm{Ar}-\mathrm{H}), 7.38$ (d, $J=7.6 \mathrm{~Hz}, 1 \mathrm{H}, \mathrm{Ar}-\mathrm{H}), 5.13\left(\mathrm{~s}, 2 \mathrm{H}, \mathrm{OCH}_{2} \mathrm{O}\right)$, $5.11\left(\mathrm{~s}, 2 \mathrm{H}, \mathrm{OCH}_{2} \mathrm{O}\right), 4.32\left(\mathrm{t}, J=6.8 \mathrm{~Hz}, 2 \mathrm{H}, \mathrm{OCH}_{2} \mathrm{CH}_{2}\right), 3.38\left(\mathrm{~s}, 3 \mathrm{H}, \mathrm{OCH}_{3}\right), 3.35\left(\mathrm{~s}, 3 \mathrm{H}, \mathrm{OCH}_{3}\right)$, 1.77 (quint, $J=7.5 \mathrm{~Hz}, 2 \mathrm{H}, \mathrm{OCH}_{2} \mathrm{CH}_{2}$ ), 1.25-1.47 (m, 34H, $\left.\mathrm{CH}_{2}\right), 1.15-1.17$ (m, 21H, TIPS), 0.88 (t, $\left.J=6.9 \mathrm{~Hz}, 3 \mathrm{H}, \mathrm{CH}_{2} \mathrm{CH}_{3}\right) .{ }^{13} \mathrm{C} \mathrm{NMR}\left(126 \mathrm{MHz}, \mathrm{CDCl}_{3}, 25{ }^{\circ} \mathrm{C}\right): \delta 166.30,154.99,151.14,147.05$, $145.77,132.77,132.03,130.73,124.72,123.14,120.49,116.04,103.30,95.15,95.09$, 94.95, 65.34, 56.23, 56.11, 31.93, 29.71, 29.61, 29.58, 29.45, 29.37, 29.32, 28.74, 26.04, 22.70, 18.67, 14.13, 11.29. HRMS (ESI+): $m / z$ calcd for $\mathrm{C}_{47} \mathrm{H}_{77} \mathrm{NNaO}_{6} \mathrm{Si}\left(\mathrm{M}+\mathrm{Na}^{+}\right), 802.5412$; found 802.5407. 
Synthesis of 10k. 10j $(0.90 \mathrm{~g}, 1.2 \mathrm{mmol})$ was dissolved in anhydrous dichloromethane $(12 \mathrm{~mL})$

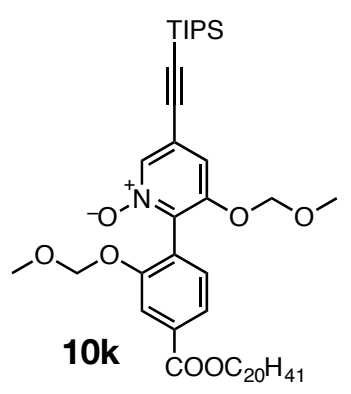
and the solution was cooled to $0{ }^{\circ} \mathrm{C}$. To this was added 3-chloroperoxybenzoic acid ( $m$ CPBA) (0.40 g, $2.3 \mathrm{mmol})$ dissolved in anhydrous dichloromethane $(4.6 \mathrm{~mL})$, and the mixture was stirred at room temperature for $15 \mathrm{~h}$. The mixture was diluted with chloroform and the solution was washed with saturated aqueous $\mathrm{NaHCO}_{3}$ and brine, and then dried over $\mathrm{Na}_{2} \mathrm{SO}_{4}$. The solvent was removed under reduced pressure and the residual compound was passed through a short pad of amino-functionalized silica gel using $n$-hexane/chloroform $(2 / 1, \mathrm{v} / \mathrm{v})$ as the eluent to give the desired product as a white solid (0.93 g, 99\% yield). Mp: 60.2-61.2 ${ }^{\circ} \mathrm{C}$. IR ( $\mathrm{KBr}$, $\left.\mathrm{cm}^{-1}\right): 2153(\mathrm{C} \equiv \mathrm{C}), 1715(\mathrm{C}=\mathrm{O}), 1248(\mathrm{~N}=\mathrm{O}) .{ }^{1} \mathrm{H}$ NMR $\left(500 \mathrm{MHz}, \mathrm{CDCl}_{3}, 25{ }^{\circ} \mathrm{C}\right): \delta 8.18(\mathrm{~d}, J=1.2$ $\mathrm{Hz}, 1 \mathrm{H}, \mathrm{Ar}-\mathrm{H}), 7.91$ (d, $J=1.5 \mathrm{~Hz}, 1 \mathrm{H}, \mathrm{Ar}-\mathrm{H}), 7.80$ (dd, $J=7.9,1.5 \mathrm{~Hz}, 1 \mathrm{H}, \mathrm{Ar}-\mathrm{H}), 7.31$ (d, $J=7.9$ $\mathrm{Hz}, 1 \mathrm{H}, \mathrm{Ar}-\mathrm{H}), 7.19$ (d, $J=1.5 \mathrm{~Hz}, 1 \mathrm{H}, \mathrm{Ar}-\mathrm{H}), 5.22$ (d, $\left.J=6.5 \mathrm{~Hz}, 1 \mathrm{H}, \mathrm{OCH}_{2} \mathrm{O}\right), 5.14$ (d, $J=6.8$ $\left.\mathrm{Hz}, 1 \mathrm{H}, \mathrm{OCH}_{2} \mathrm{O}\right), 5.12\left(\mathrm{~d}, J=6.8 \mathrm{~Hz}, 1 \mathrm{H}, \mathrm{OCH}_{2} \mathrm{O}\right), 5.09\left(\mathrm{~d}, J=7.1 \mathrm{~Hz}, 1 \mathrm{H}, \mathrm{OCH}_{2} \mathrm{O}\right), 4.33(\mathrm{t}, J=$ $\left.6.8 \mathrm{~Hz}, 2 \mathrm{H}, \mathrm{OCH}_{2} \mathrm{CH}_{2}\right), 3.41\left(\mathrm{~s}, 3 \mathrm{H}, \mathrm{OCH}_{3}\right), 3.37\left(\mathrm{~s}, 3 \mathrm{H}, \mathrm{OCH}_{3}\right), 1.76$ (quint, $J=7.7 \mathrm{~Hz}, 2 \mathrm{H}$, $\mathrm{OCH}_{2} \mathrm{CH}_{2}$ ), 1.22-1.46 (m, 34H, $\mathrm{CH}_{2}$ ), 1.12-1.15 (m, 21H, TIPS), 0.88 (t, J=7.1 Hz, 3H, $\mathrm{CH}_{2} \mathrm{CH}_{3}$ ). ${ }^{13} \mathrm{C}$ NMR (126 MHz, $\left.\mathrm{CDCl}_{3}, 25{ }^{\circ} \mathrm{C}\right): \delta 166.09,155.36,153.57,139.22,136.82,133.05,131.01$, 123.94, 123.10, 121.48, 115.96, 114.77, 100.85, 97.08, 95.04, 94.90, 65.39, 56.51, 56.18, 31.93, 29.71, 29.61, 29.58, 29.37, 29.32, 28.75, 26.02, 22.70, 18.60, 14.13, 11.17. HRMS (ESI+): $\mathrm{m} / z$ calcd for $\mathrm{C}_{47} \mathrm{H}_{77} \mathrm{NNaO}_{7} \mathrm{Si}\left(\mathrm{M}+\mathrm{Na}^{+}\right), 818.5367$; found 818.5339.

Synthesis of $\mathrm{rac}-\mathbf{2} \mathbf{a}^{\mathrm{NO}}$. To a solution of $\mathbf{1 0 k}(0.90 \mathrm{~g}, 1.13 \mathrm{mmol})$ in anhydrous THF $(45 \mathrm{~mL})$ was<smiles>C#Cc1cc(OCOC)c(-c2ccc(OC(C)(C)C)cc2OCOC)c(OC)n1</smiles>
added tetra- $n$-butylammonium fluoride (TBAF) $(1.0 \mathrm{M}$ in THF, $1.35 \mathrm{~mL}$, $1.35 \mathrm{mmol}$ ) at $0{ }^{\circ} \mathrm{C}$ and the mixture was stirred at $0{ }^{\circ} \mathrm{C}$ for $1 \mathrm{~h}$. After evaporating the solvent, the residue was diluted with chloroform and the solution was washed with water and brine, and then dried over $\mathrm{Na}_{2} \mathrm{SO}_{4}$. The solvent was removed under reduced pressure and the crude product was purified by silica gel chromatography using $n$-hexane/ethyl acetate $(3 / 7, \mathrm{v} / \mathrm{v})$ as the eluent to give the desired product as a white solid $\left(0.57 \mathrm{~g}, 79 \%\right.$ yield). $\mathrm{Mp}: 77.9-78.9^{\circ} \mathrm{C}$. IR $\left(\mathrm{KBr}, \mathrm{cm}^{-1}\right): 3117(\equiv \mathrm{CH})$, $2102(\mathrm{C} \equiv \mathrm{C}), 1714(\mathrm{C}=\mathrm{O}), 1255(\mathrm{~N}=\mathrm{O}) .{ }^{1} \mathrm{H} \mathrm{NMR}\left(500 \mathrm{MHz}, \mathrm{CDCl}_{3}, 50{ }^{\circ} \mathrm{C}\right): \delta 8.17(\mathrm{~d}, J=1.2 \mathrm{~Hz}$, 1H, Ar-H), 7.91 (d, $J=1.2 \mathrm{~Hz}, 1 \mathrm{H}, \mathrm{Ar}-\mathrm{H}), 7.81(\mathrm{dd}, J=7.9,1.5 \mathrm{~Hz}, 1 \mathrm{H}, \mathrm{Ar}-\mathrm{H}), 7.32$ (d, J= 7.9 Hz, $1 \mathrm{H}, \mathrm{Ar}-\mathrm{H}), 7.25(\mathrm{~d}, J=1.2 \mathrm{~Hz}, 1 \mathrm{H}, \mathrm{Ar}-\mathrm{H}), 5.22\left(\mathrm{~d}, J=6.8 \mathrm{~Hz}, 1 \mathrm{H}, \mathrm{OCH}_{2} \mathrm{O}\right), 5.14(\mathrm{~d}, J=6.5 \mathrm{~Hz}$, $\left.1 \mathrm{H}, \mathrm{OCH}_{2} \mathrm{O}\right), 5.11\left(\mathrm{~d}, J=7.1 \mathrm{~Hz}, 1 \mathrm{H}, \mathrm{OCH}_{2} \mathrm{O}\right), 5.08\left(\mathrm{~d}, J=6.8 \mathrm{~Hz}, 1 \mathrm{H}, \mathrm{OCH}_{2} \mathrm{O}\right), 4.33(\mathrm{t}, J=6.8 \mathrm{~Hz}$, $\left.2 \mathrm{H}, \mathrm{OCH}_{2} \mathrm{CH}_{2}\right), 3.40\left(\mathrm{~s}, 3 \mathrm{H}, \mathrm{OCH}_{3}\right), 3.37\left(\mathrm{~s}, 3 \mathrm{H}, \mathrm{OCH}_{3}\right), 3.26(\mathrm{~s}, 1 \mathrm{H}, \mathrm{C} \equiv \mathrm{C}-\mathrm{H}), 1.76$ (quint, $J=7.7$ $\left.\mathrm{Hz}, 2 \mathrm{H}, \mathrm{OCH}_{2} \mathrm{CH}_{2}\right), 1.22-1.46\left(\mathrm{~m}, 34 \mathrm{H}, \mathrm{CH}_{2}\right), 0.88\left(\mathrm{t}, J=7.1 \mathrm{~Hz}, 3 \mathrm{H}, \mathrm{CH}_{2} \mathrm{CH}_{3}\right) .{ }^{13} \mathrm{C} \mathrm{NMR}(126$ $\left.\mathrm{MHz}, \mathrm{CDCl}_{3}, 50{ }^{\circ} \mathrm{C}\right): \delta 166.06,155.33,153.63,139.79,136.67,133.12,130.96,123.79,123.11$, 
120.21, 116.01, 115.14, 95.09, 94.96, 81.90, 78.12, 65.40, 56.50, 56.17, 31.93, 29.71, 29.61, 29.58, 29.37, 29.31, 28.74, 26.01, 22.70, 14.13. HRMS $(\mathrm{ESI}+)$ : $m / z$ calcd for $\mathrm{C}_{38} \mathrm{H}_{57} \mathrm{NNaO}_{7}\left(\mathrm{M}+\mathrm{Na}^{+}\right)$, 662.4027; found 662.4039.

rac-2 $\mathbf{a}^{\mathbf{N O}}$ was resolved into enantiomers $\left((S)-\mathbf{2} \mathbf{a}^{\mathrm{NO}}\right.$ and $\left.(R)-\mathbf{2} \mathbf{a}^{\mathbf{N O}}\right)$ by chiral HPLC. Chiral HPLC conditions: column, CHIRALPAK IB $(2.0 \mathrm{~cm}$ (i.d.) $\times 20 \mathrm{~cm})$; eluent, $n$-hexane/chloroform/2propanol $(70 / 30 / 2, \mathrm{v} / \mathrm{v} / \mathrm{v})$; flow rate, $10 \mathrm{~mL} / \mathrm{min}$; temperature, $0{ }^{\circ} \mathrm{C}$. The absolute configurations were assigned based on the time-dependent-density functional theory (TD-DFT) calculations (see Section 3 and Figure S4).

Spectroscopic data of $(S)-2 \mathbf{a}^{\text {No: }}$. White solid. Mp: $69.9-70.1{ }^{\circ} \mathrm{C} .[\alpha]^{25} \mathrm{D}+24.3$ (c 1.0, chloroform).

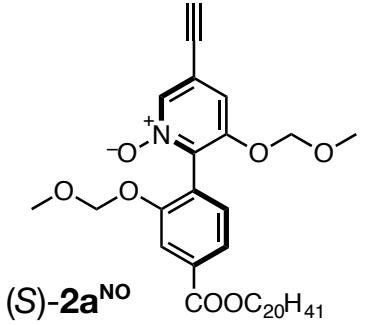
IR (KBr, cm $\left.{ }^{-1}\right): 3127(\equiv \mathrm{CH}), 2101(\mathrm{C} \equiv \mathrm{C}), 1714(\mathrm{C}=\mathrm{O}), 1254(\mathrm{~N}=\mathrm{O}) .{ }^{1} \mathrm{H}$ $\operatorname{NMR}\left(500 \mathrm{MHz}, \mathrm{CDCl}_{3}, 25^{\circ} \mathrm{C}\right): \delta 8.17(\mathrm{~d}, J=1.2 \mathrm{~Hz}, 1 \mathrm{H}, \mathrm{Ar}-\mathrm{H}), 7.91(\mathrm{~d}$, $J=1.5 \mathrm{~Hz}, 1 \mathrm{H}, \mathrm{Ar}-\mathrm{H}), 7.81(\mathrm{dd}, J=7.9,1.5 \mathrm{~Hz}, 1 \mathrm{H}, \mathrm{Ar}-\mathrm{H}), 7.32(\mathrm{~d}, J=$ $7.6 \mathrm{~Hz}, 1 \mathrm{H}, \mathrm{Ar}-\mathrm{H}), 7.25$ (partially overlapped with the signal derived from chloroform), $5.22\left(\mathrm{~d}, J=6.8 \mathrm{~Hz}, 1 \mathrm{H}, \mathrm{OCH}_{2} \mathrm{O}\right), 5.14(\mathrm{~d}, J=6.5 \mathrm{~Hz}, 1 \mathrm{H}$, $\left.\mathrm{OCH}_{2} \mathrm{O}\right), 5.11\left(\mathrm{~d}, J=7.1 \mathrm{~Hz}, 1 \mathrm{H}, \mathrm{OCH}_{2} \mathrm{O}\right), 5.08\left(\mathrm{~d}, J=6.8 \mathrm{~Hz}, 1 \mathrm{H}, \mathrm{OCH}_{2} \mathrm{O}\right), 4.33(\mathrm{t}, J=6.8 \mathrm{~Hz}, 2 \mathrm{H}$, $\left.\mathrm{OCH}_{2} \mathrm{CH}_{2}\right), 3.40\left(\mathrm{~s}, 3 \mathrm{H}, \mathrm{OCH}_{3}\right), 3.37\left(\mathrm{~s}, 3 \mathrm{H}, \mathrm{OCH}_{3}\right), 3.26(\mathrm{~s}, 1 \mathrm{H}, \mathrm{C} \equiv \mathrm{C}-\mathrm{H}), 1.76$ (quint, $J=7.7 \mathrm{~Hz}$, $\left.2 \mathrm{H}, \mathrm{OCH}_{2} \mathrm{CH}_{2}\right), 1.22-1.46\left(\mathrm{~m}, 34 \mathrm{H}, \mathrm{CH}_{2}\right), 0.88\left(\mathrm{t}, J=6.9 \mathrm{~Hz}, 3 \mathrm{H}, \mathrm{CH}_{2} \mathrm{CH}_{3}\right) .{ }^{13} \mathrm{C} \mathrm{NMR}(126 \mathrm{MHz}$, $\left.\mathrm{CDCl}_{3}, 25{ }^{\circ} \mathrm{C}\right): \delta 166.05,155.32,153.62,139.78,136.64,133.11,130.94,123.76,123.10,120.23$, 116.00, 115.19, 95.09, 94.96, 81.93, 78.10, 65.41, 56.50, 56.18, 31.93, 29.71, 29.61, 29.58, 29.37, 29.31, 28.75, 26.02, 22.70, 14.13. HRMS (ESI+): $m / z$ calcd for $\mathrm{C}_{38} \mathrm{H}_{57} \mathrm{NNaO}_{7}\left(\mathrm{M}+\mathrm{Na}^{+}\right), 662.4027$; found 662.4042 .

Spectroscopic data of $(R)-2 \mathbf{a}^{\text {No: }}$. White solid. Mp: 68.8-69.5 ${ }^{\circ} \mathrm{C} .[\alpha]^{25} \mathrm{D}-24.6(c 0.43$, chloroform).

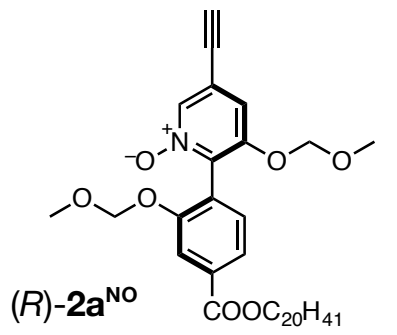
IR $\left(\mathrm{KBr}, \mathrm{cm}^{-1}\right): 3127(\equiv \mathrm{CH}), 2102(\mathrm{C} \equiv \mathrm{C}), 1714(\mathrm{C}=\mathrm{O}), 1253(\mathrm{~N}=\mathrm{O}) .{ }^{1} \mathrm{H}$ NMR $\left(500 \mathrm{MHz}, \mathrm{CDCl}_{3}, \mathrm{rt}\right): \delta 8.17(\mathrm{~d}, J=1.2 \mathrm{~Hz}, 1 \mathrm{H}, \mathrm{Ar}-\mathrm{H}), 7.91(\mathrm{~d}, J$ $=1.2 \mathrm{~Hz}, 1 \mathrm{H}, \mathrm{Ar}-\mathrm{H}), 7.81(\mathrm{dd}, J=7.9,1.5 \mathrm{~Hz}, 1 \mathrm{H}, \mathrm{Ar}-\mathrm{H}), 7.32(\mathrm{~d}, J=7.9$ $\mathrm{Hz}, 1 \mathrm{H}, \mathrm{Ar}-\mathrm{H}), 7.26(\mathrm{~d}, J=1.2 \mathrm{~Hz}, 1 \mathrm{H}, \mathrm{Ar}-\mathrm{H}), 5.22(\mathrm{~d}, J=6.7 \mathrm{~Hz}, 1 \mathrm{H}$, $\left.\mathrm{OCH}_{2} \mathrm{O}\right), 5.14\left(\mathrm{~d}, J=6.7 \mathrm{~Hz}, 1 \mathrm{H}, \mathrm{OCH}_{2} \mathrm{O}\right), 5.11(\mathrm{~d}, J=7.0 \mathrm{~Hz}, 1 \mathrm{H}$, $\left.\mathrm{OCH}_{2} \mathrm{O}\right), 5.08\left(\mathrm{~d}, J=7.0 \mathrm{~Hz}, 1 \mathrm{H}, \mathrm{OCH}_{2} \mathrm{O}\right), 4.33\left(\mathrm{t}, J=6.7 \mathrm{~Hz}, 2 \mathrm{H}, \mathrm{OCH}_{2} \mathrm{CH}_{2}\right), 3.40\left(\mathrm{~s}, 3 \mathrm{H}, \mathrm{OCH}_{3}\right)$, $3.37\left(\mathrm{~s}, 3 \mathrm{H}, \mathrm{OCH}_{3}\right), 3.26(\mathrm{~s}, 1 \mathrm{H}, \mathrm{C} \equiv \mathrm{C}-\mathrm{H}), 1.76$ (quint, $\left.J=7.7 \mathrm{~Hz}, 2 \mathrm{H}, \mathrm{OCH}_{2} \mathrm{CH}_{2}\right), 1.25-1.46(\mathrm{~m}, 34 \mathrm{H}$, $\left.\mathrm{CH}_{2}\right), 0.88\left(\mathrm{t}, J=7.0 \mathrm{~Hz}, 3 \mathrm{H}, \mathrm{CH}_{2} \mathrm{CH}_{3}\right) .{ }^{13} \mathrm{C} \mathrm{NMR}\left(126 \mathrm{MHz}, \mathrm{CDCl}_{3}, \mathrm{rt}\right): \delta 166.04,155.31,153.60$, 139.76, 136.64, 133.09, 130.94, 123.77, 123.09, 120.20, 115.99, 115.13, 95.07, 94.94, 81.90, 78.10, $65.39,56.49,56.16,31.93,29.71,29.61,29.58,29.37,29.30,28.74,26.01,22.70,14.13$. HRMS (ESI+): $m / z$ calcd for $\mathrm{C}_{38} \mathrm{H}_{57} \mathrm{NNaO}_{7}\left(\mathrm{M}+\mathrm{Na}^{+}\right), 662.4027$; found 662.4033. 


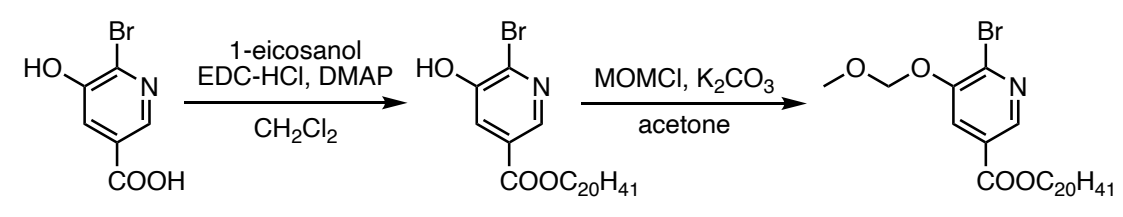

$11 \mathrm{a}$

11b

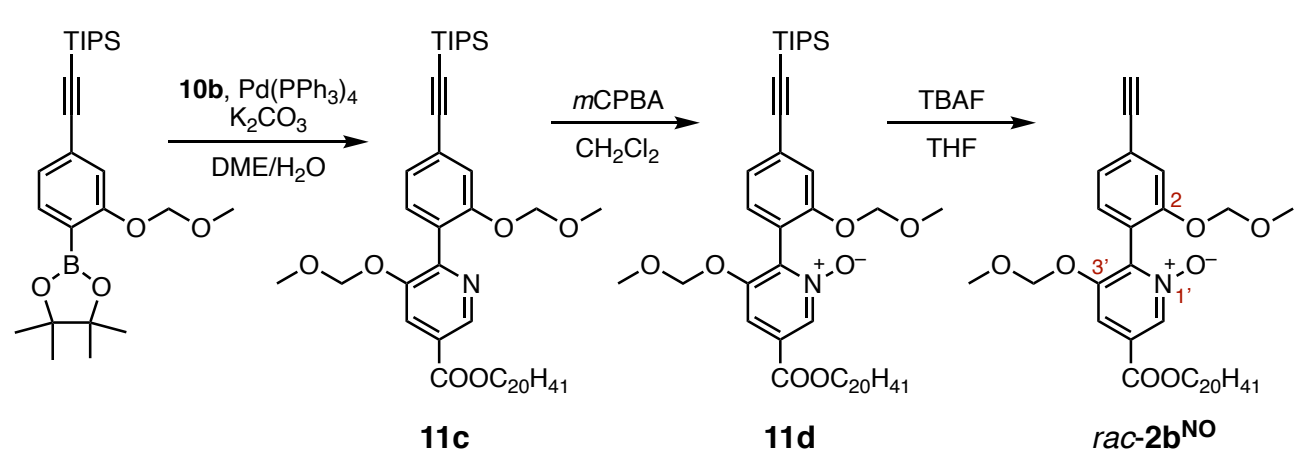

Scheme S2. Synthesis of $r a c-2 b^{\text {NO }}$.

Synthesis of 11c. To a mixture of 6-bromo-5-hydroxypyridine-3-carboxylic acid (425 mg, 1.95<smiles>CCOc1cnc(-c2ccc(C#CC(=O)OC)cc2OCOC)c(OCOC)c1</smiles>

11c mmol), DMAP (285 mg, $2.34 \mathrm{mmol}$ ), and 1-eicosanol (700 mg, $2.34 \mathrm{mmol}$ ) in anhydrous dichloromethane (4 mL) was added EDC-HCl (450 mg, $2.34 \mathrm{mmol}$ ) at $0{ }^{\circ} \mathrm{C}$. The mixture was stirred at room temperature for $18 \mathrm{~h}$. After evaporating the solvent, the residue was diluted with chloroform and the solution was washed with aqueous $1 \mathrm{~N} \mathrm{HCl}$, aqueous $1 \mathrm{~N} \mathrm{NaOH}$, and brine, and then dried over $\mathrm{Na}_{2} \mathrm{SO}_{4}$. After the solvent was evaporated to dryness under reduced pressure, the residue was passed through a short pad of silica gel using $n$ hexane/ethyl acetate $(10 / 1, v / v)$ as the eluent. After concentrating in vacuo, the crude product 11a (333 mg) was obtained as a white solid and was used without further purification. A mixture of the obtained 11a (285 mg), potassium carbonate (200 mg, $1.45 \mathrm{mmol})$, and chloromethyl methyl ether $(50 \mu \mathrm{L}, 0.66 \mathrm{mmol})$ in anhydrous acetone $(2.5 \mathrm{~mL})$ was stirred at $70{ }^{\circ} \mathrm{C}$ for $2 \mathrm{~h}$. After evaporating the solvent, the residue was diluted with ethyl acetate and the solution was washed with water and brine, and then dried over $\mathrm{Na}_{2} \mathrm{SO}_{4}$. After the solvent was evaporated to dryness under reduced pressure, the residue was passed through a short pad of silica gel using $n$-hexane/ethyl acetate $(7 / 1, \mathrm{v} / \mathrm{v})$ as the eluent. After concentrating in vacuo, the crude product $\mathbf{1 1 b}(132 \mathrm{mg})$ was obtained as a white solid and was used without further purification. A mixture of the obtained 11b (122 mg), 2methoxymethoxy-4-((triisopropylsilyl)ethynyl)phenylboronic acid pinacol ester (129 mg, 0.29 mmol), potassium carbonate (100 g, $0.72 \mathrm{mmol})$, and $\mathrm{Pd}\left(\mathrm{PPh}_{3}\right)_{4}(25 \mathrm{mg}, 0.022 \mathrm{mmol})$ in a degassed DME/water mixture $(3 / 1, \mathrm{v} / \mathrm{v} ; 8 \mathrm{~mL})$ was stirred at $80^{\circ} \mathrm{C}$ for $5 \mathrm{~h}$ under nitrogen. After evaporating the solvent, the residue was diluted with ethyl acetate and the solution was washed with water and brine, and then dried over $\mathrm{Na}_{2} \mathrm{SO}_{4}$. The solvent was removed under reduced pressure and the crude 
product was purified by silica gel chromatography using $n$-hexane/ethyl acetate $(4 / 1, \mathrm{v} / \mathrm{v})$ as the eluent to give the desired product as a pale yellow solid (112 mg, 9\% yield over 3 steps). ${ }^{1} \mathrm{H}$ NMR $\left(500 \mathrm{MHz}, \mathrm{CDCl}_{3}, 25^{\circ} \mathrm{C}\right): \delta 8.94(\mathrm{~d}, J=1.5 \mathrm{~Hz}, 1 \mathrm{H}, \mathrm{Ar}-\mathrm{H}), 8.08(\mathrm{~d}, J=1.8 \mathrm{~Hz}, 1 \mathrm{H}, \mathrm{Ar}-\mathrm{H}), 7.30(\mathrm{~d}$, $J=1.2 \mathrm{~Hz}, 1 \mathrm{H}, \mathrm{Ar}-\mathrm{H}), 7.28(\mathrm{~d}, J=7.6 \mathrm{~Hz}, 1 \mathrm{H}, \mathrm{Ar}-\mathrm{H}), 7.24(\mathrm{dd}, J=7.8,1.3 \mathrm{~Hz}, 1 \mathrm{H}, \mathrm{Ar}-\mathrm{H}), 5.15$ (s, $\left.2 \mathrm{H}, \mathrm{OCH}_{2} \mathrm{O}\right), 5.09\left(\mathrm{~s}, 2 \mathrm{H}, \mathrm{OCH}_{2} \mathrm{O}\right), 4.36\left(\mathrm{t}, J=6.8 \mathrm{~Hz}, 2 \mathrm{H}, \mathrm{OCH}_{2} \mathrm{CH}_{2}\right), 3.38\left(\mathrm{~s}, 3 \mathrm{H}, \mathrm{OCH}_{3}\right), 3.35(\mathrm{~s}$, $3 \mathrm{H}, \mathrm{OCH}_{3}$ ), 1.79 (quint, $\left.J=7.0 \mathrm{~Hz}, 2 \mathrm{H}, \mathrm{OCH}_{2} \mathrm{CH}_{2}\right), 1.22-1.46\left(\mathrm{~m}, 34 \mathrm{H}, \mathrm{CH}_{2}\right), 1.14$ (s, 21H, TIPS), $0.88\left(\mathrm{t}, J=7.1 \mathrm{~Hz}, 3 \mathrm{H}, \mathrm{CH}_{2} \mathrm{CH}_{3}\right)$.

Synthesis of 11d. 11c (110 mg, $0.141 \mathrm{mmol})$ was dissolved in anhydrous dichloromethane (1.5

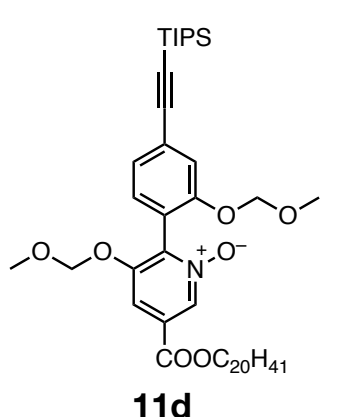
$\mathrm{mL}$ ) and the solution was cooled to $0^{\circ} \mathrm{C}$. To this was added $m \mathrm{CPBA}(50 \mathrm{mg}$, $0.29 \mathrm{mmol})$ dissolved in anhydrous dichloromethane $(0.50 \mathrm{~mL})$, and the mixture was stirred at room temperature for $9 \mathrm{~h}$. The mixture was diluted with chloroform and the solution was washed with saturated aqueous $\mathrm{NaHCO}_{3}$ and brine, and then dried over $\mathrm{Na}_{2} \mathrm{SO}_{4}$. The solvent was removed under reduced pressure and the residual compound was passed through a short pad of aminofunctionalized silica gel using $n$-hexane/chloroform $(1 / 1, \mathrm{v} / \mathrm{v})$ as the eluent to give the desired product as a pale yellow solid (94 mg, 84\% yield). ${ }^{1} \mathrm{H}$ NMR $\left(500 \mathrm{MHz}, \mathrm{CDCl}_{3}\right.$, $\left.25^{\circ} \mathrm{C}\right): \delta 8.66(\mathrm{~d}, J=1.5 \mathrm{~Hz}, 1 \mathrm{H}, \mathrm{Ar}-\mathrm{H}), 7.70(\mathrm{~d}, J=1.2 \mathrm{~Hz}, 1 \mathrm{H}, \mathrm{Ar}-\mathrm{H}), 7.35(\mathrm{~d}, J=1.2 \mathrm{~Hz}, 1 \mathrm{H}$, $\operatorname{Ar}-\mathrm{H}), 7.26(\mathrm{dd}, J=7.8,1.3 \mathrm{~Hz}, 1 \mathrm{H}, \mathrm{Ar}-\mathrm{H}), 7.19$ (d, $J=7.6 \mathrm{~Hz}, 1 \mathrm{H}, \mathrm{Ar}-\mathrm{H}), 5.09-5.17$ (m, 4H, $\left.\mathrm{OCH}_{2} \mathrm{O}\right), 4.35\left(\mathrm{t}, J=6.6 \mathrm{~Hz}, 2 \mathrm{H}, \mathrm{OCH}_{2} \mathrm{CH}_{2}\right), 3.40\left(\mathrm{~s}, 3 \mathrm{H}, \mathrm{OCH}_{3}\right), 3.36\left(\mathrm{~s}, 3 \mathrm{H}, \mathrm{OCH}_{3}\right), 1.75$ (quint, $J$ $\left.=7.0 \mathrm{~Hz}, 2 \mathrm{H}, \mathrm{OCH}_{2} \mathrm{CH}_{2}\right), 1.22-1.44\left(\mathrm{~m}, 34 \mathrm{H}, \mathrm{CH}_{2}\right), 1.13(\mathrm{~s}, 21 \mathrm{H}, \mathrm{TIPS}), 0.88$ (t, $J=7.1 \mathrm{~Hz}, 3 \mathrm{H}$, $\left.\mathrm{CH}_{2} \mathrm{CH}_{3}\right)$.

Synthesis of $\mathbf{r a c}-\mathbf{2} \mathbf{b}^{\mathrm{NO}}$. To a solution of $\mathbf{1 1 d}(92 \mathrm{mg}, 0.12 \mathrm{mmol})$ in anhydrous THF (4.5 mL) was

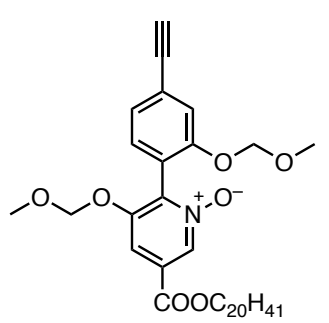

$r a c-2 b^{\text {NO }}$ added TBAF (1.0 M in THF, $0.14 \mathrm{~mL}, 0.14 \mathrm{mmol})$ at $0{ }^{\circ} \mathrm{C}$ and the mixture was stirred at $0{ }^{\circ} \mathrm{C}$ for $1 \mathrm{~h}$. After evaporating the solvent, the residue was diluted with chloroform and the solution was washed with water and brine, and then dried over $\mathrm{Na}_{2} \mathrm{SO}_{4}$. The solvent was removed under reduced pressure and the crude product was purified by silica gel chromatography using chloroform/ethyl acetate $(1 / 1, \mathrm{v} / \mathrm{v})$ as the eluent to give the desired product as a white solid (74 $\mathrm{mg}, 98 \%$ yield). Mp: 74.2-74.9 ${ }^{\circ} \mathrm{C}$. IR ( $\left.\mathrm{KBr}, \mathrm{cm}^{-1}\right): 3126(\equiv \mathrm{CH}), 2109$ $(\mathrm{C} \equiv \mathrm{C}), 1736(\mathrm{C}=\mathrm{O}), 1245(\mathrm{~N}=\mathrm{O}) .{ }^{1} \mathrm{H} \mathrm{NMR}\left(500 \mathrm{MHz}, \mathrm{CDCl}_{3}, 25^{\circ} \mathrm{C}\right): \delta 8.66(\mathrm{~d}, J=1.5 \mathrm{~Hz}, 1 \mathrm{H}, \mathrm{Ar}-$ H), $7.70(\mathrm{~d}, J=1.2 \mathrm{~Hz}, 1 \mathrm{H}, \mathrm{Ar}-\mathrm{H}), 7.42$ (d, $J=1.2 \mathrm{~Hz}, 1 \mathrm{H}, \mathrm{Ar}-\mathrm{H}), 7.26-7.28(\mathrm{~m}, 1 \mathrm{H}, \mathrm{Ar}-\mathrm{H}), 7.21$ $(\mathrm{d}, J=7.6 \mathrm{~Hz}, 1 \mathrm{H}, \mathrm{Ar}-\mathrm{H}), 5.08-5.17\left(\mathrm{~m}, 4 \mathrm{H}, \mathrm{OCH}_{2} \mathrm{O}\right), 4.35\left(\mathrm{t}, J=6.6 \mathrm{~Hz}, 2 \mathrm{H}, \mathrm{OCH}_{2} \mathrm{CH}_{2}\right), 3.40(\mathrm{~s}$, $\left.3 \mathrm{H}, \mathrm{OCH}_{3}\right), 3.36\left(\mathrm{~s}, 3 \mathrm{H}, \mathrm{OCH}_{3}\right), 3.12(\mathrm{~s}, 1 \mathrm{H}, \mathrm{C} \equiv \mathrm{C}-\mathrm{H}), 1.76$ (quint, $J=7.0 \mathrm{~Hz}, 2 \mathrm{H}, \mathrm{OCH}_{2} \mathrm{CH}_{2}$ ), $1.22-$ 
$1.45\left(\mathrm{~m}, 34 \mathrm{H}, \mathrm{CH}_{2}\right), 0.88\left(\mathrm{t}, J=7.1 \mathrm{~Hz}, 3 \mathrm{H}, \mathrm{CH}_{2} \mathrm{CH}_{3}\right) .{ }^{13} \mathrm{C} \mathrm{NMR}\left(126 \mathrm{MHz}, \mathrm{CDCl}_{3}, 25{ }^{\circ} \mathrm{C}\right): \delta 163.27$, $155.15,153.75,142.61,135.15,130.71,128.19,125.86,124.89,120.04,118.81,112.48,95.07,95.02$, 83.21, 78.11, 66.41, 56.54, 56.09, 31.93, 29.71, 29.68, 29.66, 29.60, 29.53, 29.36, 29.26, 28.58, 25.94, 22.69, 14.12. HRMS (ESI+): $m / z$ calcd for $\mathrm{C}_{38} \mathrm{H}_{57} \mathrm{NNaO}_{7}\left(\mathrm{M}+\mathrm{Na}^{+}\right), 662.4027$; found 662.4032 .

rac-2 $\mathbf{b}^{\text {NO }}$ was resolved into enantiomers $\left((S)-\mathbf{2} \mathbf{b}^{\mathbf{N O}}\right.$ and $\left.(R)-\mathbf{2} \mathbf{b}^{\mathbf{N O}}\right)$ by chiral HPLC. Chiral HPLC conditions: column, CHIRALPAK IB $(2.0 \mathrm{~cm}$ (i.d.) $\times 20 \mathrm{~cm})$; eluent, $n$-hexane/chloroform/2propanol $(70 / 30 / 2, \mathrm{v} / \mathrm{v} / \mathrm{v})$; flow rate, $10 \mathrm{~mL} / \mathrm{min}$; temperature, $0{ }^{\circ} \mathrm{C}$. The absolute configurations were assigned based on the TD-DFT calculations (see Section 3 and Figure S5).

Spectroscopic data of $(S)-2 \mathbf{b}^{\text {NO}}$ : White solid. Mp: 68.3-69.2 ${ }^{\circ} \mathrm{C}$. $[\alpha]^{25} \mathrm{D}+28.4(c 0.42$, chloroform).

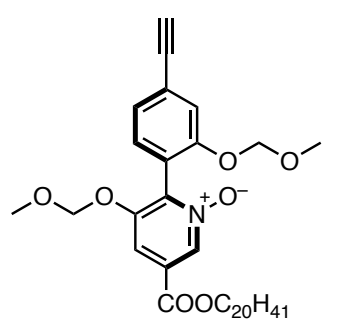

(S) $-2 \mathbf{b}^{\mathrm{NO}}$ IR $\left(\mathrm{KBr}, \mathrm{cm}^{-1}\right): 3126(\equiv \mathrm{CH}), 2109(\mathrm{C} \equiv \mathrm{C}), 1736(\mathrm{C}=\mathrm{O}), 1245(\mathrm{~N}=\mathrm{O}) .{ }^{1} \mathrm{H}$ NMR $\left(500 \mathrm{MHz}, \mathrm{CDCl}_{3}, 25^{\circ} \mathrm{C}\right): \delta 8.66(\mathrm{~d}, J=1.5 \mathrm{~Hz}, 1 \mathrm{H}, \mathrm{Ar}-\mathrm{H}), 7.70(\mathrm{~d}$, $J=1.2 \mathrm{~Hz}, 1 \mathrm{H}, \mathrm{Ar}-\mathrm{H}), 7.42(\mathrm{~d}, J=1.2 \mathrm{~Hz}, 1 \mathrm{H}, \mathrm{Ar}-\mathrm{H}), 7.26-7.28(\mathrm{~m}, 1 \mathrm{H}$, $\mathrm{Ar}-\mathrm{H}), 7.21(\mathrm{~d}, J=7.8 \mathrm{~Hz}, 1 \mathrm{H}, \mathrm{Ar}-\mathrm{H}), 5.08-5.17\left(\mathrm{~m}, 4 \mathrm{H}, \mathrm{OCH}_{2} \mathrm{O}\right), 4.35$ (t, $\left.J=6.7 \mathrm{~Hz}, 2 \mathrm{H}, \mathrm{OCH}_{2} \mathrm{CH}_{2}\right), 3.40\left(\mathrm{~s}, 3 \mathrm{H}, \mathrm{OCH}_{3}\right), 3.36\left(\mathrm{~s}, 3 \mathrm{H}, \mathrm{OCH}_{3}\right), 3.12(\mathrm{~s}$, $1 \mathrm{H}, \mathrm{C} \equiv \mathrm{C}-\mathrm{H}$ ), 1.76 (quint, $\left.J=7.0 \mathrm{~Hz}, 2 \mathrm{H}, \mathrm{OCH}_{2} \mathrm{CH}_{2}\right), 1.22-1.45(\mathrm{~m}, 34 \mathrm{H}$, $\left.\mathrm{CH}_{2}\right), 0.88$ (t, $\left.J=7.0 \mathrm{~Hz}, 3 \mathrm{H}, \mathrm{CH}_{2} \mathrm{CH}_{3}\right) .{ }^{13} \mathrm{C} \mathrm{NMR}\left(126 \mathrm{MHz}, \mathrm{CDCl}_{3}, 25{ }^{\circ} \mathrm{C}\right): \delta 163.28,155.15$, $153.75,142.61,135.16,130.72,128.19,125.87,124.89,120.05,118.82,112.47,95.07,95.02,83.22$, 78.11, 66.41, 56.54, 56.09, 31.93, 29.71, 29.66, 29.60, 29.53, 29.36, 29.26, 28.59, 25.94, 22.69, 14.12 . HRMS (ESI+): $m / z$ calcd for $\mathrm{C}_{38} \mathrm{H}_{57} \mathrm{NNaO}_{7}\left(\mathrm{M}+\mathrm{Na}^{+}\right), 662.4027$; found 662.4022 .

Spectroscopic data of $(R)-2 \mathbf{b}^{\text {No: }}$. White solid. Mp: 68.0-68. ${ }^{\circ} \mathrm{C}$. $[\alpha]^{25} \mathrm{D}-28.8$ (c 0.27, chloroform).

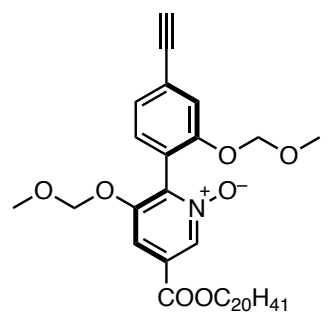

$(R)-2 \mathbf{b}^{\mathrm{NO}}$ IR $\left(\mathrm{KBr}, \mathrm{cm}^{-1}\right): 3127(\equiv \mathrm{CH}), 2109(\mathrm{C} \equiv \mathrm{C}), 1736(\mathrm{C}=\mathrm{O}), 1245(\mathrm{~N}=\mathrm{O}) .{ }^{1} \mathrm{H}$ $\operatorname{NMR}\left(500 \mathrm{MHz}, \mathrm{CDCl}_{3}, 25^{\circ} \mathrm{C}\right): \delta 8.66(\mathrm{~d}, J=1.2 \mathrm{~Hz}, 1 \mathrm{H}, \mathrm{Ar}-\mathrm{H}), 7.70(\mathrm{~d}$, $J=1.5 \mathrm{~Hz}, 1 \mathrm{H}, \mathrm{Ar}-\mathrm{H}), 7.42(\mathrm{~d}, J=1.2 \mathrm{~Hz}, 1 \mathrm{H}, \mathrm{Ar}-\mathrm{H}), 7.26-7.28(\mathrm{~m}, 1 \mathrm{H}$, $\mathrm{Ar}-\mathrm{H}), 7.21(\mathrm{~d}, J=7.8 \mathrm{~Hz}, 1 \mathrm{H}, \mathrm{Ar}-\mathrm{H}), 5.08-5.17\left(\mathrm{~m}, 4 \mathrm{H}, \mathrm{OCH}_{2} \mathrm{O}\right), 4.35$ (t, $\left.J=6.7 \mathrm{~Hz}, 2 \mathrm{H}, \mathrm{OCH}_{2} \mathrm{CH}_{2}\right), 3.40\left(\mathrm{~s}, 3 \mathrm{H}, \mathrm{OCH}_{3}\right), 3.36\left(\mathrm{~s}, 3 \mathrm{H}, \mathrm{OCH}_{3}\right), 3.12(\mathrm{~s}$, $1 \mathrm{H}, \mathrm{C} \equiv \mathrm{C}-\mathrm{H}$ ), 1.76 (quint, $\left.J=7.0 \mathrm{~Hz}, 2 \mathrm{H}, \mathrm{OCH}_{2} \mathrm{CH}_{2}\right), 1.22-1.45(\mathrm{~m}, 34 \mathrm{H}$, $\left.\mathrm{CH}_{2}\right), 0.88$ (t, $\left.J=7.0 \mathrm{~Hz}, 3 \mathrm{H}, \mathrm{CH}_{2} \mathrm{CH}_{3}\right) .{ }^{13} \mathrm{C} \mathrm{NMR}\left(126 \mathrm{MHz}, \mathrm{CDCl}_{3}, 25{ }^{\circ} \mathrm{C}\right): \delta 163.28,155.15$, $153.75,142.61,135.16,130.72,128.19,125.87,124.89,120.05,118.82,112.47,95.07,95.02,83.22$, 78.11, 66.41, 56.54, 56.10, 31.93, 29.71, 29.60, 29.53, 29.37, 29.26, 28.59, 25.95, 22.69, 14.12. HRMS (ESI+): $m / z$ calcd for $\mathrm{C}_{38} \mathrm{H}_{57} \mathrm{NNaO}_{7}\left(\mathrm{M}+\mathrm{Na}^{+}\right)$, 662.4027; found 662.4030 . 
Polymerization. Homopolymerization of $r a c-2 \mathbf{a}^{\mathbf{N O}},(S)-\mathbf{2} \mathbf{a}^{\mathbf{N O}},(R)-\mathbf{2} \mathbf{a}^{\mathbf{N O}}$, and $(R)-\mathbf{2} \mathbf{b}^{\mathbf{N O}}$, and copolymerization of $(S)-\mathbf{2} \mathbf{a}^{\mathbf{N O}}$ with $(R)-\mathbf{2} \mathbf{a}^{\mathbf{N O}}$ were carried out according to Scheme S3 in a dry glass ampule under a dry nitrogen atmosphere with a multicomponent catalytic system using $\left[(\text { norbornadiene)rhodium(I) chloride }]_{2}\left([\mathrm{Rh}(\mathrm{nbd}) \mathrm{Cl}]_{2}\right)\right.$ as a catalyst based on the reported method. ${ }^{\mathrm{S} 2}$ The results of the homo- and copolymerizations are summarized in Tables 1 and 2, respectively. A typical polymerization procedure is described below.
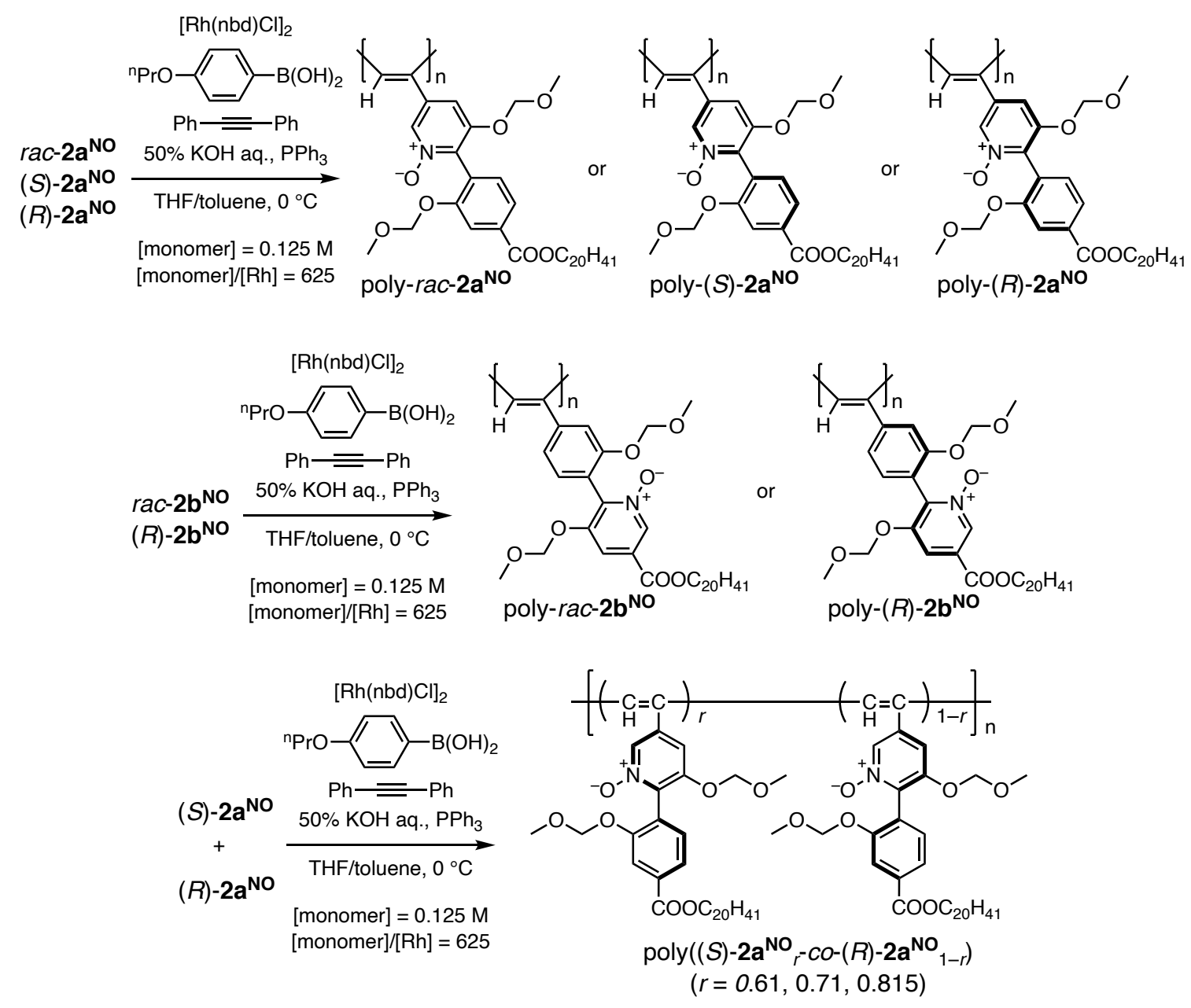

Scheme S3. Synthesis of poly-rac-2a ${ }^{\text {No }}$, poly- $(S)-\mathbf{2} \mathbf{a}^{\text {No }}$, poly- $(R)-\mathbf{2} \mathbf{a}^{\text {NO }}$, poly-rac-2 $\mathbf{b}^{\mathrm{NO}}$, poly- $(R)-\mathbf{2} \mathbf{b}^{\mathrm{NO}}$, and poly $\left((S)-\mathbf{2} \mathbf{a}^{\mathbf{N O}}{ }_{r-c o}-(R)-\mathbf{2} \mathbf{a}^{\mathbf{N O}}{ }_{1-r}\right)$.

A solution of $[\mathrm{Rh}(\mathrm{nbd}] \mathrm{Cl}]_{2}(12 \mathrm{mg}, 26 \mu \mathrm{mol}), 4$-propoxyphenylboronic acid (14 mg, $\left.78 \mu \mathrm{mol}\right)$, and diphenylacetylene $(37.1 \mathrm{mg}, 0.208 \mathrm{mmol})$ in THF $(260 \mu \mathrm{L})$ was cooled to $0{ }^{\circ} \mathrm{C}$. To this was added $50 \%(\mathrm{w} / \mathrm{v})$ aqueous $\mathrm{KOH}(15 \mu \mathrm{L}, 0.13 \mathrm{mmol})$, and the resulting solution was stirred at $0{ }^{\circ} \mathrm{C}$ for $5 \mathrm{~min}$. $\mathrm{PPh}_{3}(41 \mathrm{mg}, 0.16 \mathrm{mmol})$ was then added to this solution and the resulting catalyst solution was diluted with toluene $(24.7 \mathrm{~mL})$. A $50 \mu \mathrm{L}$ aliquot of the catalyst solution was rapidly added to a solution of $\mathrm{rac}-\mathbf{2 a}(40 \mathrm{mg}, 0.062 \mathrm{mmol})$ in toluene $(450 \mu \mathrm{L})$ at $0{ }^{\circ} \mathrm{C}$, and the reaction mixture was stirred at $0{ }^{\circ} \mathrm{C}$ for $24 \mathrm{~h}$. After excess acetic acid (a few drops) was added to the reaction mixture, the resulting polymer was precipitated into a large amount of methanol, collected by centrifugation, 
washed with methanol, and dried in vacuo at room temperature (35 mg, 88\% yield) (entry 1 in Table 1). The $M_{\mathrm{n}}$ and $M_{\mathrm{w}} / M_{\mathrm{n}}$ of the polymer were determined by SEC using polystyrene standards in THF containing $0.5 \mathrm{wt} \% \mathrm{TBAB}$. The cis-transoidal stereoregular structures of these polymers were confirmed by their ${ }^{1} \mathrm{H}$ NMR (Figures S60-S67) ${ }^{\mathrm{S} 3-7}$ and Raman spectra (Figures S24a and S26) ${ }^{\mathrm{S} 8-11}$ based on the literature.

Spectroscopic data of poly-rac-2a ${ }^{\text {NO}}$ : IR $\left(\mathrm{KBr}, \mathrm{cm}^{-1}\right)$ : $1720(\mathrm{C}=\mathrm{O}), 1229(\mathrm{~N}=\mathrm{O}) .{ }^{1} \mathrm{H}$ NMR (500

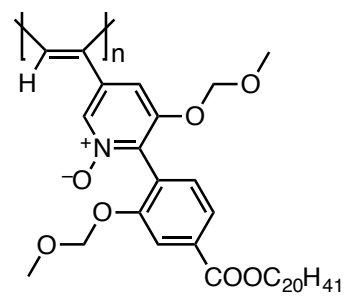

poly-rac-2a ${ }^{\mathrm{NO}}$ $\mathrm{MHz}, \mathrm{CDCl}_{3}, 50{ }^{\circ} \mathrm{C}$ ): $\delta 7.45-8.15$ (br, 3H, Ar-H), 6.80-7.30 (br, 2H, ArH), 6.20-6.60 (br, 1H, C=CH), 4.50-5.40 (br, 4H, $\left.\mathrm{OCH}_{2} \mathrm{O}\right), 4.00-4.50$ (br, $2 \mathrm{H}, \mathrm{COOCH}_{2}$ ), 2.90-3.50 (br, 6H, $\mathrm{OCH}_{3}$ ), 1.60-1.80 (br, $2 \mathrm{H}, \mathrm{OCH}_{2} \mathrm{CH}_{2}$ ), 1.15-1.40 (br, 34H, $\left.\mathrm{CH}_{2}\right), 0.87\left(\mathrm{t}, J=6.9 \mathrm{~Hz}, 3 \mathrm{H}, \mathrm{CH}_{3}\right)$. Calcd for $\mathrm{C}_{38} \mathrm{H}_{57} \mathrm{NO}_{7}: \mathrm{C}, 71.33 ; \mathrm{H}, 8.98 ; \mathrm{N}, 2.19$. Found: C, 71.30; H, 8.91; N, 2.26.

Spectroscopic data of poly-(S)-2a ${ }^{\text {NO}}$ : IR $\left(\mathrm{KBr}, \mathrm{cm}^{-1}\right): 1719(\mathrm{C}=\mathrm{O}), 1230(\mathrm{~N}=\mathrm{O}) .{ }^{1} \mathrm{H}$ NMR $(500$

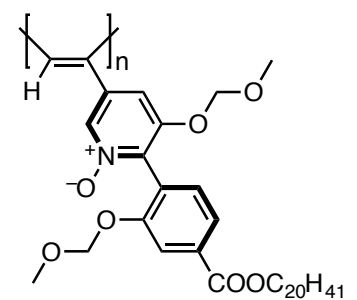

poly-(S)-2a $\mathrm{a}^{\text {NO }}$

$\left.\mathrm{MHz}, \mathrm{CDCl}_{3}, 50{ }^{\circ} \mathrm{C}\right): \delta 7.45-8.15$ (br, $\left.3 \mathrm{H}, \mathrm{Ar}-\mathrm{H}\right), 6.80-7.30$ (br, $2 \mathrm{H}, \mathrm{Ar}-$ $\mathrm{H}), 6.20-6.60$ (br, $1 \mathrm{H}, \mathrm{C}=\mathrm{CH}), 4.50-5.40\left(\mathrm{br}, 4 \mathrm{H}, \mathrm{OCH}_{2} \mathrm{O}\right), 4.00-4.50$ (br, $2 \mathrm{H}, \mathrm{COOCH}_{2}$ ), 2.90-3.50 (br, 6H, $\left.\mathrm{OCH}_{3}\right), 1.60-1.80\left(\mathrm{br}, 2 \mathrm{H}, \mathrm{OCH}_{2} \mathrm{CH}_{2}\right.$ ), 1.15-1.40 (br, 34H, $\left.\mathrm{CH}_{2}\right), 0.87\left(\mathrm{t}, J=6.9 \mathrm{~Hz}, 3 \mathrm{H}, \mathrm{CH}_{3}\right)$. Calcd for $\mathrm{C}_{38} \mathrm{H}_{57} \mathrm{NO}_{7}: \mathrm{C}, 71.33 ; \mathrm{H}, 8.98 ; \mathrm{N}, 2.19$. Found: C, 71.31; H, 9.00; N, 2.17.

Spectroscopic data of poly- $(R)-\mathbf{2 a}^{\mathrm{NO}}$ : IR $\left(\mathrm{KBr}, \mathrm{cm}^{-1}\right): 1719(\mathrm{C}=\mathrm{O}), 1229(\mathrm{~N}=\mathrm{O}) .{ }^{1} \mathrm{H}$ NMR (500

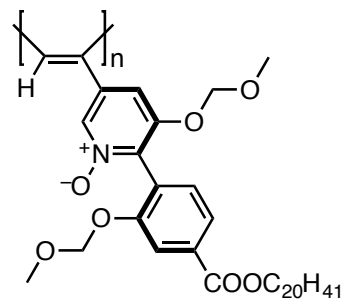

poly- $(R)-2 a^{\text {NO }}$ $\left.\mathrm{MHz}, \mathrm{CDCl}_{3}, 50{ }^{\circ} \mathrm{C}\right): \delta 7.45-8.15$ (br, $\left.3 \mathrm{H}, \mathrm{Ar}-\mathrm{H}\right), 6.80-7.30$ (br, $2 \mathrm{H}, \mathrm{Ar}-$ $\mathrm{H}), 6.20-6.60(\mathrm{br}, 1 \mathrm{H}, \mathrm{C}=\mathrm{CH}), 4.50-5.40\left(\mathrm{br}, 4 \mathrm{H}, \mathrm{OCH}_{2} \mathrm{O}\right), 4.00-4.50$ (br, $2 \mathrm{H}, \mathrm{COOCH}_{2}$ ), 2.90-3.50 (br, $\left.6 \mathrm{H}, \mathrm{OCH}_{3}\right), 1.60-1.80$ (br, $2 \mathrm{H}, \mathrm{OCH}_{2} \mathrm{CH}_{2}$ ), 1.15-1.40 (br, 34H, $\mathrm{CH}_{2}$ ), 0.87 (t, $J=6.9 \mathrm{~Hz}, 3 \mathrm{H}, \mathrm{CH}_{3}$ ). Calcd for $\mathrm{C}_{38} \mathrm{H}_{57} \mathrm{NO}_{7}$ : C, 71.33; H, 8.98; N, 2.19. Found: C, 71.35; H, 8.96; N, 2.17.

Spectroscopic data of poly-rac-2b ${ }^{\text {NO}}$ : IR $\left(\mathrm{KBr}, \mathrm{cm}^{-1}\right): 1719(\mathrm{C}=\mathrm{O}), 1230(\mathrm{~N}=\mathrm{O}) .{ }^{1} \mathrm{H}$ NMR (500

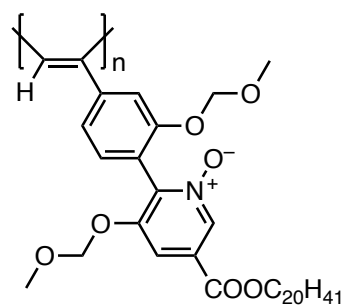

poly-rac-2 $\mathbf{b}^{\text {NO }}$ $\mathrm{MHz}, \mathrm{CDCl}_{3}, 50{ }^{\circ} \mathrm{C}$ ): $\delta 8.30-8.60$ (br, $\left.1 \mathrm{H}, \mathrm{Ar}-\mathrm{H}\right), 7.40-7.70$ (br, $1 \mathrm{H}, \mathrm{Ar}-$ H), 6.35-7.20 (br, 3H, Ar-H), 5.80-6.30 (br, 1H, C=CH), 4.45-5.20 (br, 4H, $\mathrm{OCH}_{2} \mathrm{O}$ ), 4.05-4.45 (br, 2H, $\mathrm{COOCH}_{2}$ ), 2.70-3.45 (br, 6H, $\mathrm{OCH}_{3}$ ), 1.60$1.80\left(\mathrm{br}, 2 \mathrm{H}, \mathrm{OCH}_{2} \mathrm{CH}_{2}\right.$ ), 1.15-1.40 (br, $\left.34 \mathrm{H}, \mathrm{CH}_{2}\right), 0.87$ (t, $J=6.8 \mathrm{~Hz}, 3 \mathrm{H}$, $\mathrm{CH}_{3}$ ). Calcd for $\mathrm{C}_{38} \mathrm{H}_{57} \mathrm{NO}_{7}$ : $\mathrm{C}, 71.33 ; \mathrm{H}, 8.98 ; \mathrm{N}, 2.19$. Found: $\mathrm{C}, 71.36$; $\mathrm{H}, 8.85 ; \mathrm{N}, 2.23$. 
Spectroscopic data of poly- $(R)-\mathbf{2} \mathbf{b}^{\mathrm{NO}}$ : IR $\left(\mathrm{KBr}, \mathrm{cm}^{-1}\right): 1719(\mathrm{C}=\mathrm{O}), 1230(\mathrm{~N}=\mathrm{O}) .{ }^{1} \mathrm{H}$ NMR $(500$

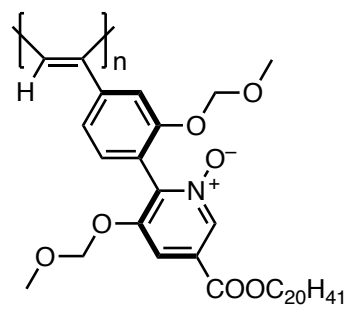

poly- $(R)-2 \mathbf{b}^{\text {NO }}$

$\mathrm{MHz}, \mathrm{CDCl}_{3}, 50{ }^{\circ} \mathrm{C}$ ): $\delta 8.30-8.55$ (br, $\left.1 \mathrm{H}, \mathrm{Ar}-\mathrm{H}\right), 7.40-7.70$ (br, $1 \mathrm{H}, \mathrm{Ar}-$ $\mathrm{H}), 6.35-7.20$ (br, 3H, Ar-H), 5.80-6.30 (br, 1H, C=CH), 4.45-5.20 (br, 4H, $\mathrm{OCH}_{2} \mathrm{O}$ ), 4.05-4.45 (br, 2H, $\mathrm{COOCH}_{2}$ ), 2.70-3.40 (br, 6H, $\mathrm{OCH}_{3}$ ), 1.60 1.80 (br, 2H, $\mathrm{OCH}_{2} \mathrm{CH}_{2}$ ), 1.15-1.40 (br, $\left.34 \mathrm{H}, \mathrm{CH}_{2}\right), 0.87$ (t, $J=6.8 \mathrm{~Hz}, 3 \mathrm{H}$, $\mathrm{CH}_{3}$ ). Calcd for $\mathrm{C}_{38} \mathrm{H}_{57} \mathrm{NO}_{7} \cdot 0.4 \mathrm{H}_{2} \mathrm{O}: \mathrm{C}, 70.54 ; \mathrm{H}, 9.00 ; \mathrm{N}, 2.16$. Found: $\mathrm{C}$, $70.52 ; \mathrm{H}, 8.72 ; \mathrm{N}, 1.67$.

Spectroscopic data of poly $\left((S)-\mathbf{2}^{\mathbf{N O}_{0.815}-c o-}(R)-\mathbf{2 a}^{\left.\mathbf{N O}_{0.185}\right):} \mathrm{IR}\left(\mathrm{KBr}, \mathrm{cm}^{-1}\right): 1719(\mathrm{C}=\mathrm{O}), 1230\right.$

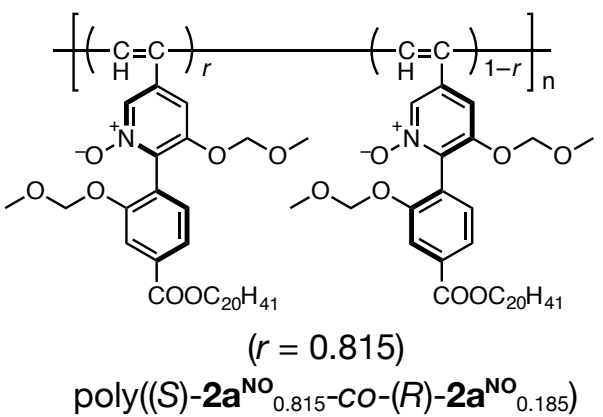

Spectroscopic data of poly $\left((S)-\mathbf{2 a}^{\mathbf{N O}_{0.71}-c o-}(R)-\mathbf{2 a}^{\mathbf{N O}}{ }_{0.29}\right): \operatorname{IR}\left(\mathrm{KBr}, \mathrm{cm}^{-1}\right): 1719(\mathrm{C}=\mathrm{O}), 1230$ $3 \mathrm{H}, \mathrm{Ar}-\mathrm{H}$ ), 6.80-7.30 (br, 2H, Ar-H), 6.20-6.60 (br, 1H, $\mathrm{C}=\mathrm{CH}), 4.50-5.40\left(\mathrm{br}, 4 \mathrm{H}, \mathrm{OCH}_{2} \mathrm{O}\right), 4.00-4.50(\mathrm{br}, 2 \mathrm{H}$, $\mathrm{COOCH}_{2}$ ), 2.90-3.50 (br, 6H, $\left.\mathrm{OCH}_{3}\right), 1.60-1.80$ (br, $2 \mathrm{H}$, $\mathrm{OCH}_{2} \mathrm{CH}_{2}$ ), 1.15-1.40 (br, 34H, $\mathrm{CH}_{2}$ ), 0.87 (t, $J=6.9 \mathrm{~Hz}$, $3 \mathrm{H}, \mathrm{CH}_{3}$ ). Calcd for $\mathrm{C}_{38} \mathrm{H}_{57} \mathrm{NO}_{7}: \mathrm{C}, 71.33 ; \mathrm{H}, 8.98 ; \mathrm{N}, 2.19$. Found: C, 71.31; H, 8.93; N, 2.23 . $(\mathrm{N}=\mathrm{O}) .{ }^{1} \mathrm{H}$ NMR $\left(500 \mathrm{MHz}, \mathrm{CDCl}_{3}, 50{ }^{\circ} \mathrm{C}\right): \delta 7.45-8.15(\mathrm{br}$,

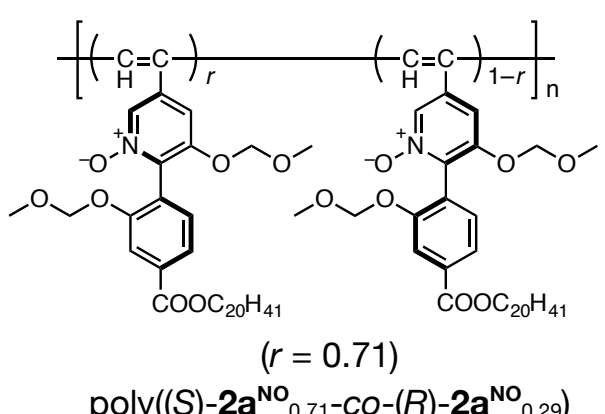

$(\mathrm{N}=\mathrm{O}) .{ }^{1} \mathrm{H}$ NMR $\left(500 \mathrm{MHz}, \mathrm{CDCl}_{3}, 50{ }^{\circ} \mathrm{C}\right): \delta 7.45-8.15(\mathrm{br}$, $3 \mathrm{H}, \mathrm{Ar}-\mathrm{H}), 6.80-7.30$ (br, 2H, Ar-H), 6.20-6.60 (br, 1H, $\mathrm{C}=\mathrm{CH}), 4.50-5.40\left(\mathrm{br}, 4 \mathrm{H}, \mathrm{OCH}_{2} \mathrm{O}\right), 4.00-4.50(\mathrm{br}, 2 \mathrm{H}$, $\mathrm{COOCH}_{2}$ ), 2.90-3.50 (br, 6H, $\mathrm{OCH}_{3}$ ), 1.60-1.80 (br, $2 \mathrm{H}$, $\mathrm{OCH}_{2} \mathrm{CH}_{2}$ ), $1.15-1.40\left(\mathrm{br}, 34 \mathrm{H}, \mathrm{CH}_{2}\right), 0.87$ (t, $J=6.9 \mathrm{~Hz}$, $3 \mathrm{H}, \mathrm{CH}_{3}$ ). Calcd for $\mathrm{C}_{38} \mathrm{H}_{57} \mathrm{NO}_{7}: \mathrm{C}, 71.33 ; \mathrm{H}, 8.98 ; \mathrm{N}, 2.19$. Found: C, 71.35; H, 8.96; N, 2.18 .

Spectroscopic data of poly $\left((S)-\mathbf{2 a}^{\mathbf{N O}}{ }_{0.61-c o}(R)-\mathbf{2 a}^{\mathbf{N O}}{ }_{0.39}\right):$ IR $\left(\mathrm{KBr}, \mathrm{cm}^{-1}\right): 1719(\mathrm{C}=\mathrm{O}), 1230$

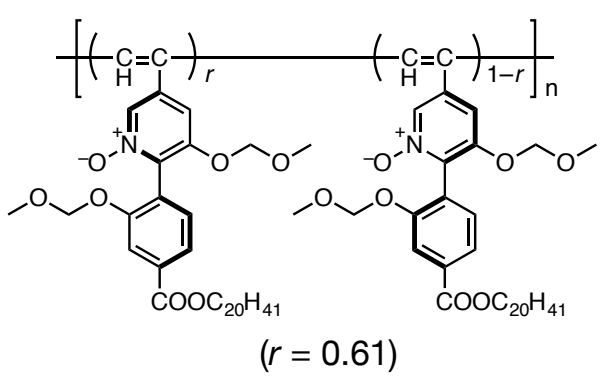

poly((S)-2a $\left.{ }^{\mathrm{NO}}{ }_{0.61}-\mathrm{CO}-(R)-2 \mathrm{a}^{\mathrm{NO}}{ }_{0.39}\right)$ $(\mathrm{N}=\mathrm{O}) .{ }^{1} \mathrm{H}$ NMR $\left(500 \mathrm{MHz}, \mathrm{CDCl}_{3}, 50{ }^{\circ} \mathrm{C}\right): \delta 7.45-8.15(\mathrm{br}$, $3 \mathrm{H}, \mathrm{Ar}-\mathrm{H}$ ), 6.80-7.30 (br, 2H, Ar-H), 6.20-6.60 (br, 1H, $\mathrm{C}=\mathrm{CH}), 4.50-5.40\left(\mathrm{br}, 4 \mathrm{H}, \mathrm{OCH}_{2} \mathrm{O}\right), 4.00-4.50(\mathrm{br}, 2 \mathrm{H}$, $\mathrm{COOCH}_{2}$ ), 2.90-3.50 (br, 6H, $\mathrm{OCH}_{3}$ ), 1.60-1.80 (br, $2 \mathrm{H}$, $\mathrm{OCH}_{2} \mathrm{CH}_{2}$ ), $1.15-1.40$ (br, 34H, $\mathrm{CH}_{2}$ ), 0.87 (t, $J=6.9 \mathrm{~Hz}$, $3 \mathrm{H}, \mathrm{CH}_{3}$ ). Calcd for $\mathrm{C}_{38} \mathrm{H}_{57} \mathrm{NO}_{7}: \mathrm{C}, 71.33 ; \mathrm{H}, 8.98 ; \mathrm{N}, 2.19$. Found: C, 71.30; H, 9.03; N, 2.24. 


\section{Theoretical Studies on the Structures of $(S)-2 a^{\mathrm{NO}}-\mathrm{OMe}$ and $(S)-2 b^{\mathrm{NO}}-\mathrm{OMe}$, and Their}

\section{Calculated CD Spectra}

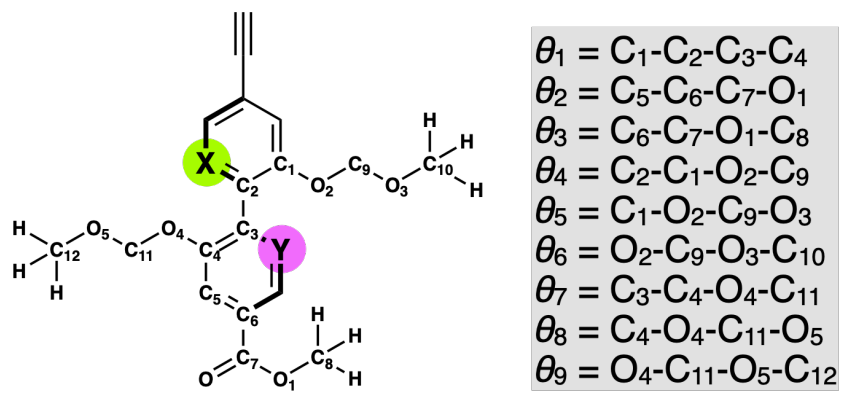

(S)-2a ${ }^{\text {NO-OMe }}\left(\mathbf{X}=\mathrm{N}^{+} \mathrm{O}^{-}, \mathbf{Y}=\mathrm{CH}\right)$

(S)-2b $\mathbf{b}^{\mathrm{NO}}$-OMe $\left(\mathbf{X}=\mathrm{CH}, \mathbf{Y}=\mathrm{N}+\mathrm{O}^{-}\right)$

The model compounds $\left((S)-\mathbf{2} \mathbf{a}^{\mathrm{NO}}-\mathrm{OMe}\right.$ and $\left.(S)-\mathbf{2} \mathbf{b}^{\mathbf{N O}}-\mathrm{OMe}\right)$ containing a methoxycarbonyl group instead of an icosyloxycarbonyl $\left(\mathrm{C}_{20} \mathrm{H}_{41} \mathrm{OCO}\right)$ group were used for the computational studies for simplicity. The initial structures of $(S)-\mathbf{2} \mathbf{a}^{\mathbf{N O}}-\mathrm{OMe}$ and $(S)-\mathbf{2} \mathbf{b}^{\mathbf{N O}}-\mathrm{OMe}$ were constructed on a Windows 10 PC with the GaussView 6 (Gaussian, Inc., Pittsburgh, PA). The conformational search algorithm CONFLEX (MMFF94s force field) was used to find their stable conformations by rotating nine selected dihedral angles $\left(\theta_{1}-\theta_{9}\right)$ in a stepwise manner in the CONFLEX software (Version 8A; CONFLEX, Tokyo, Japan). ${ }^{\text {S12-13 }}$ The resulting lower energy conformers, which differed from the most stable conformer by less than $20 \mathrm{kcal} / \mathrm{mol}$, were then fully optimized by the DFT calculations using the B3LYP functional with the 6-31G(d) basis set with GD3 ${ }^{\mathrm{S} 14}$ corrections for dispersion in Gaussian 16 software (Gaussian, Inc., Pittsburgh, PA). ${ }^{\text {S15 }}$ The theoretical CD spectra of $(S)-\mathbf{2 a}^{\text {NO }}$ OMe and $(S)-\mathbf{2} \mathbf{b}^{\text {NO}}$-OMe were calculated for each energy-minimized conformer with the Boltzmann populations of more than $1 \%$ based on the Gibbs free energy by the TD-DFT at CAM-B3LYP/6$311+\mathrm{G}(\mathrm{d}, \mathrm{p})$ level of theory with GD3 corrections for dispersion in Gaussian 16 software. The calculated rotational strengths in length gauge were expanded by the Gaussian functions and overlapped where the width of the band at the half-height was fixed at $0.30 \mathrm{eV}$. The calculated CD data were displayed as averaged spectra based on the Boltzmann populations of the corresponding energy-minimized conformers at $298.15 \mathrm{~K}$ (Figures S4 and S5). Computer resources for the DFT calculations were provided by the Information Technology Center of Nagoya University.

\section{Molecular Modeling of One-Handed Helical Cis-Transoidal Poly-(S)-2a ${ }^{\mathrm{NO}}$ and Poly-(R)-2b}

The molecular modeling and molecular mechanics (MM) calculations of the one-handed helical cis-transoidal poly- $(S)-\mathbf{2 a}^{\mathrm{NO}}$ and poly- $(R)-\mathbf{2 b}^{\mathrm{NO}}$ were conducted with the Universal force field ${ }^{\mathrm{S} 16-18}$ as implemented in the Materials Studio Modeling software (Version 8.0; Dassault Systèmes BIOVIA, San Diego, CA, USA) operated using a PC running under Windows 10 . The monomer unit structures of $(S)-\mathbf{2} \mathbf{a}^{\text {NO }}$ and $(R)-\mathbf{2} \mathbf{b}^{\text {NO }}$, in which the icosyloxycarbonyl $\left(\mathrm{C}_{20} \mathrm{H}_{41} \mathrm{OCO}\right)$ groups of the pendants were 
replaced by the methoxycarbonyl groups for simplicity, were constructed using the crystal structure of an analogous biphenylylacetylene monomer ${ }^{\mathrm{S} 19}$ and the geometries were optimized by the density functional theory (DFT) at the B3LYP/6-31G(d) level using the Gaussian 16 package (Gaussian, Inc., Pittsburgh, PA). ${ }^{\mathrm{S} 15}$ Because the relationships between the macromolecular helicity (right- or lefthanded helix) and axial chirality of the 2-arylpyridyl- $N$-oxide pendants $((S)$ - or $(R))$ of $c i s$-poly- $(S)$ $\mathbf{2 a}^{\text {NO }}$ and $c i s-p o l y-(R)-\mathbf{2 b}^{\text {NO }}$ are not presently elucidated, the main-chain helical senses of cistransoidal poly- $(S)-\mathbf{2} \mathbf{a}^{\text {NO }}$ and poly- $(R)-\mathbf{2} \mathbf{b}^{\text {NO }}$ are tentatively assumed to be right- and left-handed helices, respectively. The polymer models (30 repeating monomer units) of right- and left-handed helical cis-transoidal poly- $(S)-\mathbf{2} \mathbf{a}^{\mathrm{NO}}$ and poly- $(R)-\mathbf{2} \mathbf{b}^{\mathbf{N O}}$, respectively, were then constructed by a Polymer Builder module in the Materials Studio Modeling software.

The main-chain geometrical parameters, such as the bond lengths, the bond angles, and the internal rotation angles, were defined as a 13 unit/5 turn (13/5) helix on the basis of the helical structure of a poly(biphenylylacetylene) (PBPA) composed of achiral monomer units (poly-1b in Figure 1a) with a monomer unit height of $2.34 \AA$ postulated by X-ray diffraction (XRD) analysis of the oriented film. ${ }^{\mathrm{S} 20}$ The geometry optimizations were carried out without any cutoff by the smart minimizer in three steps while maintaining the 13/5 helical structures. First, the starting conformations were subject to the steepest decent optimization to eliminate the worst steric conflicts. Second, subsequent optimization until the convergence using a conjugate gradient algorithm was performed. The optimized polymer models were obtained by the further energy minimization using the Newton method with the $0.1 \mathrm{kcal} \cdot \mathrm{mol}^{-1} \cdot \AA^{-1}$ convergence criterion (Figure S25).

\section{Macromolecular Helicity and Axial Chirality Induction in Optically Inactive Poly-rac-2a ${ }^{\text {NO }}$ with Enantiomeric Alcohols and Subsequent Dual Static Memory Mediated by Deracemization of the Axially Chiral Biaryl Pendants}

The concentration of poly-rac-2 $\mathbf{a}^{\mathrm{NO}}$ was calculated based on the monomer units and was $1.0 \mathrm{mM}$ unless otherwise stated. A typical experimental procedure for "the macromolecular helicity and axial

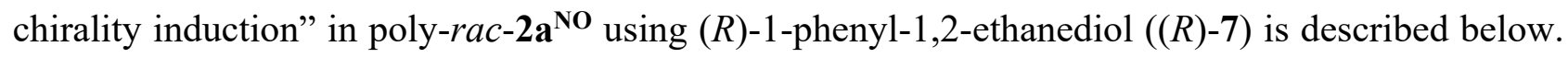
Stock solutions of poly-rac-2a $\mathbf{a}^{\text {NO }}(1.0 \mathrm{mM})$ and $(R)-7(100 \mathrm{mM})$ in toluene were prepared in 5-mL flasks equipped with stopcocks. $500 \mu \mathrm{L}$ aliquots of the poly-rac-2a ${ }^{\text {NO }}$ solution were transferred to five vessels equipped with a screwcap using a Hamilton microsyringe. After the solvents were removed by evaporation, an appropriate amount of the stock solution of $(R)-7$ was added to each vessel and the solutions were diluted with toluene to keep the poly-rac-2 $\mathbf{a}^{\mathbf{N O}}$ concentration at $1.0 \mathrm{mM}$ $\left([7] /\left[\right.\right.$ poly-rac-2a $\left.\mathbf{a}^{\mathrm{NO}}\right]=1,5,20,50$, and 100), then the solutions were immediately mixed with a vibrator (IKA, Japan). After the solutions were allowed to stand at 25 or $40{ }^{\circ} \mathrm{C}$, the absorption and $\mathrm{CD}$ spectra were recorded at $25^{\circ} \mathrm{C}$ at appropriate time intervals until no further increase in the CD 
intensity was observed (Figures 2, 3, and S17). In the same way, "the macromolecular helicity and axial chirality induction" experiments were performed with different chiral alcohols $((S)-\mathbf{3},(S)-\mathbf{4},(R)$ 5, $(R, R)-6$, and $(S)-7$; see Figure $3 \mathrm{a})$ in toluene at $40{ }^{\circ} \mathrm{C}\left(\left[\right.\right.$ chiral alcohol] $/\left[\right.$ poly-rac-2a $\left.{ }^{\mathrm{NO}}\right]=20$ or 50 ; Figures 2 and 3$)$.

Among the chiral alcohols tested, $(R)$ - or $(S)$-7 was superior to other chiral alcohols (Figure 3), inducing the maximum CD intensity in poly-rac-2a ${ }^{\mathrm{NO}}$ when $(R)-7$ ( $\geq 50$ equiv.) was used (Figure S17). Thus, the subsequent "dual static memory of the helicity and axial chirality" experiments were

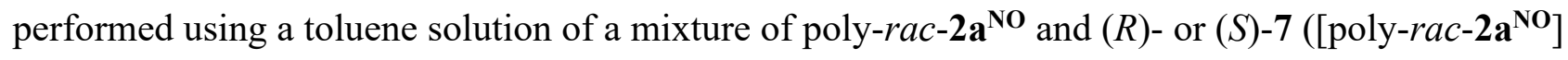
$=1.0 \mathrm{mM},[7] /\left[\right.$ poly-rac-2a $\left.{ }^{\mathrm{NO}}\right]=50$ ), which was then allowed to stand at $40{ }^{\circ} \mathrm{C}$ for seven days. During the annealing process, the dynamically racemic biaryl pendants of poly-rac-2a ${ }^{\text {NO }}$ most likely deracemized in an enantioselective fashion, assisted by interaction with $(R)$ - or $(S)$-7 (Figure 1b), thus inducing an excess of one-handed helical sense in the poly-2a ${ }^{\mathbf{N O}}$ backbone, which can be subsequently memorized after removing the chiral alcohol, thus producing an optically active $h$-polyderac-2a $\mathbf{a}^{\mathbf{N O}}$. A typical experimental procedure for isolating $h$-poly-derac-2-2 $\mathbf{a}^{\mathbf{N O}}$ is described below.

After removing the solvent by evaporation, followed by washing with methanol, the residue was diluted with toluene at room temperature and the solution was poured into a large amount of methanol at $-10{ }^{\circ} \mathrm{C}$ to remove $(R)-7$. The precipitated polymer was then collected by centrifugation, washed with methanol, and then dried in vacuo at room temperature overnight. Complete removal of $(R)-7$ was confirmed by ${ }^{1} \mathrm{H}$ NMR measurement of the isolated polymer (Figure S18). After the isolated polymer with the dual static memory of the helicity and axial chirality was dissolved in toluene at $10{ }^{\circ} \mathrm{C}$, the absorption and $\mathrm{CD}$ spectra were recorded at -10 and $25^{\circ} \mathrm{C}$ at appropriate time intervals (Figures 2, S13e,f, and S22). As described in the main text, the helicity-memorized poly-rac-2a ${ }^{\text {NO }}$ induced by $(R)$ - and $(S)-7$ through deracemization of the dynamically racemic biaryl pendants are

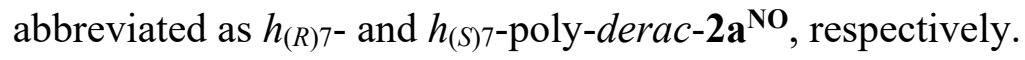

\section{Asymmetric Allylation of Benzaldehyde with Allyltrichlorosilane Catalyzed by Helical Polymers}

A typical procedure for the asymmetric allylation using poly-(S)-2 $\mathbf{a}^{\mathbf{N O}}$ as a helical polymer-based asymmetric catalyst is described as follows. Poly- $(S)-\mathbf{2} \mathbf{a}^{\text {NO }}$ (3 $\mu$ mol based on the pyridine $N$-oxide residue of poly- $\left.(S)-\mathbf{2} \mathbf{a}^{\mathbf{N O}}\right)$ was dissolved in toluene $(70 \mu \mathrm{L})$ under an argon atmosphere. To this was added benzaldehyde (8) (3.3 $\mathrm{mg}, 0.031 \mathrm{mmol})$ dissolved in toluene (50 $\mu \mathrm{L}), N$-ethyldiisopropylamine ( $\left.{ }^{i} \operatorname{Pr}_{2} \mathrm{NEt}\right)(17 \mu \mathrm{L}, 0.099 \mathrm{mmol})$, allyltrichlorosilane $(8.2 \mathrm{mg}, 0.047 \mathrm{mmol})$ dissolved in toluene (50 $\mu \mathrm{L})$, and 1,1,2,2-tetrachloroethane $(2.5 \mathrm{mg}, 0.015 \mathrm{mmol})$ dissolved in toluene $(25 \mu \mathrm{L})$ at $-10{ }^{\circ} \mathrm{C}$. The mixture was stirred at $-10^{\circ} \mathrm{C}$ for $3 \mathrm{~h}$. After the addition of aqueous $\mathrm{NaOH}(1.0 \mathrm{M}, 0.20 \mathrm{~mL})$ to quench the reaction, chloroform was added, and the organic layer was washed with brine and dried over 
anhydrous $\mathrm{MgSO}_{4}$. After filtration, the solvent was evaporated to dryness in vacuo. The conversion of 8 to the allyl alcohol 9 was determined to be $>99 \%$ by ${ }^{1} \mathrm{H}$ NMR analysis using 1,1,2,2tetrachloroethane as the internal standard. After the residue was passed through a short pad of silica gel using chloroform as the eluent, the enantiomeric excess (ee) value of the product 9 ( $96 \%$ ee, $R$ rich) was determined by chiral HPLC analysis using a CHIRALCEL OD-H column ( $n$-hexane-2propanol $(98 / 2, \mathrm{v} / \mathrm{v})$; flow rate $0.8 \mathrm{~mL} / \mathrm{min}$; temperature ca. $20{ }^{\circ} \mathrm{C}: t_{\mathrm{r}}=18.4 \mathrm{~min}$ (for $R$-isomer), $t_{\mathrm{r}}=$ $20.8 \mathrm{~min}$ (for $S$-isomer) (see Figure S23). ${ }^{\mathrm{S} 21}$ Allylation reactions of 8 with allyltrichlorosilane using optically-active and -inactive $\mathbf{2} \mathbf{a}^{\mathrm{NO}}$-based monomer and polymers as catalysts were also performed in a similar manner and the results are summarized in Table 3. 


\section{Supporting Data}
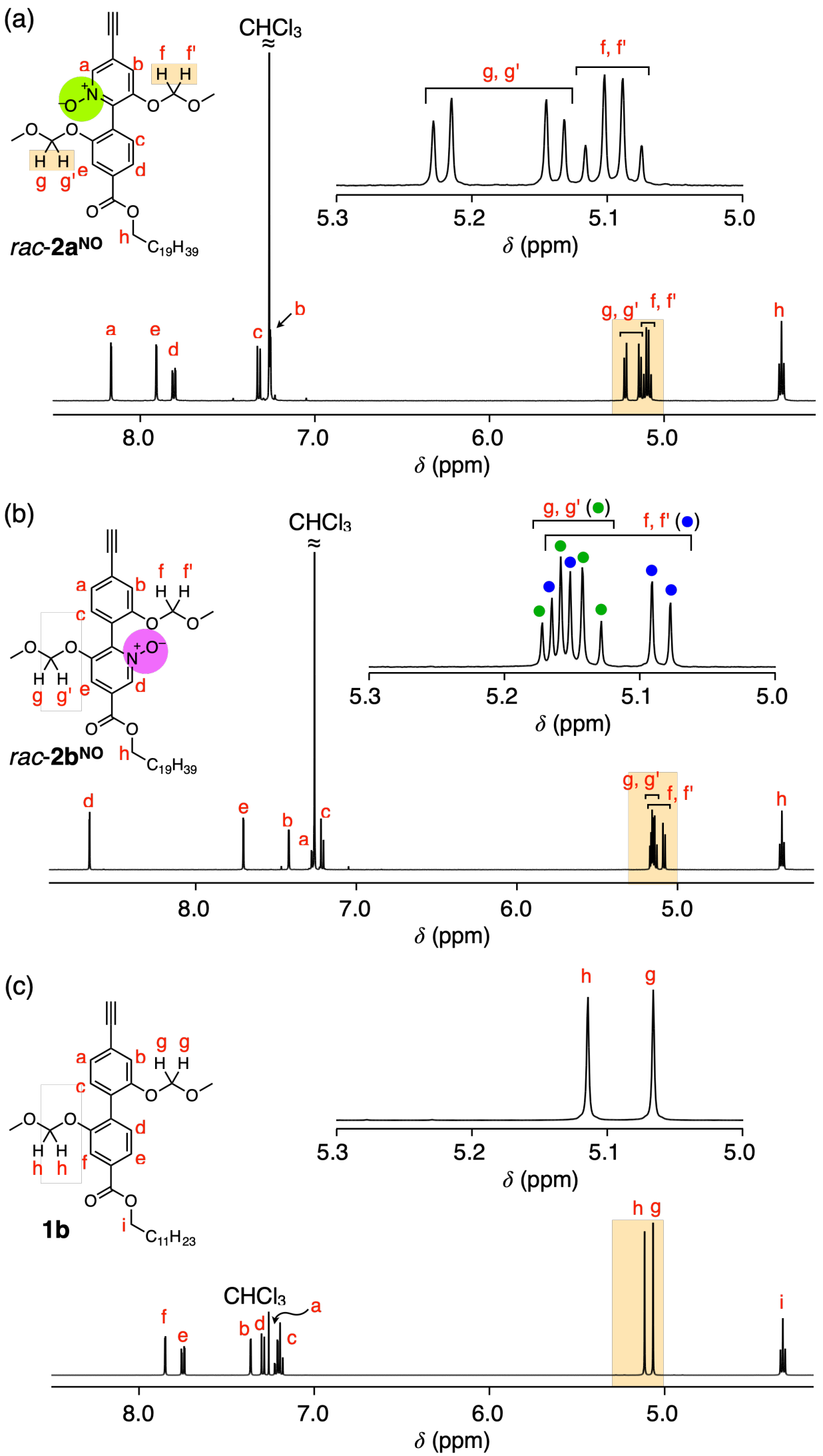

Figure S1. Partial ${ }^{1} \mathrm{H}$ NMR $\left(500 \mathrm{MHz}, \mathrm{CDCl}_{3}, 25^{\circ} \mathrm{C}\right)$ spectra of $r a c-2 \mathbf{a}^{\mathrm{NO}}(\mathrm{a}), \operatorname{rac}-\mathbf{2} \mathbf{b}^{\mathbf{N O}}(\mathrm{b})$, and $\mathbf{1 b}$ (c). For the signal assignments, see Figures S27-S29, respectively. 

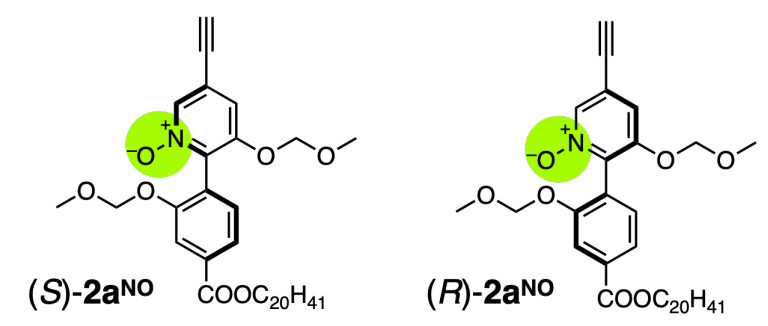

(a)

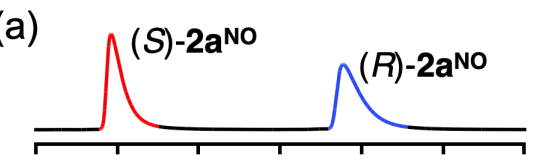

(b)

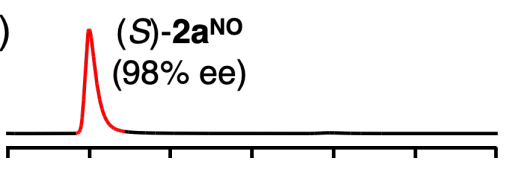

(c)

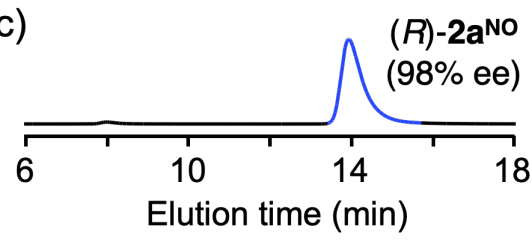

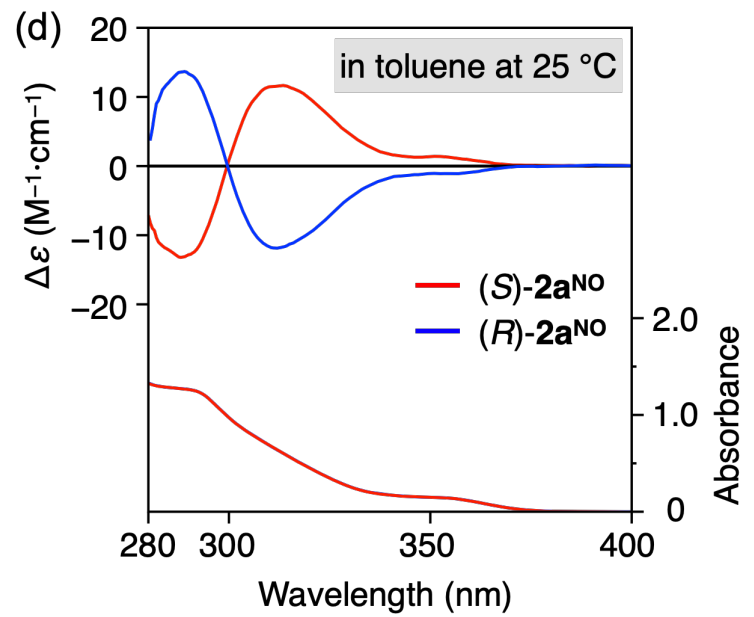

Figure S2. (a-c) Chromatograms for the resolution of $\mathbf{r a c}-\mathbf{2} \mathbf{a}^{\mathbf{N O}}(\mathrm{a})$ and the fractionated $(S)-\mathbf{2} \mathbf{a}^{\mathbf{N O}}$ (b) and $(R)-\mathbf{2} \mathbf{a}^{\mathrm{NO}}(\mathrm{c})$. Chromatographic conditions: column, CHIRALPAK IB (0.46 cm (i.d.) $\left.\times 25 \mathrm{~cm}\right)$; eluent, $n$-hexane/chloroform/2-propanol (70/30/2, v/v/v); flow rate, $0.80 \mathrm{~mL} / \mathrm{min}$; temperature, $0{ }^{\circ} \mathrm{C}$. (d) $\mathrm{CD}$ and absorption spectra of $(S)$ - and $(R)-\mathbf{2} \mathbf{a}^{\mathrm{NO}}$ in toluene at $25^{\circ} \mathrm{C}$. $\left[\mathbf{2 \mathbf { a } ^ { \mathrm { NO } }}\right]=1.0 \times 10^{-3} \mathrm{M}$. The absolute configurations were assigned based on the TD-DFT calculations (Figure S4). 

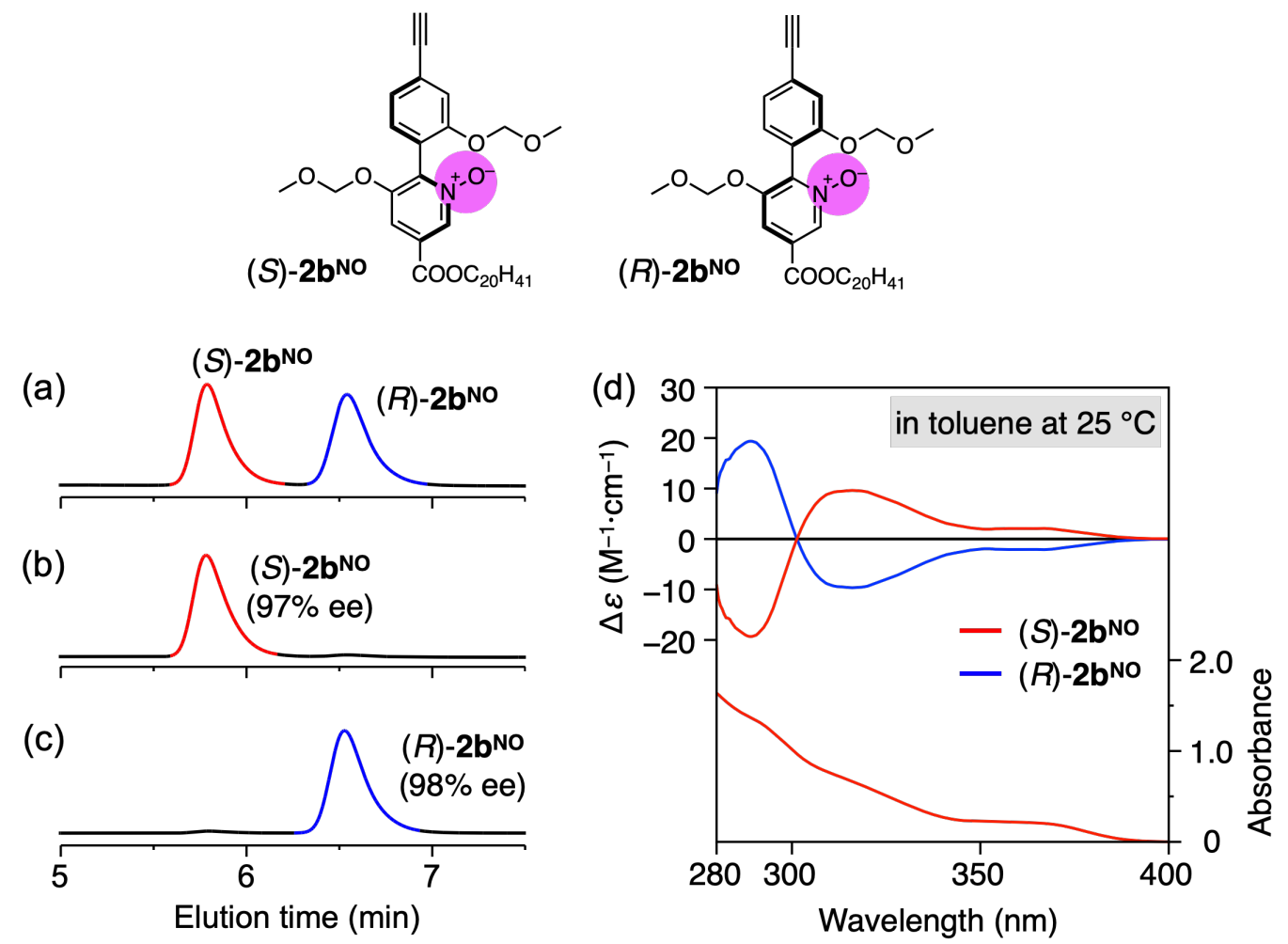

Figure S3. (a-c) Chromatograms for the resolution of $r a c-\mathbf{2 b}^{\text {NO }}$ (a) and the fractionated $(S)-\mathbf{2} \mathbf{b}^{\text {NO }}$ (b) and $(R)-2 \mathbf{b}^{\text {NO }}(\mathrm{c})$. Chromatographic conditions: column, CHIRALPAK IB (0.46 cm (i.d.) $\left.\times 25 \mathrm{~cm}\right)$; eluent, $n$-hexane/chloroform/2-propanol (70/30/2, v/v/v); flow rate, $0.80 \mathrm{~mL} / \mathrm{min}$; temperature, $0{ }^{\circ} \mathrm{C}$. (d) $\mathrm{CD}$ and absorption spectra of $(S)$ - and $(R)-\mathbf{2} \mathbf{b}^{\mathrm{NO}}$ in toluene at $25^{\circ} \mathrm{C}$. $\left[\mathbf{2 \mathbf { b } ^ { \mathrm { NO } }}\right]=1.0 \times 10^{-3} \mathrm{M}$. The absolute configurations were assigned based on the TD-DFT calculations (Figure S5). 


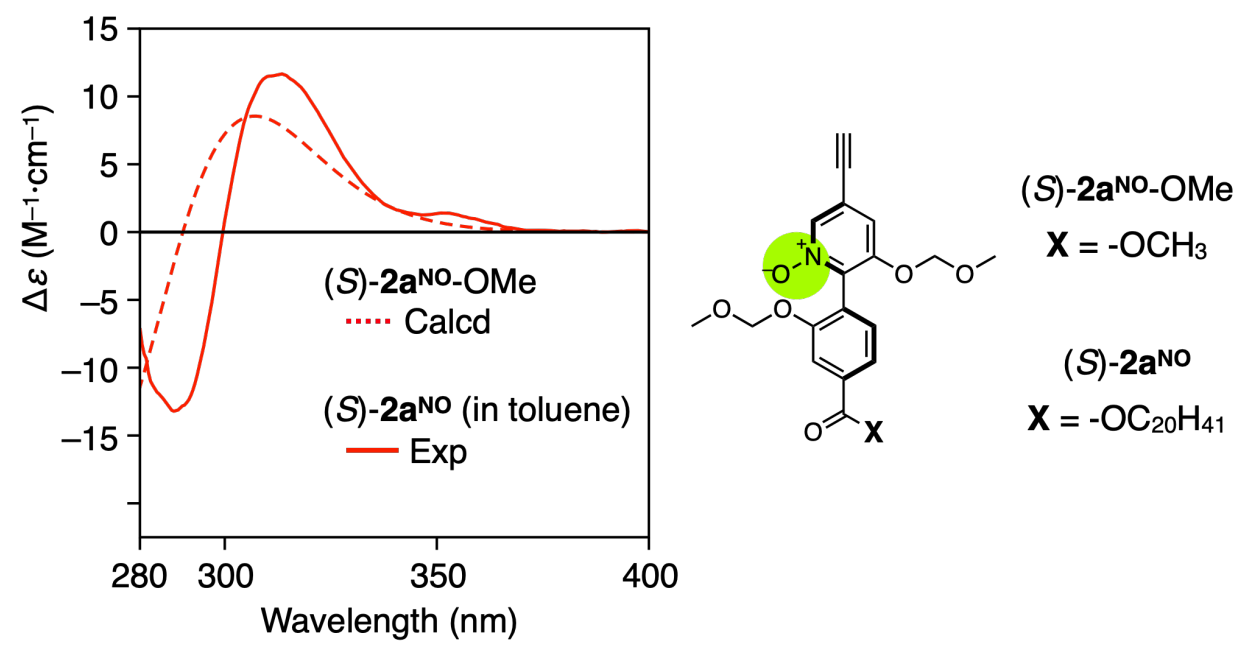

Figure S4. Observed (Figure S2d, red solid line) and calculated (red dotted line) CD spectra of $(S)$ $\mathbf{2 a}^{\text {NO }}\left(1.0 \times 10^{-3} \mathrm{M}\right)$ and $(S)-\mathbf{2} \mathbf{a}^{\text {NO }}-\mathrm{OMe}$, respectively. The calculated CD spectrum of $(S)-\mathbf{2} \mathbf{a}^{\mathrm{NO}}-\mathrm{OMe}$ was obtained by TD-DFT at the CAM-B3LYP/6-311+G $(d, p)$ level with GD3 corrections for dispersion.

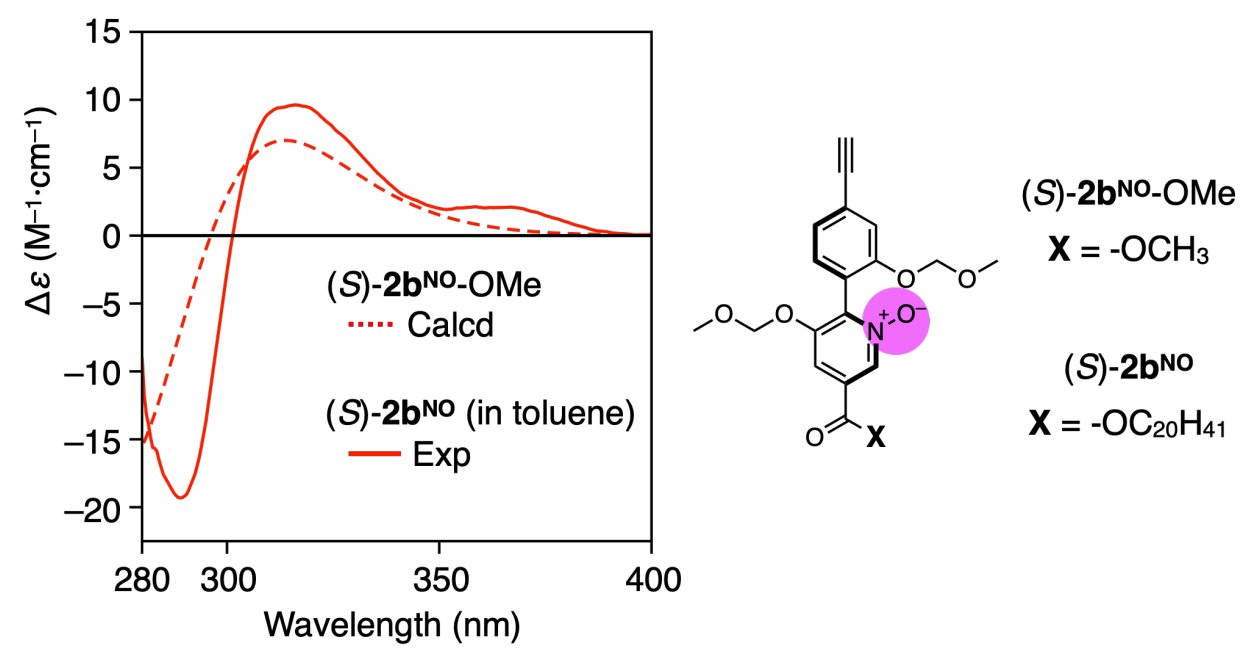

Figure S5. Observed (Figure S3d, red solid line) and calculated (red dotted line) CD spectra of $(S)$ $\mathbf{2 b}^{\text {NO }}\left(1.0 \times 10^{-3} \mathrm{M}\right)$ and $(S)-\mathbf{2} \mathbf{b}^{\text {NO }}-$ OMe, respectively. The calculated CD spectrum of $(S)-\mathbf{2} \mathbf{b}^{\text {NO_-OMe }}$ was obtained by TD-DFT at the CAM-B3LYP/6-311+G (d,p) level with GD3 corrections for dispersion. 


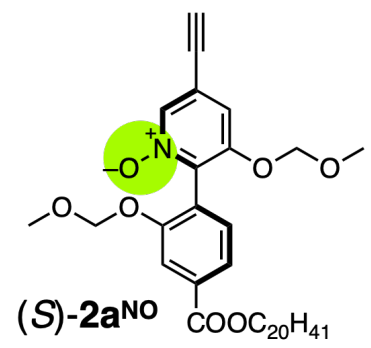

(a)

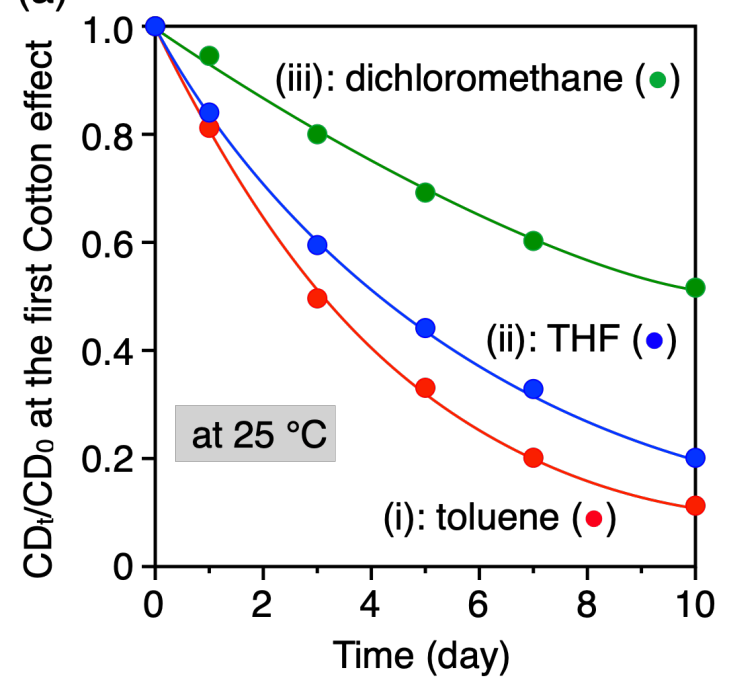

(c)

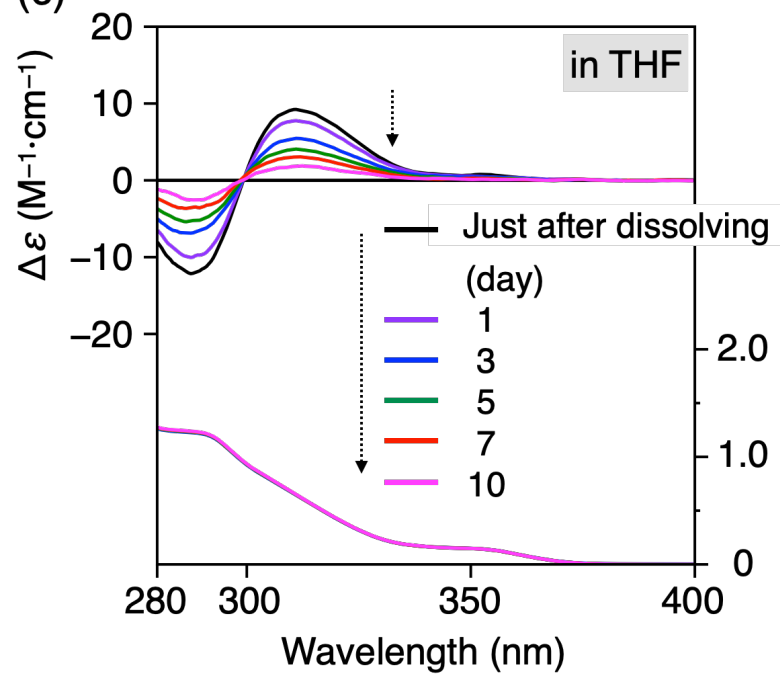

(b)

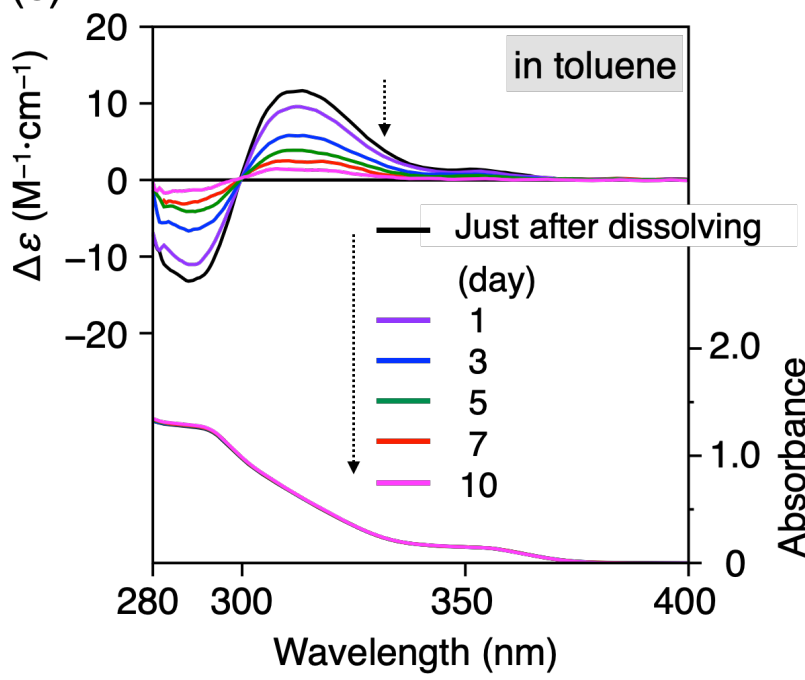

(d)

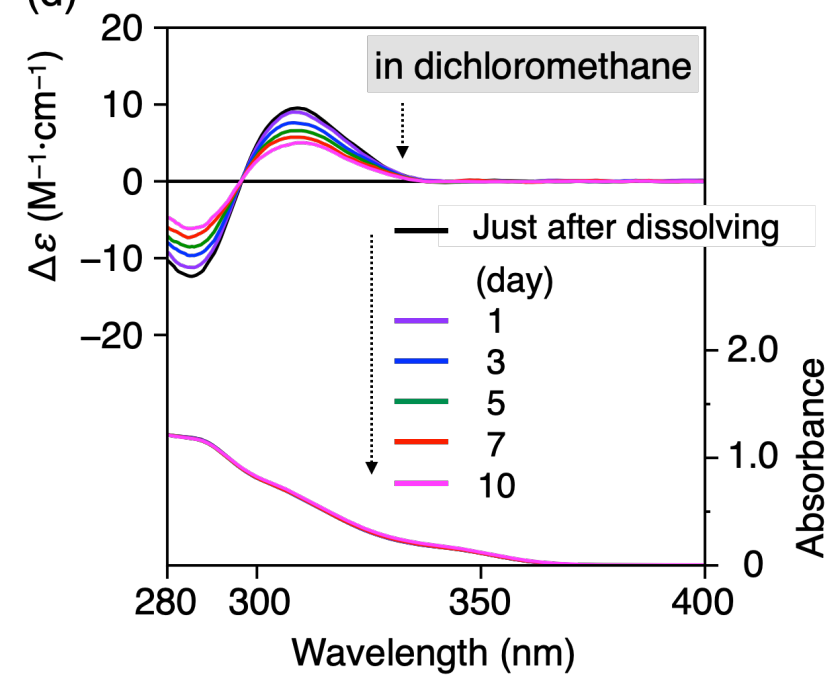

Figure S6. (a) Plots of the time-dependent $C D$ intensity changes at the first Cotton effect $\left(\mathrm{CD}_{t} / \mathrm{CD}_{0}\right)$ of (S)-2a ${ }^{\text {NO }}$ measured in toluene (i), THF (ii), and dichloromethane (iii) at $25{ }^{\circ} \mathrm{C}$. $\mathrm{CD}_{0}$ represents the initial CD intensity of $(S)-\mathbf{2} \mathbf{a}^{\text {NO }}\left(98 \%\right.$ ee) in each solvent at $25{ }^{\circ} \mathrm{C}$. The corresponding time-dependent $\mathrm{CD}$ and absorption spectral changes at $25^{\circ} \mathrm{C}$ are also shown in $(\mathrm{b}-\mathrm{d}) .\left[\mathbf{2} \mathbf{a}^{\mathrm{NO}}\right]=1.0 \mathrm{mM}$. 


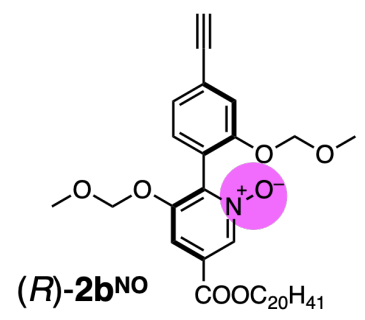

(a)

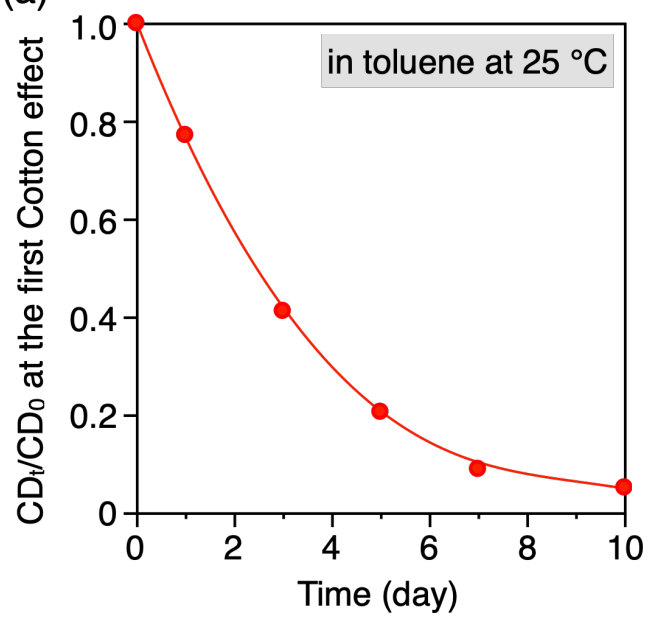

(b)

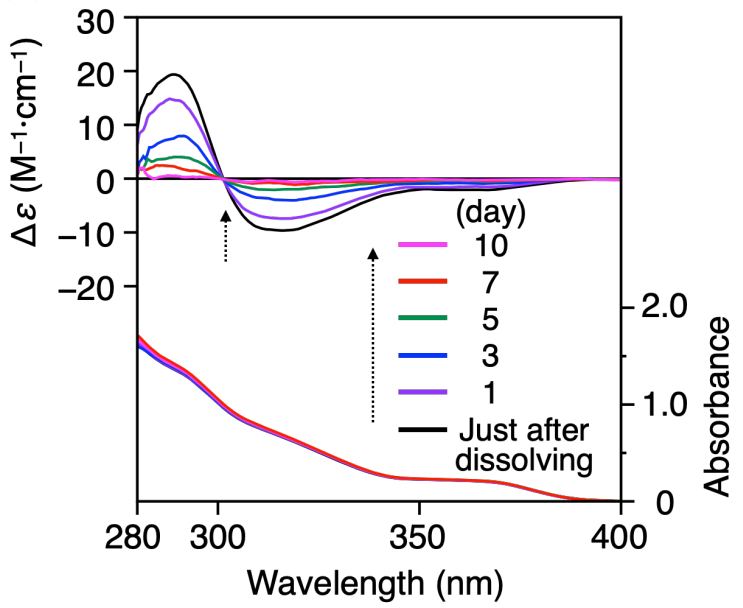

Figure S7. (a) Plots of the time-dependent CD intensity changes at the first Cotton effect $\left(\mathrm{CD}_{t} / \mathrm{CD}_{0}\right)$ of $(R)$-2 $\mathbf{b}^{\mathrm{NO}}$ measured in toluene at $25^{\circ} \mathrm{C} . \mathrm{CD}_{0}$ represents the initial CD intensity of $(R)$-2 $\mathbf{2} \mathbf{b}^{\mathrm{NO}}(98 \%$ ee) in toluene at $25{ }^{\circ} \mathrm{C}$. The corresponding time-dependent $\mathrm{CD}$ and absorption spectral changes at $25^{\circ} \mathrm{C}$ are also shown in (b). $\left[2 \mathbf{b}^{\mathrm{NO}}\right]=1.0 \mathrm{mM}$. 

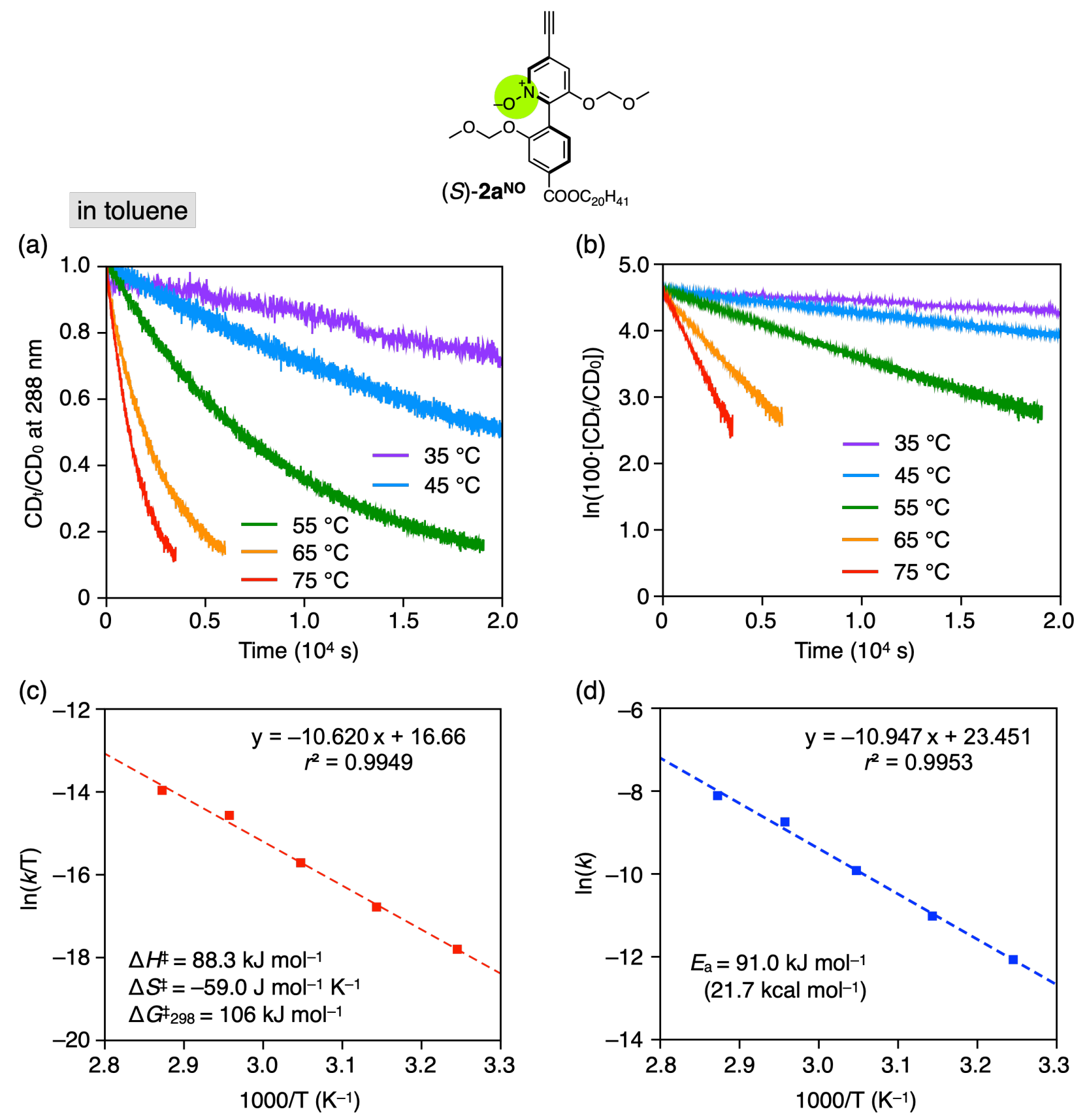

Figure S8. $(\mathrm{a}, \mathrm{b})$ Plots of the time-dependent CD intensity changes at $288 \mathrm{~nm}$ of $(S)-\mathbf{2} \mathbf{a}^{\mathbf{N O}}$ in toluene at $35,45,55,65$, and $75^{\circ} \mathrm{C}$. (c) Eyring and (d) Arrhenius plots for the racemization of $(S)-\mathbf{2 a} \mathbf{a}^{\text {NO }}$ in toluene. 

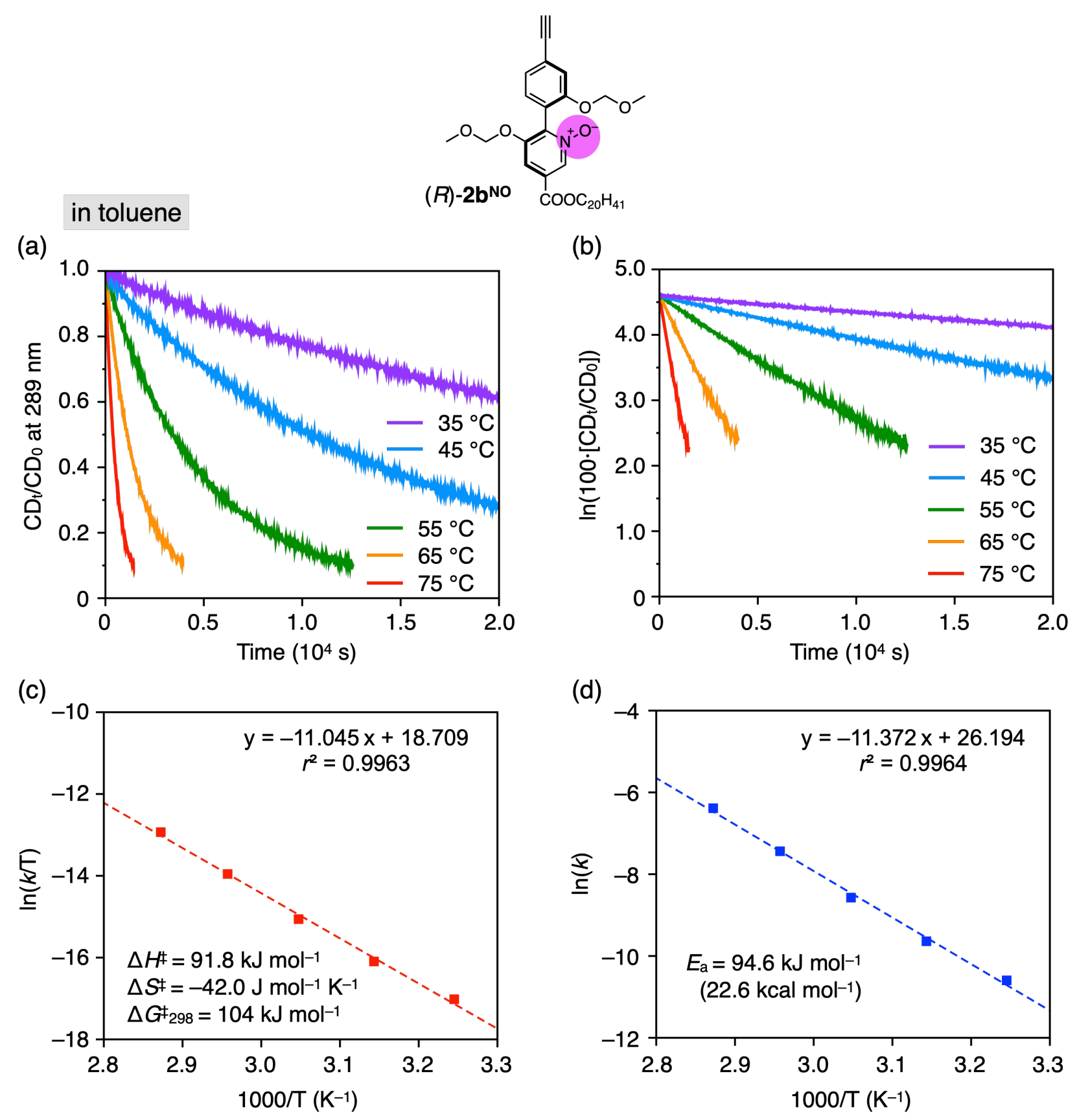

Figure S9. $(\mathrm{a}, \mathrm{b})$ Plots of the time-dependent CD intensity changes at $289 \mathrm{~nm}$ of $(R)-\mathbf{2} \mathbf{b}^{\mathrm{NO}}$ in toluene at $35,45,55,65$, and $75^{\circ} \mathrm{C}$. (c) Eyring and (d) Arrhenius plots for the racemization of $(R)-\mathbf{2} \mathbf{b}^{\text {NO }}$ in toluene. 


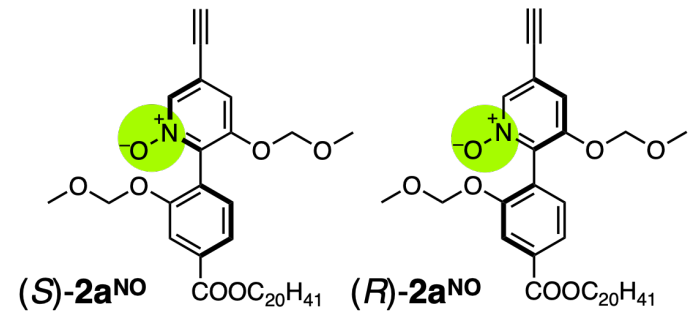

(a)

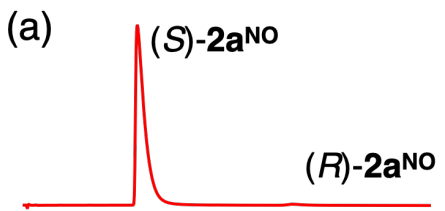

$98 \%$ ee

(entry 2 in Table 1)

Standing in toluene containing a mixture of 4-propoxyphenylboronic acid, diphenylacetylene, $\mathrm{PPh}_{3}$, and $\mathrm{KOH}$ at $0{ }^{\circ} \mathrm{C}$ for $24 \mathrm{~h}$

$97 \%$ ee

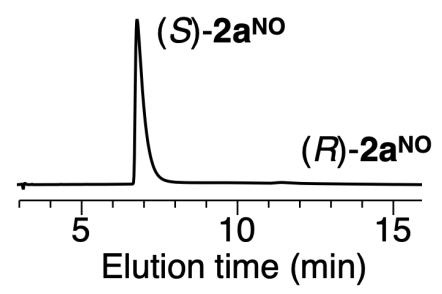

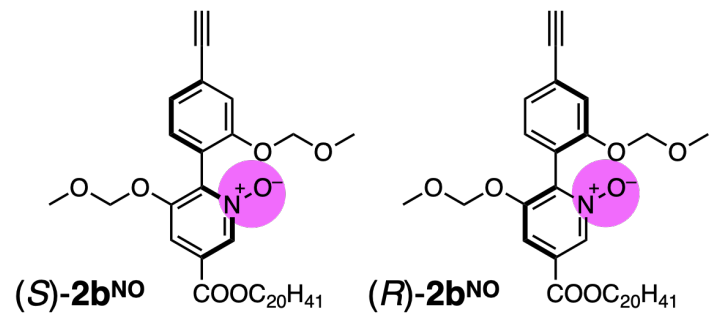

(b)

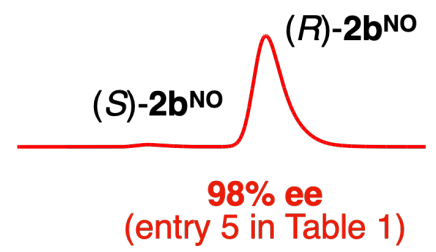

Standing in toluene containing a mixture of 4-propoxyphenylboronic acid, diphenylacetylene, $\mathrm{PPh}_{3}$, and $\mathrm{KOH}$ at $0{ }^{\circ} \mathrm{C}$ for $24 \mathrm{~h}$

$97 \%$ ee

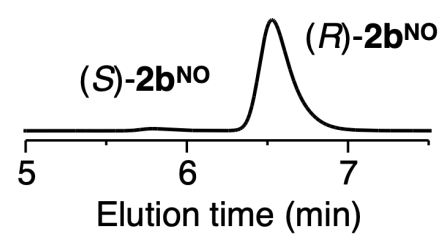

[4-propoxyphenylboronic acid]/[diphenylacetylene]/[PPh 3$] /[\mathrm{KOH}]=3 / 8 / 6 / 5$

Figure S10. Chromatograms for the resolution of the $(S)-\mathbf{2} \mathbf{a}^{\mathbf{N O}}$ (a; entry 2 in Table 1$)$ and $(R)-\mathbf{2} \mathbf{b}^{\mathrm{NO}}$ (b; entry 5 in Table 1) feed monomers with $98 \%$ ee before (upper) and after (bottom) allowing to stand under the polymerization conditions (see Section 2) except for the absence of a rhodium catalyst $\left([\mathrm{Rh}(\mathrm{nbd}) \mathrm{Cl}]_{2}\right)$, that is, in toluene containing a mixture of 4-propoxyphenylboronic acid, diphenylacetylene, $\mathrm{PPh}_{3}$, and $\mathrm{KOH}$ at $0{ }^{\circ} \mathrm{C}$ for $24 \mathrm{~h}\left(\left[\mathbf{2}^{\mathrm{NO}}\right]=0.125 \mathrm{M},\left[\mathbf{2}^{\mathrm{NO}}\right] /[4\right.$-propoxyphenylboronic acid] $/[$ diphenylacetylene]/[PPh 3$] /[\mathrm{KOH}]=1250 / 3 / 8 / 6 / 5)$, followed by evaporating the solvent. Chromatographic conditions: column, CHIRALPAK IB (0.46 cm (i.d.) $\times 25 \mathrm{~cm})$; eluent, $n$ hexane/chloroform/2-propanol (70/30/2, v/v/v); flow rate, $1.0 \mathrm{~mL} / \mathrm{min}$; temperature, $0{ }^{\circ} \mathrm{C}$. 
<smiles>C#Cc1cc(OCOC)c(-c2ccc(C=C)cc2OCOC)c(OCOC)c1</smiles>

(a) Entry 1 in Table 2

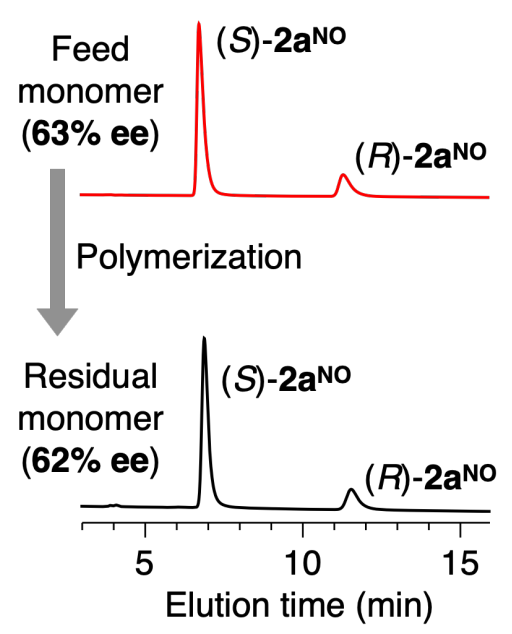

(b) Entry 2 in Table 2

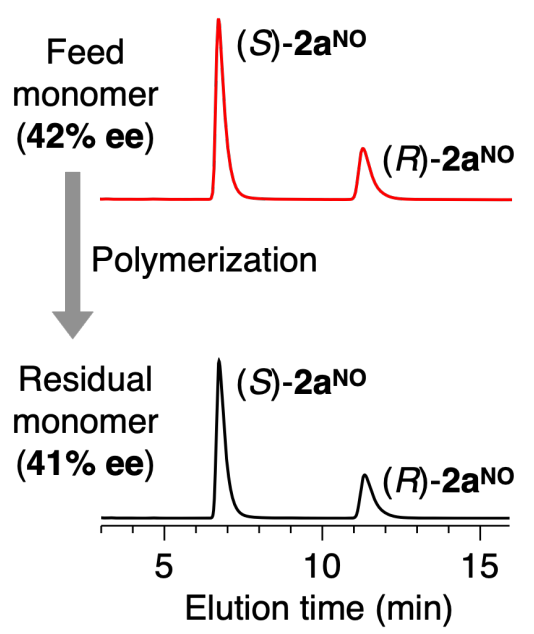

(c) Entry 3 in Table 2
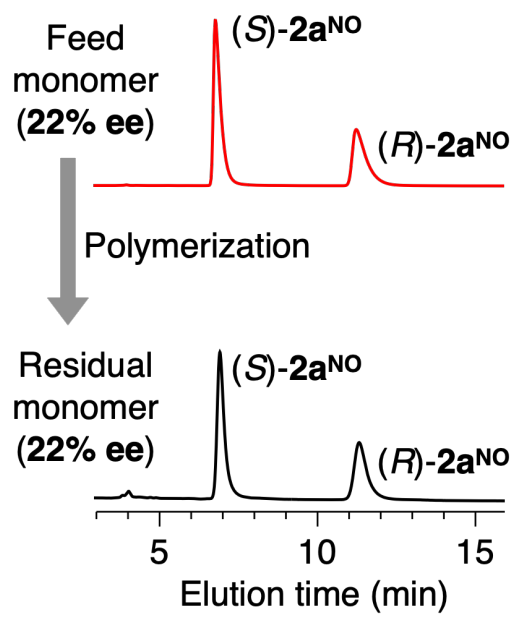

Figure S11. Chromatograms for the resolution of the nonracemic $\mathbf{2 a}^{\mathrm{NO}}$ ( $S$-rich) feed monomers used for polymerizations; entries 1 (a; 63\% ee), 2 (b; 42\% ee), and 3 (c; 22\% ee) in Table 2 (upper) and the residual monomers after polymerizations (bottom) (for details of the polymerization conditions, see Section 2). Chromatographic conditions: column, CHIRALPAK IB (0.46 cm (i.d.) $\times 25 \mathrm{~cm})$; eluent, $n$-hexane/chloroform/2-propanol $(70 / 30 / 2, \mathrm{v} / \mathrm{v} / \mathrm{v})$; flow rate, $1.0 \mathrm{~mL} / \mathrm{min}$; temperature, $0{ }^{\circ} \mathrm{C}$. 


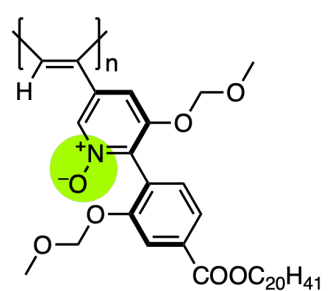

(i): poly-(R)-2anO (—)

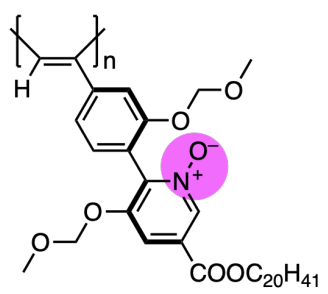

(ii): poly-(R)-2bNO (- - - )

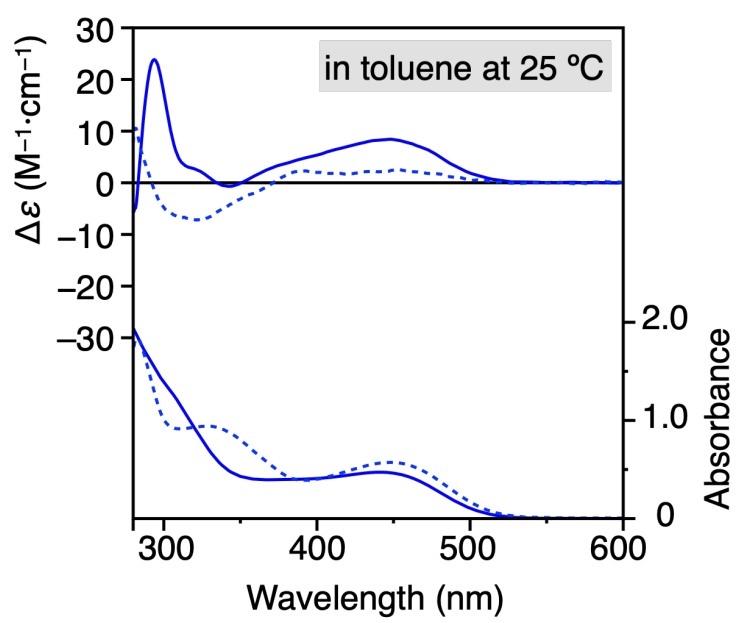

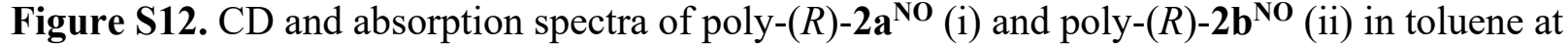
$25{ }^{\circ} \mathrm{C}$. $[$ Polymer $]=1.0 \mathrm{mM}$. 
(a)

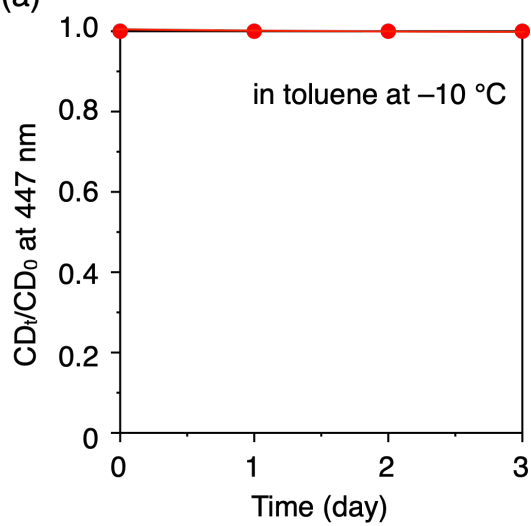

(c)

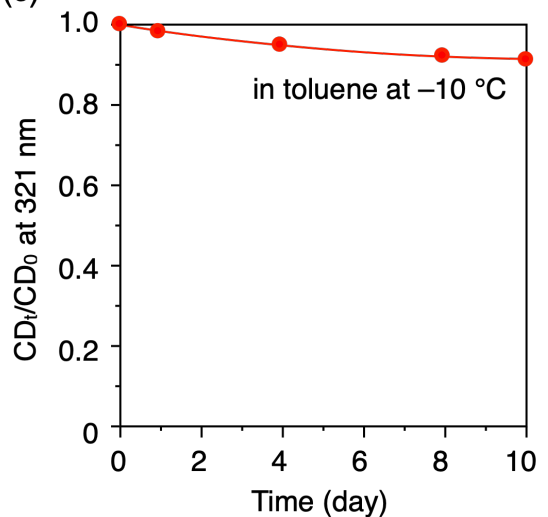

(e)

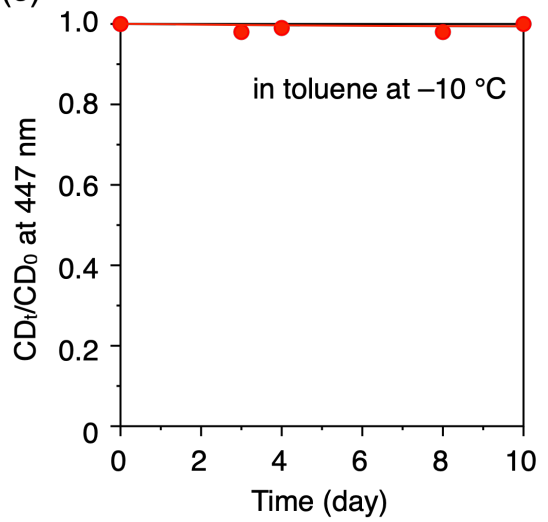

(b)

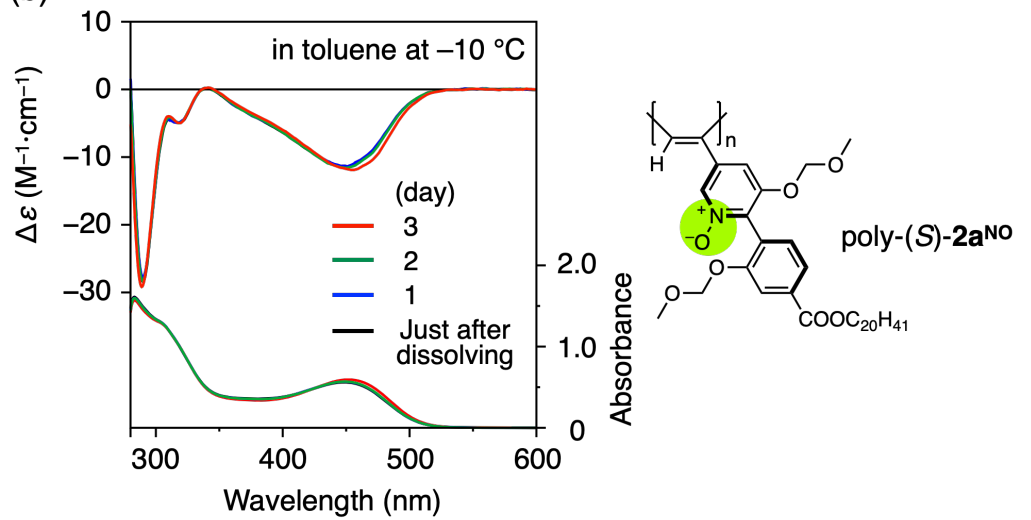

(d)

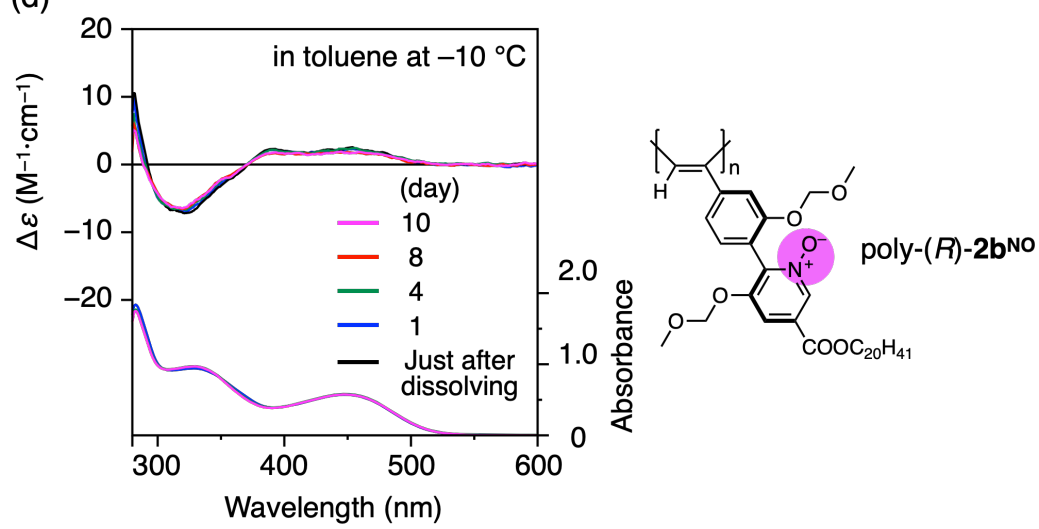

(f)

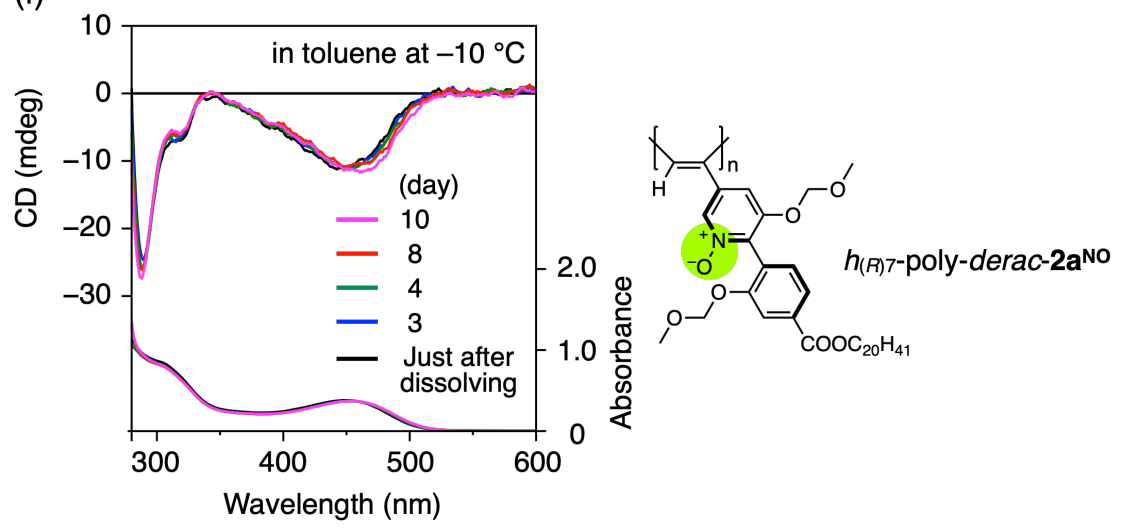

Figure S13. (a,c,e) Plots of the time-dependent $C D$ intensity changes $\left(\mathrm{CD}_{t} / \mathrm{CD}_{0}\right)$ at $447(\mathrm{a}, \mathrm{c})$ and 321 (b) nm of poly- $(S)-\mathbf{2} \mathbf{a}^{\mathrm{NO}}(\mathrm{a})$, poly- $(R)-\mathbf{2} \mathbf{b}^{\mathrm{NO}}(\mathrm{b})$, and the helicity-memorized $h_{(R) 7-\text { poly-derac-2a }} \mathbf{a}^{\mathrm{NO}}(\mathrm{c})$ measured in toluene at $-10{ }^{\circ} \mathrm{C}$ after storage at $-10{ }^{\circ} \mathrm{C}$. $\mathrm{CD}_{0}$ represents the initial $\mathrm{CD}$ intensity of each polymer in toluene at $-10^{\circ} \mathrm{C}$. The corresponding time-dependent $\mathrm{CD}$ and absorption spectral changes at $-10{ }^{\circ} \mathrm{C}$ are also shown in $(\mathrm{b}, \mathrm{d}, \mathrm{f})$. [Polymer $]=1.0 \mathrm{mM}$. 


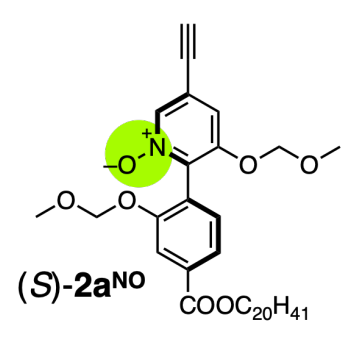

(i): toluene $(O)$

(ii): $\operatorname{THF}(\mathrm{O})$

(iii): dichloromethane $(\mathrm{O})$

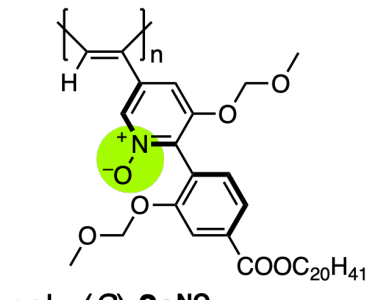

poly-(S)-2a No

(iv): toluene (๑)

(v): THF (๑)

(vi): dichloromethane (๑)

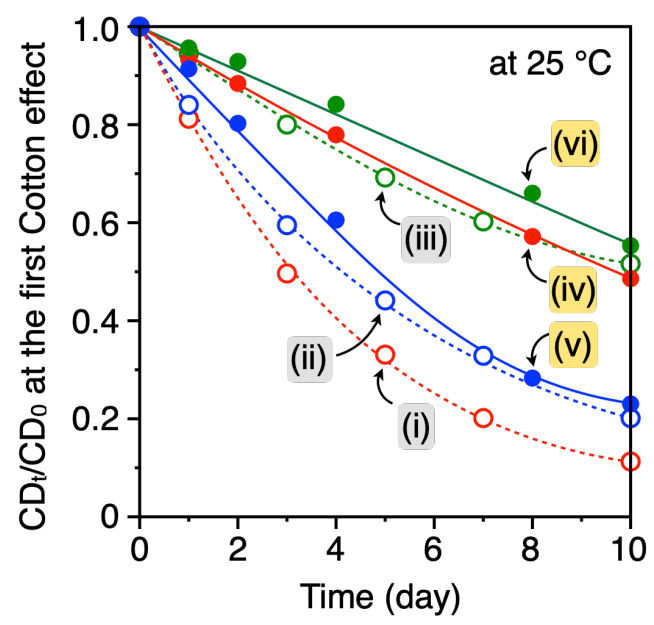

Figure S14. Plots of the time-dependent $C D$ intensity changes at the first Cotton effect $\left(\mathrm{CD}_{t} / \mathrm{CD}_{0}\right)$ of $(S)-\mathbf{2} \mathbf{a}^{\text {NO }}$ (i-iii) and poly-(S)-2a $\mathbf{a}^{\text {NO }}$ (iv-vi) measured in toluene (i and iv), THF (ii and v), and dichloromethane (iii and vi) at $25{ }^{\circ} \mathrm{C}$. $\mathrm{CD}_{0}$ represents the initial $\mathrm{CD}$ intensity of $(S)-\mathbf{2} \mathbf{a}^{\mathrm{NO}}$ (98\% ee) and poly- $(S)-\mathbf{2} \mathbf{a}^{\mathrm{NO}}$ in each solvent at $25{ }^{\circ} \mathrm{C}$. The plots for $(S)-\mathbf{2} \mathbf{a}^{\mathrm{NO}}$ are identical to those shown in Figure S6a. The corresponding time-dependent $\mathrm{CD}$ and absorption spectral changes at $25^{\circ} \mathrm{C}$ of $(S)$ $\mathbf{2} \mathbf{a}^{\mathrm{NO}}$ and poly- $(S)-\mathbf{2} \mathbf{a}^{\mathrm{NO}}$ are also shown in Figures S6b-d and S15, respectively. [2 $\mathbf{a}^{\mathrm{NO}}$ or polymer] $=$ $1.0 \mathrm{mM}$. 

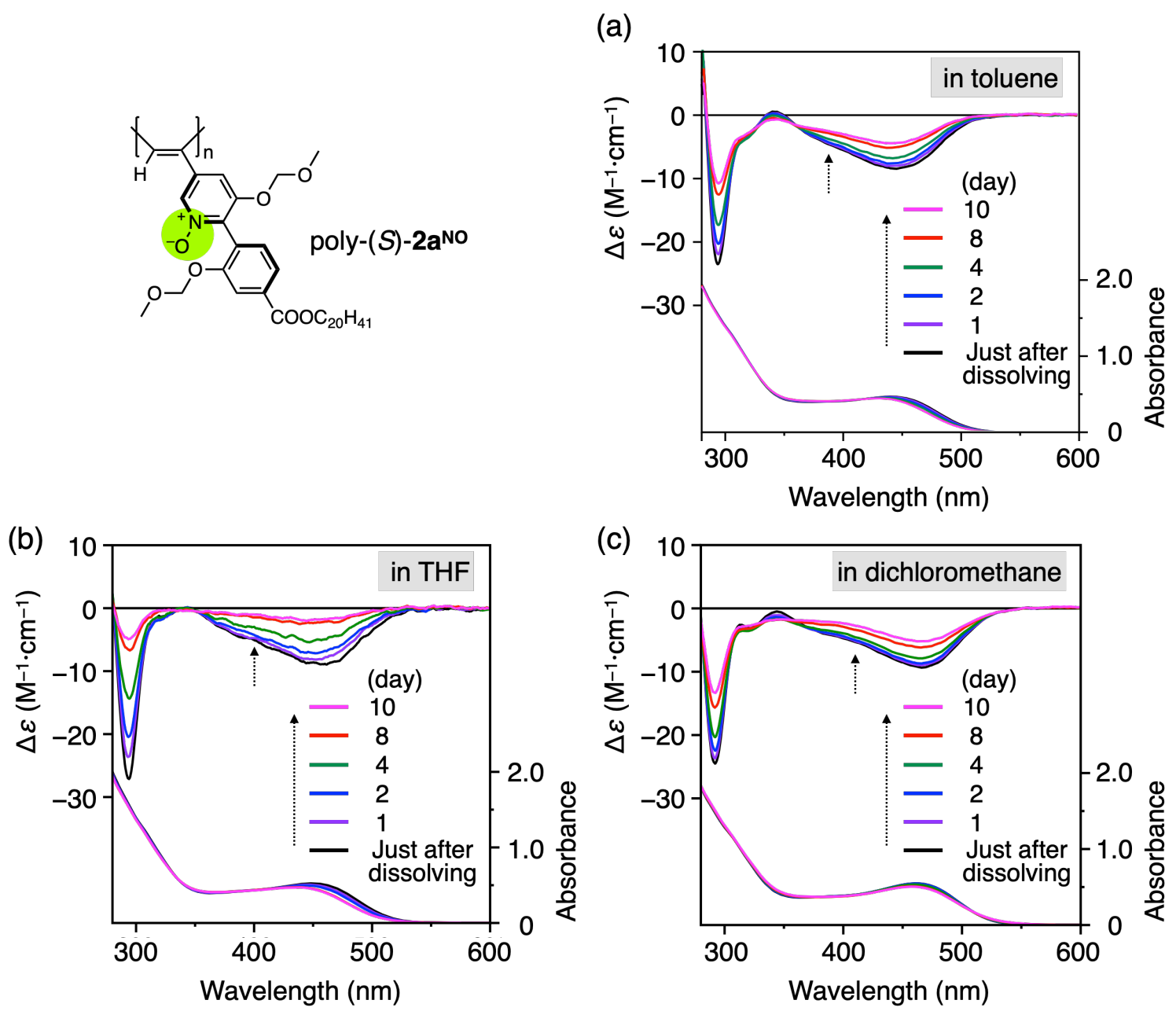

Figure S15. Time-dependent $C D$ and absorption spectral changes of poly- $(S)-\mathbf{2} \mathbf{a}^{\text {NO }}$ in toluene (a), THF (b), and dichloromethane (c) at $25{ }^{\circ} \mathrm{C}$ after allowing to stand at $25{ }^{\circ} \mathrm{C}$ for a specified length of time. $[$ Polymer $]=1.0 \mathrm{mM}$. 


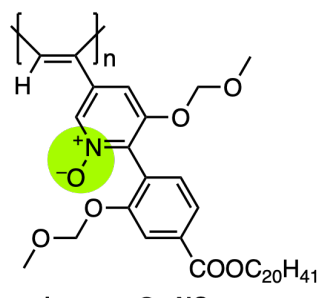

poly-rac-2a $\mathrm{a}^{\mathrm{NO}}$

a
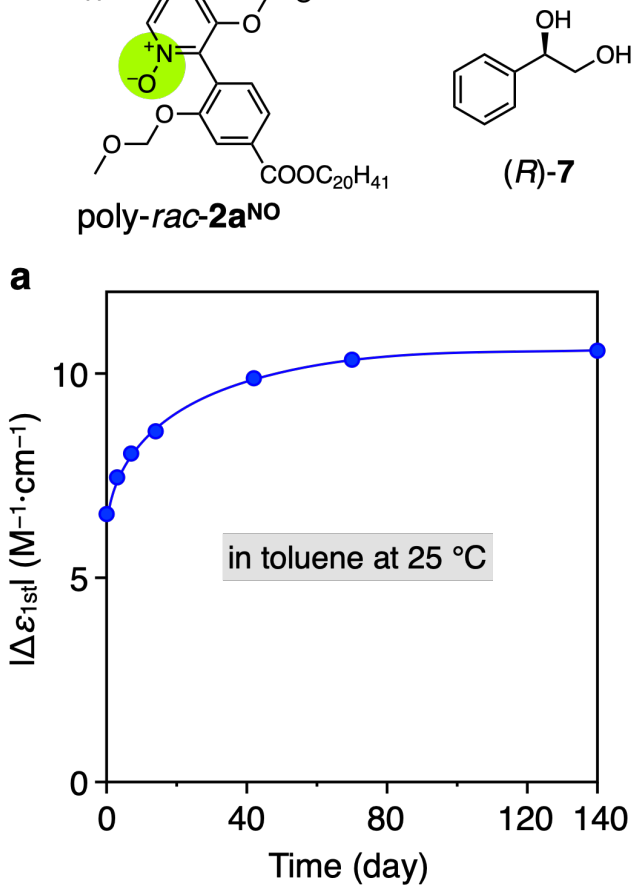

- Just after dissolving in toluene containing $(R)-7$ (20 equiv)

Standing at $25^{\circ} \mathrm{C}$ for $3-140$ days

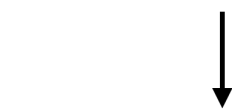

CD measurement at $25^{\circ} \mathrm{C}$

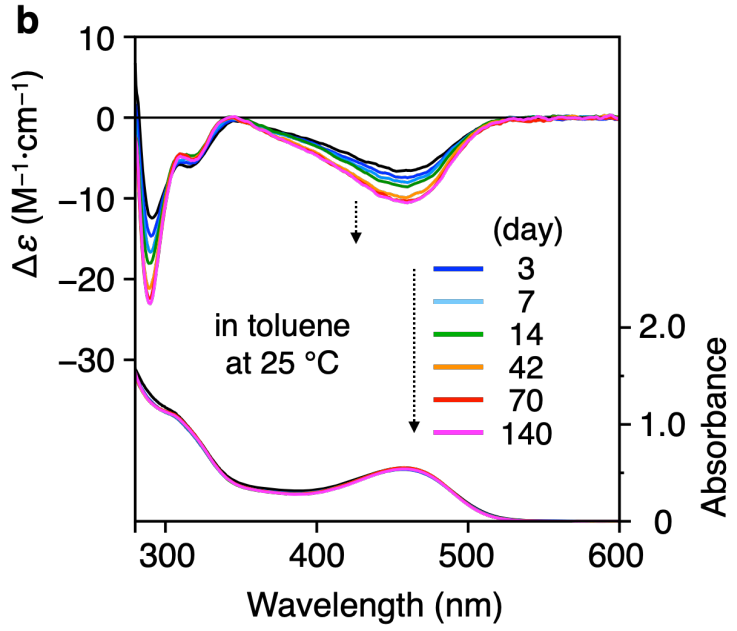

Figure S16. (a) Time-dependent ICD intensity $\left(\left|\Delta \varepsilon_{1 \mathrm{st}}\right|\right)$ changes of poly-rac-2a ${ }^{\mathbf{N O}}$ with $(R)-\mathbf{7}$ in toluene $\left([7] /\left[\right.\right.$ poly-rac-2a $\left.\left.{ }^{\mathrm{NO}}\right]=20\right)$ at $25^{\circ} \mathrm{C}$. The corresponding $\mathrm{CD}$ and absorption spectra are also shown in (b). [Polymer $]=1.0 \mathrm{mM}$. 


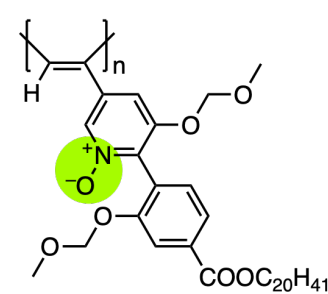

poly-rac-2aNO

(a)

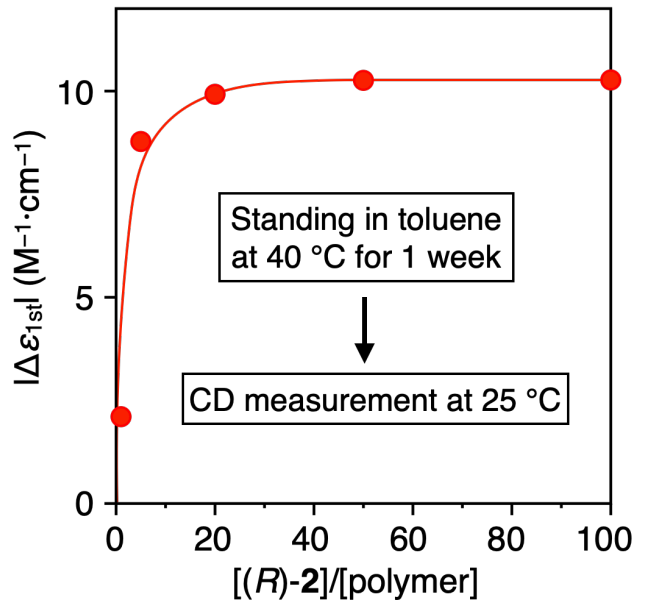

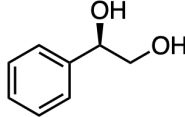

$(R)-7$

(b)

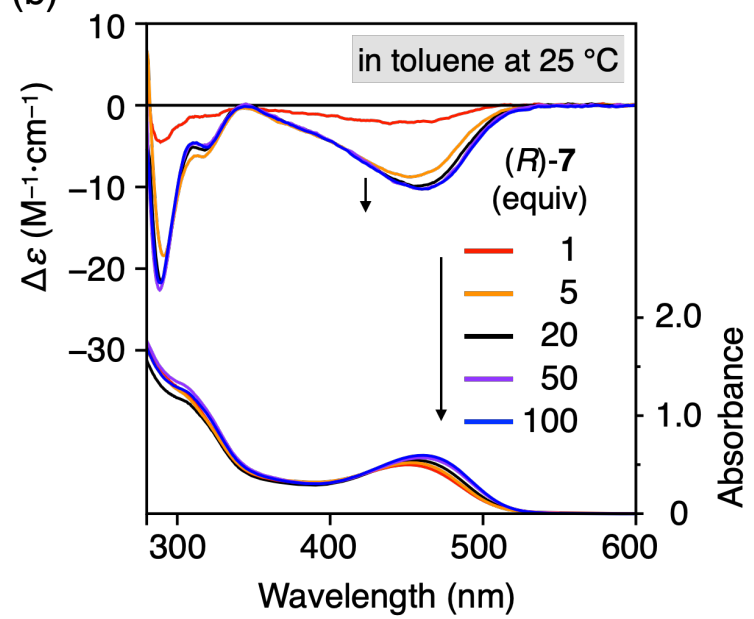

Figure S17. (a) CD titration curve $\left(\left|\Delta \varepsilon_{1 \mathrm{st}}\right|\right)$ of poly-rac-2a ${ }^{\mathrm{NO}}$ with $(R)-7$ in toluene measured at $25{ }^{\circ} \mathrm{C}$ after allowing to stand at $40^{\circ} \mathrm{C}$ for seven days. The corresponding $\mathrm{CD}$ and absorption spectra are also shown in (b). [Polymer] $=1.0 \mathrm{mM}$. 
(a) poly-rac-2a ${ }^{\mathrm{NO}}$ with $(R)-7$ (0.1 equiv)

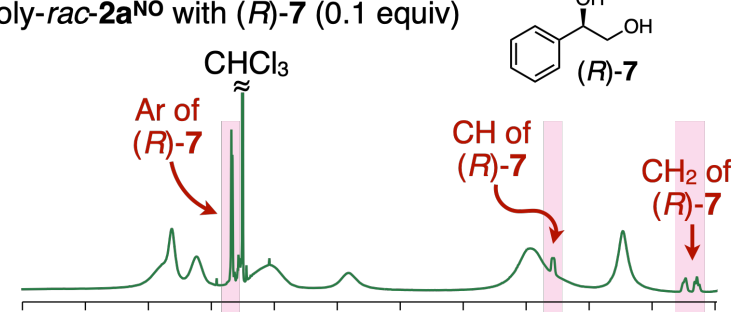

(c) $h_{((A))}$-poly-derac-2a NO isolated from toluene/( $\left.R\right)^{-7}$

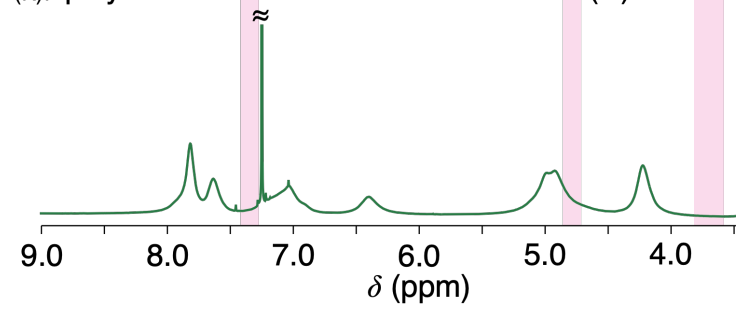

(b) poly-rac-2a No with ( $S$ )-7 (0.1 equiv)

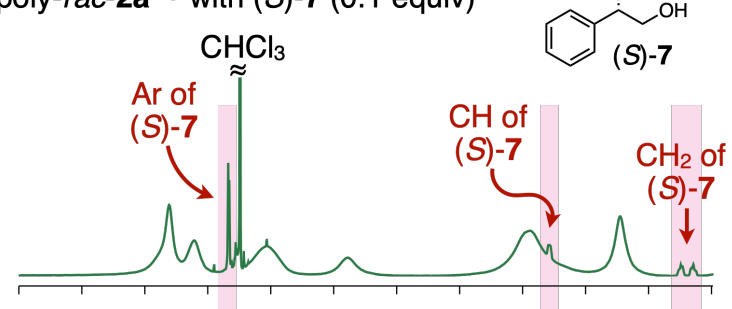

(d) $h_{(S)}$-poly-derac-2anO isolated from toluene/(S)-7

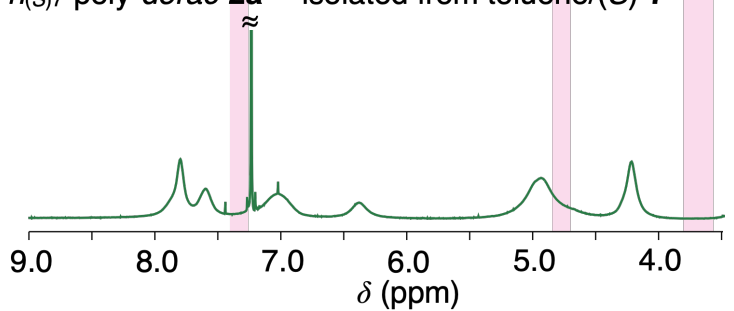

Figure S18. ${ }^{1} \mathrm{H}$ NMR spectra of poly-rac-2a ${ }^{\text {NO }}$ in the presence of $(R)-7$ (a) and $(S)-7$ (b) ([7]/[poly-

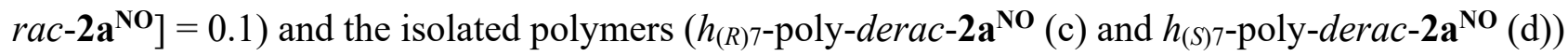
with helicity memory, measured in $\mathrm{CDCl}_{3}$ at $50^{\circ} \mathrm{C}$. 
(i): poly-rac-2aNO $(1.0 \mathrm{mM})$ with $(R)-7(50 \mathrm{mM})(\longrightarrow)$

(ii): Isolated $h_{(R) 7}$-poly-derac-2aNO (ca. $1 \mathrm{mM}$ ) from i (-- - -)

(i): poly-(S)-2ano (1.0 mM) (-)

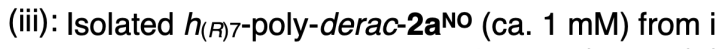

(a)

with rac-7 $(50 \mathrm{mM})(-\ldots)$

(ii): poly-(S)-2aNo (1.0 mM) with rac-7 $(50 \mathrm{mM})(----)$

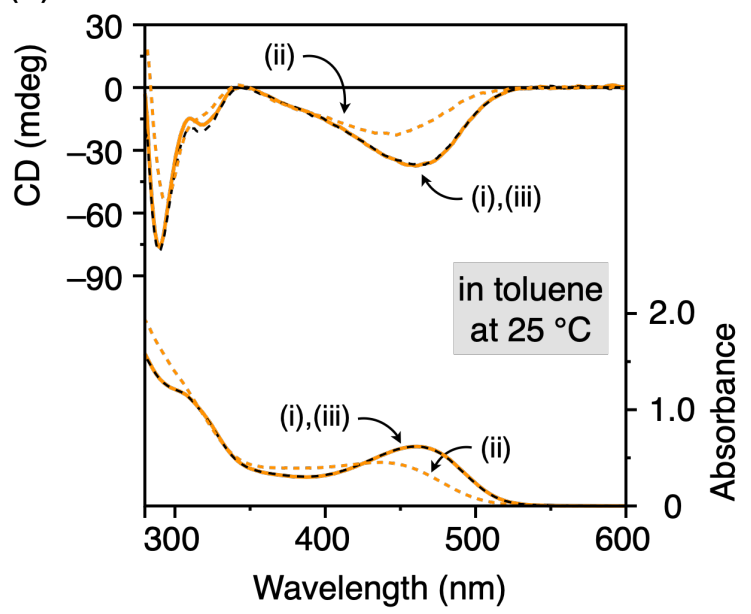

(b)

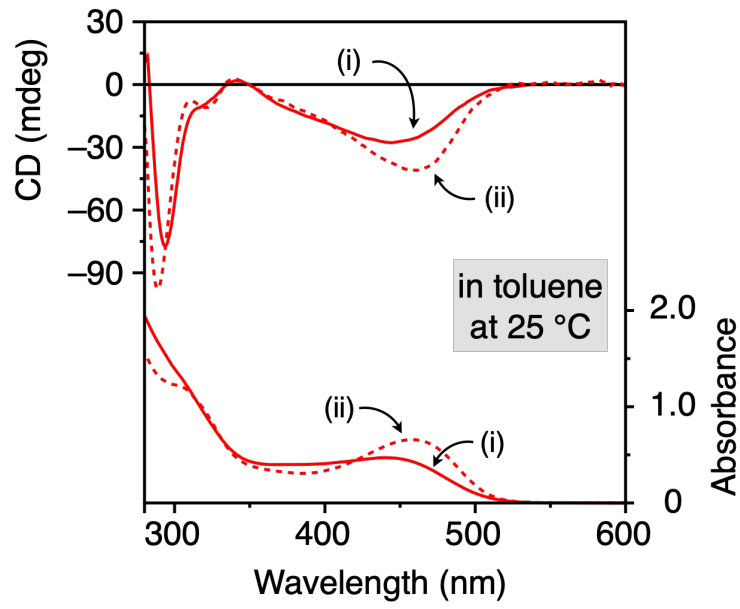

(i): poly-rac-2ano $(1.0 \mathrm{mM})(-)$

(c)

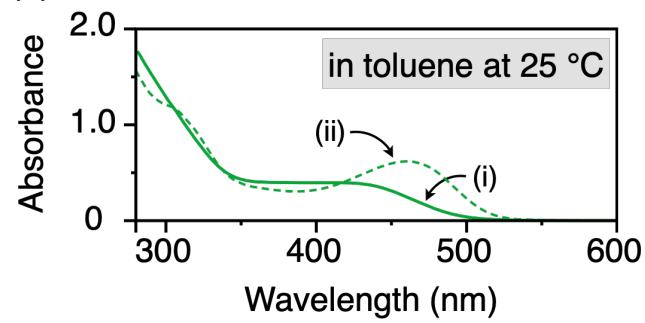

Figure S19. (a) CD and absorption spectra of poly-rac-2a $\mathbf{a}^{\mathrm{NO}}$ in the presence of $(R)-7$ in toluene ([(R)$7] /\left[\right.$ poly-rac-2a $\left.\mathbf{a}^{\mathrm{NO}}\right]=50$ ) measured at $25{ }^{\circ} \mathrm{C}$ after allowing to stand at $40{ }^{\circ} \mathrm{C}$ for seven days, and the isolated $h_{(R) 7-p o l y-d e r a c-2 a^{N O}}$ recovered from i (ii), just after dissolving in toluene in the absence (ii) and presence (iii) of $\mathrm{rac}-7$ at $25^{\circ} \mathrm{C}([\mathrm{rac}-7] /[$ polymer $]=50)$. (b,c) $\mathrm{CD}$ and absorption spectra of poly$(S)-\mathbf{2} \mathbf{a}^{\mathrm{NO}}(\mathrm{b})$ and absorption spectra of poly-rac-2 $\mathbf{a}^{\mathrm{NO}}$ (c) just after dissolving in toluene in the absence (i) and presence (ii) of $\mathrm{rac}-7$ at $25^{\circ} \mathrm{C}([\mathrm{rac}-7] /[$ polymer $]=50)$. [Polymer $]=\mathrm{ca} .1 \mathrm{mM}$. 


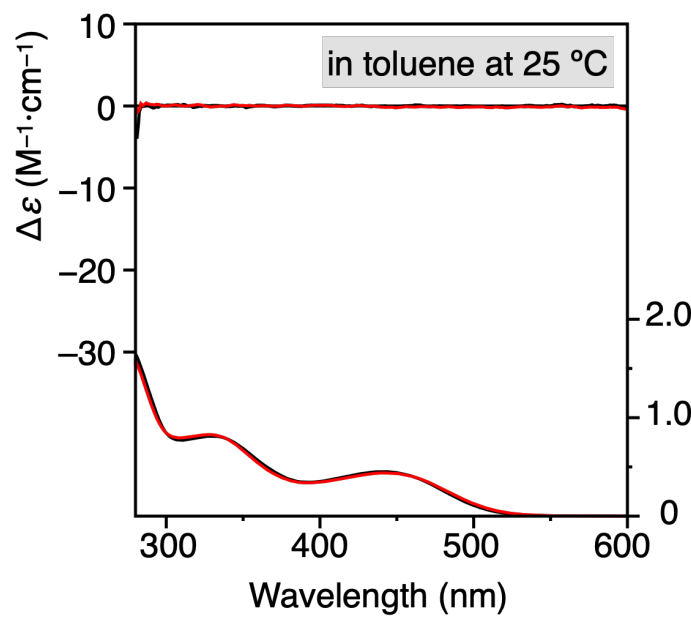

(i): Just after dissolving poly-rac-2b NO $^{2}$

in toluene containing

$(R)-7$ (50 equiv) $(-)$

Standing at $40^{\circ} \mathrm{C}$

(ii): CD measurement at $25^{\circ} \mathrm{C}(-)$
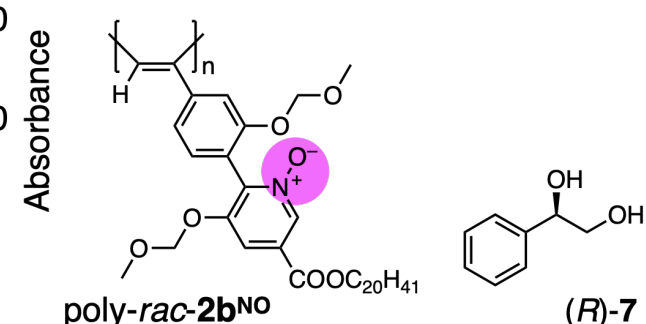

$(R)-7$

Figure S20. CD and absorption spectra of poly-rac-2 $\mathbf{b}^{\text {NO }}$ in the presence of $(R)-7$ in toluene $([(R)-$ 7] $/\left[\right.$ poly-rac-2 $\left.\mathbf{b}^{\mathrm{NO}}\right]=50$ ) measured at $25{ }^{\circ} \mathrm{C}$ before (i) and after (ii) allowing to stand at $40{ }^{\circ} \mathrm{C}$ for seven days. $[$ Polymer $]=1.0 \mathrm{mM}$.
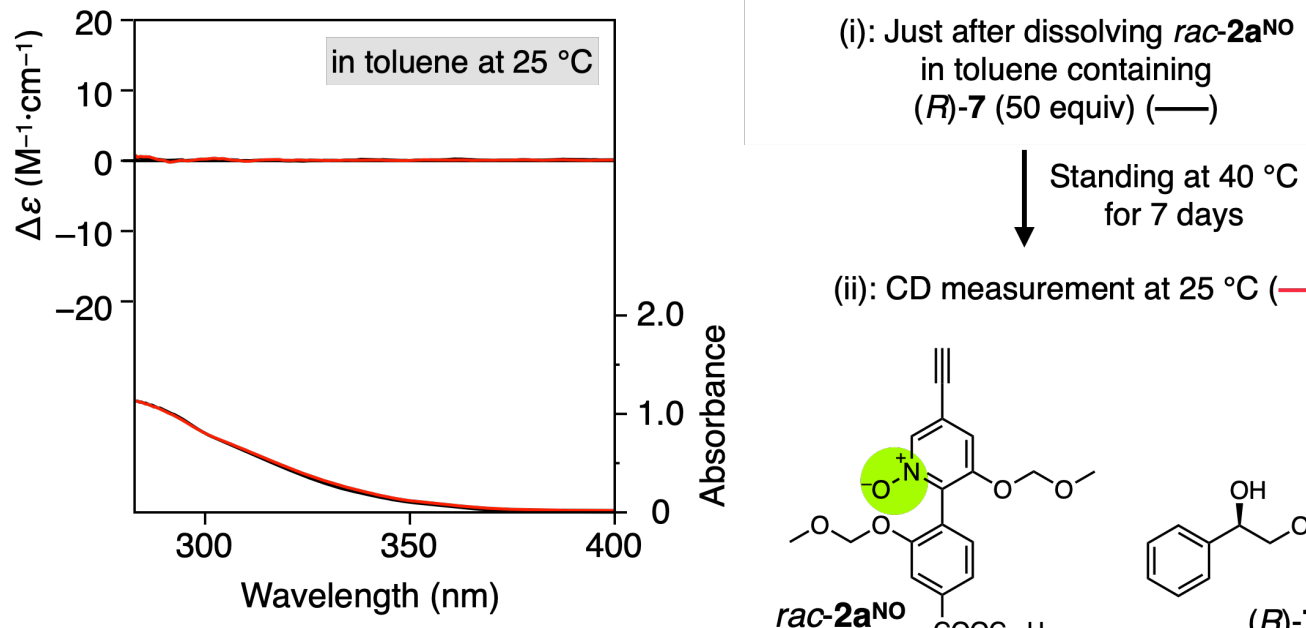

(ii): CD measurement at $25^{\circ} \mathrm{C}(-)$

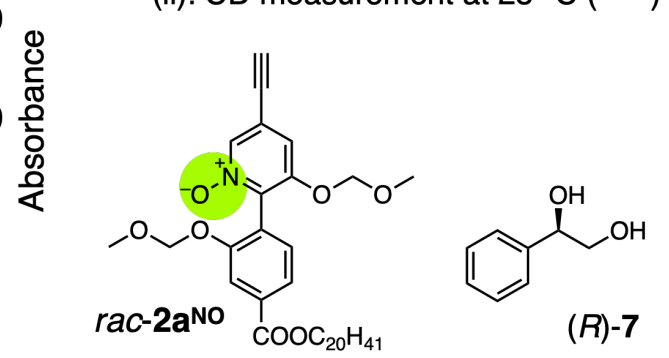

Figure S21. CD and absorption spectra of rac-2a ${ }^{\text {NO }}$ in the presence of $(R)-7$ in toluene $([(R)-7] /[\mathrm{rac}$ $\left.\mathbf{2 a}^{\mathrm{NO}}\right]=50$ ) measured at $25^{\circ} \mathrm{C}$ before (i) and after (ii) allowing to stand at $40{ }^{\circ} \mathrm{C}$ for seven days. [ $\mathrm{rac}$ $\left.\mathbf{2 a}^{\mathrm{NO}}\right]=1.0 \mathrm{mM}$. 


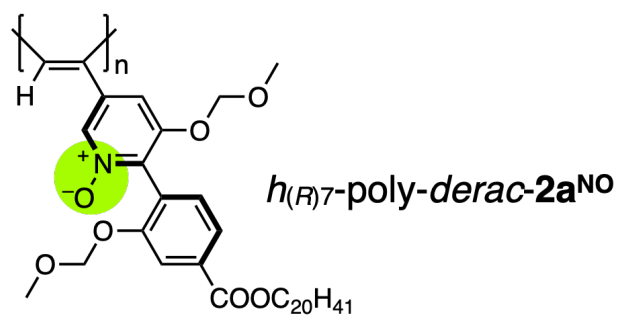

(a)

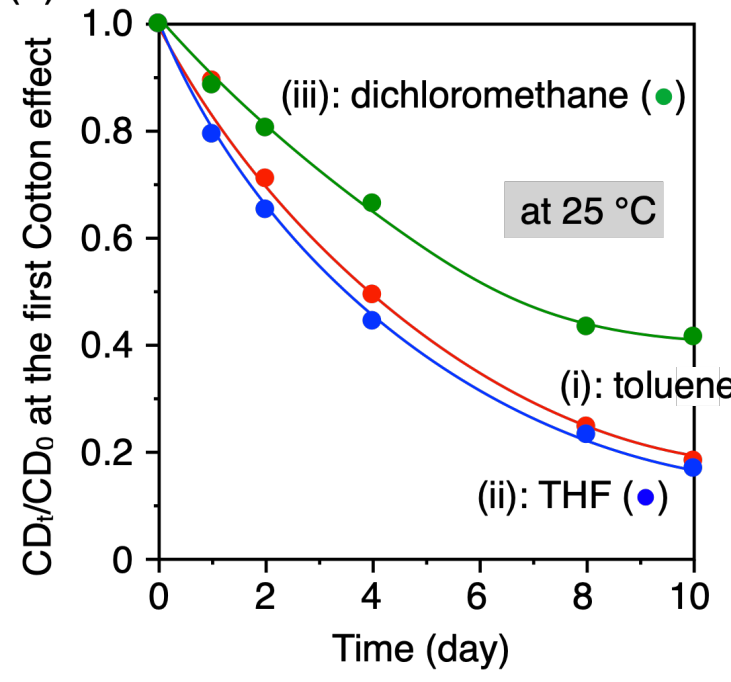

(c)

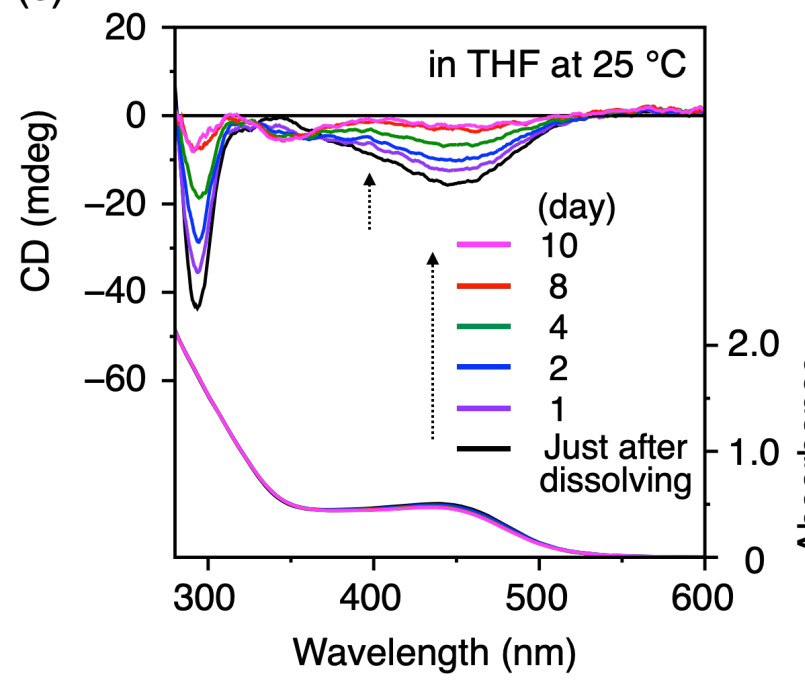

(b)

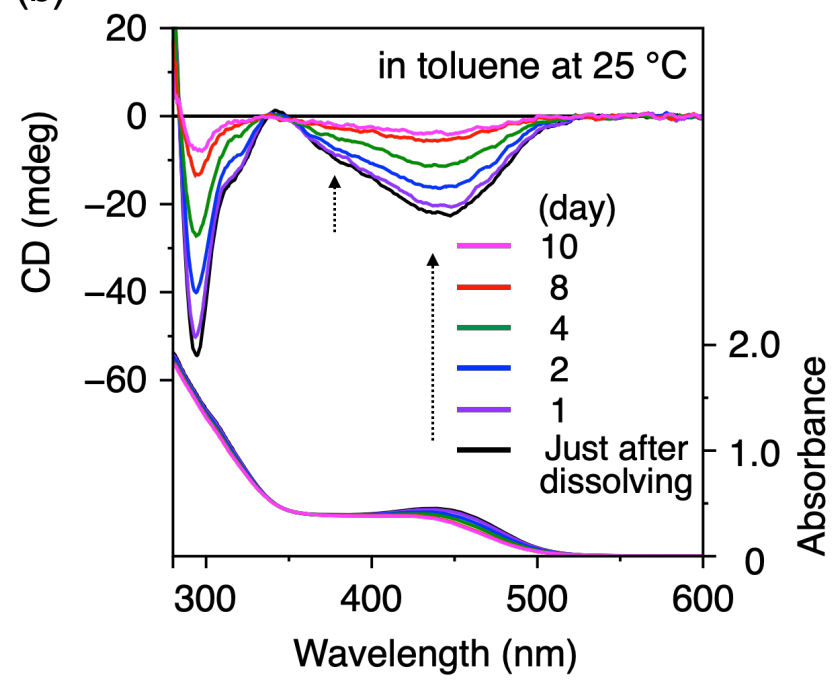

(d)

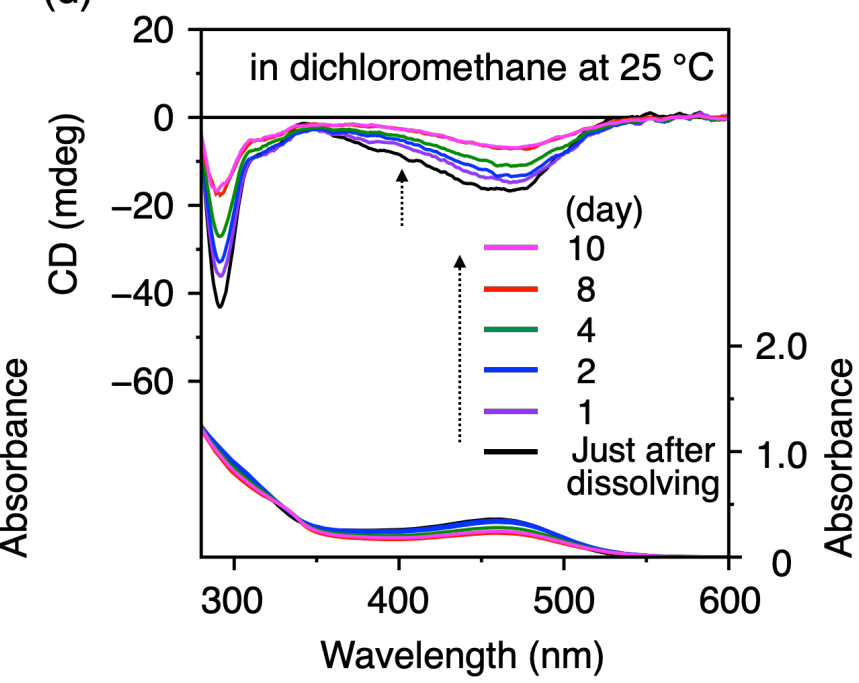

Figure S22. (a) Plots of the time-dependent $C D$ intensity changes at the first Cotton effect $\left(\mathrm{CD}_{t} / \mathrm{CD}_{0}\right)$ of the helicity-memorized $h_{(R) 7}$-poly-derac-2a $\mathbf{a}^{\text {NO }}$ measured in toluene (i), THF (ii), and dichloromethane (iii) at $25{ }^{\circ} \mathrm{C} . \mathrm{CD}_{0}$ represents the initial CD intensity of $h_{(R) 7-\text { poly-derac-2a }} \mathbf{a}^{\mathrm{NO}}$ in each solvent at $25{ }^{\circ} \mathrm{C}$. The corresponding time-dependent $\mathrm{CD}$ and absorption spectral changes at $25^{\circ} \mathrm{C}$ are also shown in (b-d). [Polymer] $=1.0 \mathrm{mM}$. 
<smiles>C=CCC(O)c1ccccc1</smiles>
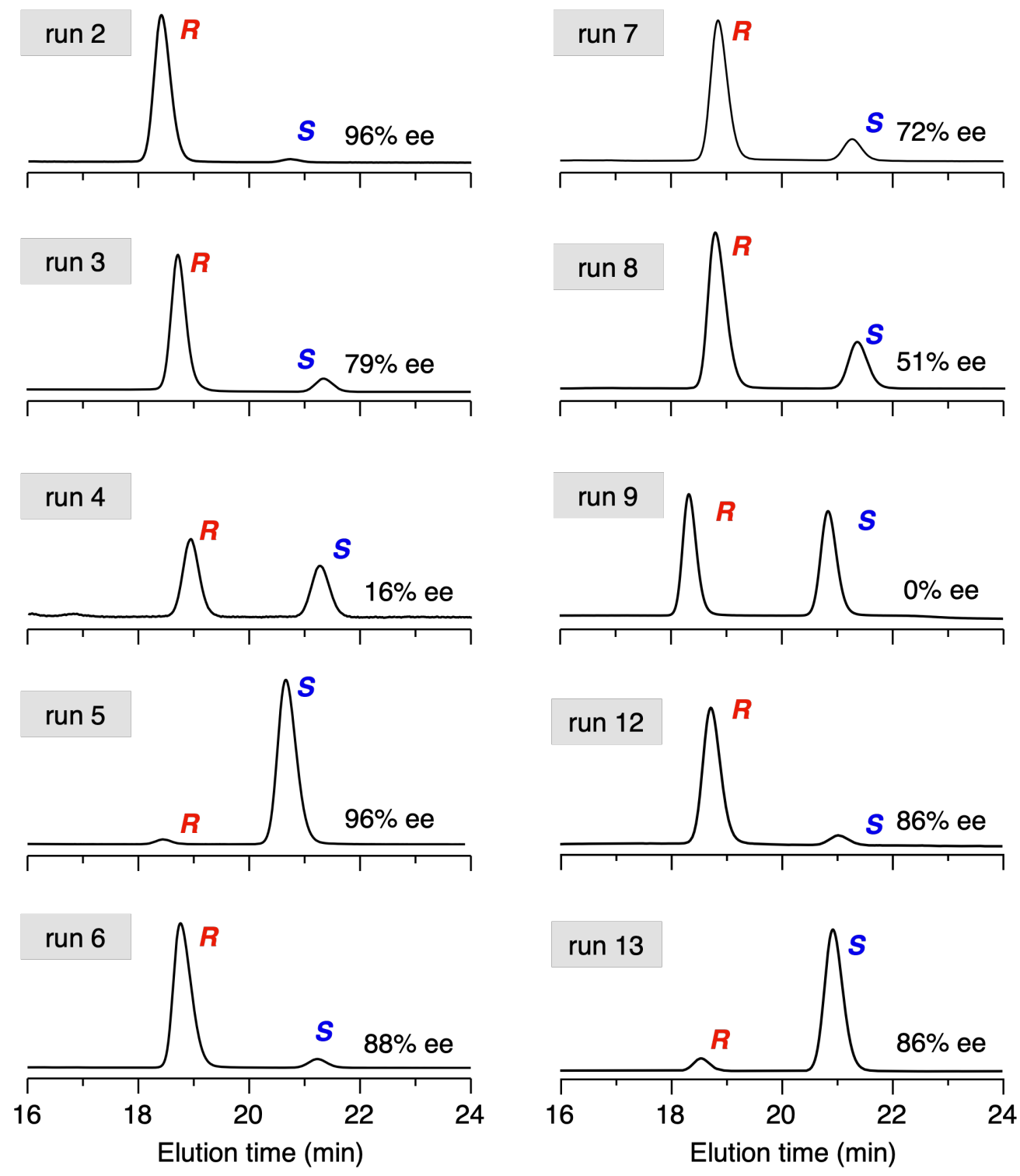

Figure S23. Chromatograms for the resolution of $\mathbf{9}$ produced after enantioselective allylation reactions (Table 3). Chromatographic conditions: column, CHIRALCEL OD-H $(0.46 \mathrm{~cm}$ (i.d.) $\times 25$ $\mathrm{cm})$; eluent, $n$-hexane/2-propanol $(98 / 2, \mathrm{v} / \mathrm{v})$; flow rate, $0.8 \mathrm{~mL} / \mathrm{min}$; temperature, room temperature. 


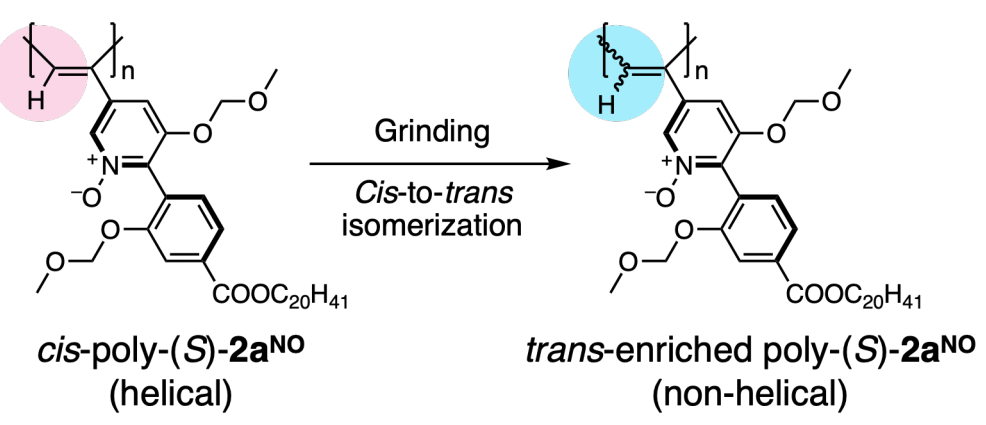

(a) cis-poly-(S)-2a No

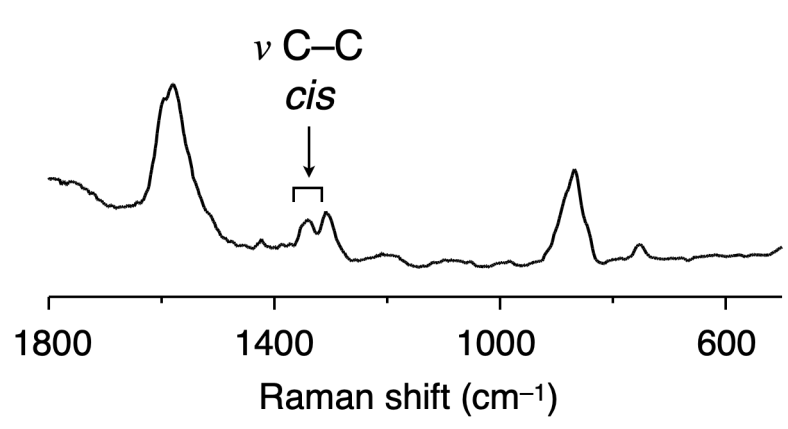

(b) trans-enriched poly-(S)-2ano

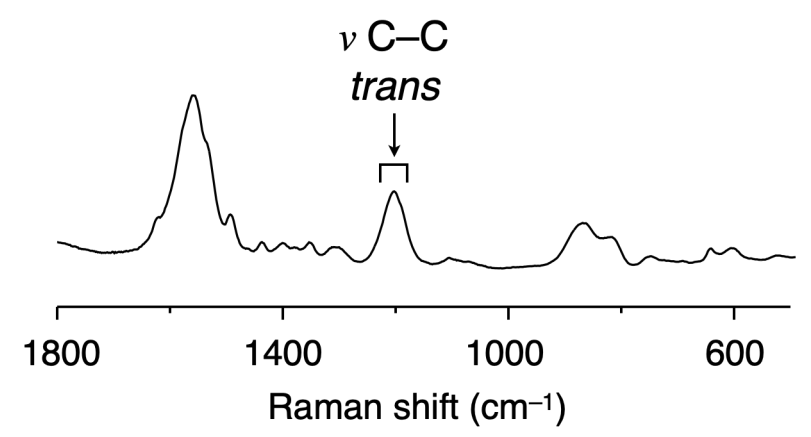

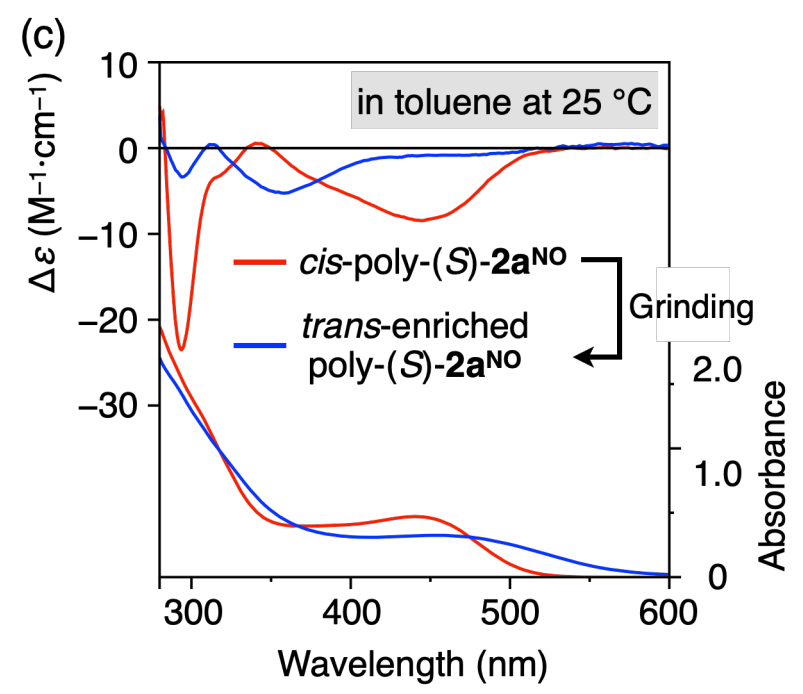

Figure S24. (a,b) Laser Raman spectra of cis-poly-(S)-2a ${ }^{\mathbf{N O}}$ (a) and trans-enriched poly-(S)-2a ${ }^{\mathbf{N O}}(\mathrm{b})$ in the solid state at room temperature. (c) $\mathrm{CD}$ and absorption spectra of poly-(S)-2 $\mathbf{a}^{\mathrm{NO}}$ before (i; cis-

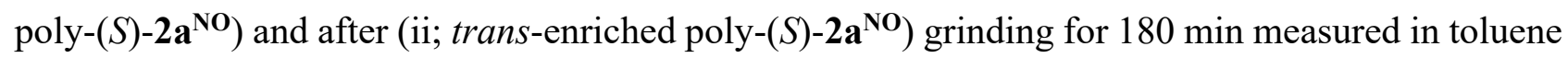
at $25^{\circ} \mathrm{C}$. [Polymer] $=1.0 \mathrm{mM}$. 

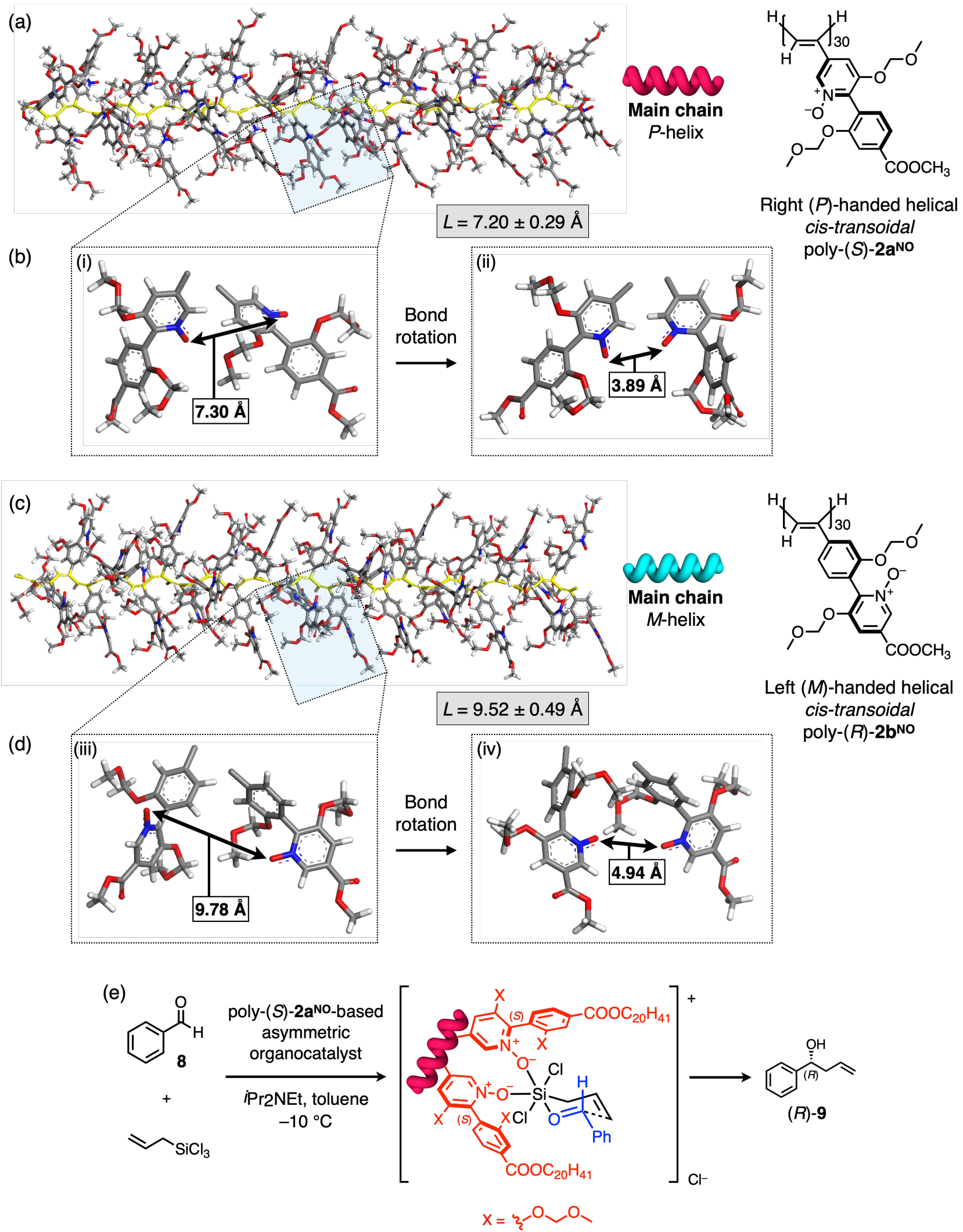

Figure S25. (a,c) Possible right- and left-handed helical structures (30-mer) of cis-transoidal poly$(S)-\mathbf{2} \mathbf{a}^{\mathrm{NO}}$ (a) and poly- $(R)-\mathbf{2} \mathbf{b}^{\mathrm{NO}}(\mathrm{c})$, respectively, optimized by molecular mechanics calculations. For simplicity, the icosyloxycarbonyl $\left(\mathrm{C}_{20} \mathrm{H}_{41} \mathrm{OCO}\right)$ groups of the pendants were replaced by the methoxycarbonyl groups. The structures are represented by capped-stick models and the main-chain atoms are shown in a different color (yellow) for clarity. Average interatomic distances $(L)$ between 
the oxygen atoms of the nearest-neighbor $N$-oxide moieties at the central region (10-mer) are also shown. (b,d) The relative arrangements of the nearest-neighbor pendant units of poly- $(S)-\mathbf{2} \mathbf{a}^{\mathbf{N O}}$ and poly- $(R)-\mathbf{2} \mathbf{b}^{\text {NO }}$ taken from (a) and (c), respectively, before (i and iii) and after (ii and iv) rotation around a single bond between the polyacetylene backbone and the nearest-neighbor pendant units, so that the two oxygen atoms of the $N$-oxide moieties are proximate to each other. (e) A possible cyclic cationic transition state model for the asymmetric allylation of benzaldehyde (8) with allyltrichlorosilane catalyzed by the one-handed helical cis-transoidal poly-(S)-2a $\mathbf{a}^{\text {NO }}$ to afford $(R)-\mathbf{9}$.

We note that such a cationic transition state model through bidentate chelation (Figure S25e) has been proposed to be operative in polar solvents, such as dichloromethane when chiral bipyridine $N, N^{\prime}$-dioxides were used as catalysts. ${ }^{\mathrm{S} 22-26}$ In less polar solvents, such as toluene, a neutral transition state model via coordination of one of the $N$-oxide moieties of chiral bipyridine $N, N$ '-dioxides has also been proposed for another possible reaction mechanism, producing the optically active alcohol, whose chirality was opposite to that produced in dichloromethane as predicted by theoretical calculations. $^{\text {S27-29 }}$ In contrast, poly-(S)-2a ${ }^{\text {NO }}$ enantioselectively catalyzed the allylation of benzaldehyde independent of the solvents, giving the same $(R)$-alcohol in dichloromethane as well as in toluene (runs 2 and 3, Table 3). Therefore, the cyclic cationic transition state model through a cooperative chelation by the nearest-neighbor pendant $N$-oxide moieties was used for explaining the emergence of highly enantioselective catalytic activity observed for poly- $(S)-\mathbf{2 a} \mathbf{a}^{\text {NO }}$. 
(a) poly-rac-2a $\mathrm{a}^{\mathrm{NO}}$

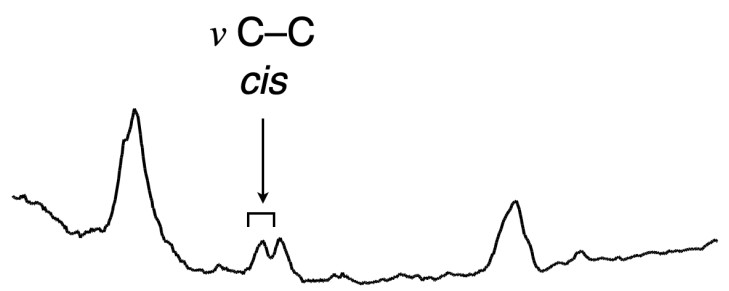

(b) poly- $(R)-2 a^{\text {NO }}$

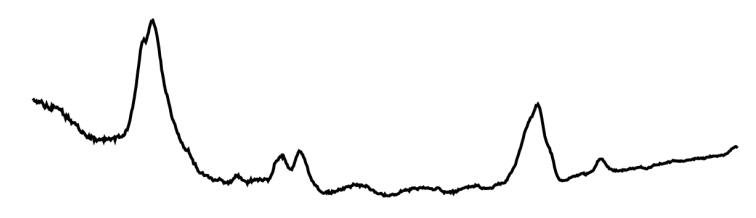

(e) $\operatorname{poly}\left((S)-2 \mathrm{a}^{\mathrm{NO}_{0.815}-C O-}(R)-2 \mathrm{a}^{\mathrm{NO}}{ }_{0.185}\right)$

(b) poly-(R)-2ano

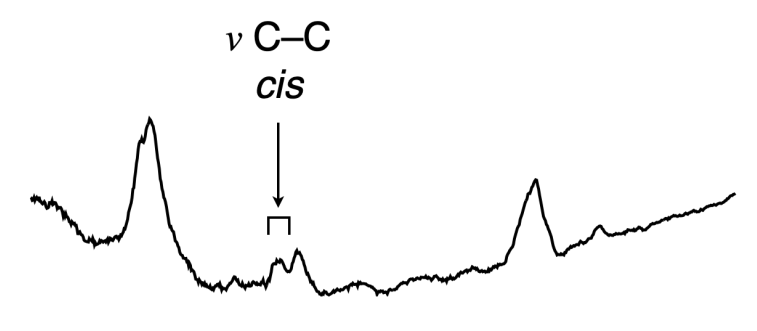

(c) poly-rac-2bNo

(f) $\operatorname{poly}\left((S)-2 \mathrm{a}^{\mathrm{NO}} 0.71-\mathrm{CO}-(R)-2 \mathrm{a}^{\mathrm{NO}}{ }_{0.29}\right)$
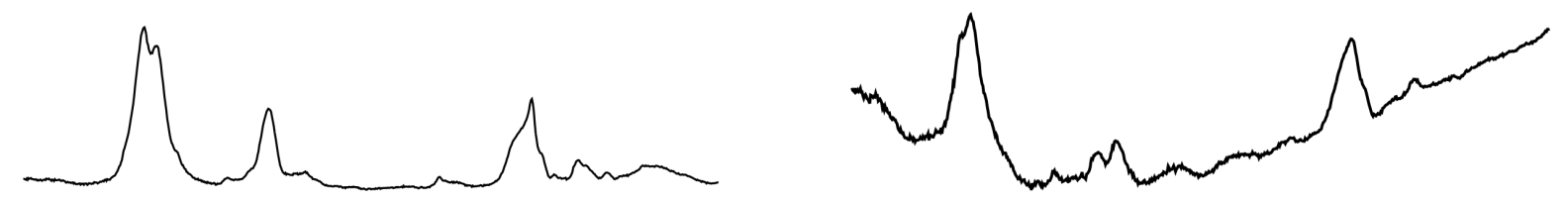

(d) poly- $(R)-2 b^{N O}$

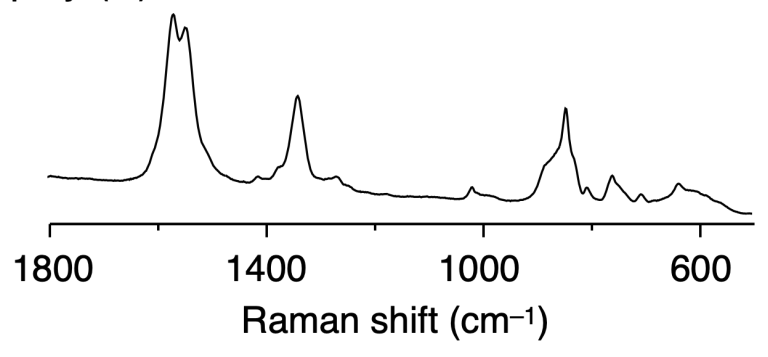

(g) $\operatorname{poly}\left((S)-2 \mathrm{a}^{\mathrm{NO}_{0.61}-\mathrm{CO}-(R)-2 \mathrm{a}^{\mathrm{NO}}}{ }_{0.39}\right)$

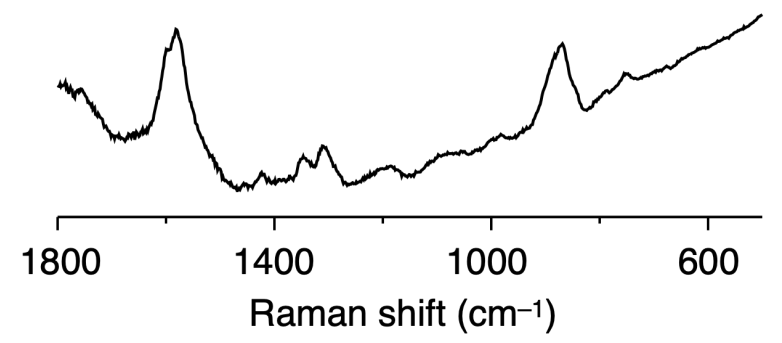

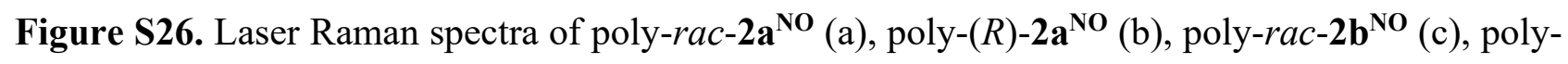
$(R)-\mathbf{2} \mathbf{b}^{\mathbf{N O}}(\mathrm{d}), \operatorname{poly}\left((S)-\mathbf{2} \mathbf{a}^{\mathbf{N O}}{ }_{0.815}-c o-(R)-\mathbf{2} \mathbf{a}^{\mathbf{N O}} 0.185\right)$ (e), $\operatorname{poly}\left((S)-\mathbf{2} \mathbf{a}^{\mathbf{N O}} 0.71-c o-(R)-\mathbf{2} \mathbf{a}^{\mathbf{N O}} 0.29\right)$ (f), and $\operatorname{poly}\left((S)-\mathbf{2} \mathbf{a}^{\mathrm{NO}_{0.61}-c o-(R)-\mathbf{2} \mathbf{a}^{\mathrm{NO}}}{ }_{0.39}\right)(\mathrm{g})$ in the solid state at room temperature. For the spectrum of poly$(S)-\mathbf{2} \mathbf{a}^{\mathbf{N O}}$, see Figure S24a. 

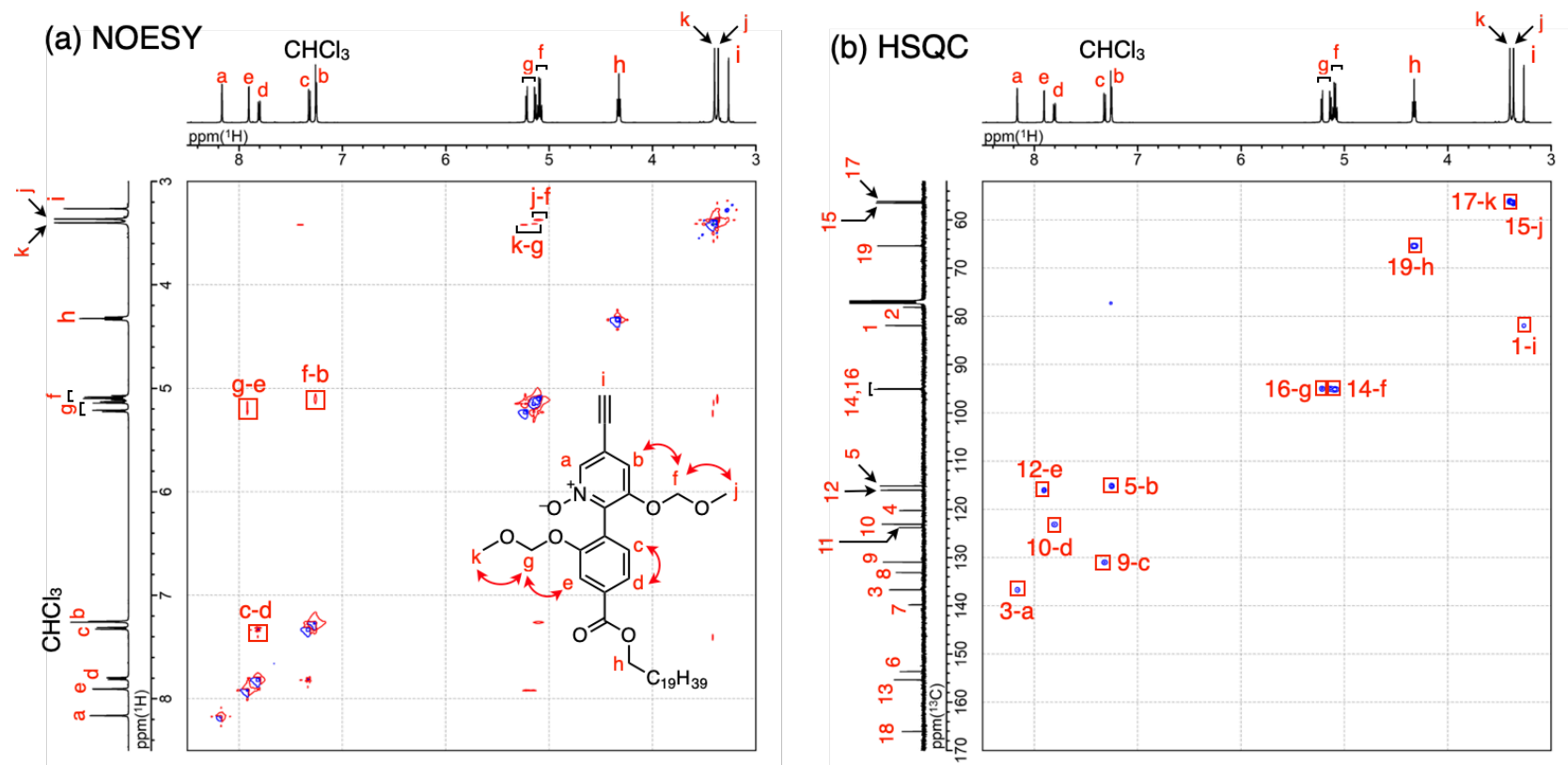

(c) $\mathrm{HMBC} \quad \mathrm{CHCl}_{3}$
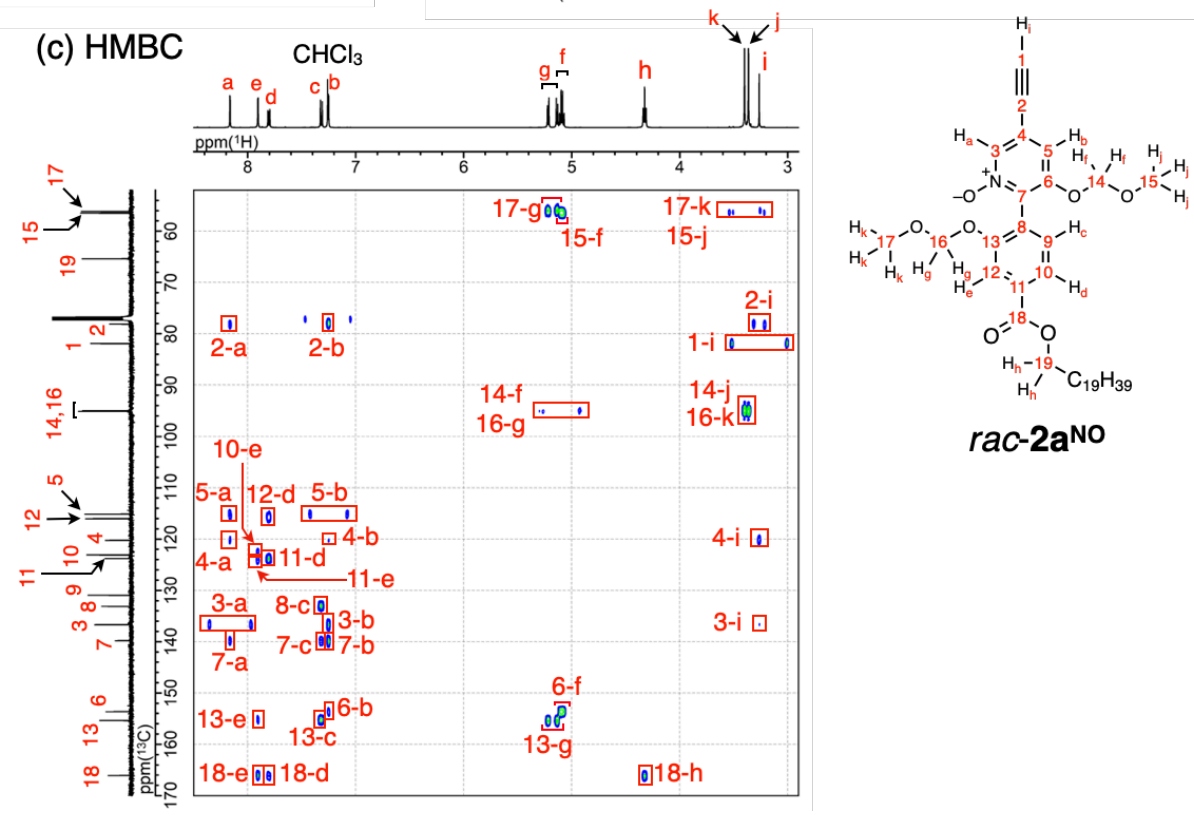

rac-2aNo

Figure S27. Partial NOESY (a), HSQC (b), and HMBC (c) spectra $\left(500 \mathrm{MHz}, \mathrm{CDCl}_{3}, 25^{\circ} \mathrm{C}\right.$, mixing time $=500 \mathrm{~ms}(\mathrm{NOESY}))$ of $r a c-\mathbf{2} \mathbf{a}^{\text {NO }}$. 

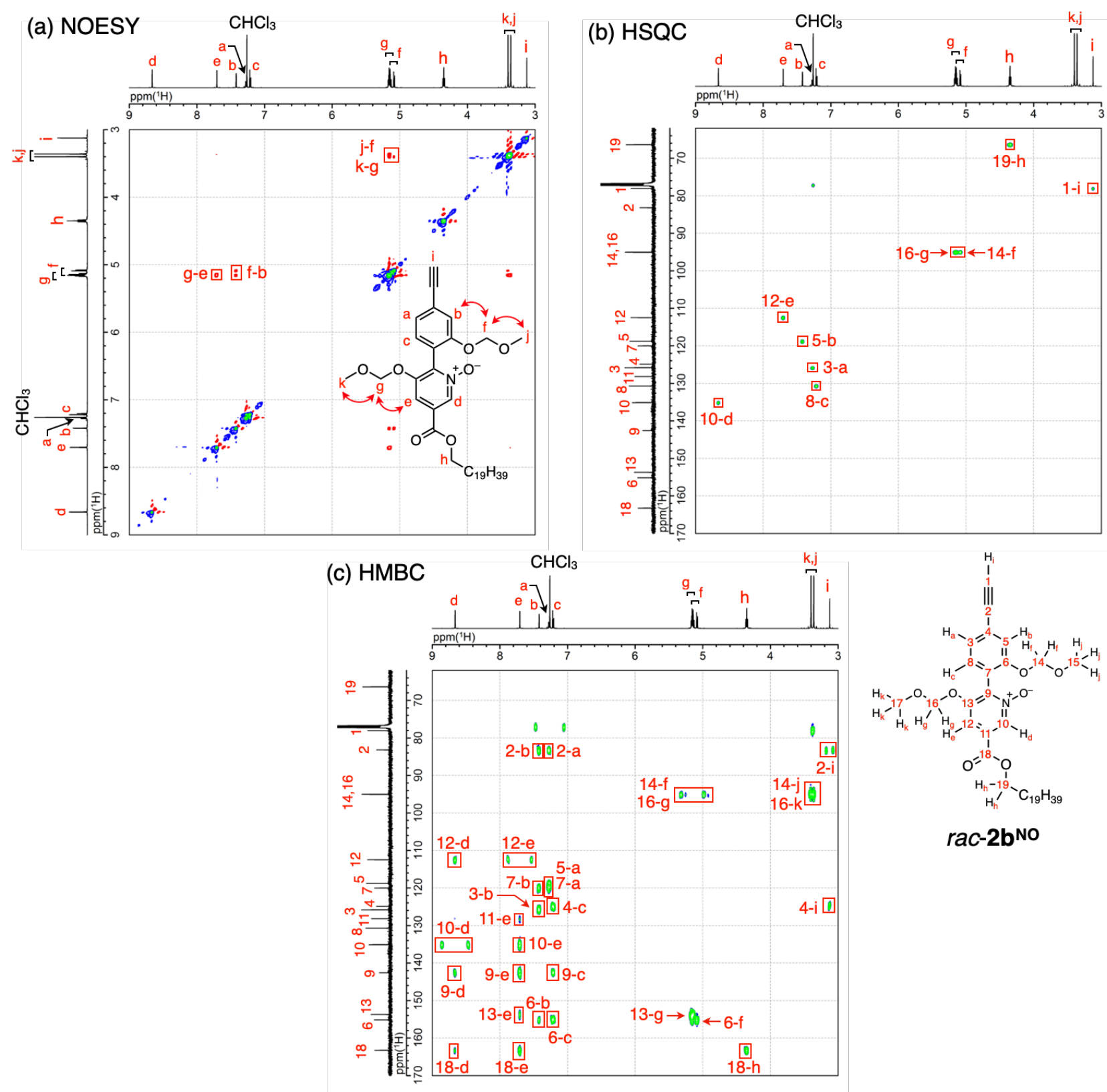

Figure S28. Partial NOESY (a), HSQC (b), and HMBC (c) spectra $\left(500 \mathrm{MHz}, \mathrm{CDCl}_{3}, 25^{\circ} \mathrm{C}\right.$, mixing time $=500 \mathrm{~ms}(\mathrm{NOESY}))$ of $r a c-\mathbf{2} \mathbf{b}^{\text {NO }}$. 
(a) CosY
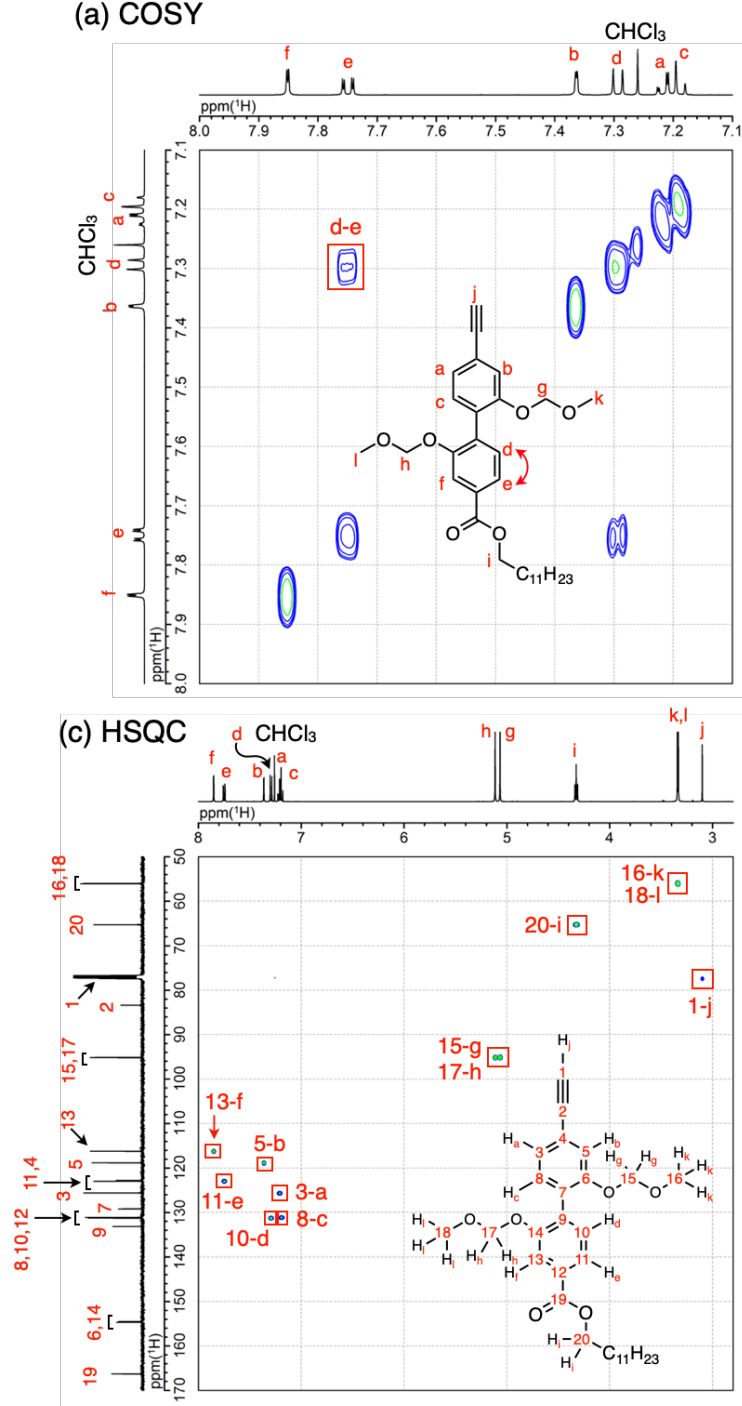

(b) NOESY
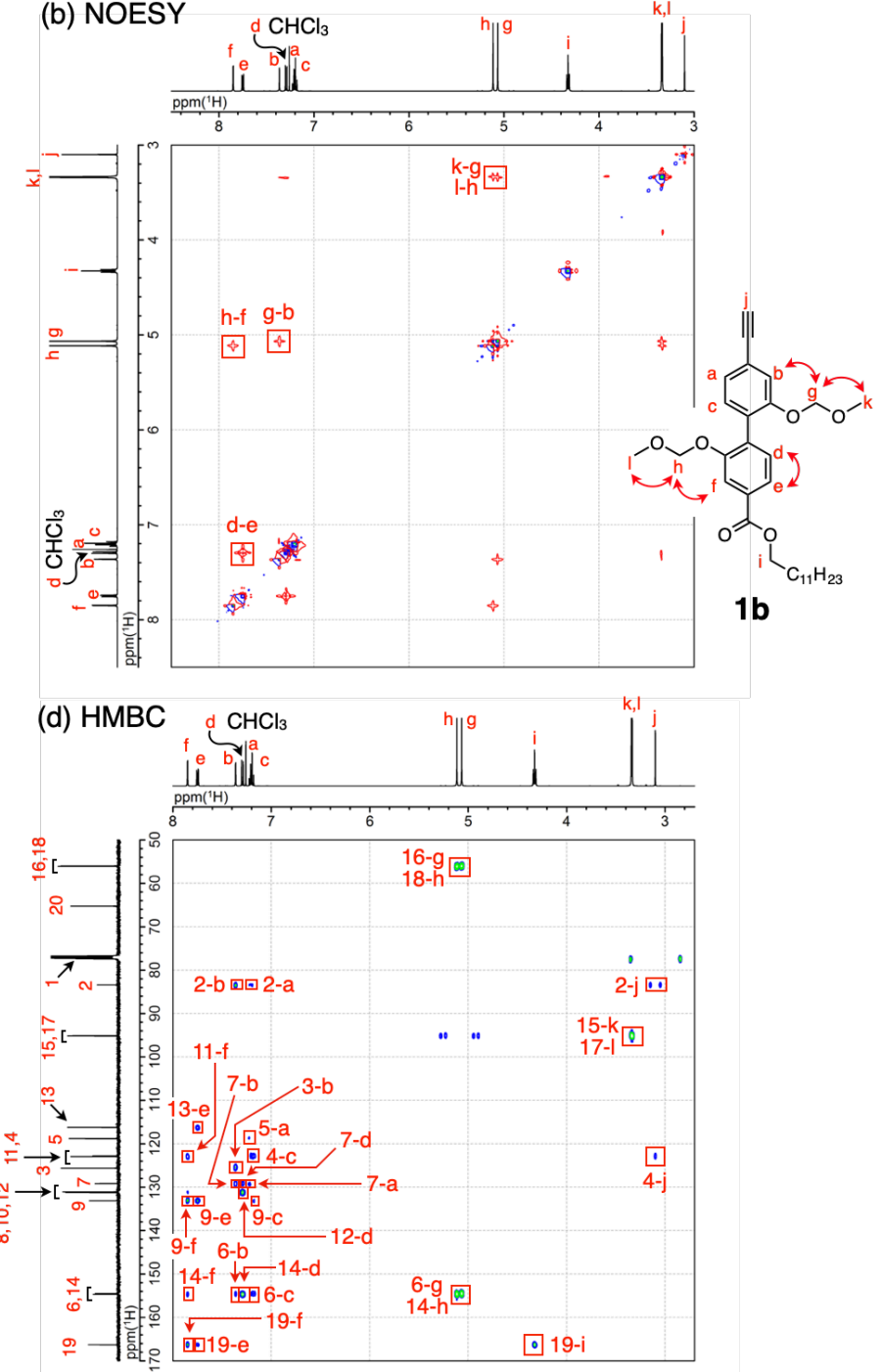

Figure S29. Partial COSY (a), NOESY (b), HSQC (c), and HMBC (d) spectra (500 MHz, $\mathrm{CDCl}_{3}$, $25^{\circ} \mathrm{C}$, mixing time $\left.=500 \mathrm{~ms}(\mathrm{NOESY})\right)$ of $\mathbf{1 b}$. 


\section{Supporting References}

(S1) Ishidate, R.; Shimomura, K.; Ikai, T.; Kanoh, S.; Maeda, K. Macromolecular Helicity Induction and Memory in a Poly(biphenylylacetylene) Bearing an Ester Group and Its Application to a Chiral Stationary Phase for High-performance Liquid Chromatography. Chem. Lett. 2015, 44, 946-948.

(S2) Taniguchi, T.; Yoshida, T.; Echizen, K.; Takayama, K.; Nishimura, T.; Maeda, K. Facile and Versatile Synthesis of End-Functionalized Poly(phenylacetylene)s: A Multicomponent Catalytic System for Well-Controlled Living Polymerization of Phenylacetylenes. Angew. Chem., Int. Ed. 2020, $59,8670-8680$.

(S3) Simionescu, C. I.; Percec, V.; Dumitrescu, S. Polymerization of Acetylenic Derivatives. XXX. Isomers of Polyphenylacetylene. J. Polym. Sci., Polym. Chem. Ed. 1977, 15, 2497-2509.

(S4) Furlani, A.; Napoletano, C.; Russo, M. V.; Feast, W. J. Stereoregular Polyphenylacetylene. Polym. Bull. 1986, 16, 311-317.

(S5) Kishimoto, Y.; Eckerle, P.; Miyatake, T.; Kainosho, M.; Ono, A.; Ikariya, T.; Noyori, R. WellControlled Polymerization of Phenylacetylenes with Organorhodium(i) Complexes: Mechanism and Structure of the Polyenes. J. Am. Chem. Soc. 1999, 121, 12035-12044.

(S6) Percec, V.; Rudick, J. G.; Peterca, M.; Wagner, M.; Obata, M.; Mitchell, C. M.; Cho, W.-D.; Balagurusamy, V. S. K.; Heiney, P. A. Thermoreversible Cis - Cisoidal to Cis - Transoidal Isomerization of Helical Dendronized Polyphenylacetylenes. J. Am. Chem. Soc. 2005, 127, 1525715264.

(S7) Nagai, K.; Sakajiri, K.; Maeda, K.; Okoshi, K.; Sato, T.; Yashima, E. Hierarchical Amplification of Macromolecular Helicity in a Lyotropic Liquid Crystalline Charged Poly(phenylacetylene) by Nonracemic Dopants in Water and Its Helical Structure. Macromolecules 2006, 39, 5371-5380.

(S8) Shirakawa, H.; Ito, T.; Ikeda, S. Raman Scattering and Electronic Spectra of Poly(acetylene). Polym. J. 1973, 4, 460-462.

(S9) Tabata, M.; Tanaka, Y.; Sadahiro, Y.; Sone, T.; Yokota, K.; Miura, I. Pressure-Induced Cis to Trans Isomerization of Aromatic Polyacetylenes. 2. Poly((o-ethoxyphenyl)acetylene) Stereoregularly Polymerized Using a Rh Complex Catalyst. Macromolecules 1997, 30, 5200-5204.

(S10) Ohsawa, S.; Sakurai, S.-i.; Nagai, K.; Banno, M.; Maeda, K.; Kumaki, J.; Yashima, E. Hierarchical Amplification of Macromolecular Helicity of Dynamic Helical Poly(phenylacetylene)s Composed of Chiral and Achiral Phenylacetylenes in Dilute Solution, Liquid Crystal, and Two- 
Dimensional Crystal. J. Am. Chem. Soc. 2011, 133, 108-114.

(S11) Tang, Z.; Iida, H.; Hu, H.-Y.; Yashima, E. Remarkable Enhancement of the Enantioselectivity of an Organocatalyzed Asymmetric Henry Reaction Assisted by Helical Poly(phenylacetylene)s Bearing Cinchona Alkaloid Pendants via an Amide Linkage. ACS Macro Lett. 2012, 1, 261-265.

(S12) Goto, H.; Osawa, E. Viewpoint 11 - Approaches to the Global Minimum Problem. TheochemJ. Mol. Struct. 1993, 285, 157-168.

(S13) Goto, H.; Osawa, E. An Efficient Algorithm for Searching Low-Energy Conformers of Cyclic and Acyclic Molecules. J. Chem. Soc.-Perkin Trans. 2 1993, 187-198.

(S14) Grimme, S.; Antony, J.; Ehrlich, S.; Krieg, H. A Consistent and Accurate Ab Initio Parametrization of Density Functional Dispersion Correction (DFT-D) for the 94 Elements H-Pu. $J$. Chem. Phys. 2010, 132, 154104.

(S15) Gaussian 16, Revision C.01, M. J. Frisch, G. W. Trucks, H. B. Schlegel, G. E. Scuseria, M. A. Robb, J. R. Cheeseman, G. Scalmani, V. Barone, G. A. Petersson, H. Nakatsuji, X. Li, M. Caricato, A. V. Marenich, J. Bloino, B. G. Janesko, R. Gomperts, B. Mennucci, H. P. Hratchian, J. V. Ortiz, A. F. Izmaylov, J. L. Sonnenberg, D. Williams-Young, F. Ding, F. Lipparini, F. Egidi, J. Goings, B. Peng, A. Petrone, T. Henderson, D. Ranasinghe, V. G. Zakrzewski, J. Gao, N. Rega, G. Zheng, W. Liang, M. Hada, M. Ehara, K. Toyota, R. Fukuda, J. Hasegawa, M. Ishida, T. Nakajima, Y. Honda, O. Kitao, H. Nakai, T. Vreven, K. Throssell, J. A. Montgomery, Jr., J. E. Peralta, F. Ogliaro, M. J. Bearpark, J. J. Heyd, E. N. Brothers, K. N. Kudin, V. N. Staroverov, T. A. Keith, R. Kobayashi, J. Normand, K. Raghavachari, A. P. Rendell, J. C. Burant, S. S. Iyengar, J. Tomasi, M. Cossi, J. M. Millam, M. Klene, C. Adamo, R. Cammi, J. W. Ochterski, R. L. Martin, K. Morokuma, O. Farkas, J. B. Foresman, and D. J. Fox, Gaussian, Inc., Wallingford CT, 2016.

(S16) Rappé, A. K.; Casewit, C. J.; Colwell, K. S.; Goddard, W. A., III; Skiff, W. M. UFF, a Full Periodic Table Force Field for Molecular Mechanics and Molecular Dynamics Simulations. J. Am. Chem. Soc. 1992, 114, 10024-10035.

(S17) Casewit, C. J.; Colwell, K. S.; Rappé, A. K. Application of a Universal Force Field to Organic Molecules. J. Am. Chem. Soc. 1992, 114, 10035-10046.

(S18) Casewit, C. J.; Colwell, K. S.; Rappé, A. K. Application of a Universal Force Field to Main Group Compounds. J. Am. Chem. Soc. 1992, 114, 10046-10053.

(S19) Shimomura, K.; Ikai, T.; Kanoh, S.; Yashima, E.; Maeda, K. Switchable Enantioseparation Based on Macromolecular Memory of a Helical Polyacetylene in the Solid State. Nat. Chem. 2014, 
$6,429-434$.

(S20) Ishidate, R.; Okoshi, K.; Ikai, T.; Maeda, K.; Yashima, E., unpublished results.

(S21) Bai, B.; Zhu, H.-J.; Pan, W. Structure Influence of Chiral 1,1'-Biscarboline- $N, N^{\prime}$-Dioxide on the Enantioselective Allylation of Aldehydes with Allyltrichlorosilanes. Tetrahedron 2012, 68, 68296836.

(S22) Chelucci, G.; Murineddu, G.; Pinna, G. A. Chiral Pyridine N-Oxides: Useful Ligands for Asymmetric Catalysis. Tetrahedron: Asymmetry 2004, 15, 1373-1389.

(S23) Orito, Y.; Nakajima, M. Lewis Base Catalyzed Asymmetric Reactions Involving Hypervalent Silicate Intermediates. Synthesis 2006, 1391-1401.

(S24) Malkov, A. V.; Kočovský, P. Chiral N-Oxides in Asymmetric Catalysis. Eur. J. Org. Chem. 2007, 2007, 29-36.

(S25) Kotora, M. Synthesis of Axially Chiral Bipyridine $N, N$ '-Dioxides and Enantioselective Allylation of Aldehydes. Pure Appl. Chem. 2010, 82, 1813-1826.

(S26) Wrzeszcz, Z; Siedlecka, R. Heteroaromatic N-Oxides in Asymmetric Catalysis: A Review. Molecules 2020, 25, 330 .

(S27) Hrdina, R.; Opekar, F.; Roithová, J.; Kotora, M. Neutral and Ionic Reaction Mechanisms for the Allylation of Aldehydes by Bipyridinen $N, N^{\prime}$-Dioxides. Chem. Commun. 2009, 2314-2316.

(S28) Ducháčková, L.; Kadlčíková, A.; Kotora, M.; Roithová, J. Oxygen Superbases as Polar Binding Pockets in Nonpolar Solvents. J. Am. Chem. Soc. 2010, 132, 12660-12667.

(S29) Geng, C. H.; Zhu, R. X.; Zhang, D. J.; Lu, T. X.; Wheeler, S. E.; Liu, C. B. Solvent Dependence of the Stereoselectivity in Bipyridine $N, N^{\prime}$-Dioxide Catalyzed Allylation of Aromatic Aldehydes: A Computational Perspective. Mol. Catal. 2020, 483, 110712. 


\section{9. ${ }^{1} \mathrm{H}$ and ${ }^{13} \mathrm{C}$ NMR Spectral Data}

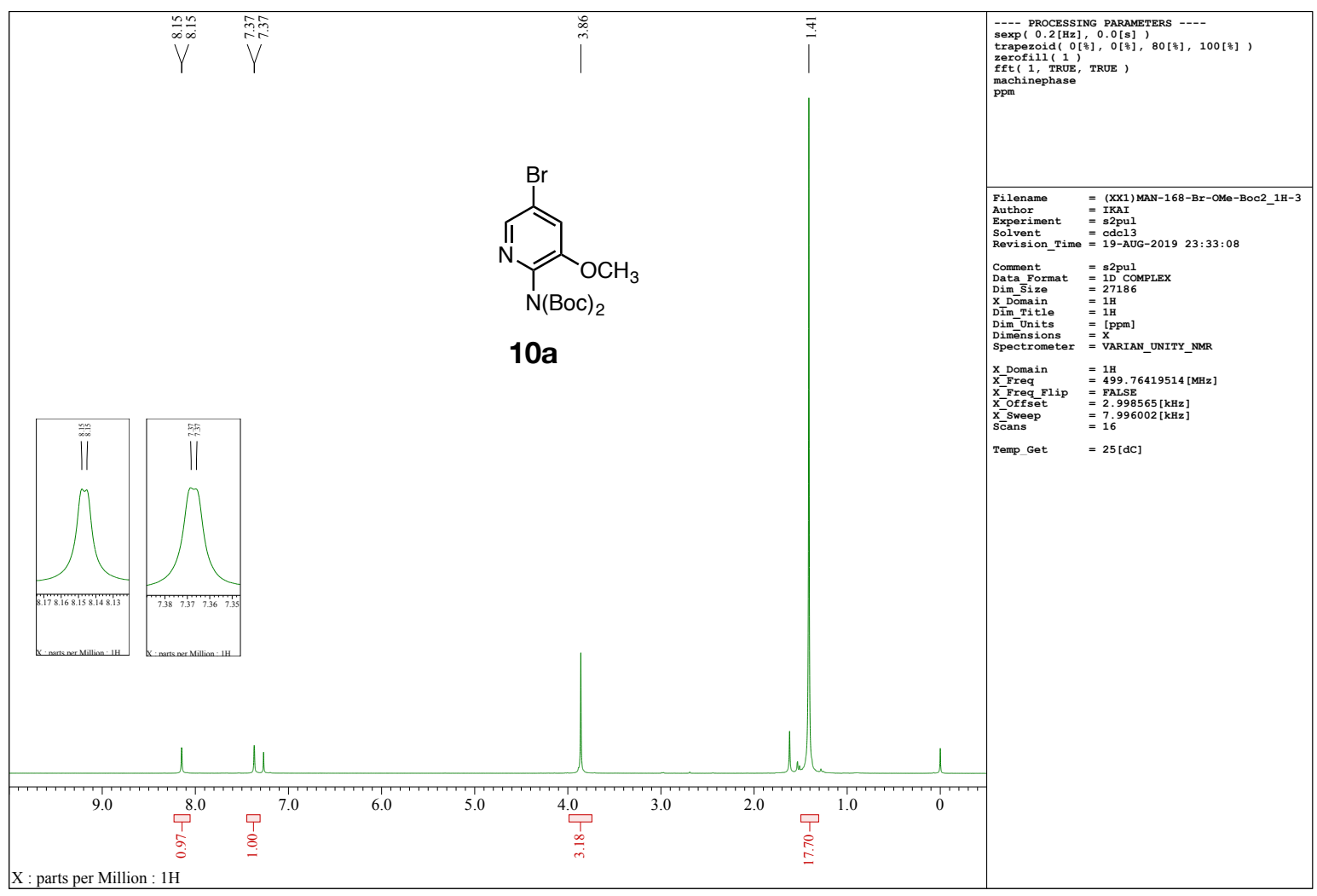

Figure S30. ${ }^{1} \mathrm{H}$ NMR $\left(500 \mathrm{MHz}, \mathrm{CDCl}_{3}, 25^{\circ} \mathrm{C}\right)$ spectrum of $\mathbf{1 0 a}$.

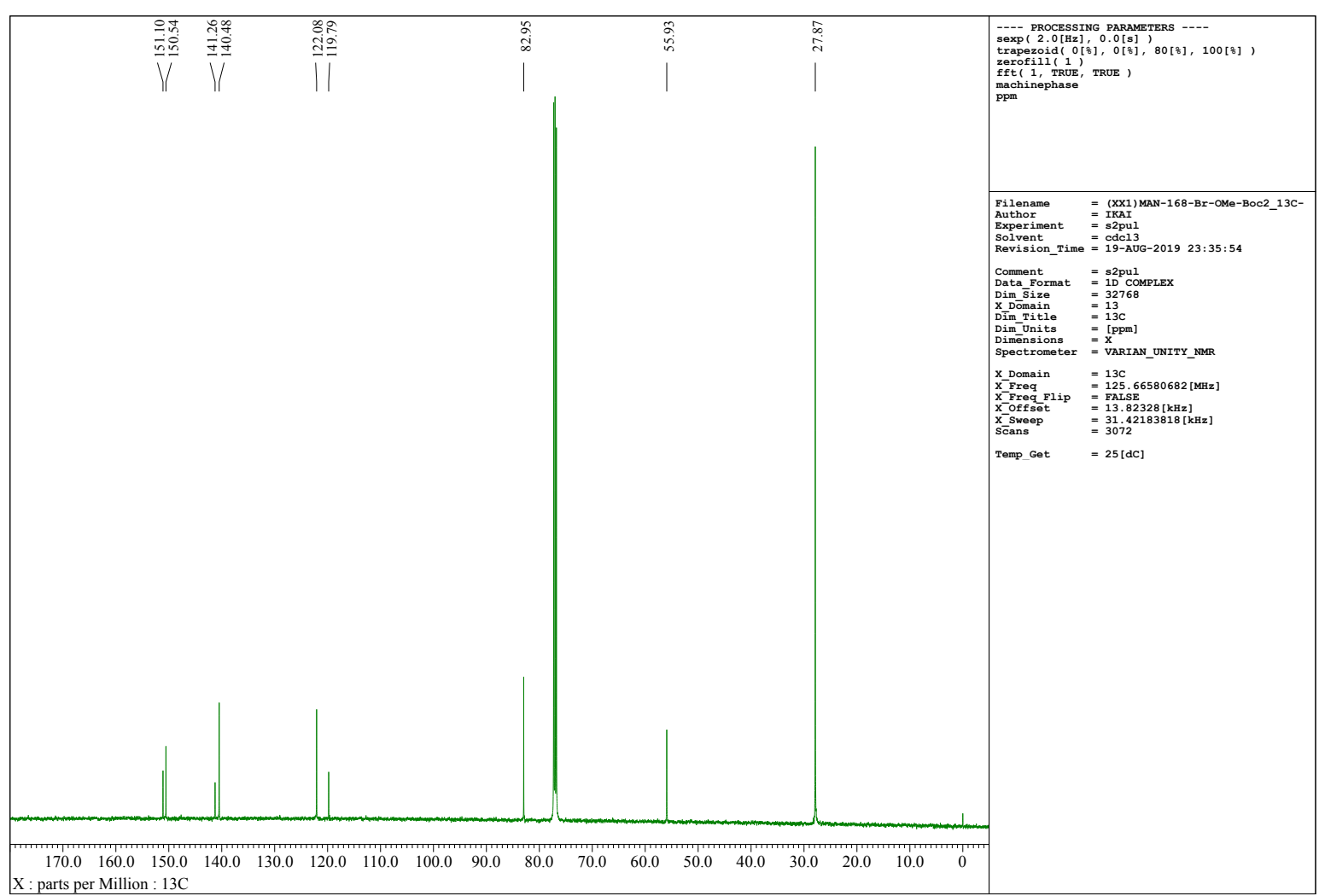

Figure S31. ${ }^{13} \mathrm{C}$ NMR $\left(126 \mathrm{MHz}, \mathrm{CDCl}_{3}, 25^{\circ} \mathrm{C}\right)$ spectrum of $\mathbf{1 0 a}$. 


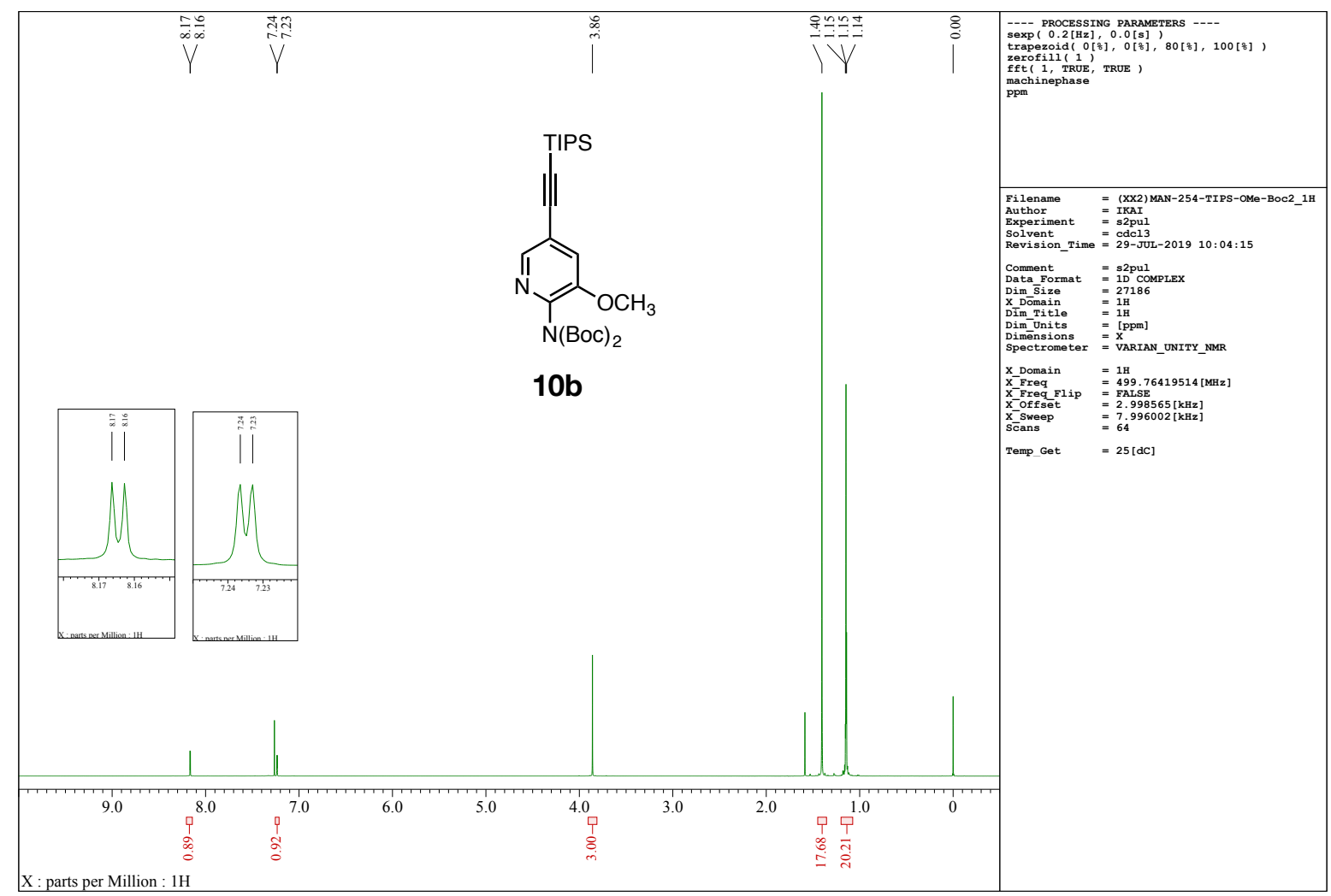

Figure S32. ${ }^{1} \mathrm{H} \mathrm{NMR}\left(500 \mathrm{MHz}, \mathrm{CDCl}_{3}, 25{ }^{\circ} \mathrm{C}\right)$ spectrum of $\mathbf{1 0 b}$.

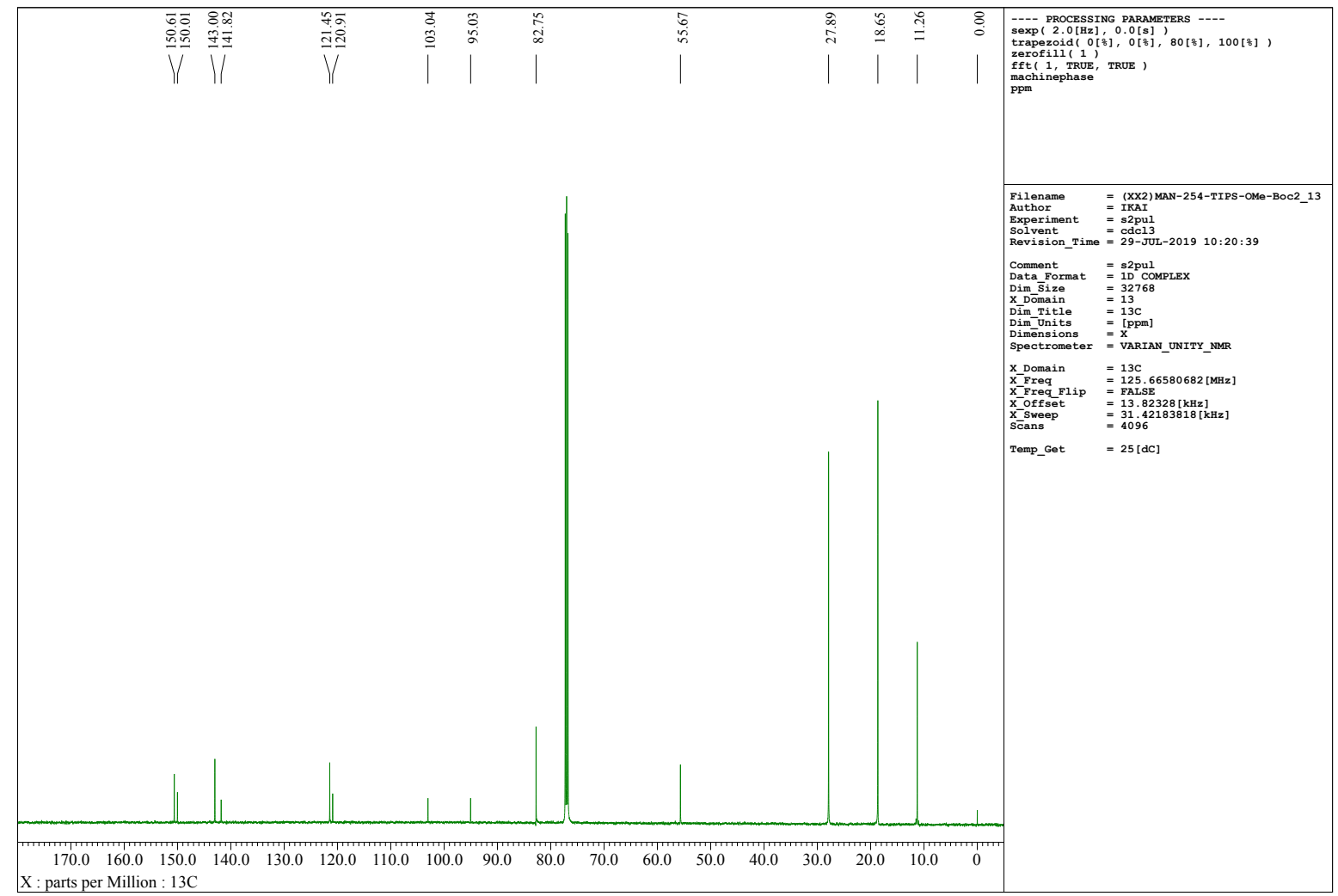

Figure S33. ${ }^{13} \mathrm{C} \mathrm{NMR}\left(126 \mathrm{MHz}, \mathrm{CDCl}_{3}, 25{ }^{\circ} \mathrm{C}\right)$ spectrum of $\mathbf{1 0 b}$. 


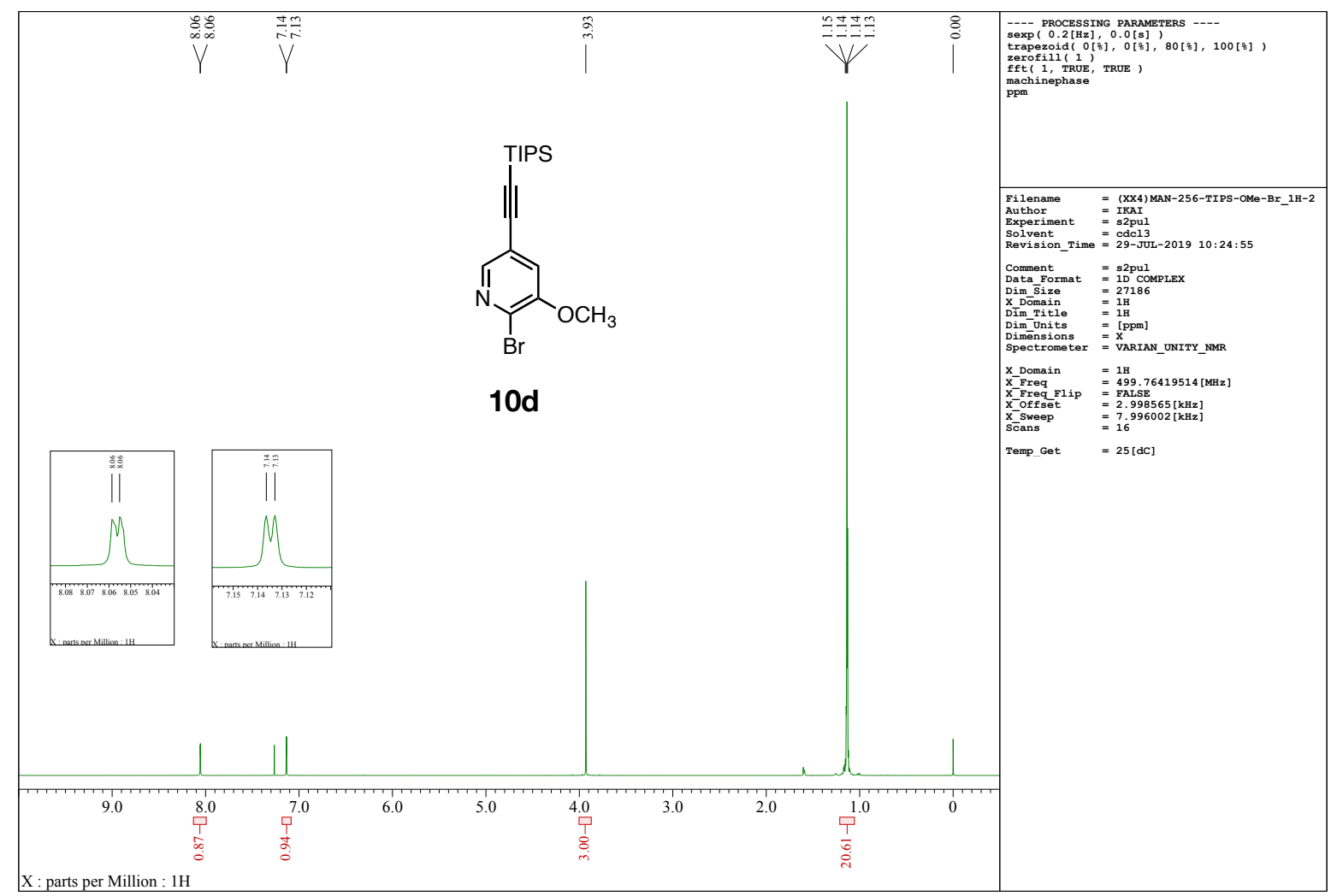

Figure S34. ${ }^{1} \mathrm{H} \mathrm{NMR}\left(500 \mathrm{MHz}, \mathrm{CDCl}_{3}, 25^{\circ} \mathrm{C}\right)$ spectrum of $\mathbf{1 0 d}$.

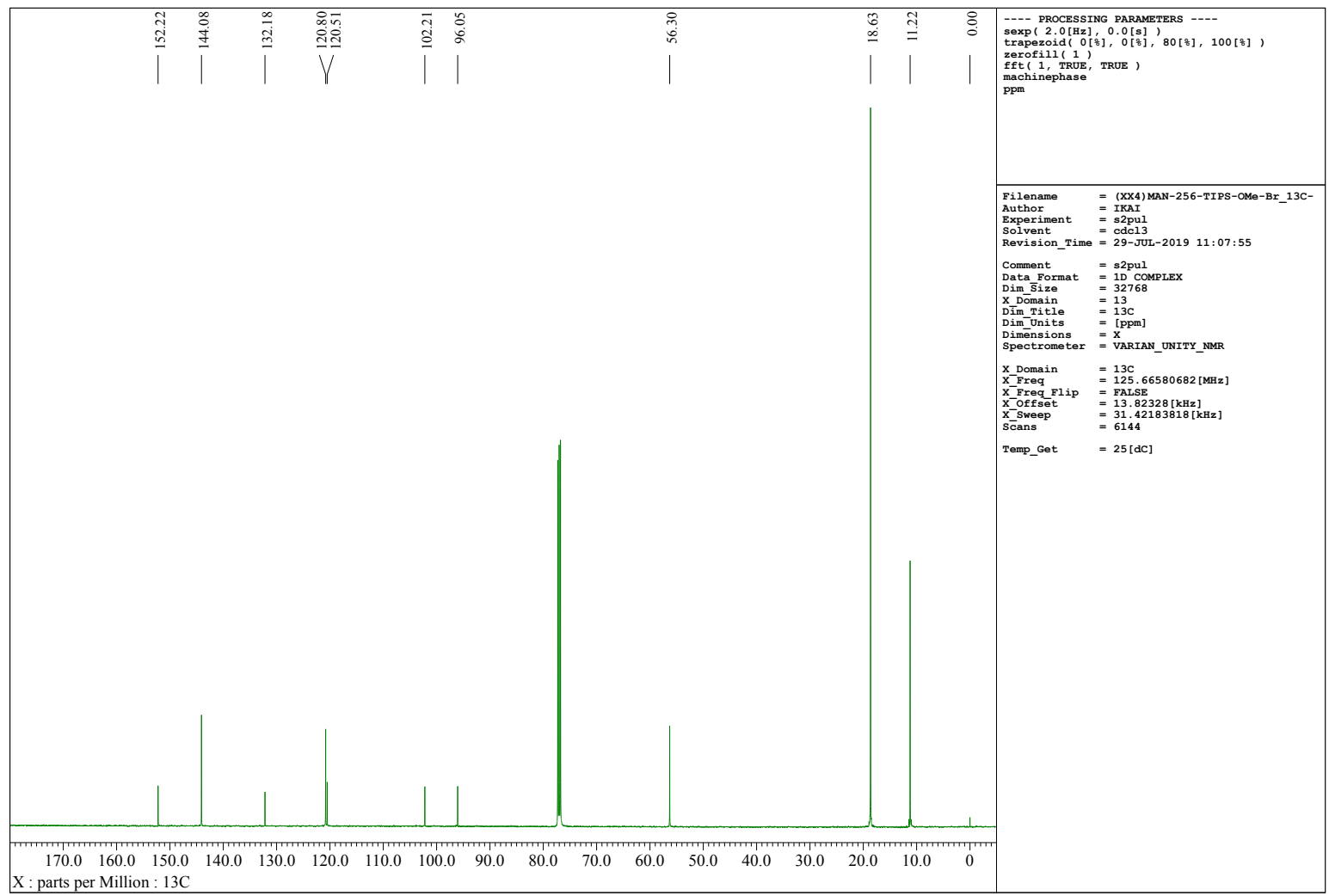

Figure S35. ${ }^{13} \mathrm{C} \mathrm{NMR}\left(126 \mathrm{MHz}, \mathrm{CDCl}_{3}, 25{ }^{\circ} \mathrm{C}\right)$ spectrum of $\mathbf{1 0 d}$. 


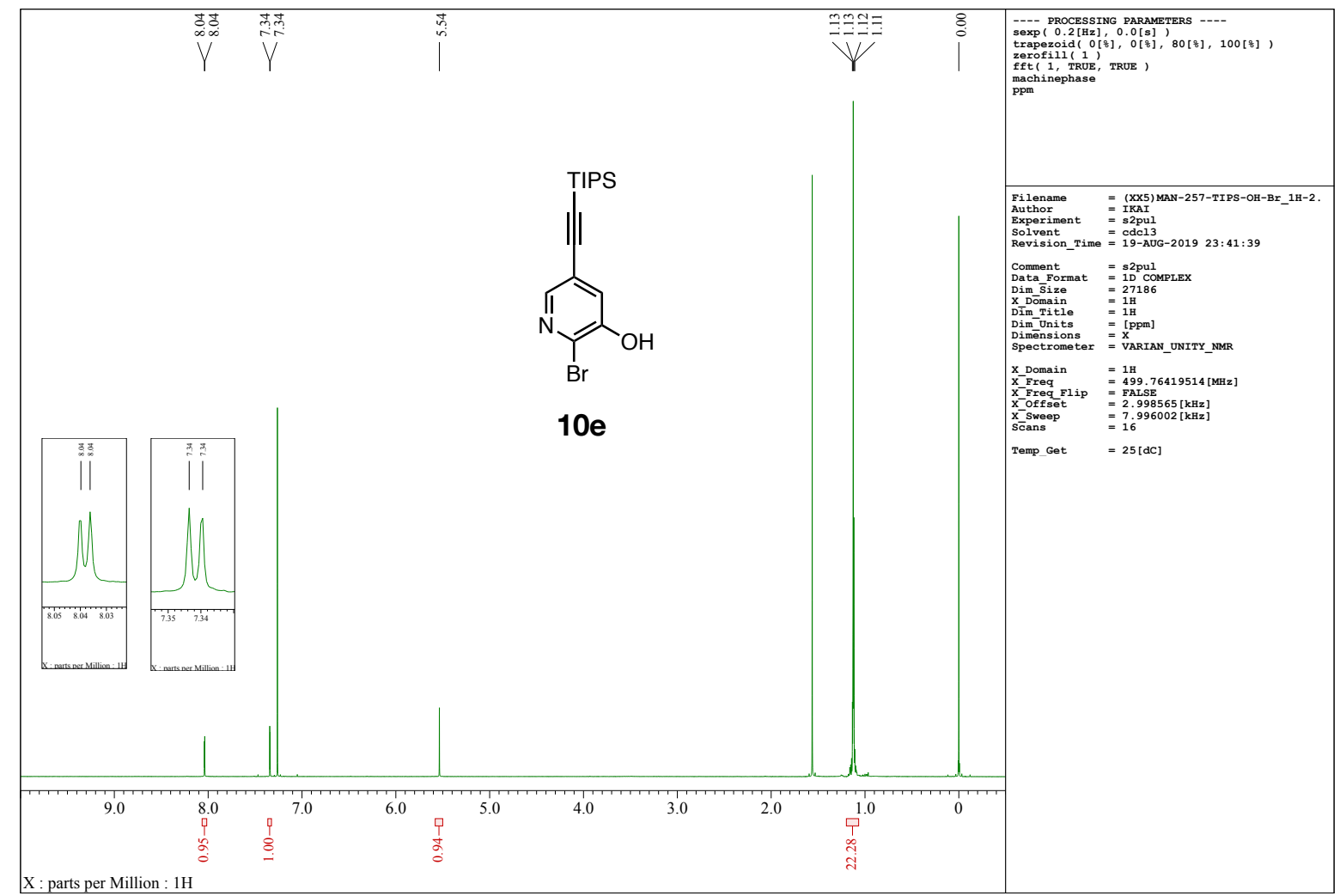

Figure S36. ${ }^{1} \mathrm{H} \mathrm{NMR}\left(500 \mathrm{MHz}, \mathrm{CDCl}_{3}, 25^{\circ} \mathrm{C}\right)$ spectrum of $\mathbf{1 0 e}$.

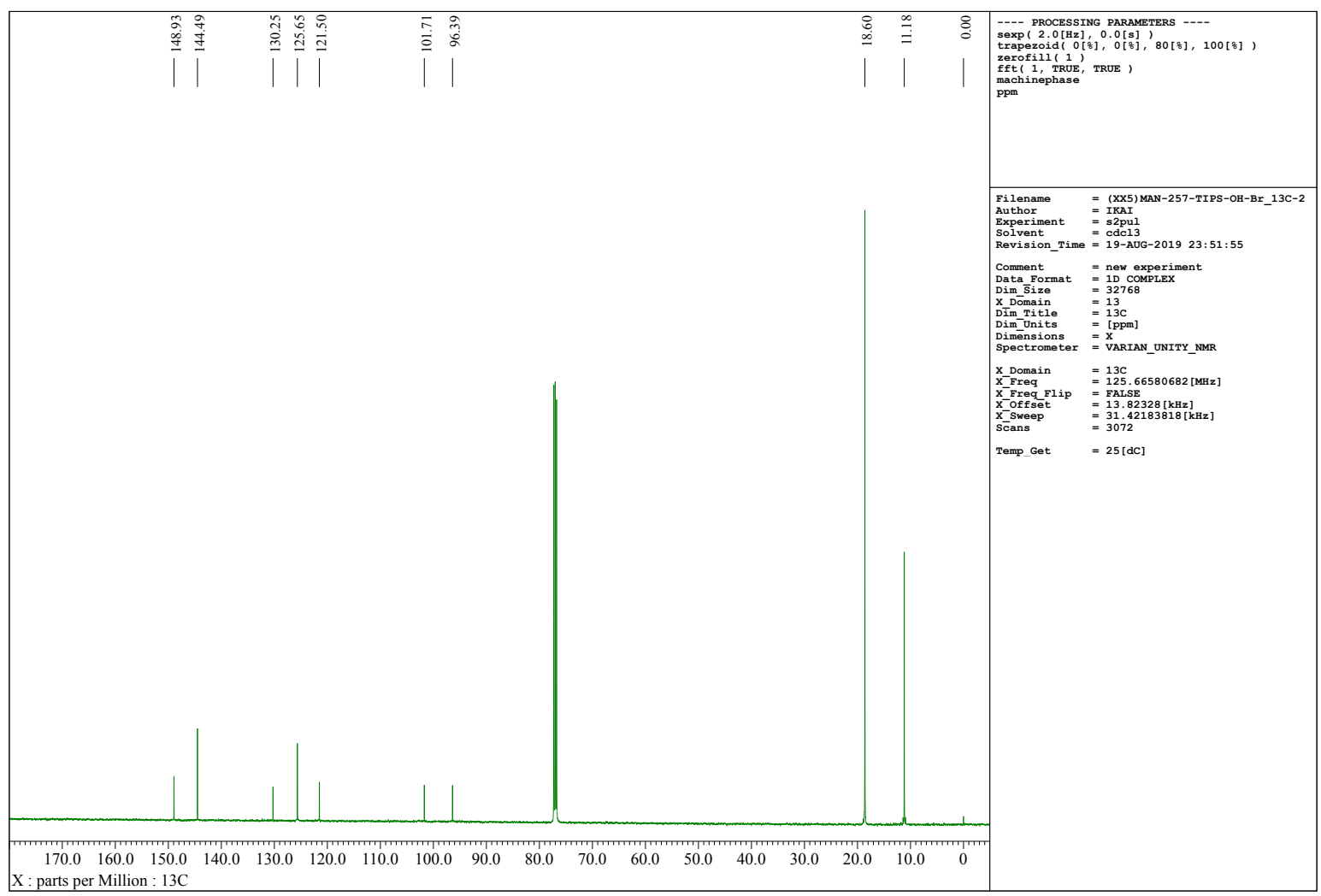

Figure S37. ${ }^{13} \mathrm{C}$ NMR $\left(126 \mathrm{MHz}, \mathrm{CDCl}_{3}, 25{ }^{\circ} \mathrm{C}\right)$ spectrum of $\mathbf{1 0 e}$. 


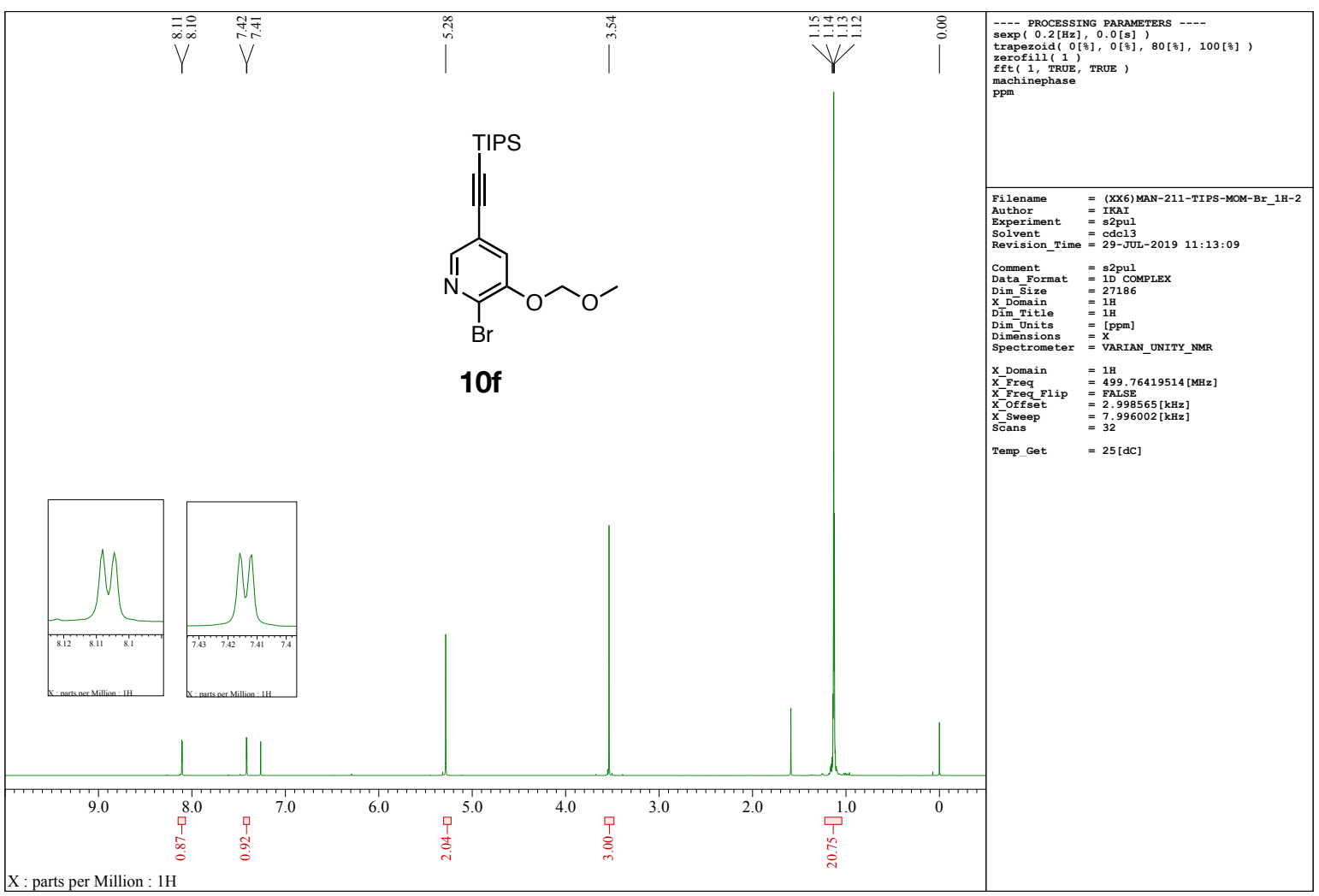

Figure S38. ${ }^{1} \mathrm{H} \mathrm{NMR}\left(500 \mathrm{MHz}, \mathrm{CDCl}_{3}, 25^{\circ} \mathrm{C}\right)$ spectrum of $\mathbf{1 0 f}$.

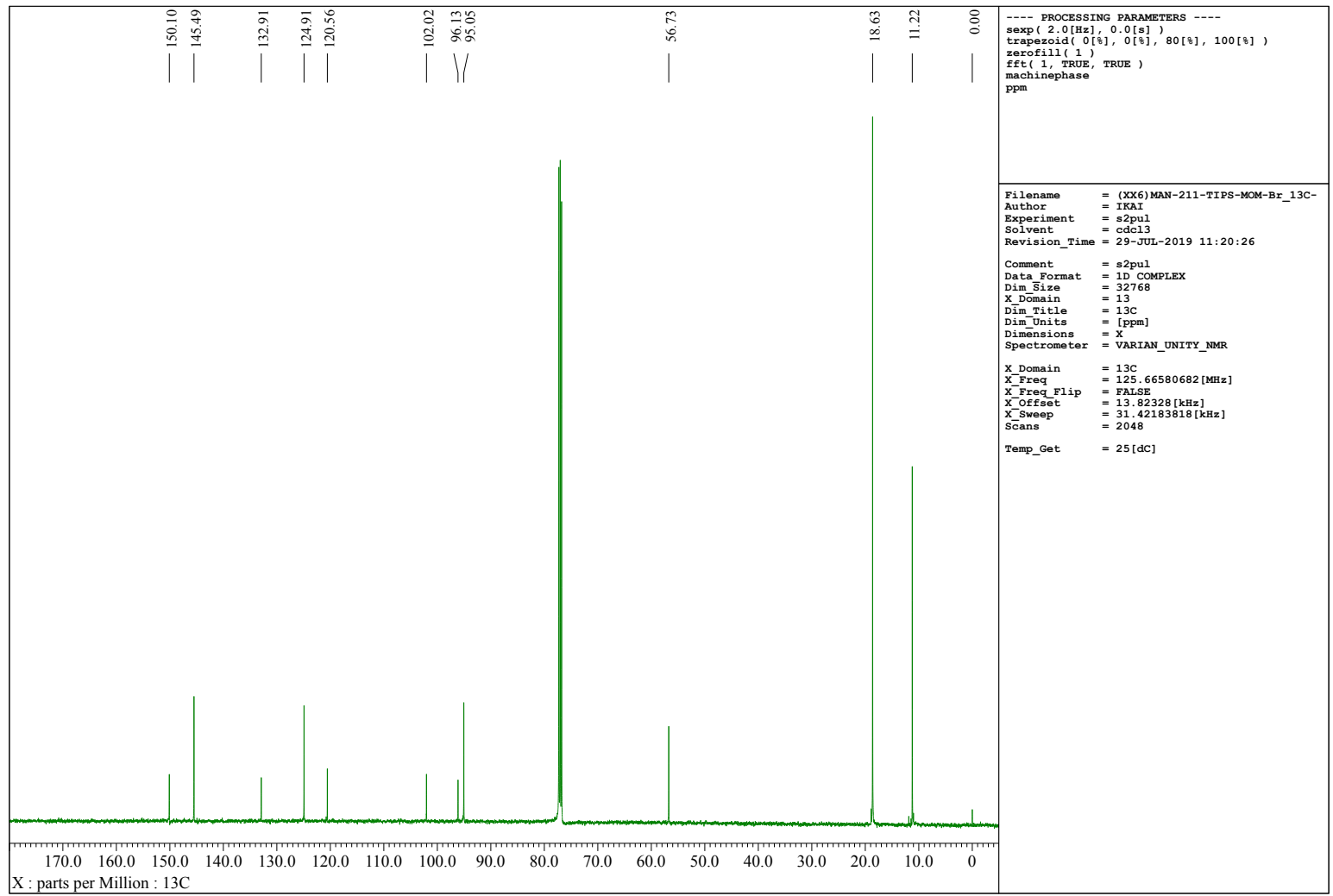

Figure S39. ${ }^{13} \mathrm{C} \mathrm{NMR}\left(126 \mathrm{MHz}, \mathrm{CDCl}_{3}, 25{ }^{\circ} \mathrm{C}\right)$ spectrum of $\mathbf{1 0 f}$. 


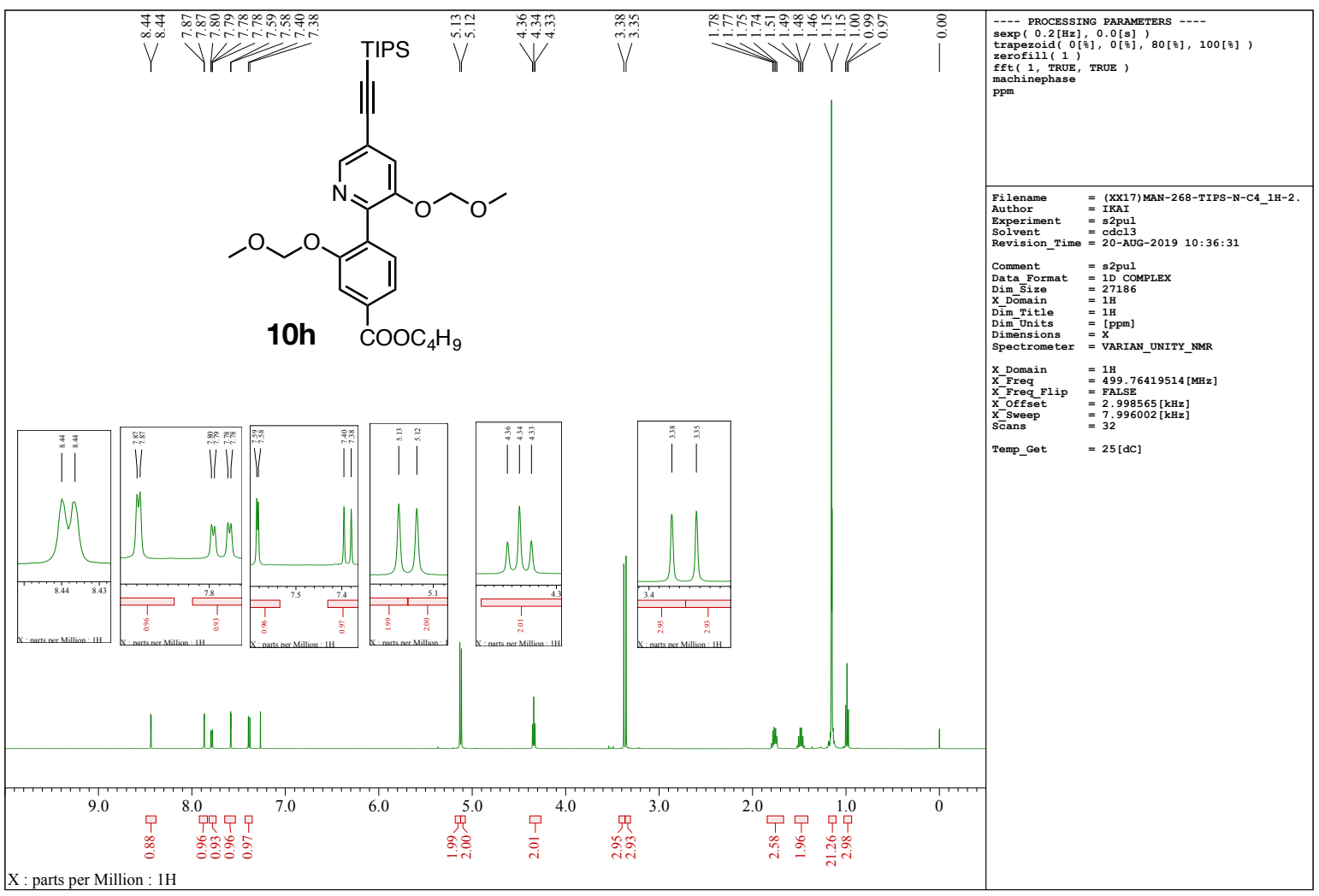

Figure S40. ${ }^{1} \mathrm{H} \mathrm{NMR}\left(500 \mathrm{MHz}, \mathrm{CDCl}_{3}, 25{ }^{\circ} \mathrm{C}\right)$ spectrum of $\mathbf{1 0 h}$.

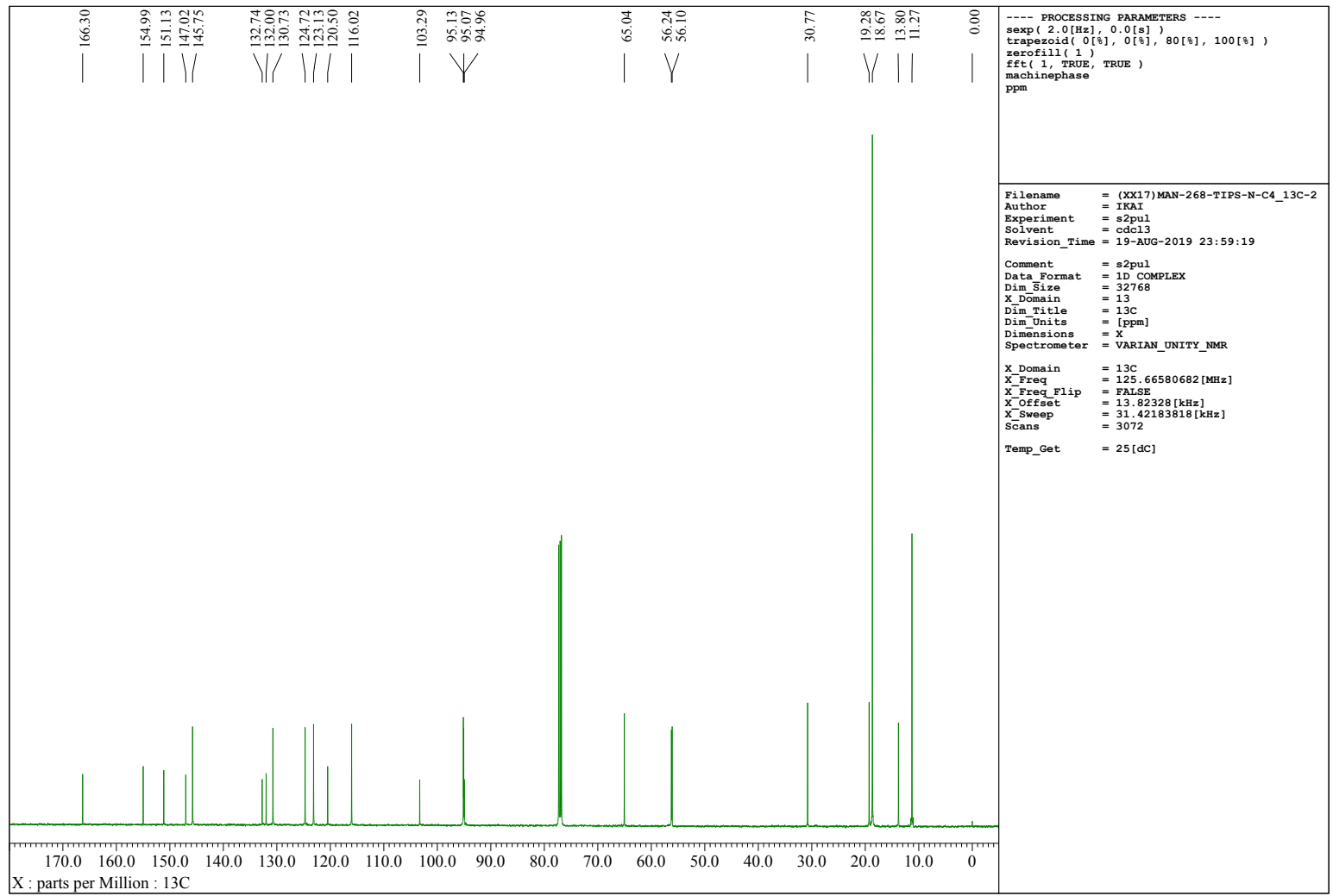

Figure S41. ${ }^{13} \mathrm{C} \mathrm{NMR}\left(126 \mathrm{MHz}, \mathrm{CDCl}_{3}, 25^{\circ} \mathrm{C}\right)$ spectrum of $\mathbf{1 0 h}$. 


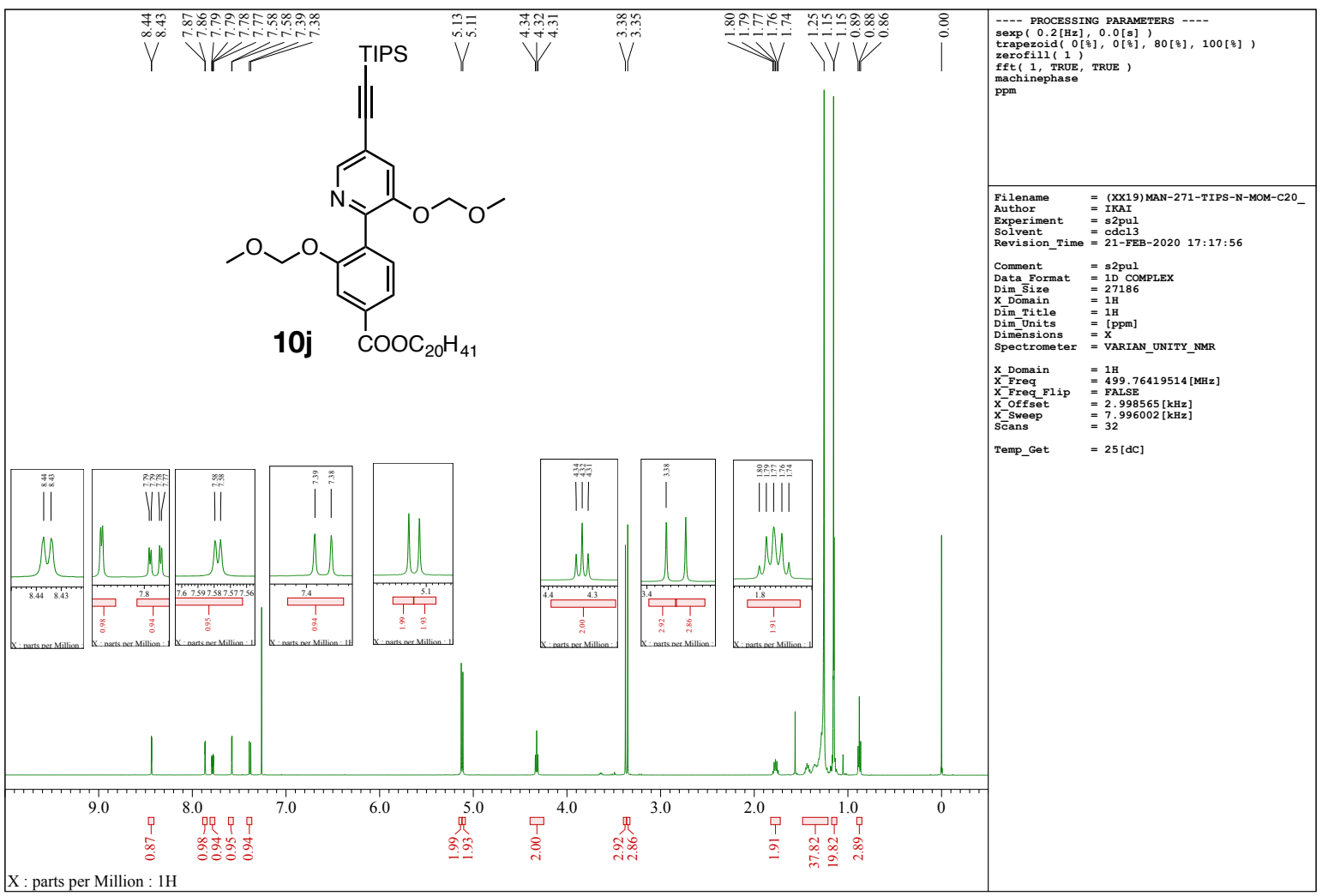

Figure S42. ${ }^{1} \mathrm{H} \mathrm{NMR}\left(500 \mathrm{MHz}, \mathrm{CDCl}_{3}, 25{ }^{\circ} \mathrm{C}\right)$ spectrum of $\mathbf{1 0 j}$.

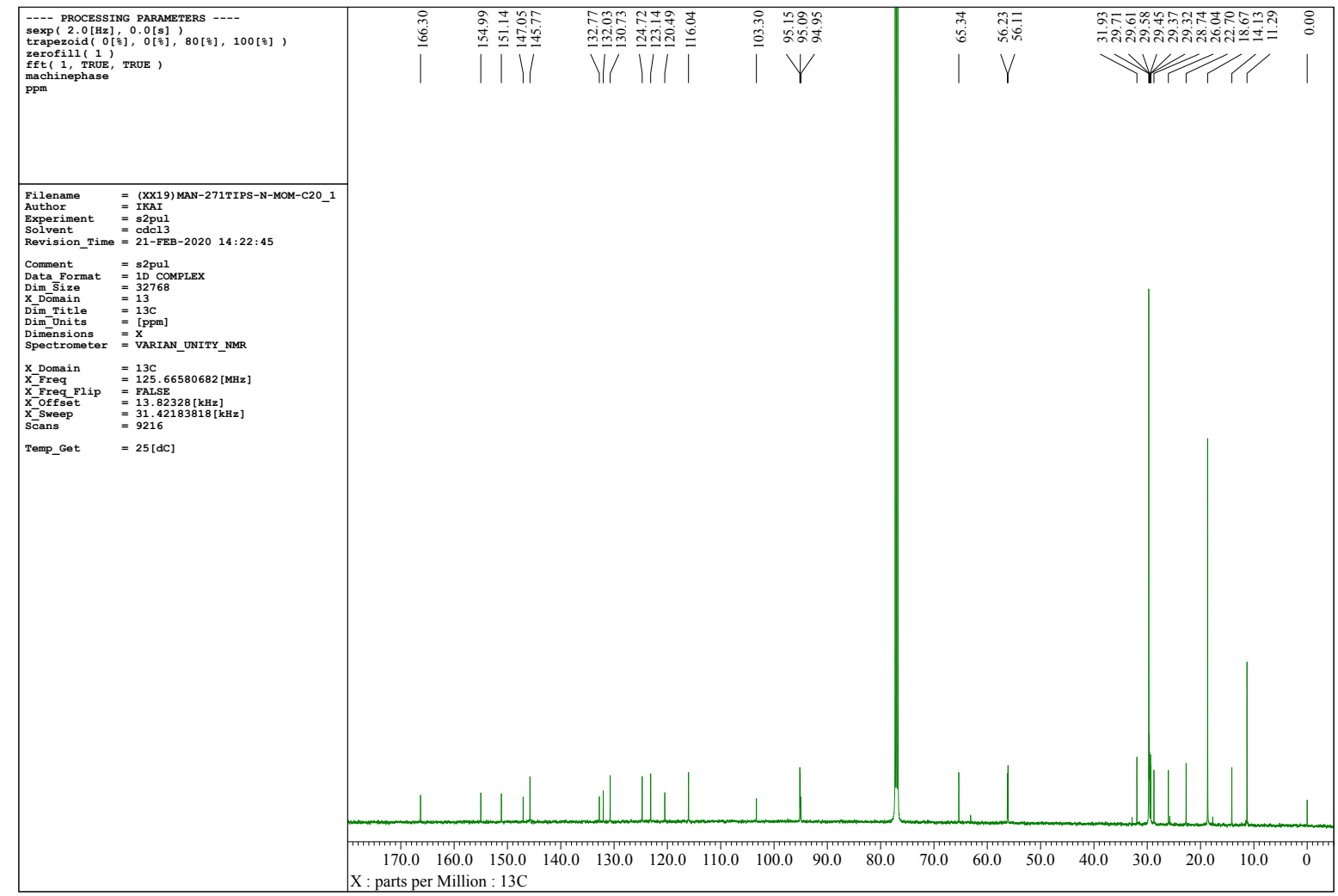

Figure S43. ${ }^{13} \mathrm{C}$ NMR $\left(126 \mathrm{MHz}, \mathrm{CDCl}_{3}, 25{ }^{\circ} \mathrm{C}\right)$ spectrum of $\mathbf{1 0 j}$. 


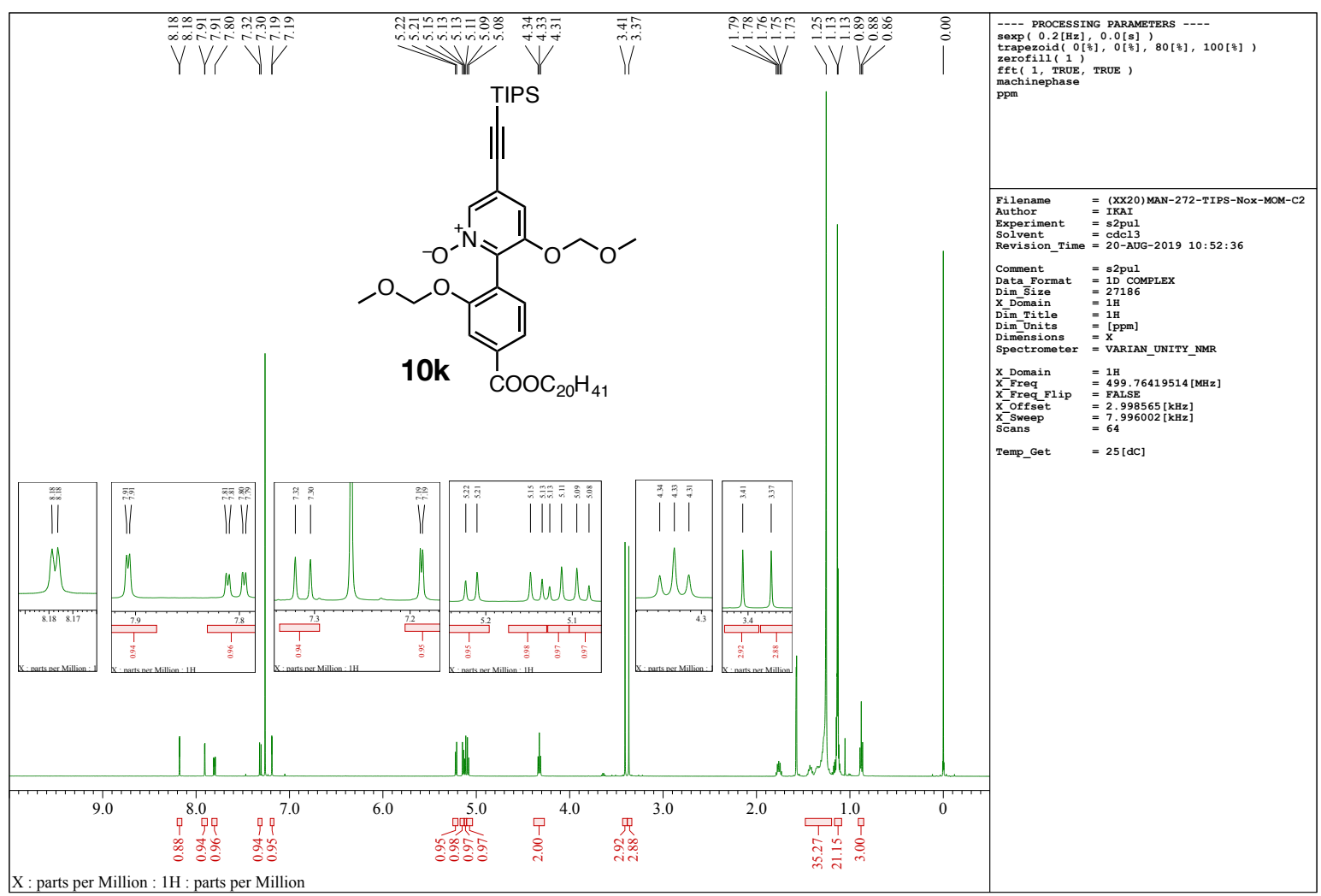

Figure S44. ${ }^{1} \mathrm{H} \mathrm{NMR}\left(500 \mathrm{MHz}, \mathrm{CDCl}_{3}, 25{ }^{\circ} \mathrm{C}\right)$ spectrum of $10 \mathrm{k}$.

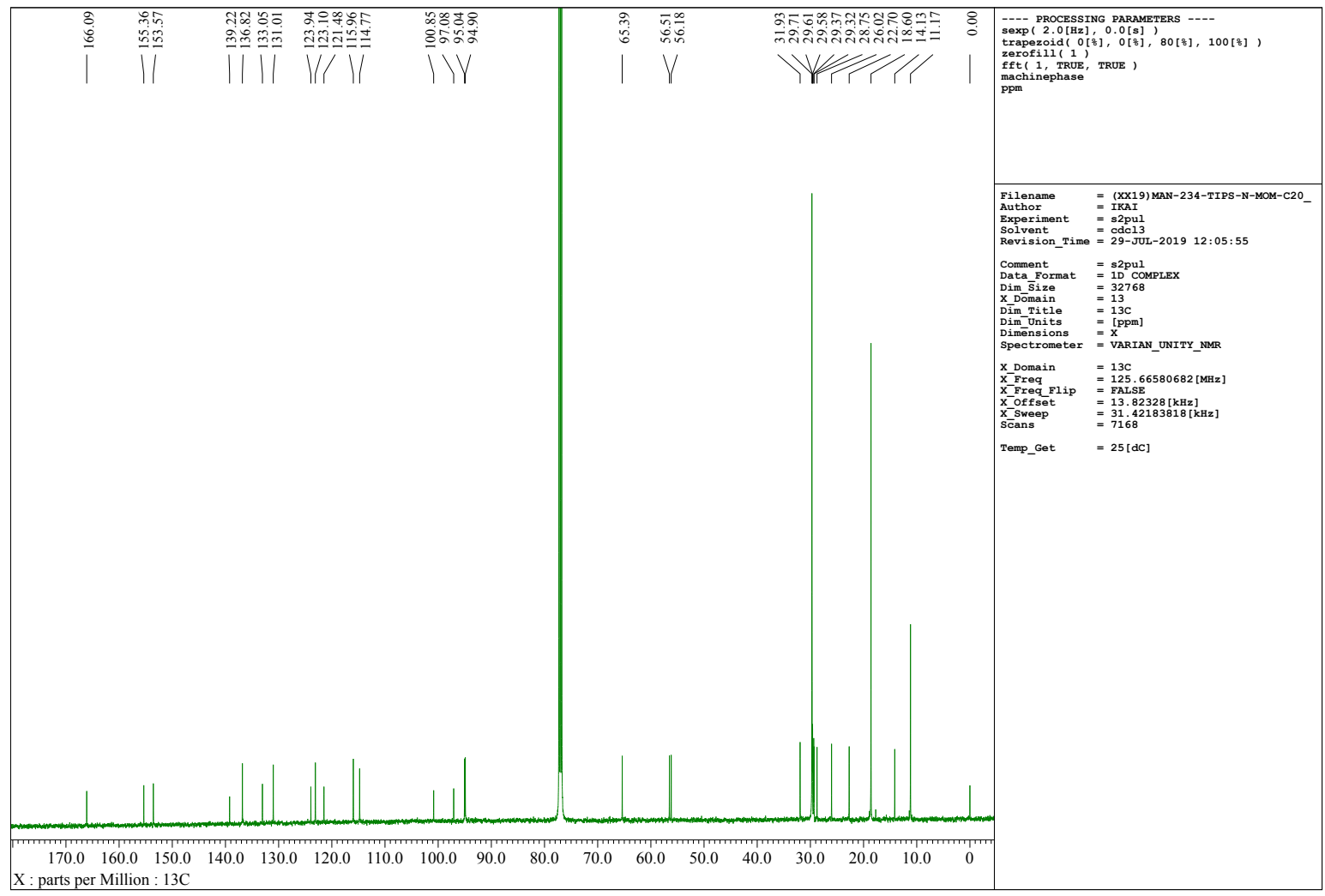

Figure $\mathbf{S 4 5} .{ }^{13} \mathrm{C} \mathrm{NMR}\left(126 \mathrm{MHz}, \mathrm{CDCl}_{3}, 25{ }^{\circ} \mathrm{C}\right)$ spectrum of $\mathbf{1 0 k}$. 


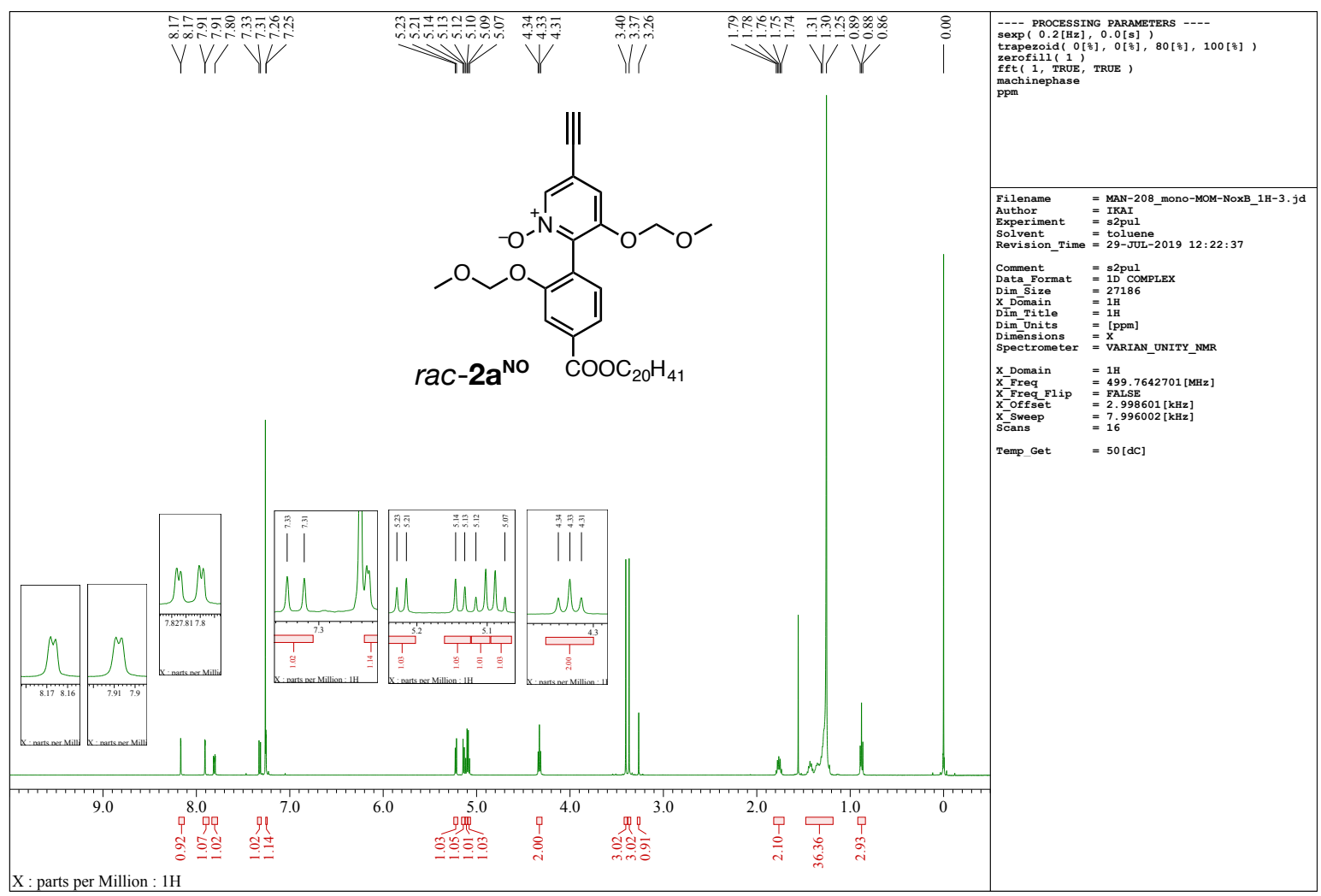

Figure S46. ${ }^{1} \mathrm{H}$ NMR $\left(500 \mathrm{MHz}, \mathrm{CDCl}_{3}, 50{ }^{\circ} \mathrm{C}\right)$ spectrum of $\mathrm{rac}-\mathbf{2 a}{ }^{\mathrm{NO}}$.

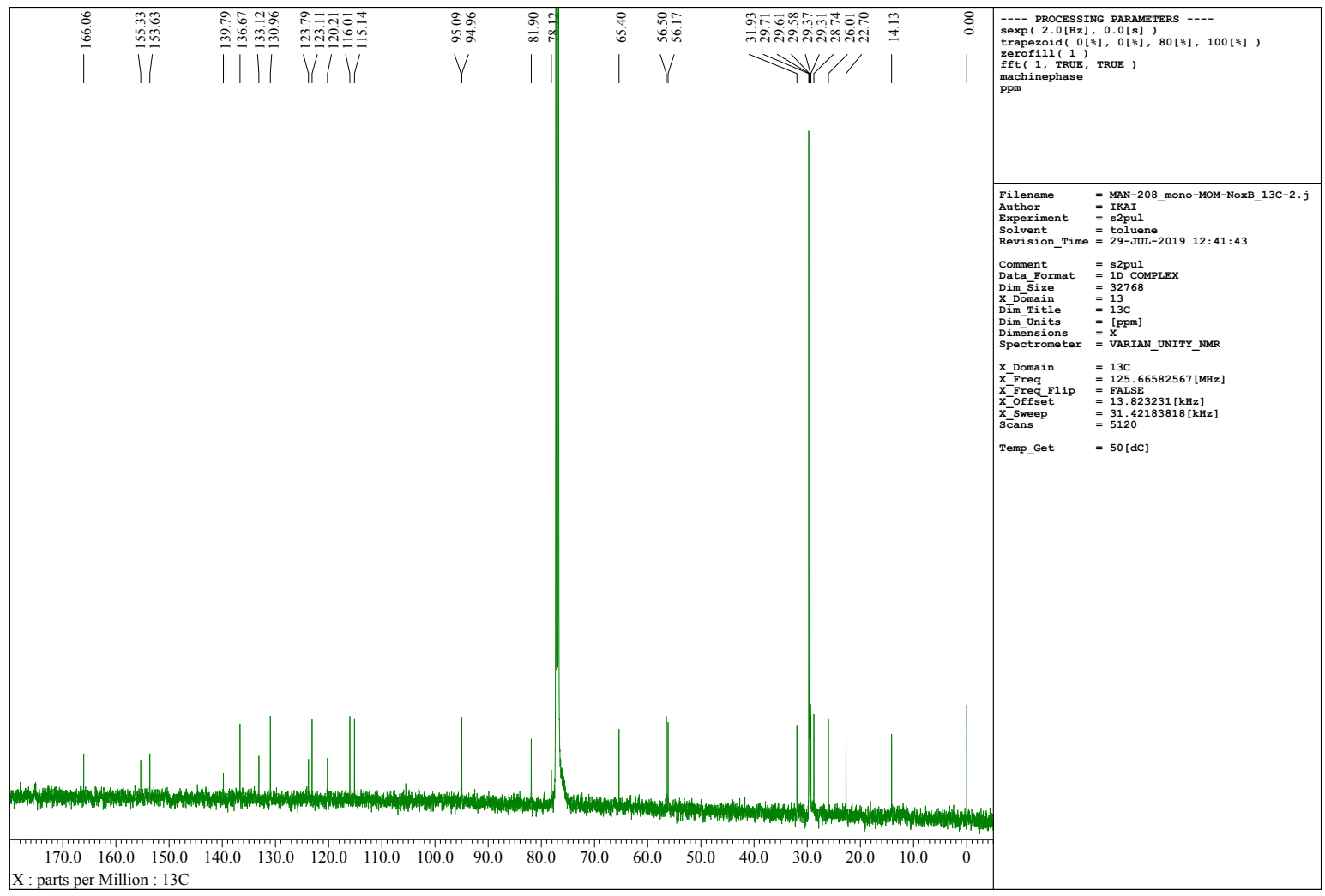

Figure S47. ${ }^{13} \mathrm{C} \mathrm{NMR}\left(126 \mathrm{MHz}, \mathrm{CDCl}_{3}, 50{ }^{\circ} \mathrm{C}\right)$ spectrum of $\mathrm{rac}-\mathbf{2} \mathbf{a}^{\mathrm{NO}}$. 


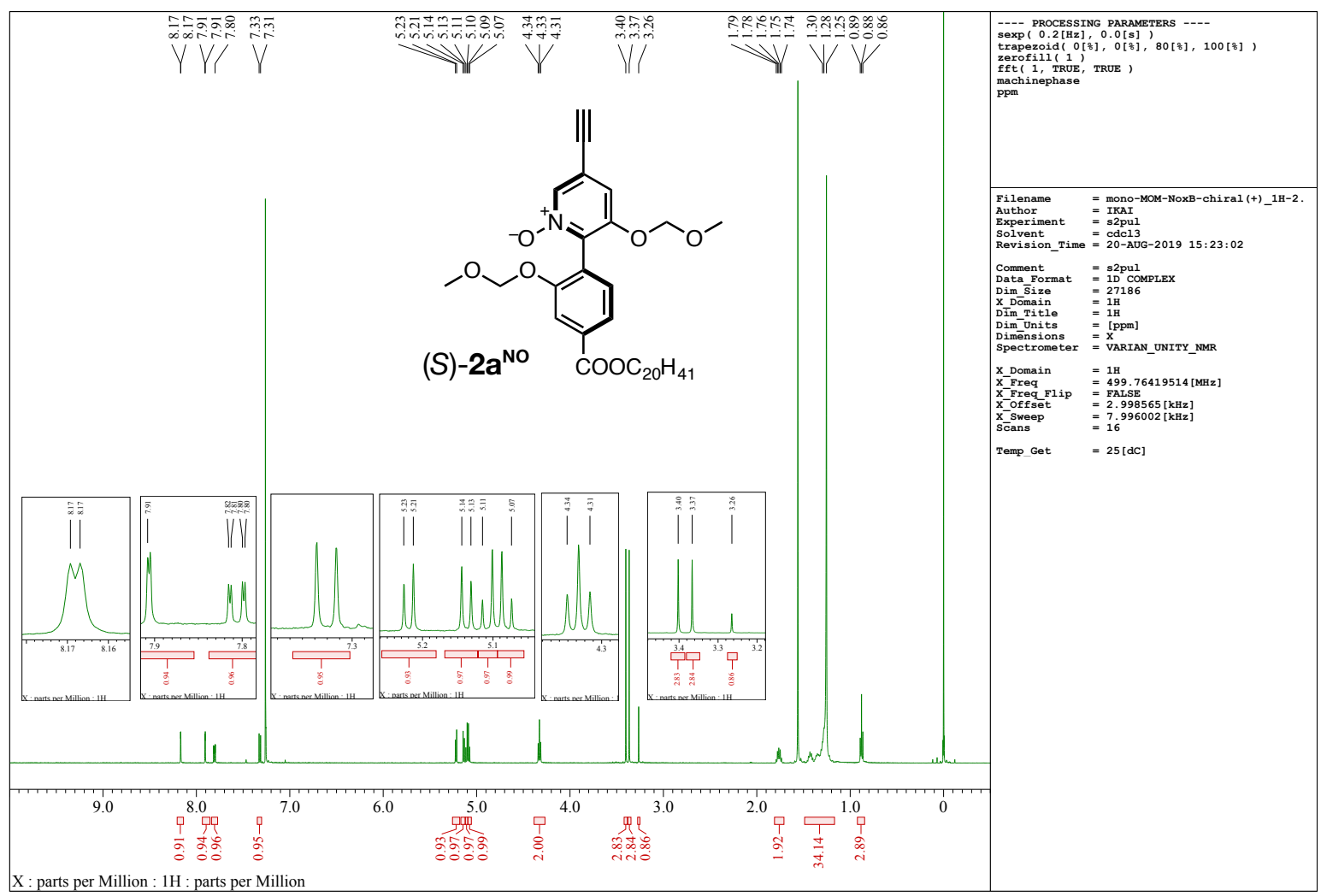

Figure S48. ${ }^{1} \mathrm{H}$ NMR $\left(500 \mathrm{MHz}, \mathrm{CDCl}_{3}, 25^{\circ} \mathrm{C}\right)$ spectrum of $(S)-\mathbf{2} \mathbf{a}^{\mathrm{NO}}$.

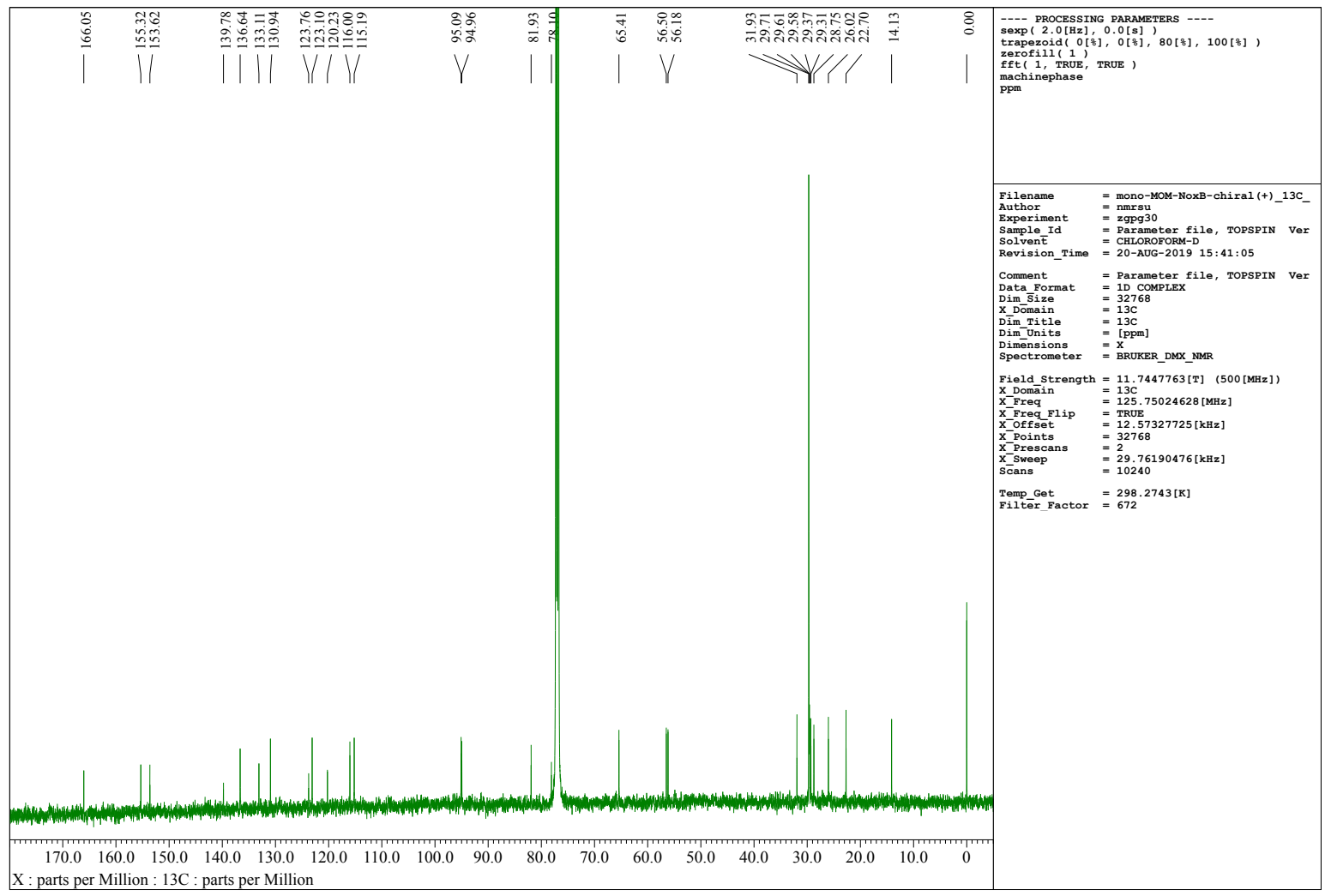

Figure S49. ${ }^{13} \mathrm{C} \mathrm{NMR}\left(126 \mathrm{MHz}, \mathrm{CDCl}_{3}, 25{ }^{\circ} \mathrm{C}\right) \operatorname{spectrum}$ of $(S)-\mathbf{2} \mathbf{a}^{\mathrm{NO}}$. 


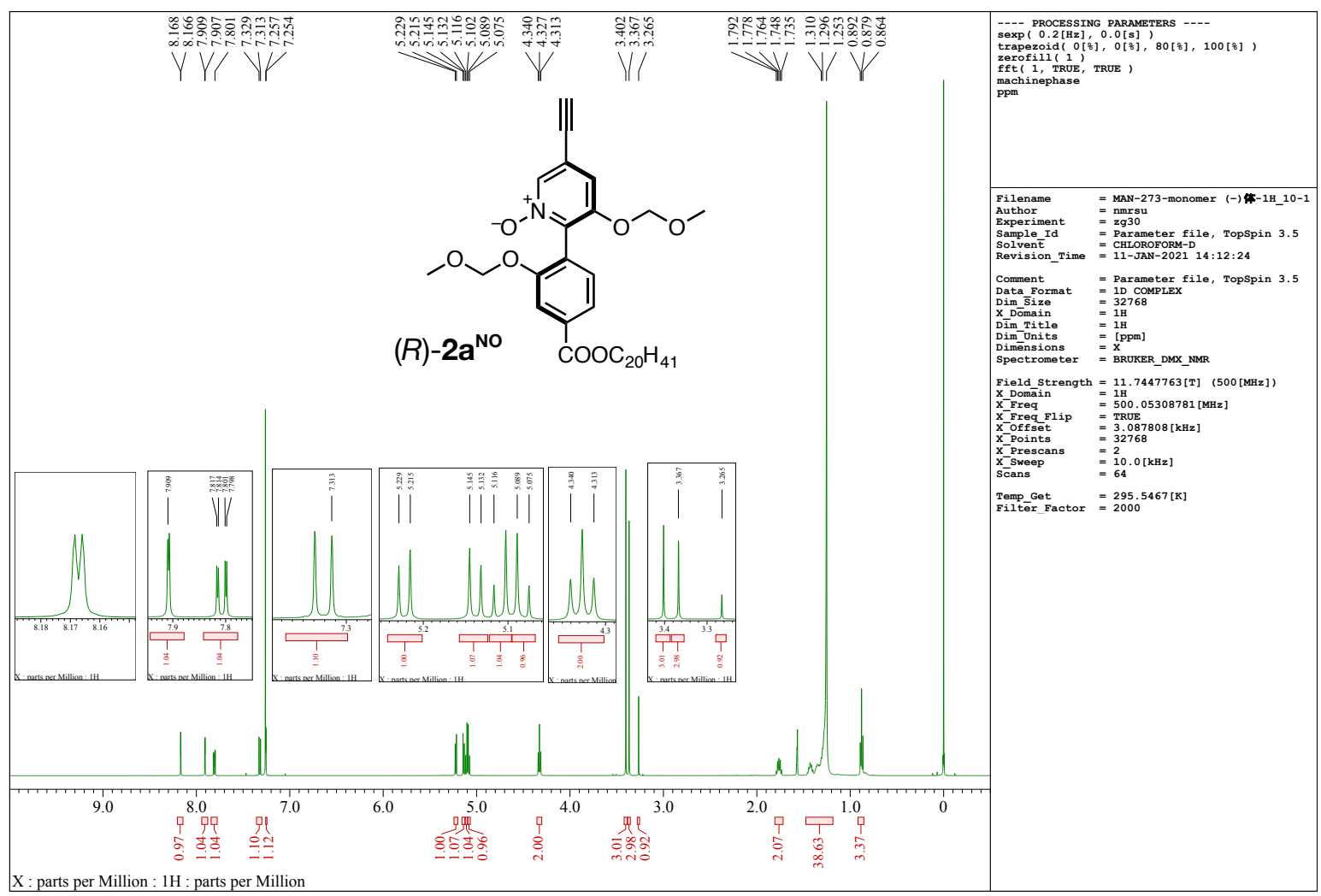

Figure S50. ${ }^{1} \mathrm{H}$ NMR $\left(500 \mathrm{MHz}, \mathrm{CDCl}_{3}, \mathrm{rt}\right)$ spectrum of $(R)-\mathbf{2} \mathbf{a}^{\mathrm{NO}}$.

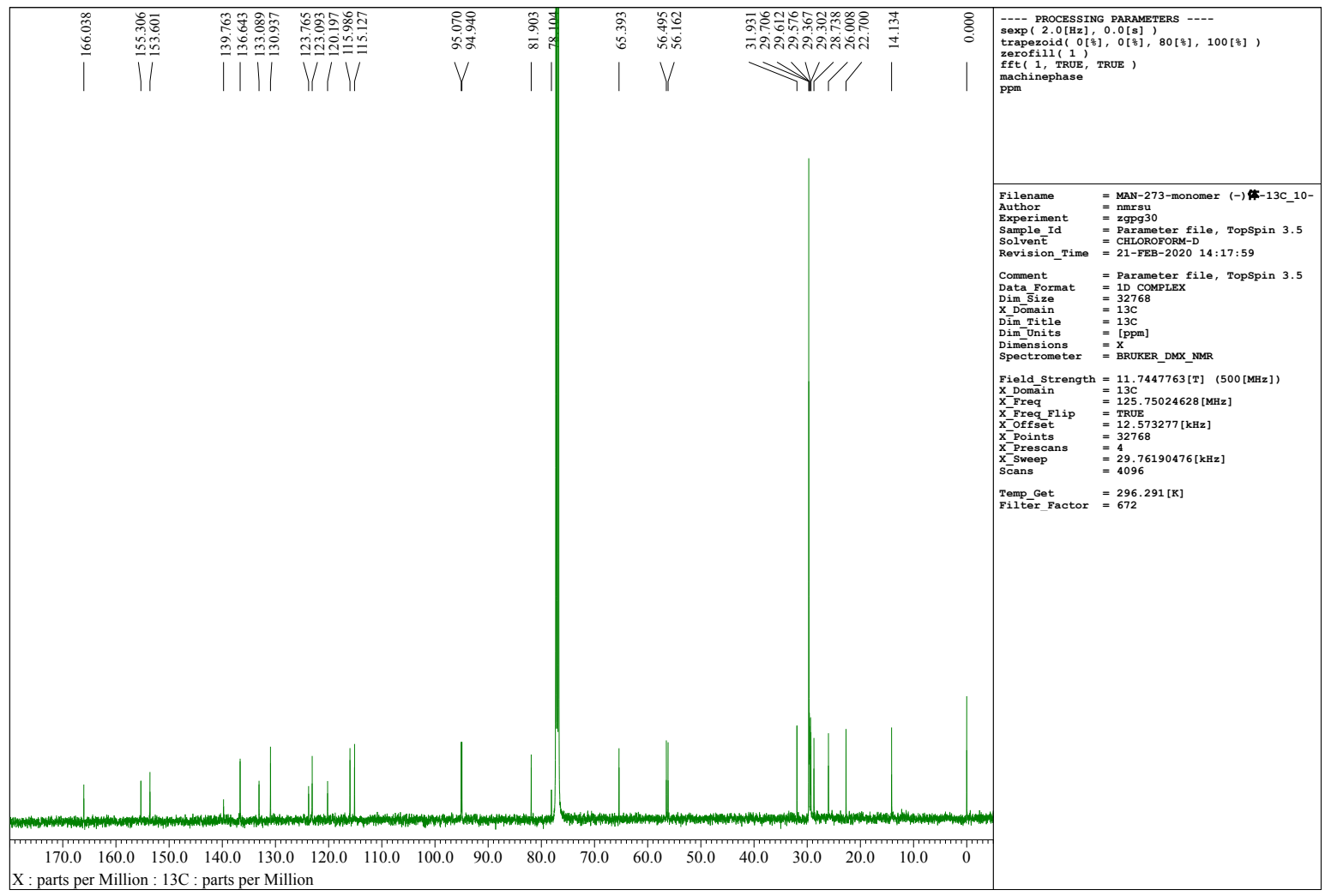

Figure S51. ${ }^{13} \mathrm{C} \mathrm{NMR}\left(126 \mathrm{MHz}, \mathrm{CDCl}_{3}, \mathrm{rt}\right)$ spectrum of $(R)-\mathbf{2} \mathbf{a}^{\mathrm{No}}$. 


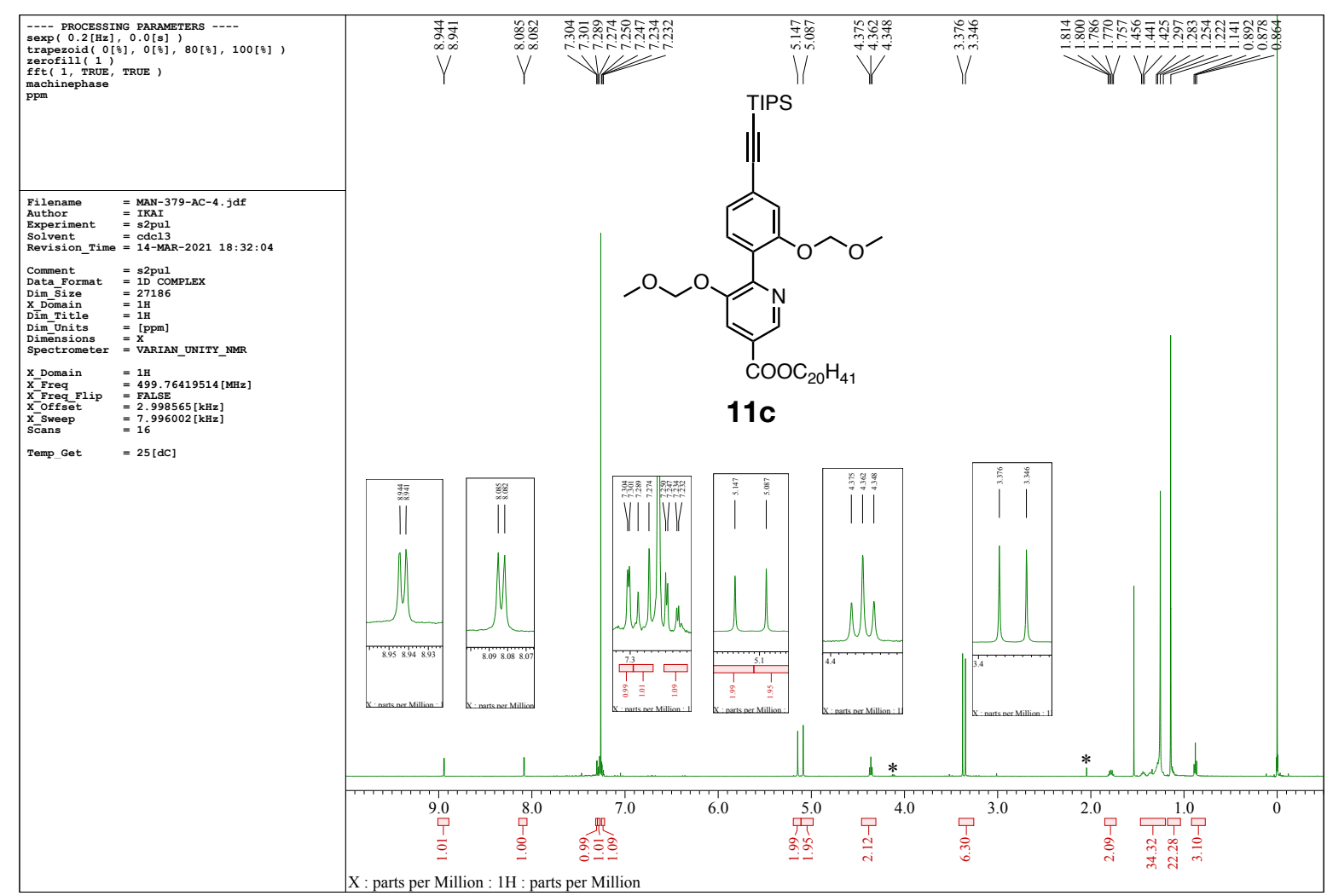

Figure S52. ${ }^{1} \mathrm{H}$ NMR $\left(500 \mathrm{MHz}, \mathrm{CDCl}_{3}, 25^{\circ} \mathrm{C}\right)$ spectrum of 11c. $*$ denotes the protons from residual ethyl acetate.

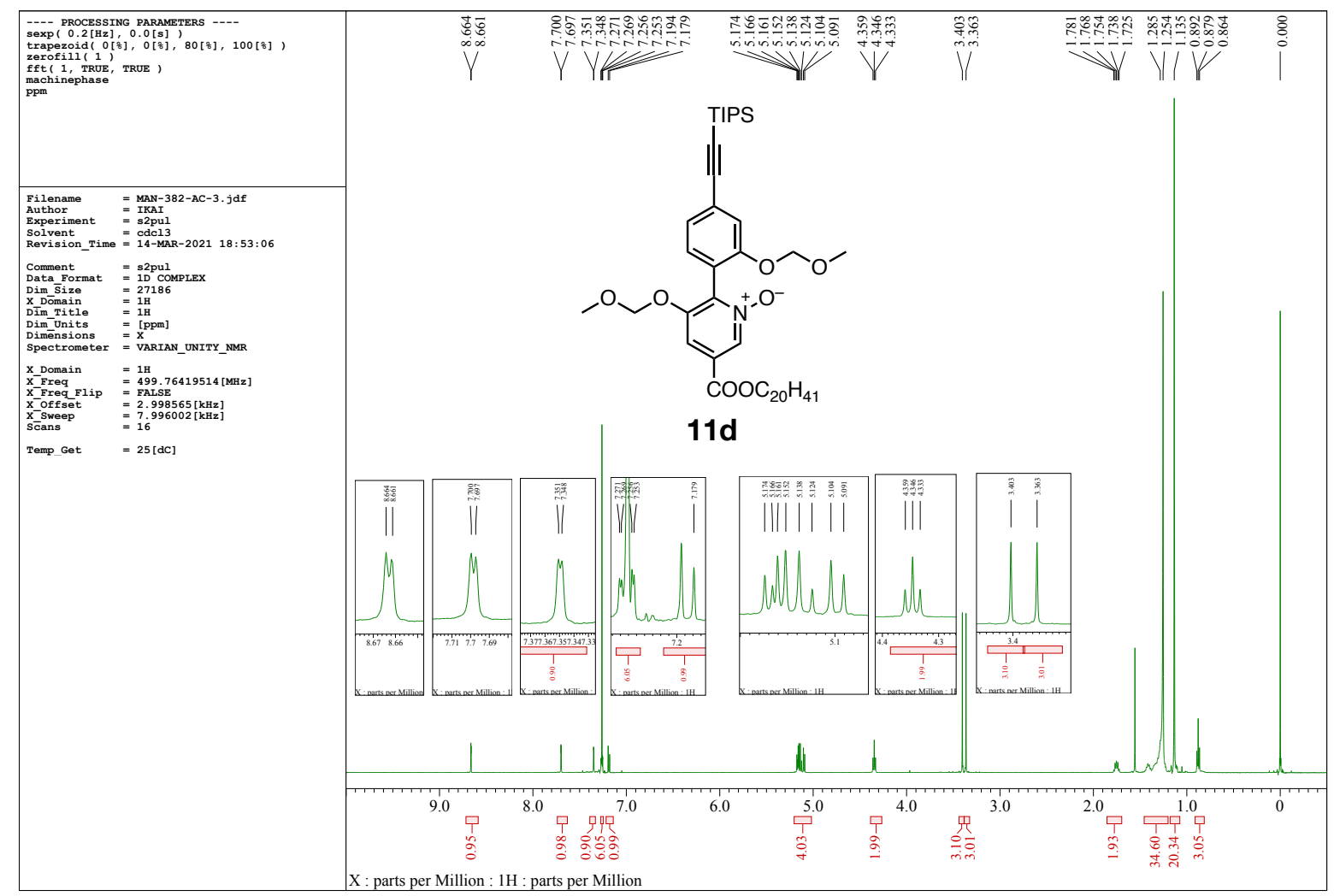

Figure S53. ${ }^{1} \mathrm{H}$ NMR $\left(500 \mathrm{MHz}, \mathrm{CDCl}_{3}, 25^{\circ} \mathrm{C}\right)$ spectrum of $11 d$. 


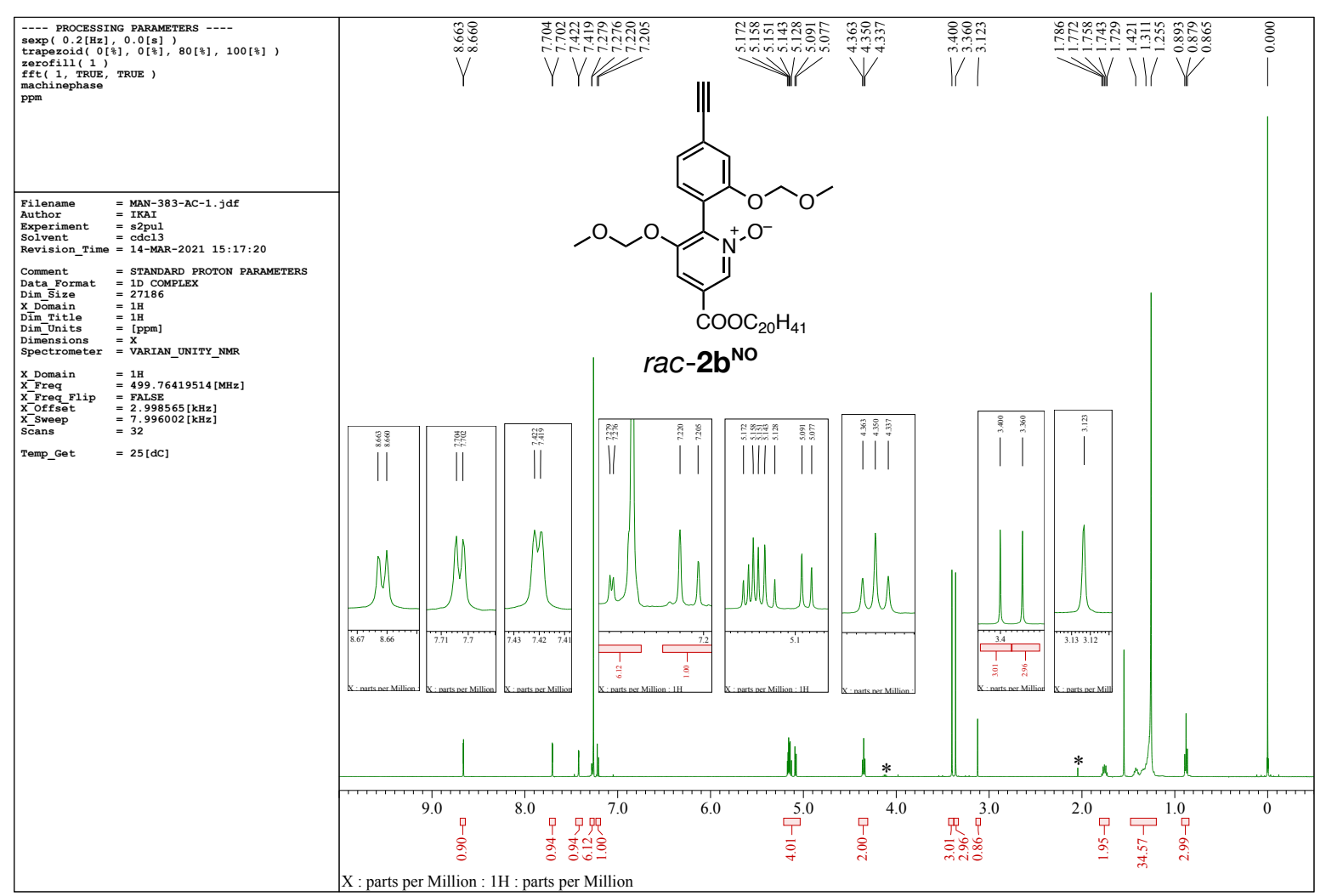

Figure S54. ${ }^{1} \mathrm{H} \mathrm{NMR}\left(500 \mathrm{MHz}, \mathrm{CDCl}_{3}, 25^{\circ} \mathrm{C}\right)$ spectrum of $\mathrm{rac}-\mathbf{2} \mathbf{b}^{\mathrm{NO}} . *$ denotes the protons from residual ethyl acetate.

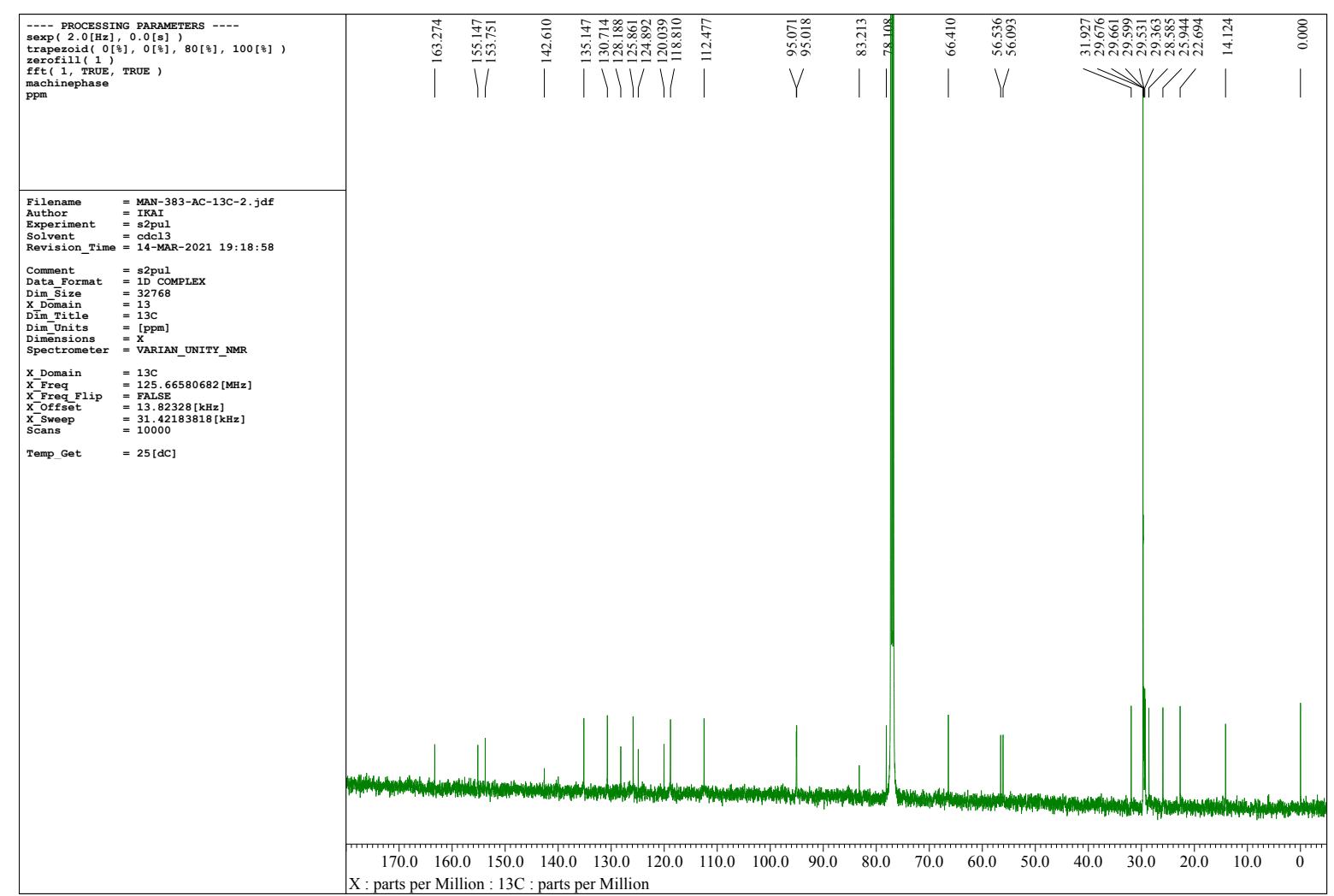

Figure S55. ${ }^{13} \mathrm{C} \mathrm{NMR}\left(126 \mathrm{MHz}, \mathrm{CDCl}_{3}, 25{ }^{\circ} \mathrm{C}\right)$ spectrum of $\mathrm{rac}-\mathbf{2} \mathbf{b}^{\mathrm{NO}}$. 


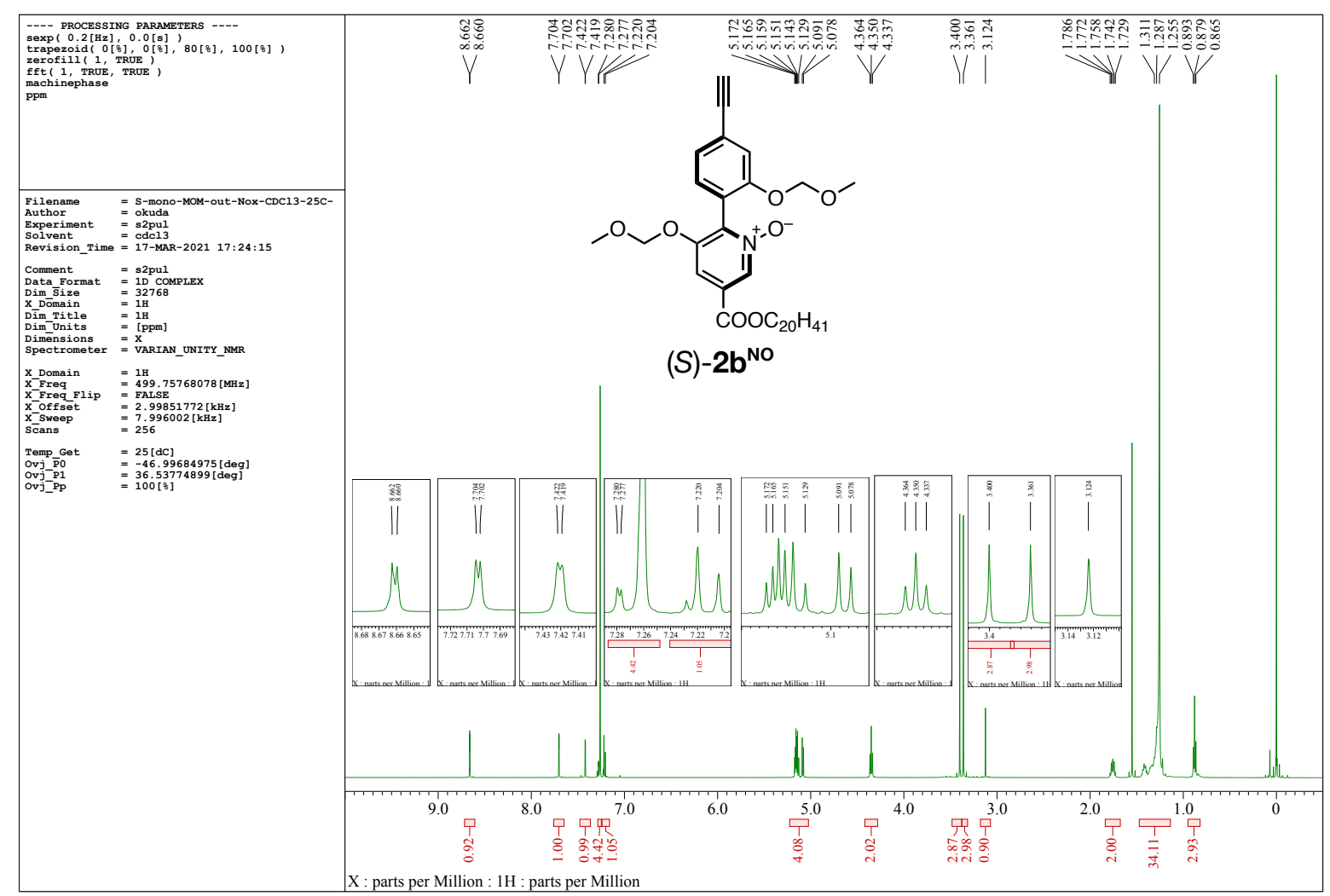

Figure S56. ${ }^{1} \mathrm{H}$ NMR $\left(500 \mathrm{MHz}, \mathrm{CDCl}_{3}, 25^{\circ} \mathrm{C}\right)$ spectrum of $(S)-\mathbf{2} \mathbf{b}^{\mathrm{NO}}$.

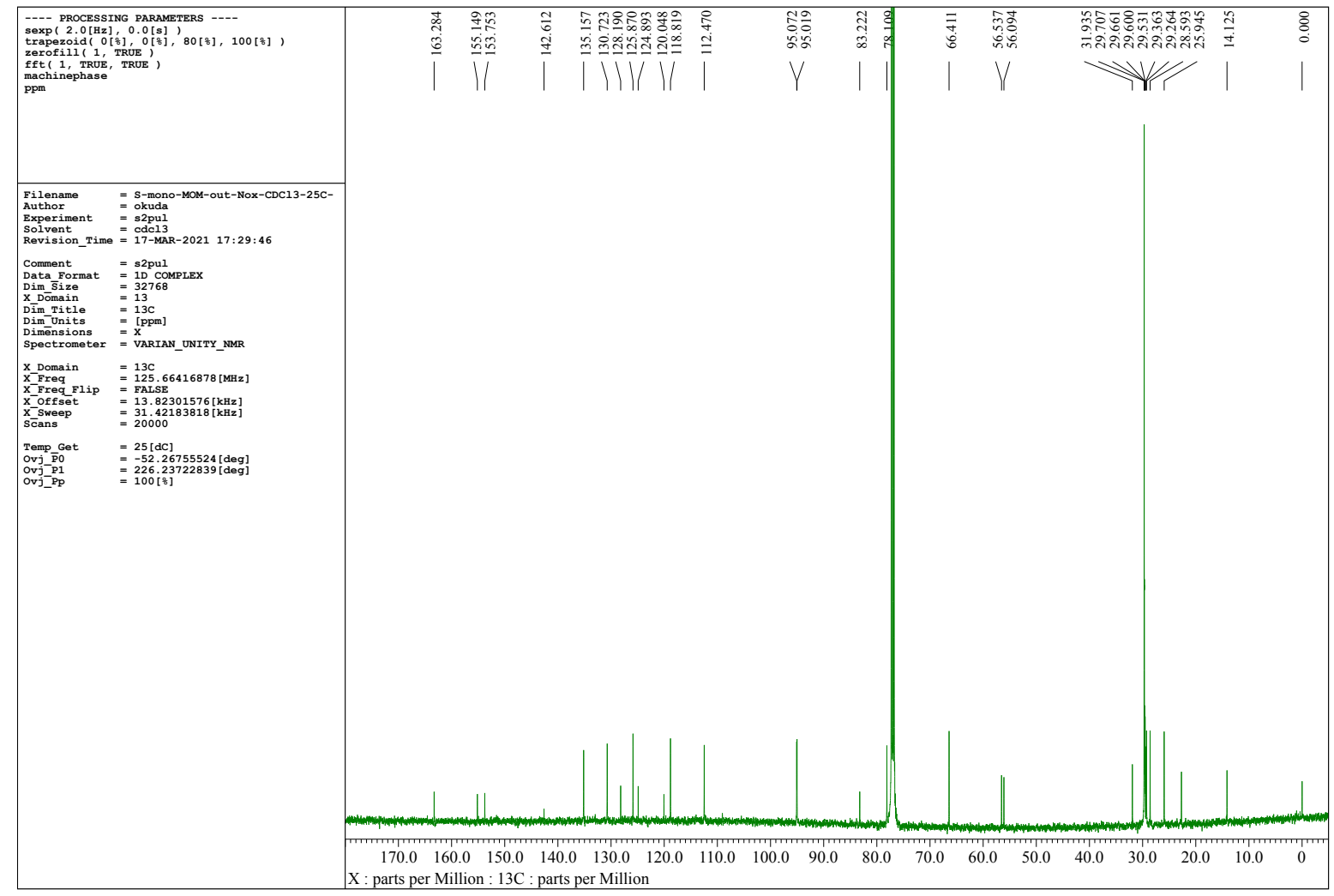

Figure S57. ${ }^{13} \mathrm{C}$ NMR $\left(126 \mathrm{MHz}, \mathrm{CDCl}_{3}, 25{ }^{\circ} \mathrm{C}\right)$ spectrum of $(S)-\mathbf{2} \mathbf{b}^{\text {No }}$. 


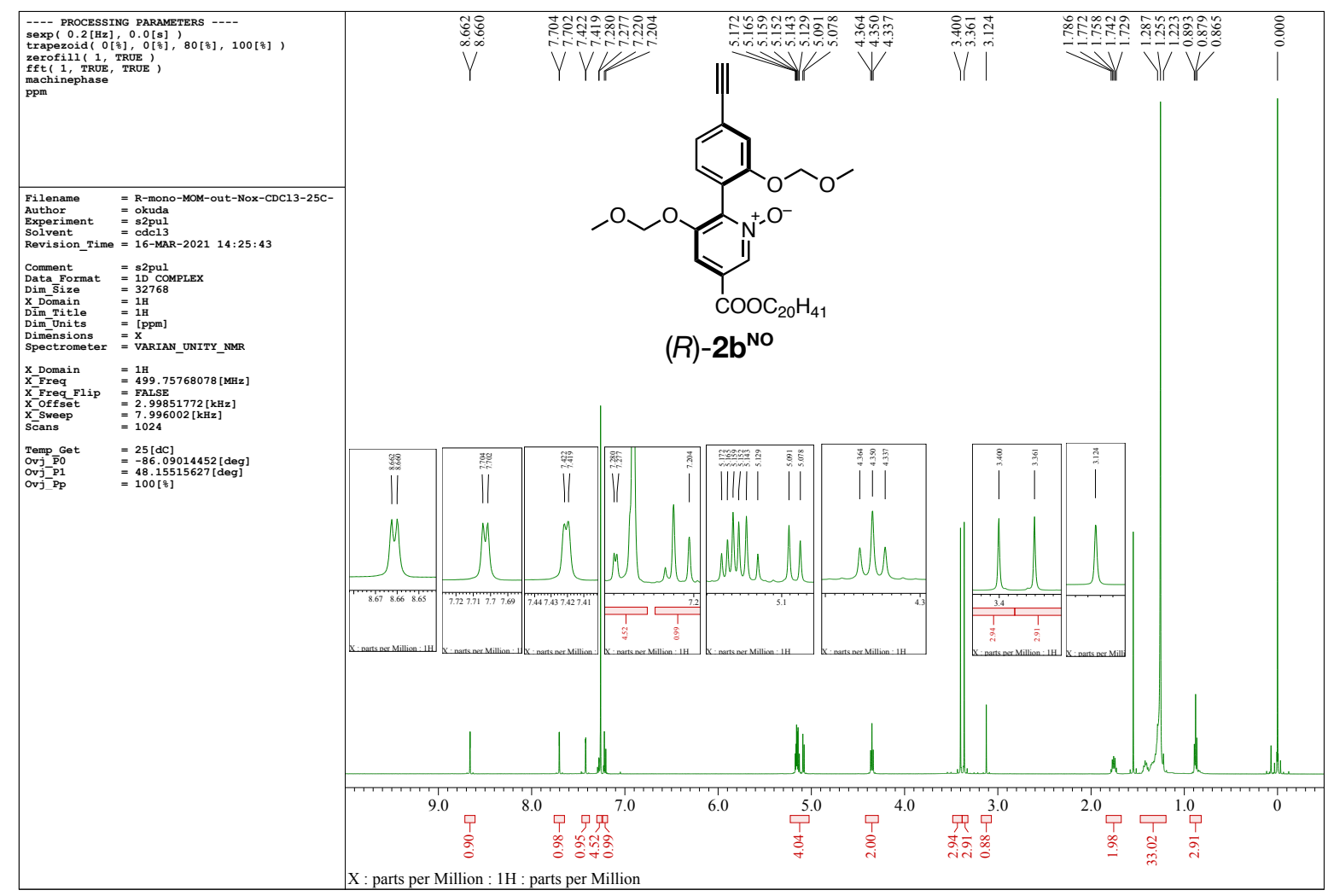

Figure S58. ${ }^{1} \mathrm{H}$ NMR $\left(500 \mathrm{MHz}, \mathrm{CDCl}_{3}, 25^{\circ} \mathrm{C}\right)$ spectrum of $(R)-\mathbf{2} \mathbf{b}^{\text {No }}$.

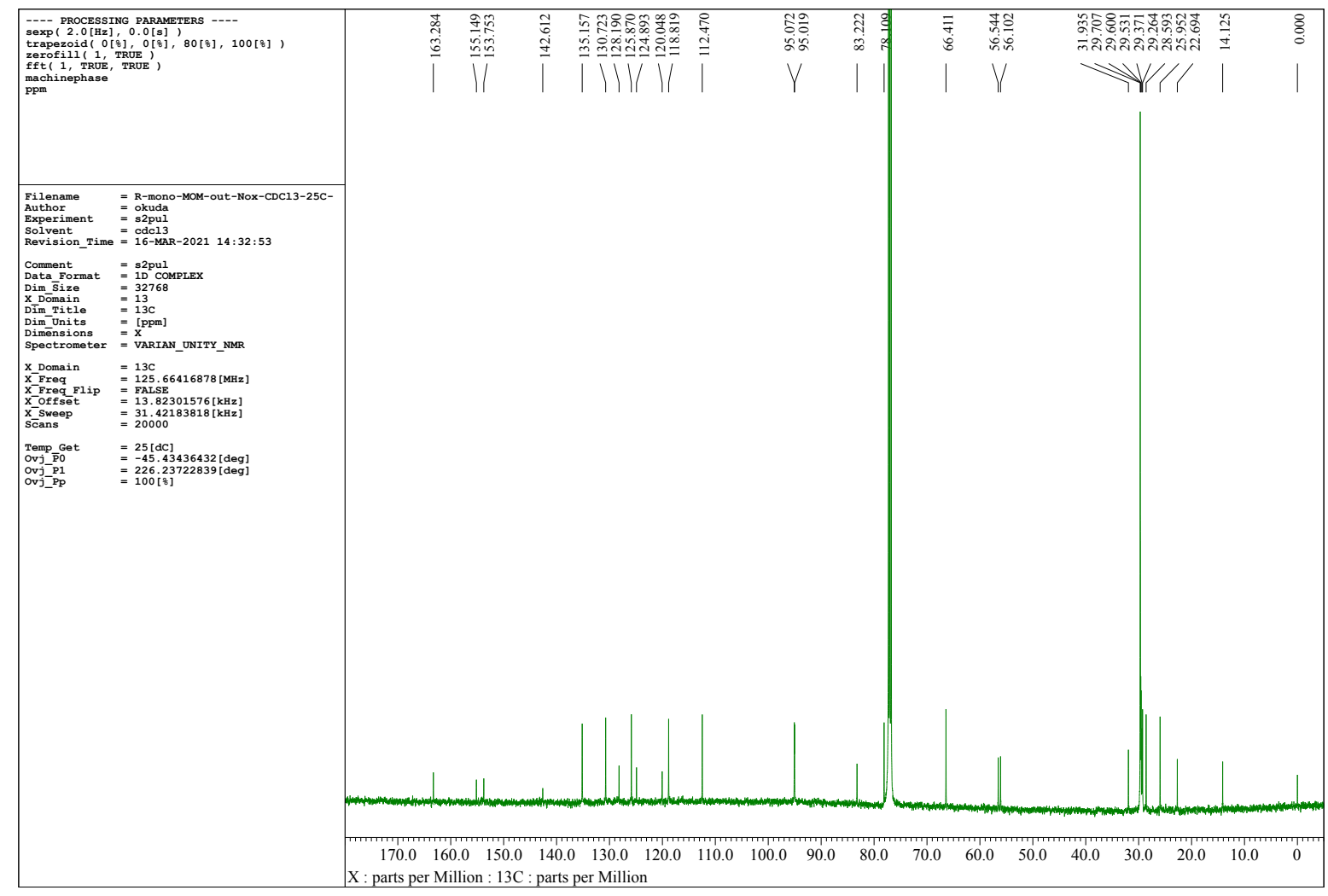

Figure S59. ${ }^{13} \mathrm{C} \mathrm{NMR}\left(126 \mathrm{MHz}, \mathrm{CDCl}_{3}, 25{ }^{\circ} \mathrm{C}\right)$ spectrum of $(R)-\mathbf{2} \mathbf{b}^{\mathrm{NO}}$. 


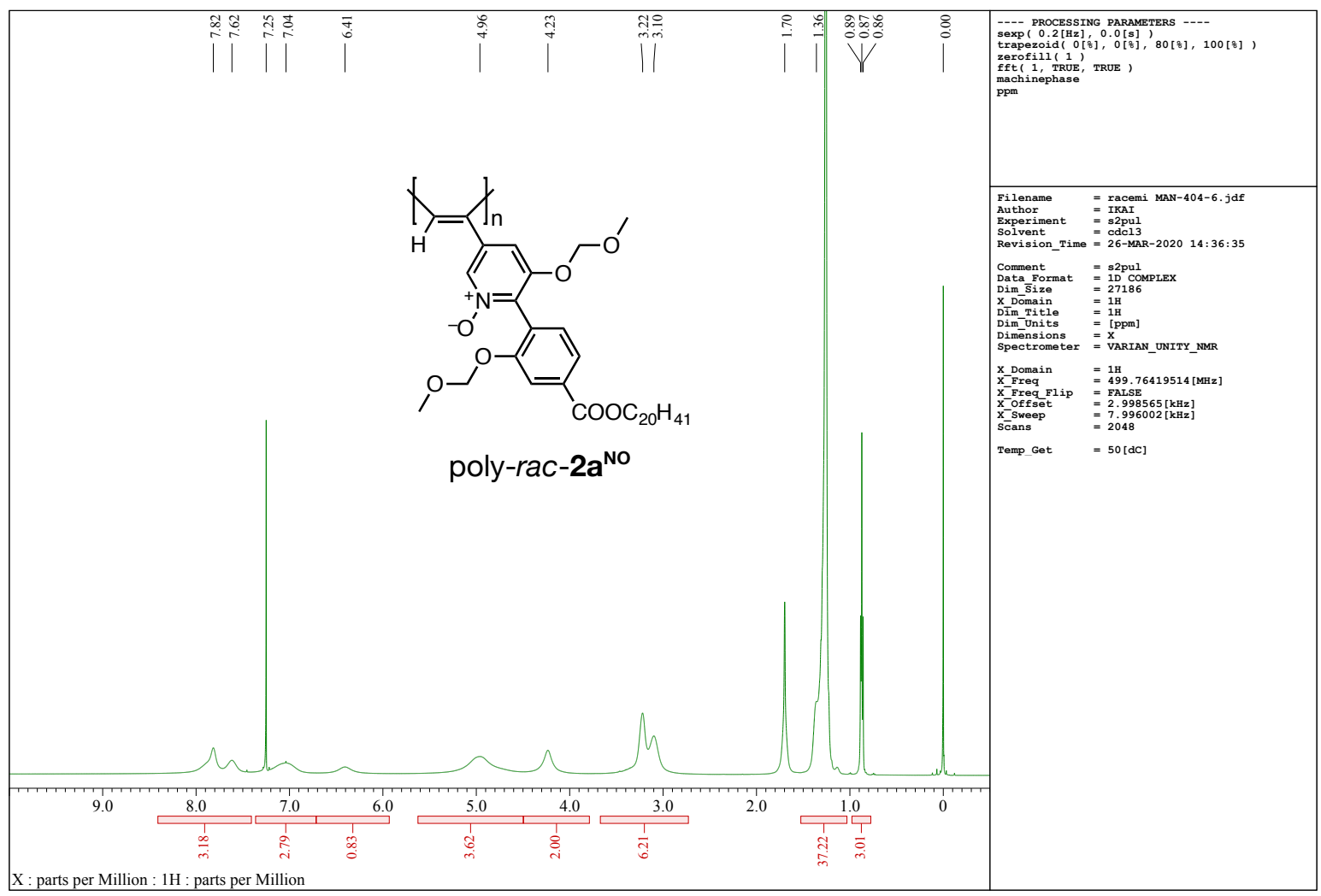

Figure S60. ${ }^{1} \mathrm{H} \mathrm{NMR}\left(500 \mathrm{MHz}, \mathrm{CDCl}_{3}, 50{ }^{\circ} \mathrm{C}\right)$ spectrum of poly-rac-2a ${ }^{\mathrm{NO}}$.

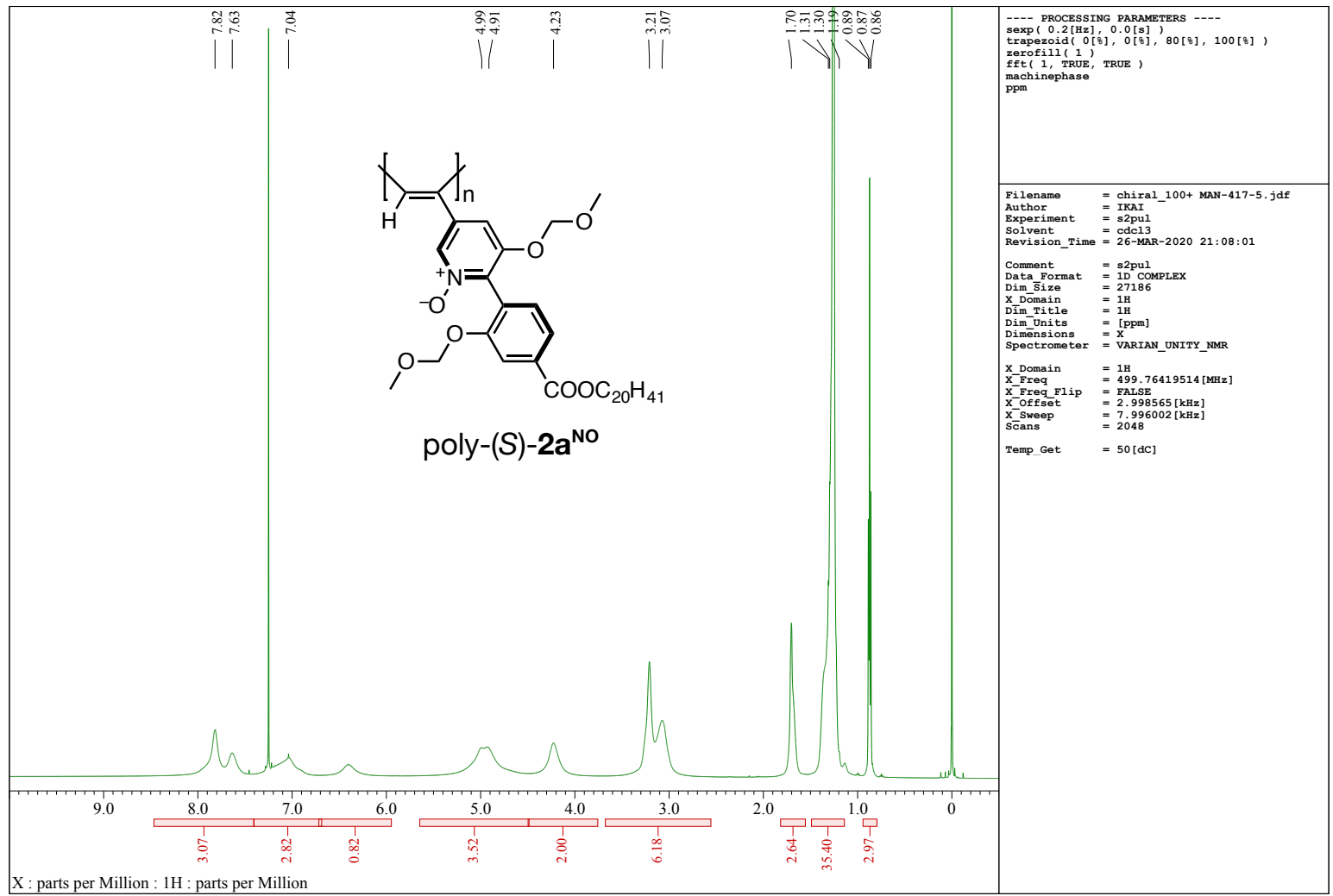

Figure S61. ${ }^{1} \mathrm{H}$ NMR $\left(500 \mathrm{MHz}, \mathrm{CDCl}_{3}, 50{ }^{\circ} \mathrm{C}\right)$ spectrum of poly- $(S)-\mathbf{2 a}{ }^{\mathrm{NO}}$. 


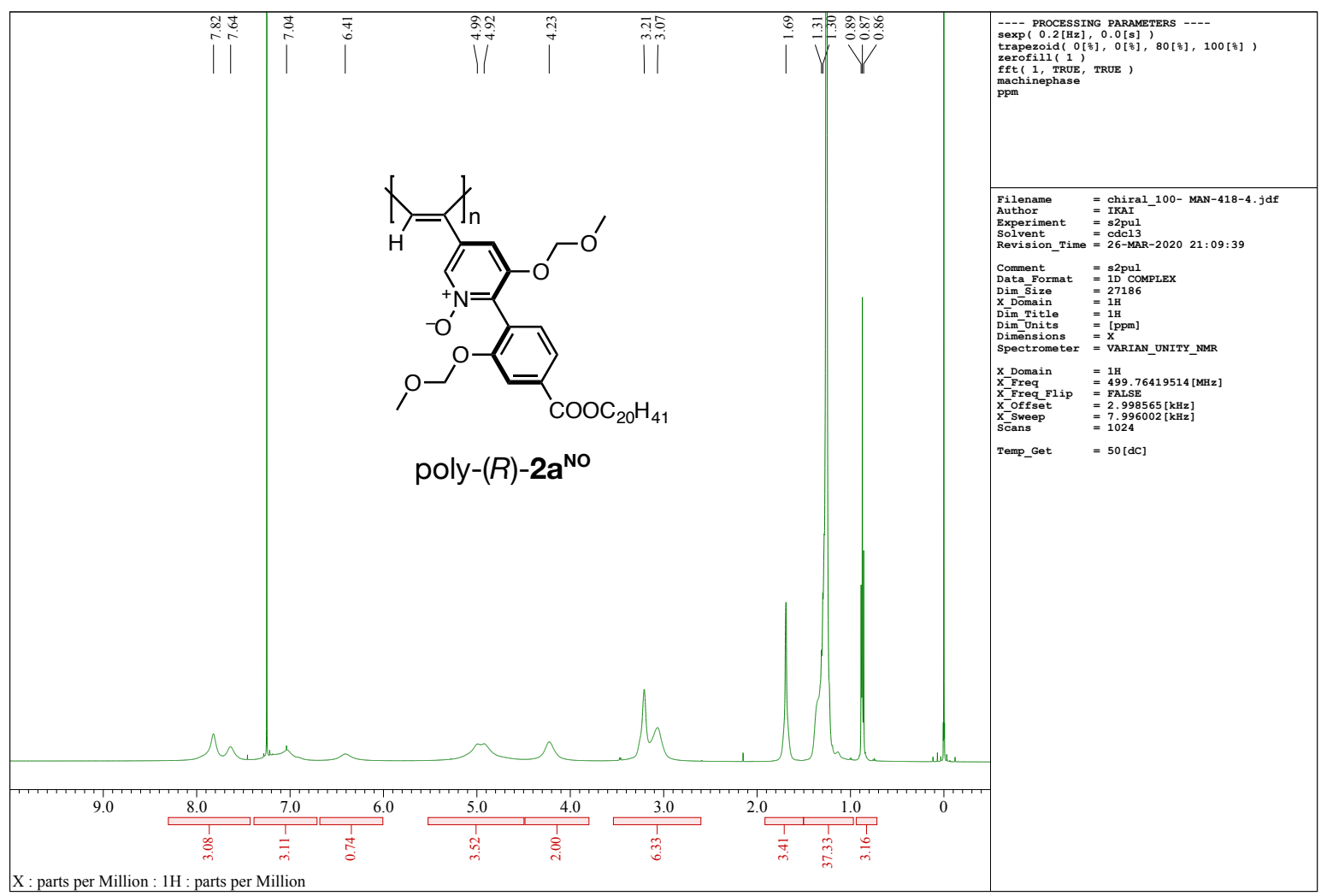

Figure S62. ${ }^{1} \mathrm{H} \mathrm{NMR}\left(500 \mathrm{MHz}, \mathrm{CDCl}_{3}, 50{ }^{\circ} \mathrm{C}\right)$ spectrum of poly- $(R)-\mathbf{2 a}{ }^{\mathrm{NO}}$.

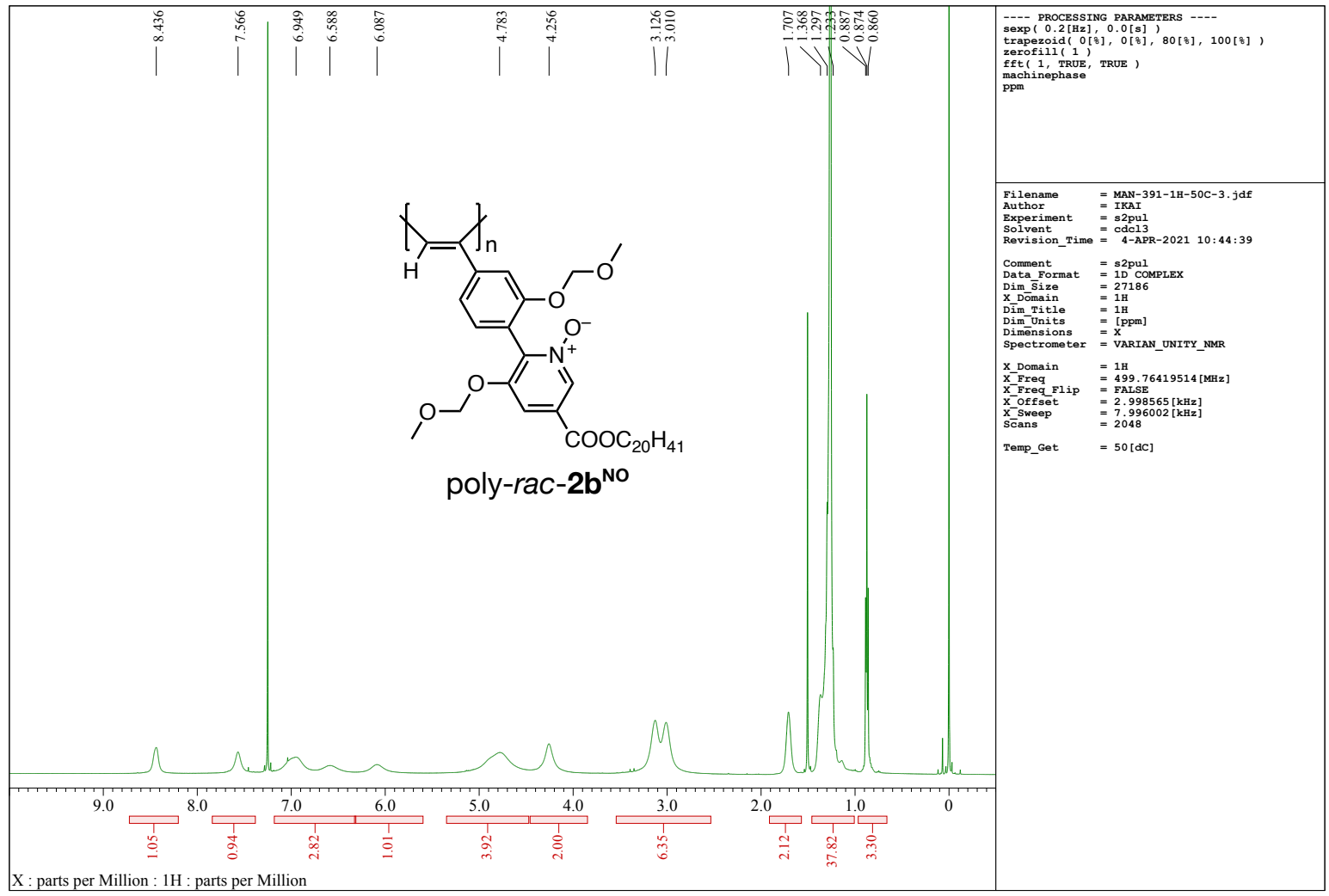

Figure S63. ${ }^{1} \mathrm{H}$ NMR $\left(500 \mathrm{MHz}, \mathrm{CDCl}_{3}, 50{ }^{\circ} \mathrm{C}\right)$ spectrum of poly-rac-2 $\mathbf{b}^{\text {NO }}$. 


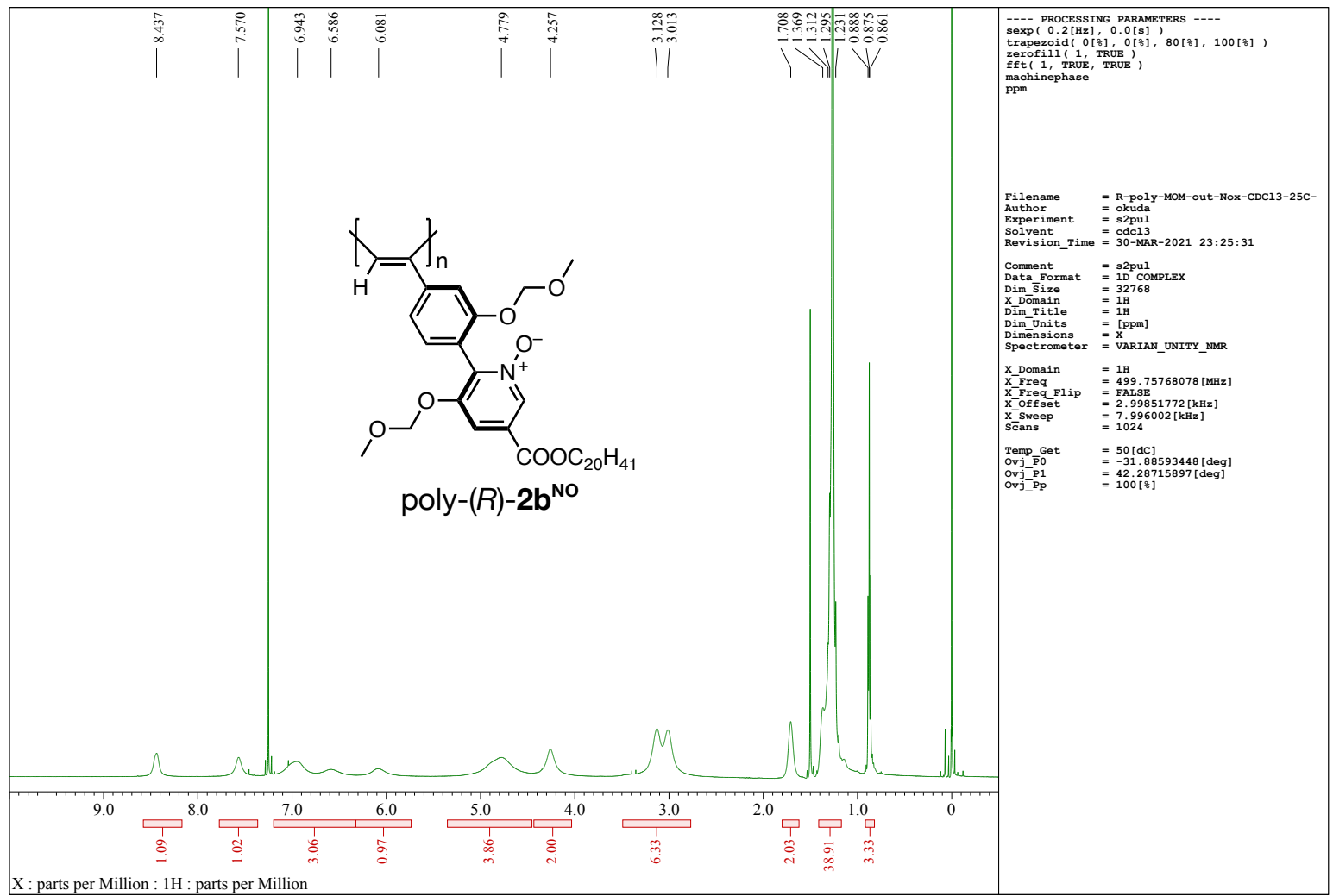

Figure S64. ${ }^{1} \mathrm{H} \mathrm{NMR}\left(500 \mathrm{MHz}, \mathrm{CDCl}_{3}, 50{ }^{\circ} \mathrm{C}\right)$ spectrum of poly- $(R)-\mathbf{2} \mathbf{b}^{\text {NO }}$.

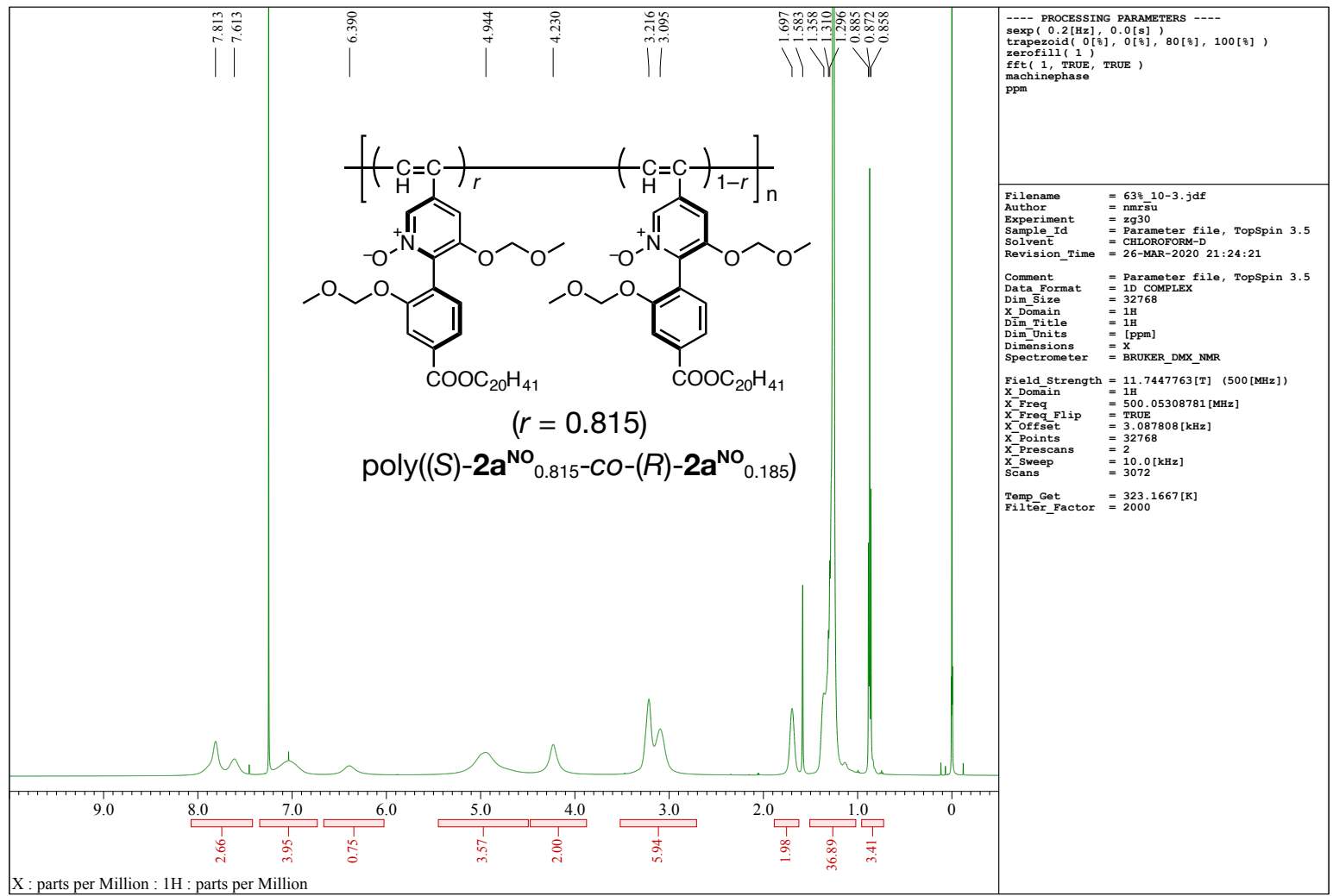

Figure S65. ${ }^{1} \mathrm{H}$ NMR $\left(500 \mathrm{MHz}, \mathrm{CDCl}_{3}, 50{ }^{\circ} \mathrm{C}\right)$ spectrum of poly $\left((S)-\mathbf{2}^{\mathbf{N O}}{ }_{0.815-c o}-(R)-\mathbf{2} \mathbf{a}^{\mathbf{N O}}{ }_{0.185}\right)$. 


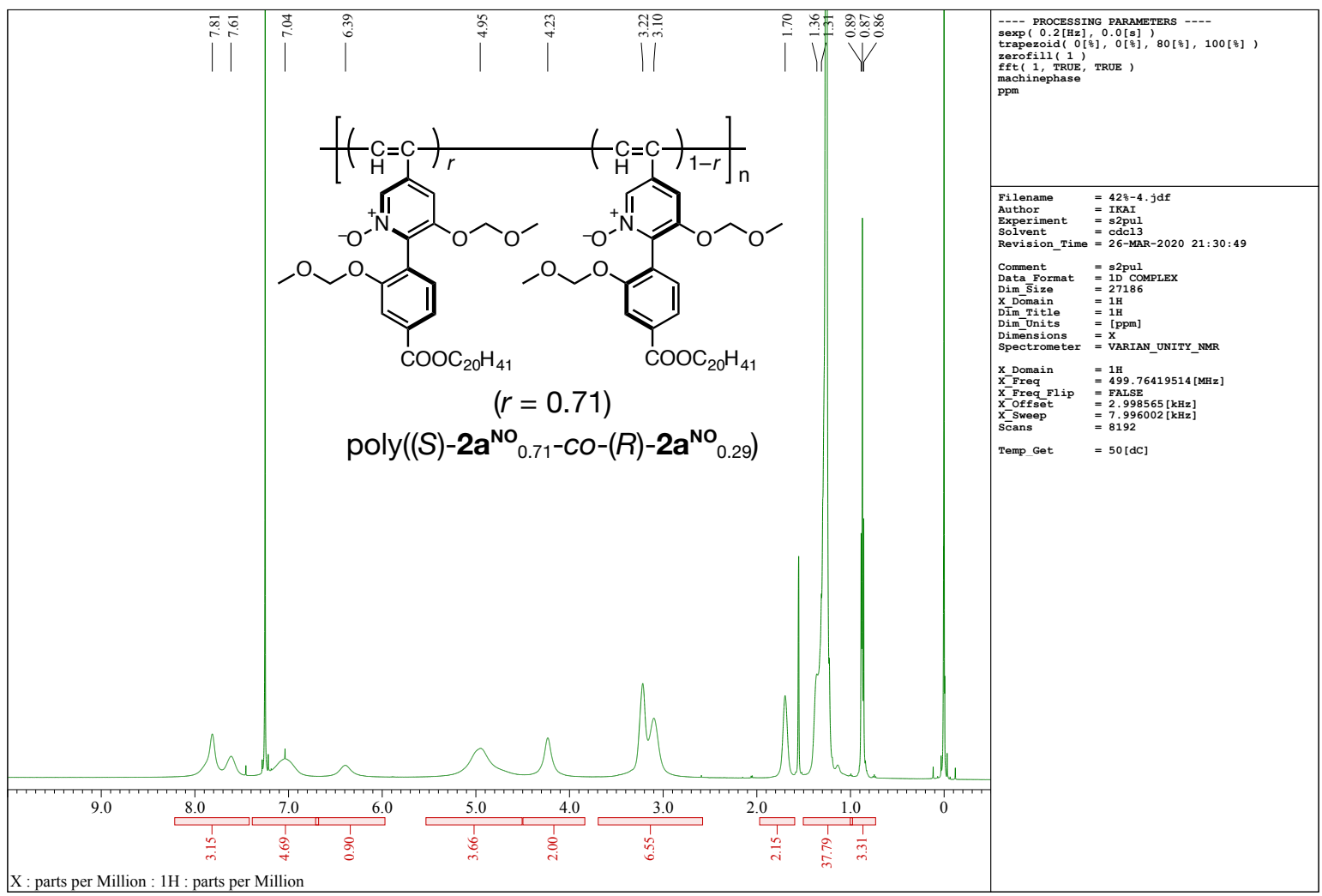

Figure S66. ${ }^{1} \mathrm{H}$ NMR $\left(500 \mathrm{MHz}, \mathrm{CDCl}_{3}, 50{ }^{\circ} \mathrm{C}\right)$ spectrum of poly $\left((S)-\mathbf{2 a}^{\mathbf{N O}}{ }_{0.71}-c o-(R)-\mathbf{2} \mathbf{a}^{\mathbf{N O}}{ }_{0.29}\right)$.

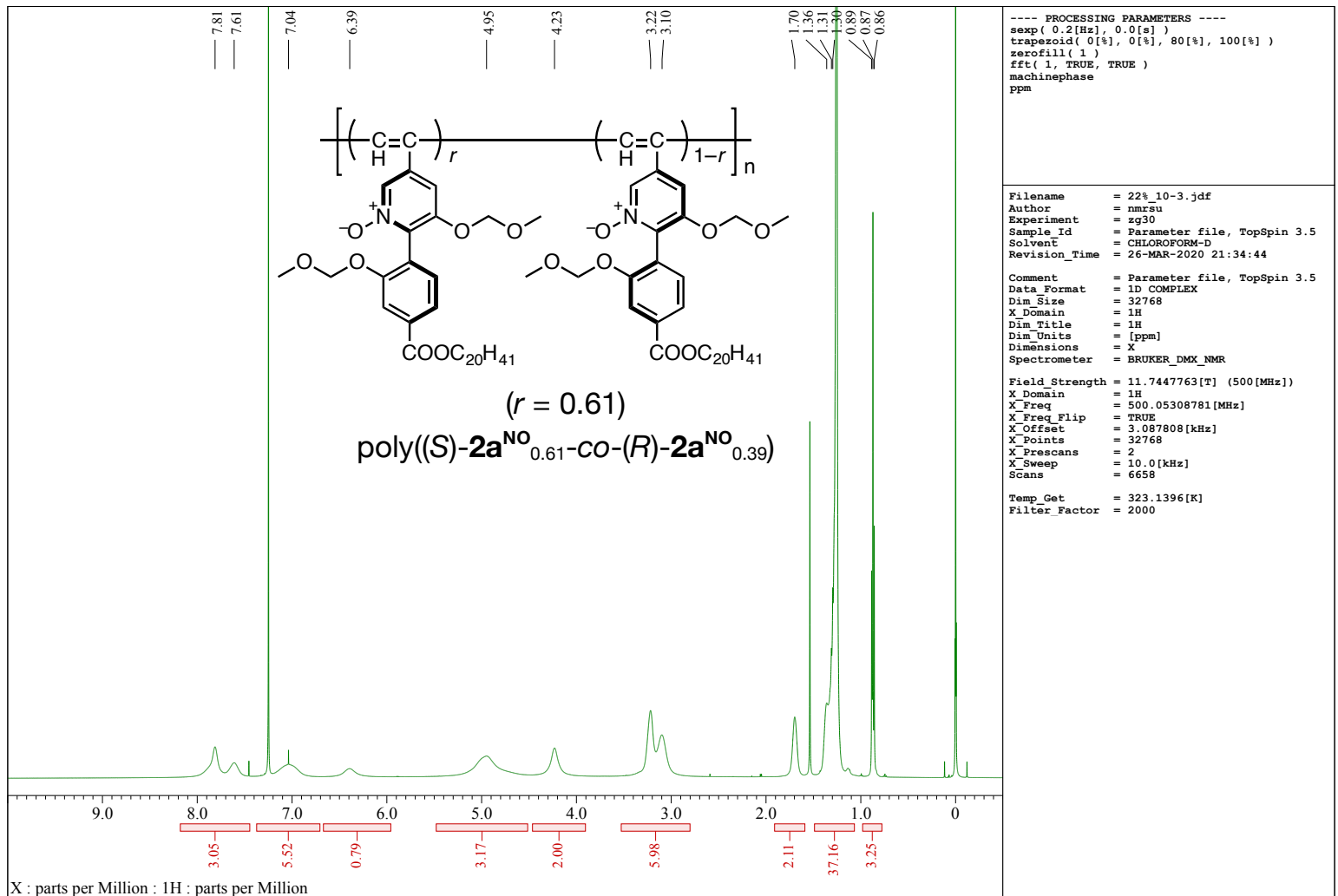

Figure S67. ${ }^{1} \mathrm{H}$ NMR $\left(500 \mathrm{MHz}, \mathrm{CDCl}_{3}, 50{ }^{\circ} \mathrm{C}\right)$ spectrum of poly $\left((S)-\mathbf{2}^{\mathbf{N O}} 0.61-c o-(R)-\mathbf{2} \mathbf{a}^{\mathbf{N O}} 0.39\right)$. 